\title{
The Use of Signal Phase in Dynamic Contrast-Enhanced Magnetic Resonance Imaging
}

by

Claire B. Foottit

\begin{abstract}
A Dissertation submitted to
the Faculty of Graduate Studies and Research

in partial fulfilment of

the requirements for the degree of

Doctor of Philosophy
\end{abstract}

\author{
Department of Physics \\ Carleton University \\ Ottawa, Ontario, Canada
}

September 2010

Copyright (C)

2010 - Claire B. Foottit 


$\begin{array}{ll}\begin{array}{l}\text { Library and Archives } \\ \text { Canada }\end{array} & \begin{array}{l}\text { Bibliothèque et } \\ \text { Archives Canada }\end{array} \\ \begin{array}{l}\text { Published Heritage } \\ \text { Branch }\end{array} & \begin{array}{l}\text { Direction du } \\ \text { Patrimoine de l'édition }\end{array} \\ \begin{array}{l}\text { 395 Wellington Street } \\ \text { Ottawa ON K1A ON4 } \\ \text { Canada }\end{array} & \begin{array}{l}\text { 395, rue Wellington } \\ \text { Ottawa ON K1A ON4 } \\ \text { Canada }\end{array}\end{array}$

Your file Votre référence

ISBN: 978-0-494-70550-6

Our file Notre référence

ISBN: 978-0-494-70550-6

NOTICE:

The author has granted a nonexclusive license allowing Library and Archives Canada to reproduce, publish, archive, preserve, conserve, communicate to the public by telecommunication or on the Internet, loan, distribute and sell theses worldwide, for commercial or noncommercial purposes, in microform, paper, electronic and/or any other formats.

The author retains copyright ownership and moral rights in this thesis. Neither the thesis nor substantial extracts from it may be printed or otherwise reproduced without the author's permission.
AVIS:

L'auteur a accordé une licence non exclusive permettant à la Bibliothèque et Archives Canada de reproduire, publier, archiver, sauvegarder, conserver, transmettre au public par télécommunication ou par l'Internet, prêter, distribuer et vendre des thèses partout dans le monde, à des fins commerciales ou autres, sur support microforme, papier, électronique et/ou autres formats.

L'auteur conserve la propriété du droit d'auteur et des droits moraux qui protège cette thèse. $\mathrm{Ni}$ la thèse ni des extraits substantiels de celle-ci ne doivent être imprimés ou autrement reproduits sans son autorisation.
In compliance with the Canadian Privacy Act some supporting forms may have been removed from this thesis.

While these forms may be included in the document page count, their removal does not represent any loss of content from the thesis.
Conformément à la loi canadienne sur la protection de la vie privée, quelques formulaires secondaires ont été enlevés de cette thèse.

Bien que ces formulaires aient inclus dans la pagination, il n'y aura aucun contenu manquant. 


\section{Abstract}

Tissue perfusion describes nutrient delivery to a capillary bed within a given time frame. An understanding of the perfusion characteristics of a tissue can provide useful physiological information about the state of that tissue, particularly in oncology, where rapidly growing tumours tend to be surrounded by a large number of highly permeable capillaries, which leads to measurable changes in the parameters describing tissue perfusion. These parameters can be measured non-invasively using dynamic contrast-enhanced magnetic resonance imaging (DCE-MRI). This exam involves imaging the patient as a bolus of contrast agent passes through the tissue. To obtain these parameters, information about the concentration of this contrast agent as a function of time in an input artery, called an arterial input function (AIF) is required. Current methods use the magnitude of the MR signal to make this measurement, but these methods are susceptible to significant inaccuracies. The phase of the MR signal can also be used to measure the required input, but, despite several advantages over the MR signal magnitude method, its application has received little attention in the literature.

The purpose of this work was to explore the feasibility of measuring the AIF, using MR signal phase in DCE-MRI exams of tissue perfusion. Initially, issues relating to the implementation of this technique were addressed. Phantom experiments were performed to validate the technique for different clinically relevant set-ups. The geometry of the vessel is an important consideration for accurate measurements using 
phase. Two methods for incorporating the geometry of the vessel were validated through simulation and phantom studies. The phase technique was then validated in two clinical studies; a brain tumour study and a prostate tumour study. The results showed that more reasonable AIFs could be obtained by using the phase method versus the magnitude method.

In conclusion, the use of the MR signal phase for the calculation of the arterial input function in dynamic contrast-enhanced magnetic resonance imaging was shown to be feasible and to provide superior results to conventional approaches. 


\section{Acknowledgments}

Firstly, I would like to thank my supervisor, Dr. Ian Cameron. I really appreciate that he always made himself available whenever I needed advice and took such a keen interest in helping me develop into a well rounded scientist. I have benefited greatly from his open and encouraging research environment, which has allowed me to explore many ideas and ask endless questions. This thesis could not have been what it is without his conscientious editing, constructive criticism and kind encouragement.

I also owe many thanks to all the great members of the MRI lab, from post docs to graduate students to summer students. This positive and collaborative work environment has gone a long way in contributing to the enjoyment and success of my $\mathrm{PhD}$. I would like to specifically acknowledge Dr. Greg Cron, without whom the success of this work would not have been possible. He has provided an endless source of advice, guidance and encouragement. His dedication to medical research and enthusiasm for what we do has been a great inspiration for me.

I have been very fortunate to have had many positive and productive collaborations with several physicians working at the Ottawa Hospital. I would specifically like to acknowledge Dr. Thanh Nguyen, Dr. Leonard Avruch and Dr. Matthew Hogan. Their interest in and commitment to research, as well as our many useful scientific discussions, have made this work possible. I also could not have conducted this research without the support of the technologists in the Department of Diagnostic Imaging at the Ottawa Hospital. Their willingness to accommodate research and 
their interest in improving patient care are an essential component to the success of this work.

I would like to thank several sources that provided funding for my research, including Carleton University, NSERC, Ontario Graduate Scholarships, as well as the generous support of the Kiwanis Club of Ottawa Medical Foundation - Dr. Kanta Marwah scholarship fund. The Ontario Research Development Challenge Fund (ORDCF) - Behavioral Research and Imaging Network (BRAIN) and the Brain Tumour Foundation of Canada also supported some work presented in this thesis.

The support I have received from my friends and family cannot be described in a few short sentences. There are many friends who have, more or less successfully, kept me sane over the last few years. Special thanks go to Amanda, Anita, Brenda, Jessica, Yamile and my sister, Meghan. I would also like to thank my parents who have continually encouraged my scientific spirit since I was a small child. Their love, guidance and support have made many great things in my life possible. Of course we can never repay our parents for all that they have given us, we can only try to pass it on. Finally, a heart felt thank you to my husband whose unconditional love and understanding make any challenge surmountable. He has believed in me every step of the way and has supported me in every way he could (including editing this thesis). I am grateful to share my life with such a wonderful person. 


\section{Table of Contents}

$\begin{array}{ll}\text { Abstract } & \text { ii }\end{array}$

Acknowledgments $\quad$ iv

Table of Contents $\quad$ vi

List of Tables $\quad$ xii

List of Figures $\quad$ xvi

Nomenclature $\quad$ xxiii

1 Introduction $\quad 1$

2 Theory \& Background $\quad 4$

2.1 Tissue Perfusion . . . . . . . . . . . . . . . . . 4

2.1.1 The Vascular System . . . . . . . . . . . . . . 4

2.1.2 Tumour Development and Perfusion Characteristics . . . . . . 7

2.2 Modelling Tissue Perfusion . . . . . . . . . . . . . . . . . 10

2.2.1 Tissue Perfusion Data . . . . . . . . . . . . . 10

2.2.2 General Approach to Perfusion Modelling . . . . . . . . . 11

2.2.3 Modelling Transcapillary Exchange . . . . . . . . . . 15

2.2.4 Two Compartment Models . . . . . . . . . . . . . . 18 
2.2.5 Adiabatic Approximation to the Tissue Homogeneity Model . 21

2.3 MR Signal Generation . . . . . . . . . . . . . . . . . 22

2.3.1 Spins in a Magnetic Field . . . . . . . . . . . . 23

2.3.2 Excitation ......................... 24

2.3.3 Free Precession and Relaxation . . . . . . . . . . 26

2.3.4 The Phase of the Magnetization Vector . . . . . . . . . . . 27

2.4 The MRI Image . . . . . . . . . . . . . . . . . . . . . . . 29

2.4.1 Signal Detection ................... 29

2.4 .2 Localization . . . . . . . . . . . . . . 30

2.4.3 Image Formation . . . . . . . . . . . . . . . 34

2.5 Noise Properties of the MRI Signal . . . . . . . . . . . . . . 35

2.5.1 Noise in Magnitude Images . . . . . . . . . . . . . 36

2.5.2 Noise in Phase Images . . . . . . . . . . . . . . . . 37

2.6 The Spoiled Gradient Echo Sequence . . . . . . . . . . . . . . . 38

2.6.1 Signal Magnitude . . . . . . . . . . . . . . 40

2.6.2 Signal Phase. . . . . . . . . . . . . . . . 42

2.7 Materials in a Magnetic Field . . . . . . . . . . . . . . 42

2.7.1 Magnetic Susceptibility . . . . . . . . . . . . 43

2.7.2 Analytic Solution for a Cylinder . . . . . . . . . . . . 44

2.7.3 Dipole Approximation Method . . . . . . . . . . . . 48

2.8 Method for Dynamic Contrast-Enhanced MRI . . . . . . . . . . . . 49

2.8.1 The MRI Contrast Agent and its Effect on the NMR Tissue Properties ........................ 50

2.8.2 The Effect of the Contrast Agent on Signal Phase . . . . . . . 52

2.8.3 Effect of Contrast Agent on the Spoiled Gradient Echo Sequence 55

2.8.4 $T_{1}$ Map Using the Variable Flip Angle Method . . . . . . . . . 59 
2.8.5 Using the Magnitude of the MRI Signal to Measure Contrast Agent Concentration . . . . . . . . . . . . . 62

3 Motivation and Proposed Research $\quad 68$

3.1 Literature Review . . . . . . . . . . . . . . . 72

3.2 Outline of Proposed Research . . . . . . . . . . . . . . 74

4 Phantom Experiments $\quad 79$

4.1 Introduction . . . . . . . . . . . . . . . 79

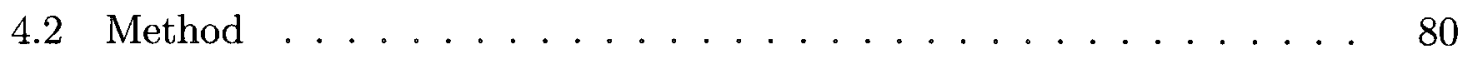

4.2.1 Blood Vessel Phantom . . . . . . . ...... 80

4.2.2 Phase Data Acquisition . . . . . . . . . . . . 81

4.2.3 Phase Analysis Method . . . . . . . . . . . . 82

4.2.4 $\mathrm{T}_{1}$ Measurement .................... 85

4.3 Results ......................... 86

4.3.1 Straight-Tube Phantoms . . . . . . . . . . . 87

4.3.2 Curved-Tube Phantoms . . . . . . . . . . . . . . 92

4.3.3 Thin Walled Phantom ............... 93

$4.3 .4 \mathrm{~T}_{1}$ Results....................... 95

4.4 Discussion . . . . . . . . . . . . . . . . . . 98

4.4.1 Geometry Factor, $g(\theta)$, for the Straight Tube Phantom . . . . 98

4.4.2 Geometry Factor, $g(\theta)$, for the Curved Tube Phantom . . . . . 99

4.4.3 Echo Time................... 100

4.4 .4 Field of View . . . . . . . . . . . . . . 101

4.4.5 Over and Underestimation .............. 103

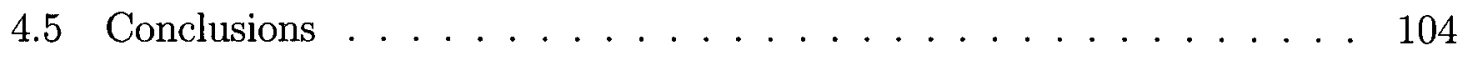

5 Calculating the Geometry Factor 105 


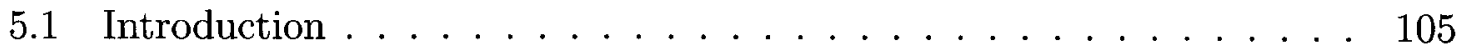

5.2 Method . . . . . . . . . . . . . . . . 106

5.2.1 Dipole Approximation Method . . . . . . . . . . . . . 107

5.2 .2 Simulated Blood Vessels . . . . . . . . . . . . . . . . . 109

5.2 .3 Phantom Tubes . . . . . . . . . . . . . . . . . . 111

5.2 .4 Clinical Cases . . . . . . . . . . . . . . . . . . 112

5.3 Results . . . . . . . . . . . . . . . . . . . . . . 114

5.3.1 Validation of the Dipole Approximation Method on an Infinite Cylinder . . . . . . . . . . . . . . . . . . . 114

5.3 .2 Phantom Validation . . . . . . . . . . . . 124

5.3.3 Straight Tube Approximation . . . . . . . . . . . . 128

5.3.4 Method Applied to Clinical Data . . . . . . . . . . . . 134

5.4 Discussion . . . . . . . . . . . . . . . . 136

5.4.1 Assess Method on Known Geometry . . . . . . . . . . 136

5.4 .2 Phantom Validation $\ldots \ldots \ldots \ldots \ldots$

5.4.3 Straight Tube Approximation . . . . . . . . . . . . 140

5.4 .4 Clinical Cases . . . . . . . . . . . . . . . . . . . 141

5.5 Conclusions . . . . . . . . . . . . . . . . . . 142

6 Brain Tumour Study 144

6.1 Introduction . . . . . . . . . . . . . . . . . . . 144

6.2 Methods . . . . . . . . . . . . . . . . . . . . . . 146

6.2.1 The Clinical Protocol . . . . . . . . . . . . . . . . . . 146

6.2 .2 Signal Phase Extraction $\ldots \ldots \ldots \ldots$

6.2.3 Determination of Input/Output Functions . . . . . . . . . 149

6.2.4 Determination of Tissue Concentration . . . . . . . . 152

6.2.5 The Perfusion Map Method . . . . . . . . . . . . . 152 
6.3 Results . . . . . . . . . . . . . . . . . . . . 153

6.3.1 Input and Output Functions . . . . . . . . . . . . . 154

6.3 .2 Perfusion Maps . . . . . . . . . . . . . . . . 160

6.4 Discussion . . . . . . . . . . . . . . . . . 167

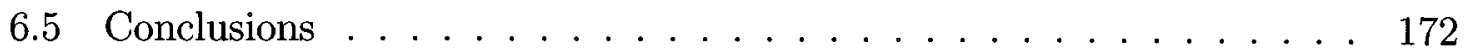

7 Prostate Tumour Study 174

7.1 Introduction . . . . . . . . . . . . . . . . . . . . . 174

7.2 Methods . . . . . . . . . . . . . . . . . . 176

7.2 .1 The clinical protocol $\ldots \ldots \ldots \ldots \ldots$

7.2 .2 The AIF Selection Method . . . . . . . . . . . . . . 177

7.2 .3 The Obturator Internus Muscle . . . . . . . . . . . . 180

7.2 .4 The Prostate . . . . . . . . . . . . . . . . 180

7.3 Results . . . . . . . . . . . . . . . . . . . . . . . . . 182

7.3.1 AIF Method Comparison . . . . . . . . . . . . . . . . 182

7.3.2 Perfusion in the Obturator Internus Muscle . . . . . . . . 182

7.3 .3 Perfusion in Prostate . . . . . . . . . . . . . . . . . 187

7.4 Discussion . . . . . . . . . . . . . . . . . . . . 191

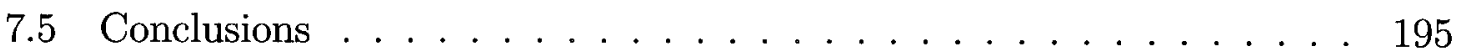

$\begin{array}{lll}8 \text { Conclusion } & 197\end{array}$

8.1 Overview of Problem . . . . . . . . . . . . . . . . . 197

8.2 Implementation . . . . . . . . . . . . . . . . . . . . 199

8.2 .1 Phantom Studies . . . . . . . . . . . . . 200

8.2 .2 Geometry Factor . . . . . . . . . . . . . . 201

8.3 Clinical Implementation . . . . . . . . . . . . . . . . . . 202

8.3.1 Brain Tumour Study . . . . . . . . . . . . . . 203

8.3 .2 Prostate Tumour Study . . . . . . . . . . . . . 204 
8.4 Summary . . . . . . . . . . . . . . . . . . 205

$\begin{array}{ll}\text { List of References } & 207\end{array}$

Appendix A Multiple Coil Combination $\quad 215$

Appendix B Code for Marques and Bowtell method 219

B.1 Main Body of the Code . . . . . . . . . . . . . . . . . . 219

B.2 The Function $B d r \ldots \ldots \ldots \ldots \ldots \ldots \ldots \ldots \ldots \ldots \ldots$ 


\section{List of Tables}

2.1 Classification of materials based on magnetic susceptibility. . . . . . . 44

4.1 Summary of experimental parameters. The spatial resolution (SR) was calculated from the non-zerofilled matrix size. . . . . . . . . . . . 83

4.2 Theoretical relationship between $[\mathrm{CA}]$, concentration of contrast agent (mM) and $\phi$ (radians) for the $T_{E}(\mathrm{~ms})$ used in the blood vessel phantom study. ....................... 86

4.3 Summary of 2 factor ANOVA results. . . . . . . . . . . . . . 91

4.4 Summary of [CA] (mM) predicted by phantom straight tube experiments, $\left[\mathrm{CA}_{1 m M}\right]$, at various $T_{E}$ 's and FOVs if $1 \mathrm{mM}$ of $\mathrm{CA}$ is present. $\quad 91$

4.5 Calculated $T_{1}$ results for the blood vessel phantom using the inversion recovery and variable flip angle methods. An assumed $T_{1}$ for water of $4000 \mathrm{~ms}$ was used to convert from [CA] to the expected values of $T_{1}$ for Gadovist $\mathbb{R}$ given in column 2. . . . . . . . . . . . . . . . 97

5.1 Polynomial fits used to generate simulated vessels. . . . . . . . . . 110

5.2 Simulation results for $B_{d}(\mathbf{r})$ and $C$ for an infinitely long cylinder at various angles. The cylinder was filled with a $1 \mathrm{mM}$ solution of CA and was surrounded by water. . . . . . . . . . . . . . 117

5.3 Simulation results for $B_{d}(\mathbf{r})$ and $C$ for infinitely long cylinders of various radii for $\theta=0^{\circ}$ and $45^{\circ}$ with respect to $B_{0} \hat{z}$. The cylinder was filled with a $1 \mathrm{mM}$ solution of $\mathrm{CA}$ and was surrounded by water. . . . 118 
5.4 Simulation results for $B_{d}(\mathbf{r})$ and $C$ for an infinitely long cylinder of radius 16 pixels parallel to $B_{0} \hat{z}$, with the addition of a second parallel cylinder of varying radius. The cylinders were both filled with $1 \mathrm{mM}$ solution of CA and was surrounded by water. . . . . . . . .

5.5 Simulation results for $B_{d}(\mathbf{r})$ and $C$ for an infinitely long cylinder of radius 16 pixels at various $[\mathrm{CA}] \mathrm{s} . \ldots \ldots \ldots \ldots$

5.6 Simulation results for $B_{d}(\mathbf{r})$ and $C$ for an infinitely long cylinder of radius 16 pixels with smoothing. The cylinder was filled with a $1 \mathrm{mM}$ solution of CA and was surrounded by water. The term "nna $3 \times 2$ " indicates that the smoothing was applied twice. . . . . . . . . 123

5.7 Statistics for $\Delta \phi_{\text {exp }} / \Delta \phi_{s i m}$ under different inclusion criteria. . . . . 127

5.8 Simulation results for $B_{d}(\mathbf{r})$ and $C$ for cylinders of radius 16 pixels and various lengths. . . . . . . . . . . . . .

5.9 Simulation results for $B_{d}(\mathbf{r})$ and $C$ for a torus with radius of curvature equal to 128 pixels and a radius of 16 pixels. The $B_{d}(\mathbf{r})$ values were compared to $B_{d}(\mathbf{r})_{s t}$, the field expected for an infinitely long straight cylinder oriented parallel to the tangent to the torus at the given location.132

5.10 Comparison between $B_{d}(\mathbf{r})$ calculated using a simulated polynomial and using the straight tube approximation. . . . . . . . . . 135

5.11 A comparison of the geometry factor calculated using the straight vessel approximation, $g(\mathbf{r})_{s t}$, and a segmented vessel from the SSS, $g(\mathbf{r})_{c l}$.

6.1 Summary of average AIF and VOF characterization parameters. The results are presented as mean (SD). There were twenty-eight studies included in each mean except for $\mathrm{AIF}_{\phi}$ where twenty-five cases were used, three cases were excluded because the neck coil was not used and thus there was not sufficient SNR in the neck slice to analyze this data. 155 
6.2 $K^{\text {trans }}\left(\mathrm{min}^{-1}\right)$ values obtained from tracer kinetic modelling using the NordicIce software package where the AIF used in the analysis was $\mathrm{AIF}_{\phi}, \mathrm{VOF}_{\phi}$ (time shifted) or $\mathrm{VOF}_{|S|}$ (time shifted). Note: entries marked $0^{*}$ indicate that NordicIce was not able to perform the fitting due to insufficient tumour enhancement. . . . . . . . . . . . . 162

$6.3 v_{p}$ values obtained from tracer kinetic modelling using the NordicIce software package where the AIF used in the analysis was $\mathrm{AIF}_{\phi}, \mathrm{VOF}_{\phi}$ (time shifted) or $\mathrm{VOF}_{|S|}$ (time shifted). Note: entries marked $0^{*}$ indicate that NordicIce was not able to perform the fitting due to insufficient tumour enhancement. . . . . . . . . . . . . . . . . . .

6.4 $v_{e}$ values obtained from tracer kinetic modelling using the NordicIce software package where the AIF used in the analysis was $\mathrm{AIF}_{\phi}, \mathrm{VOF}_{\phi}$ (time shifted) or $\mathrm{VOF}_{|S|}$ (time shifted). Note: entries marked $0^{*}$ indicate that NordicIce was not able to perform the fitting due to insufficient tumour enhancement. . . . . . . . . . . . . . . 165

6.5 Linear fit between perfusion parameters derived using the indicated inputs. Eight studies were included in the comparison. . . . . . . . 165

6.6 Perfusion parameters in grade IV gliomas. Values show inter-patient mean and SD for the current study for grade IV gliomas using $\mathrm{VOF}_{|S|}$, $\mathrm{VOF}_{\phi}$ and $\mathrm{AIF}_{\phi}$. Values from the literature are also provided for com-

7.1 A description of the slice grouping. The slices included in each slice block and the location of the center of the block relative to the inferior edge of the complete $3 \mathrm{D}$ slice block are given. . . . . . . . . . . 
7.2 Comparison of perfusion parameters measured in the OIM using four different AIFs, as well as results from the literature. All data is for human OIM except for the study by Yankeelov et al. which was performed in rats. . . . . . . . . . . . . . . . . . . . . 186

7.3 Perfusion parameters in the prostate. Values show inter-patient mean and SD for the current study using $|S|$ - and $\phi$-derived AIFs and for studies from the literature. ${ }^{*} \mathrm{CT}$ perfusion does not use the same CA. 190

7.4 Maximum of mean tissue $[\mathrm{CA}]$ in $\mathrm{mmol} / \mathrm{kg}$ per $0.1 \mathrm{mmol} / \mathrm{kg}$ injection dose to facilitate comparison with the literature. Values show interpatient mean and SD for the current study using the $|S|$-derived TRF and for studies from the literature. Note a tissue density of $1 \mathrm{~g} / \mathrm{mL}$ is assumed. . . . . . . . . . . . . . . . . . . . . 191 


\section{List of Figures}

2.1 The vascular system transports nutrients and oxygen to the tissue and removes waste and carbon dioxide from the tissue. . . . . . 8

2.2 A schematic of information extracted from a dynamic contrastenhanced (DCE-MRI) exam. . . . . . . . . . . . . . . 11

2.3 Simplified tissue model . . . . . . . . . . . . . . . . . . 15

2.4 View of the magnetization after an RF pulse with flip angle, $\alpha$, in a reference frame rotating at $\omega_{r f}=\omega_{0} \ldots \ldots \ldots \ldots 26$

2.5 $M_{z}$ and $M_{x y}$ relaxation after an RF excitation. See also Eqs. 2.46 and

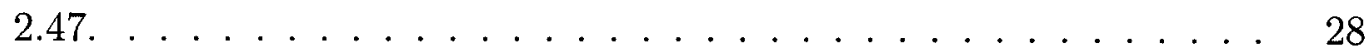

2.6 Schematic of gradient echo formation. . . . . . . . . . . . . 32

2.7 Schematic of the effect of $|S|$ on $\sigma_{\phi}$. The arrow indicates $A$ the true MR signal without noise and the error bars represent $\sigma_{g}$ the error in $S_{R}$ and $S_{I}$. From this one can imagine a distribution of signal vectors, $\mathbf{S}$, represented by the shaded gray area. By comparing the two diagrams it can be seen that the diagram on the right with the longer vector $\mathbf{A}$ has less of a range of angles and thus $\sigma_{\phi}$ is lower. . . . . . . 38

2.8 A sequence diagram for a 2D spoiled gradient echo (SPGR) sequence. 39

2.9 Coordinate system definition for cylinder in a magnetic field. . . . . . 45 
2.10 Phase difference for a infinite straight vessel at an angle $\theta$ with respect to $\mathbf{B}_{\mathbf{0}}$ divided by the phase difference at $\theta=0^{\circ}$, for the same change

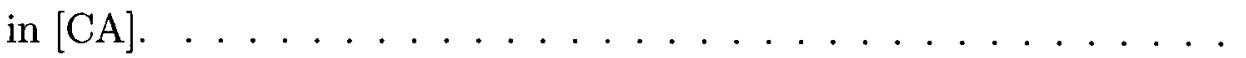

2.11 Graph showing the non-linear relationship between signal magnitude from an SPGR sequence (A.U.) and [CA] for a typical 3D protocol and several pre-contrast $T_{1}$ values typical of tissue. The curves for $T_{1,0}=1500 \mathrm{~ms}, T_{1,0}=1000 \mathrm{~ms}$ and $T_{1,0}=500 \mathrm{~ms}$ shown as solid, dotted and dashed lines, respectively. . . . . . . . . . . . .

2.12 Graph showing the relationship between the complex signal from an SPGR sequence and $[\mathrm{CA}] \ldots \ldots \ldots \ldots \ldots \ldots$

2.13 Diagram showing an example object with a pixel grid to illustrate the concept of partial volumes and signal leakage. . . . . . . . . .

2.14 Phase signal corruption leading to an overestimation of [CA]. a) A typical input function. b) The complex MR signal with and without corruption from an external signal. c) The measured phase accumulation with and without signal corruption. d) The measured [CA] with and without signal corruption. Note that the points in b) evolve as a function of time from the points labelled "baseline" to the points labelled "peak" and then back towards the baseline again. . . . . . 60

2.15 Phase signal corruption leading to an underestimation of [CA]. a) A typical input function. b) The complex MR signal with and without corruption from an external signal. c) The measured phase accumulation with and without signal corruption. d) The measured [CA] with and without signal corruption. Note that the points in b) evolve as a function of time from the points labelled "baseline" to the points labelled "peak" and then back towards the baseline again. . . . . . 
2.16 Schematic of a DCE-MRI acquisition with a pre-contrast $T_{1}$ map acquired using a variable flip angle (VFA) approach. . . . . . . . . .

2.17 Schematic of a DCE-MRI acquisition with bookend $T_{1}$ maps acquired using a variable flip angle (VFA) approach. . . . . . . . . . . 65

4.1 Schematic showing phantom experiments for straight and curved tubes. The i.d. is inner diameter and o.d. is outer diameter. . . . . . . . . 82

$4.2 \phi$ as a function of $[\mathrm{CA}]$ for a straight tube parallel to $B_{0} \hat{z}$ with $T_{E}=$ $5.48 \mathrm{~ms}$ for several FOVs. Error bars represent 1 SD of 9 image averages. 88

$4.3 \phi$ as a function of [CA] for a straight tube parallel to $B_{0} \hat{z}$ with $T_{E}=$ $2.06 \mathrm{~ms}$ for several FOVs. Error bars represent $1 \mathrm{SD}$ of 9 image averages. 88

$4.4\left(\lambda_{\phi}\right)_{\text {exp }} /\left(\lambda_{\phi}\right)_{\text {theory }}$ as a function of angle, $\theta$, for several FOVs for a straight tube parallel to $B_{0} \hat{z}$, with $T_{E}=5.48 \mathrm{~ms}$. The thick solid line shows the theoretical calculation from Eq. 2.113. The error bars represent the standard error calculated from the linear regression. . .

$4.5\left(\lambda_{\phi}\right)_{\text {exp }} /\left(\lambda_{\phi}\right)_{\text {theory }}$ as a function of angle, $\theta$, for several FOVs for a straight tube parallel to $B_{0} \hat{z}$, with $T_{E}=2.06 \mathrm{~ms}$. The thick solid line shows the theoretical calculation from Eq. 2.113. The error bars represent the standard error calculated from the linear regression. . .

$4.6\left(\lambda_{\phi}\right)_{\text {exp }} /\left(\lambda_{\phi}\right)_{\text {theory }}$ as a function of angle, $\theta_{t}$, for several FOVs for a curved tube $\left(T_{E}=5.48 \mathrm{~ms}\right)$. The thick solid line shows the theoretical value calculated for Eq. 2.113. The error bars represent the standard error calculated from the linear regression. . . . . . . . . . . . . 94

$4.7\left(\lambda_{\phi}\right)_{\text {exp }} /\left(\lambda_{\phi}\right)_{t h e o r y}$ as a function of angle, $\theta_{t}$, for several FOVs for a curved tube $\left(T_{E}=2.06 \mathrm{~ms}\right)$. The thick solid line shows the theoretical value calculated for Eq. 2.113. The error bars represent the standard error calculated from the linear regression. . . . . . . . . . . . 94 
$4.8\left(\lambda_{\phi}\right)_{e x p} /\left(\lambda_{\phi}\right)_{t h e o r y}$ as a function of angle, $\theta$, for several FOVs for a thin walled straight tube parallel to $B_{0} \hat{z}$, with $T_{E}=5.48 \mathrm{~ms}$. The thick solid line shows the theoretical calculation from Eq. 2.113. The error bars represent the standard error calculated from the linear regression.

$4.9\left(\lambda_{\phi}\right)_{\text {exp }} /\left(\lambda_{\phi}\right)_{\text {theory }}$ as a function of angle, $\theta$, for several FOVs for a thin walled straight tube parallel to $B_{0} \hat{z}$, with $T_{E}=2.06 \mathrm{~ms}$. The thick solid line shows the theoretical calculation from Eq. 2.113. The error bars represent the standard error calculated from the linear regression. 96

$4.101 / T_{1}$ as a function of $[\mathrm{CA}]$ for the inversion recovery method and variable flip angle method. The linear fit for the VFA method data was $1 / T_{1}=(4.76 \pm 0.08) \mathrm{mM}^{-1} \mathrm{~s}^{-1} *[\mathrm{CA}]+(0.28 \pm 0.09) \mathrm{s}^{-1}$ with $\mathrm{R}=0.99874$ and $p<0.0001$. The error bars represent the standard error calculated from the parameter fit. . . . . . . . . . . . . . . . . .

5.1 3D rendering of the plastic tubing used to simulate a blood vessel, calculated from a 3D magnitude image. . . . . . . . . . . . .

5.2 2D representation of a $3 \mathrm{D}$ rendering of some of the draining veins of the brain segmented using Region Growing $3 D$ Segmentation (ImageJ). The upper portion is the SSS . . . . . . . . . . . . . . . 113

5.3 Profiles for a infinite cylinder of radius 16 pixels at angles of $0^{\circ}, 45^{\circ}$ and $90^{\circ}$ with respect to $B_{0} \hat{z}$. The orange line represents the theoretical profile and the black line represents the simulated profile. . . . . . 116

5.4 Profiles for an infinitely long cylinder of various radii and angles of a) $0^{\circ}$ and b) $45^{\circ}$ with respect to $B_{0} \hat{z}$. The cylinder was filled with a $1 \mathrm{mM}$ solution of CA and was surrounded by water. The theoretical $B_{d}(\mathbf{r})$ values for the inside an infinitely long cylinder and for the background are indicated in the graphs as dashed lines. . . . . . . . . . . 
5.5 The percent error in $B_{d}(\mathbf{r})$ versus radius for a single cylinder at $\theta=0^{\circ}$ (closed squares) and $\theta=45^{\circ}$ (open squares) with respect to $B_{0} \hat{z}$, for a matrix size of $512^{3}$. The cylinder was filled with a $1 \mathrm{mM}$ solution of CA and was surrounded by water. The linear fit for $\theta=0^{\circ}$ (orange line) was $y=(4.2174 \pm 0.0011) \times 10^{-4} x-(1 \pm 3) \times 10^{-5}, R=1, p<0.0001 .120$

5.6 Percent error in $B_{d}(\mathbf{r})$ for the equivalent radius for a matrix size of $512^{3}$. The linear fit for $\theta=0^{\circ}$ (orange line) was $y=(4.213 \pm 0.003) \times$ $10^{-5} x-(2 \pm 2) \times 10^{-4}, R=1, p<0.0001 \ldots \ldots \ldots \ldots \ldots 122$

5.7 Simulated $B_{d}(\mathbf{r})$ profile showing the effects of smoothing. . . . . . . 124

5.8 Histogram showing the ratio of the phase accumulation measured in experiments to that predicted by simulation. Total counts $\approx 200000$.

5.9 Profile of the phase accumulation measured in experiments to that predicted by simulation through a portion of tube where low phase accumulation contributes to high errors in $\Delta_{\text {exp }} / \Delta_{s i m} \ldots \ldots \ldots \ldots$

5.10 Profiles for two infinite cylinders at $90^{\circ}$ to $B_{0} \hat{z}$, each with a 16 pixel radius and $1 \mathrm{mM}$ of $\mathrm{CA} \ldots \ldots \ldots \ldots \ldots$

5.11 Profile along the $z$-axis for $B_{d}(\mathbf{r})$ for a cylinder 32 pixels long, a cylinder 256 pixels long, as well as the theoretical value for a sphere of diameter 32 pixels and a cylinder of infinite length. . . . . . . . . 130

5.12 One quadrant of the $B_{d}(\mathbf{r})$ map for a torus with a radius of curvature of 128 pixels and a radius of 16 pixels containing a $1 \mathrm{mM}$ solution of CA.132

5.13 Typical profiles for tori with various radii of curvature, each with a 16 pixel radius. For these profiles the tangent formed an angle of $0^{\circ}$ with $B_{0} \hat{z} \ldots \ldots \ldots \ldots \ldots \ldots \ldots \ldots \ldots \ldots \ldots$

5.14 The error in $[\mathrm{CA}]$ versus angle for several radii of curvature of the torus.133 
6.1 Typical DCE-MRI images for the same patient and slice; a) a $|S|$ image taken at maximum $[\mathrm{CA}]$ in the SSS b) a baseline $\phi$ image c) a $\phi$ image taken at maximum $[\mathrm{CA}]$ in the SSS. . . . . . . . . . . . . 149

6.2 Schematic of the 2D DCE-MRI protocol applied to the brain tumour study. a) A sagittal view of the brain shows the approximate slice location in the exam. Note that the blood flow through the SSS is in the superior to inferior direction. b) A typical image slice through the tumour and SSS. c) A typical "upper" neck slice showing the internal carotid artery (ICA), the external carotid artery (ECA) and the vertebral artery (VA). d) A typical "lower" neck slice showing the common carotid artery $(\mathrm{CCA})$ and the vertebral artery $(\mathrm{VA}) \ldots \ldots \ldots \ldots$. . .

6.3 Schematic of input/output function characterization parameters, peak height, area under the peak $\left(\mathrm{AUC}_{\text {peak }}\right)$ and washout. . . . . . 155

6.4 A typical $\mathrm{VOF}_{\phi}$ measured in the SSS before and after incorporating the geometry factor into the phase to $[\mathrm{CA}]$ conversion. Slices order from most inferior (I) to most superior $(\mathrm{S}) \ldots \ldots \ldots \ldots \ldots$

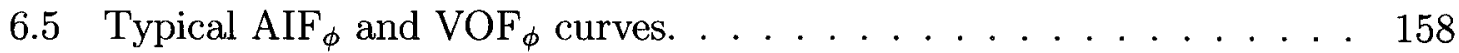

6.6 Typical $\mathrm{VOF}_{|S|}$ and $\mathrm{VOF}_{\phi}$ curves for each slice through the tumour. Slices order from inferior (I) to superior (S). Note that the graphs do not show the full measured time course. . . . . . . . . . . . .

6.7 $\mathrm{VOF}_{|S|}$ and $\mathrm{VOF}_{\phi}$ averaged over 28 cases compared to a population AIF from the literature. . . . . . . . . . . . . . . . 159

$6.8 K^{\text {trans }}$ and $v_{p}$ maps computed using $\mathrm{AIF}_{\phi}$ for a grade IV tumour. . . 161

$6.9 K^{\text {trans }}$ and $v_{p}$ from $\mathrm{AIF}_{\phi}$ versus $\mathrm{VOF}_{\phi}$ and $\mathrm{VOF}_{|S|}$ versus $\mathrm{VOF}_{\phi}$. Error bars on the graph represent one SD in the parameter over the whole ROI for each patient. The dashed line shows the line with slope $=1$. 
7.1 Schematic of the 3D DCE-MRI protocol applied to the prostate tumour study. a) A coronal view of the male pelvis shows the approximate slice location in the exam. Note that the blood flow through the artery is in the superior to inferior direction. b) A typical image slice through the prostate, showing the iliac artery (IA) and the obturator internus

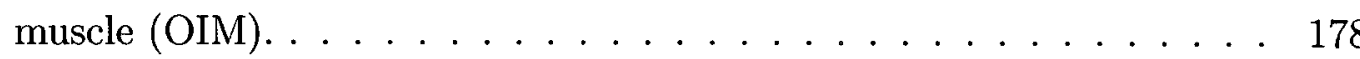

7.2 $\operatorname{AIF}_{|S|}(z)$ and $\operatorname{AIF}_{\phi}(z)$ for each slice block. Slice block 1 is most inferior and slice block 7 is most superior. Note that the full time course is not shown. . . . . . . . . . . . . . . . . . . 183

7.3 Perfusion parameters for the OIM resulting from $\operatorname{AIF}_{|S|}(z)$ (open circles) and $\operatorname{AIF}_{\phi}(z)$ (solid triangles) as a function of slice group. Slice block 1 is the most inferior block . . . . . . . . . . . . . . . 184

7.4 Optimal $\mathrm{AIF}_{|S|}$ and Optimal $\mathrm{AIF}_{\phi}$ averaged over 27 cases compared to two population AIF from literature. Note: the entire time course is not shown. . . . . . . . . . . . . . . . . . . .

7.5 Perfusion parameter results for the OIM calculated using $\mathrm{AIF}_{|S|}$ and $\mathrm{AIF}_{\phi}$ as well as two population AIFs from the literature. Data points show values for each patient. To the right of each distribution, the inter-patient mean and SD are shown. . . . . . . . . . . . . 186

$7.6 K^{\text {trans }}, v_{e}$ and $v_{p}$ computed using $\mathrm{AIF}_{\phi}$ for a typical prostate case. . 188

$7.7 K^{\text {trans }}$ and $v_{p}$ and $v_{e}$ measured using $\mathrm{AIF}_{|S|}$ versus $\mathrm{AIF}_{\phi}$. Error bars on the graph represent one SD in the parameter over the whole ROI for each patient. . . . . . . . . . . . . . . . . 


\section{Nomenclature}

Vector quantities are indicated with a bold typeface throughout the thesis.

\section{Symbol and Acronyms Definitions}

AIF Arterial Input Function; see also $C_{a}(t)(\mathrm{mM}) \ldots \ldots \ldots \ldots \ldots \ldots$

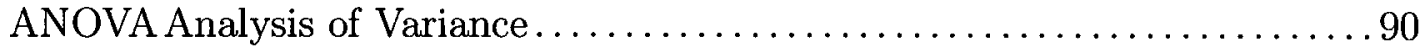

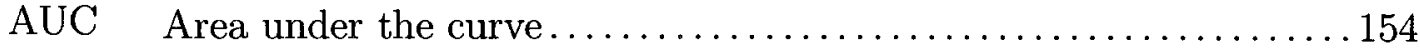

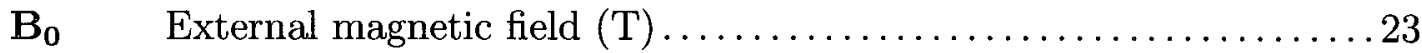

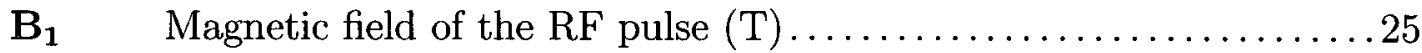

$\mathbf{B}_{\mathbf{M}} \quad$ Magnetic field shift induced by the presence of a material $(\mathrm{T}) \ldots \ldots 48$

BBB Blood Brain Barrier .................................. 14

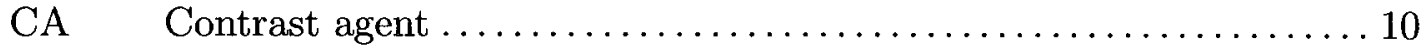

[CA] Concentration of contrast agent.......................

$C_{a} \quad$ Concentration of CA in the arterial whole blood (mM) $\ldots \ldots \ldots \ldots 11$

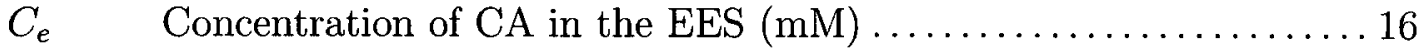

$C_{p} \quad$ Concentration of CA in the blood plasma $(\mathrm{mM}) \ldots \ldots \ldots \ldots \ldots$

$C_{p a} \quad$ Concentration of CA in the arterial blood plasma $(\mathrm{mM}) \ldots \ldots \ldots 13$

$C_{p v} \quad$ Concentration of $\mathrm{CA}$ in the venous blood plasma $(\mathrm{mM}) \ldots \ldots \ldots 13$

$C_{t} \quad$ Concentration of CA in the tissue $(\mathrm{mM}) \ldots \ldots \ldots \ldots \ldots \ldots \ldots \ldots \ldots$

$C_{v} \quad$ Concentration of $\mathrm{CA}$ in the venous whole blood $(\mathrm{mM}) \ldots \ldots \ldots 11$

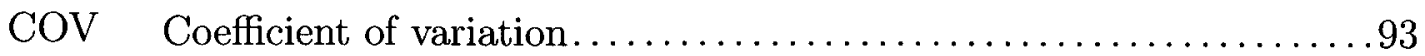

CT $\quad$ Computed Tomography $\ldots \ldots \ldots \ldots \ldots \ldots \ldots \ldots \ldots \ldots \ldots \ldots \ldots$ 


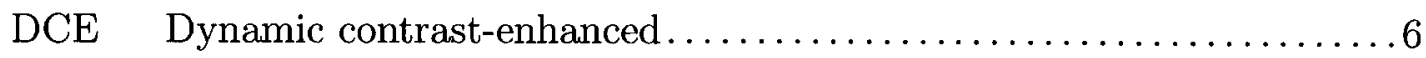

E Extraction fraction or Extraction ratio...................... 19

EES Extravascular Extracellular Space.......................... 7

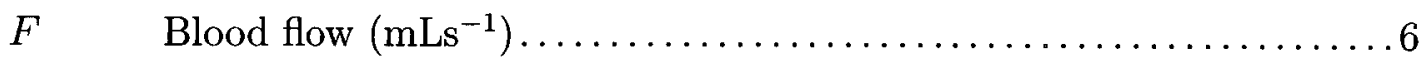

$F_{p} \quad$ Plasma blood flow $\left(\mathrm{mLs}^{-1}\right) \ldots \ldots \ldots \ldots \ldots \ldots \ldots \ldots \ldots \ldots \ldots \ldots \ldots \ldots \ldots \ldots$

FOV Field Of View $\ldots \ldots \ldots \ldots \ldots \ldots \ldots \ldots \ldots \ldots \ldots \ldots \ldots \ldots \ldots$

G Gradient in magnetic field per unit distance $\left(\mathrm{Tm}^{-1}\right) \ldots \ldots \ldots \ldots 31$

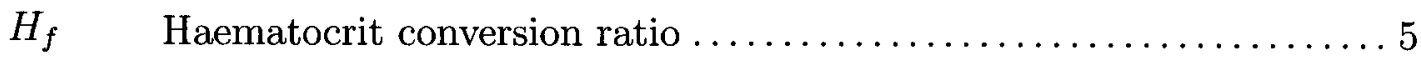

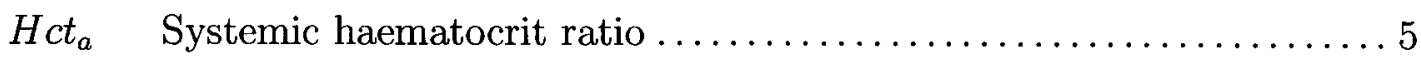

$H c t_{m} \quad$ Microvascular haematocrit ratio $\ldots \ldots \ldots \ldots \ldots \ldots \ldots \ldots \ldots \ldots$

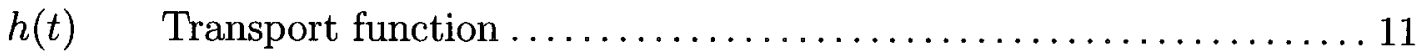

$K^{\text {trans }}$ Transfer constant $\left(\min ^{-1}\right) \ldots \ldots \ldots \ldots \ldots \ldots \ldots \ldots \ldots \ldots \ldots \ldots \ldots \ldots \ldots \ldots \ldots$

M Macroscopic magnetization $\left(\mathrm{Am}^{-1}\right) \ldots \ldots \ldots \ldots \ldots \ldots \ldots \ldots \ldots \ldots \ldots \ldots \ldots \ldots \ldots$

$M_{0} \quad$ Magnitude of magnetization $\mathbf{M}$ at thermal equilibrium..........27

$M_{x y} \quad$ Transverse component of magnetization $\mathbf{M} \ldots \ldots \ldots \ldots \ldots \ldots 27$

$M_{z} \quad$ Longitudinal component of magnetization $\mathrm{M} \ldots \ldots \ldots \ldots \ldots \ldots 27$

MTT Mean Transit Time.................................. 12

NMR Nuclear Magnetic Resonance ........................... 22

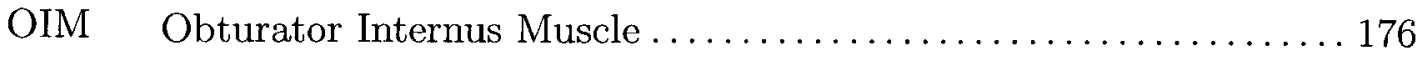

$P S \quad$ Permeability surface area product $\left(\mathrm{mLs}^{-1}\right) \ldots \ldots \ldots \ldots \ldots \ldots \ldots$

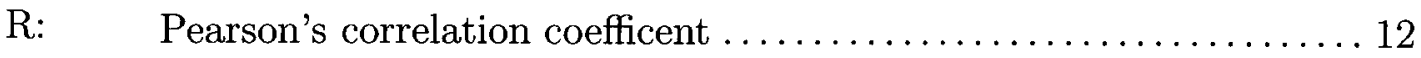

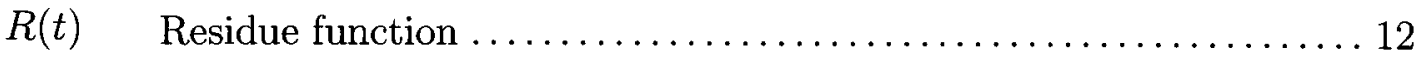

ROI Region of interest................................ 84

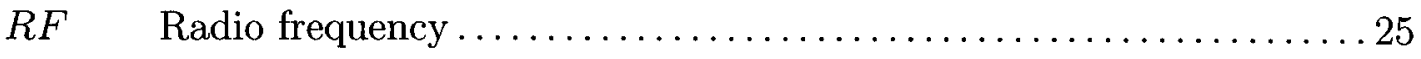

$|S| \quad$ Magnitude of MRI signal (A.U.) ........................ 35

$S_{I} \quad$ Imaginary component of MRI signal (A.U.) $\ldots \ldots \ldots \ldots \ldots \ldots \ldots \ldots$

$S_{R} \quad$ Real component of MRI signal (A.U.) ................... 35 


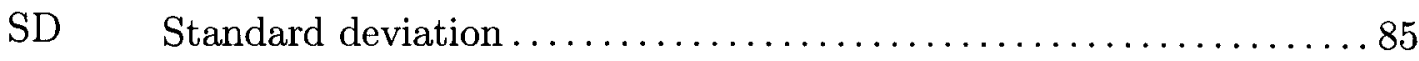

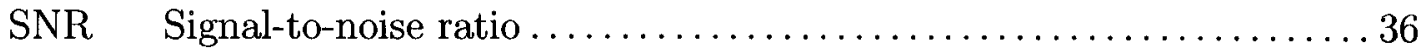

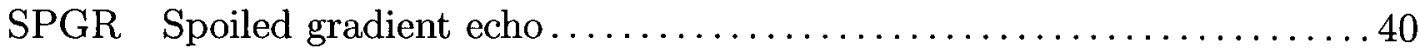

SSS Superior Sagittal Sinus ............................... 149

$T_{1} \quad$ Longitudinal relaxation time $(\mathrm{ms}) \ldots \ldots \ldots \ldots \ldots \ldots \ldots \ldots \ldots \ldots \ldots \ldots$

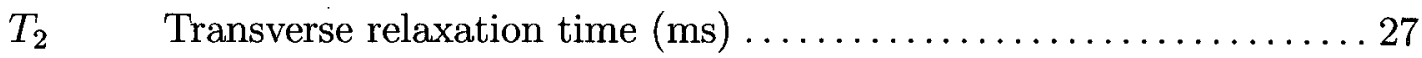

$T_{2}^{*} \quad T_{2}$ observed in a gradient echo sequence $(\mathrm{ms}) \ldots \ldots \ldots \ldots \ldots \ldots . \ldots 29$

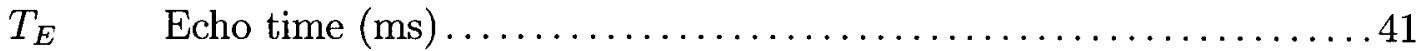

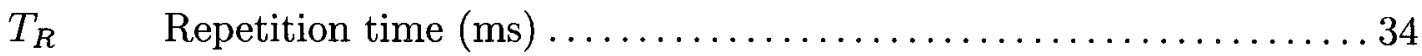

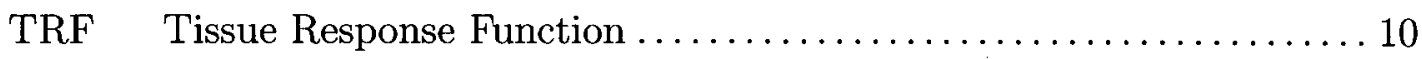

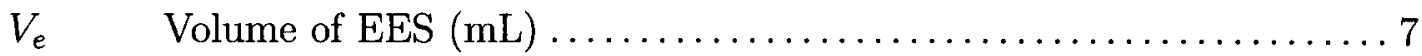

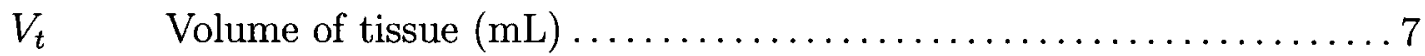

$V_{p} \quad$ Volume of blood plasma $(\mathrm{mL}) \ldots \ldots \ldots \ldots \ldots \ldots \ldots \ldots \ldots \ldots$

$V_{d} \quad$ Volume of distribution of the CA $(\mathrm{mL}) \ldots \ldots \ldots \ldots \ldots \ldots \ldots \ldots$

VFA Variable Flip Angle...................................62

VOF Venous Output Function; see also $C_{v}(t)(\mathrm{mM}) \ldots \ldots \ldots \ldots \ldots \ldots \ldots 11$

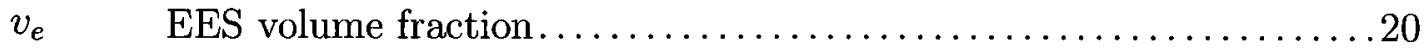

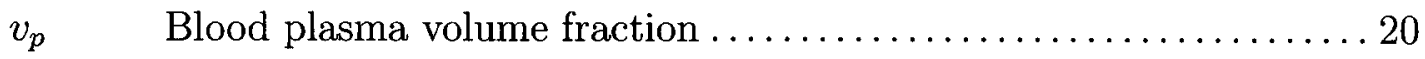

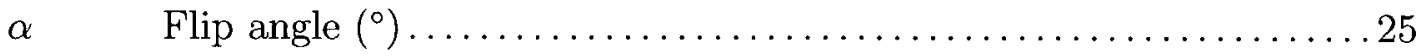

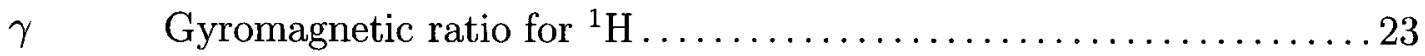

$\theta \quad$ Angle between $\mathbf{B}_{\mathbf{0}}$ and the $z$-axis of the object coordinate system . . 45

$\sigma_{g} \quad$ Standard deviation of signal from a single receiver channel........ 35

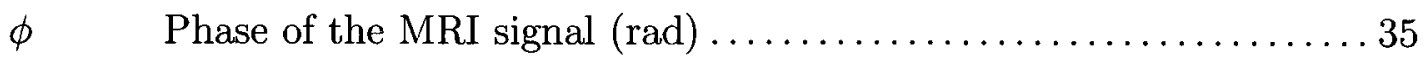

$\chi \quad$ Magnetic susceptibility .............................. 44

$\chi_{m} \quad$ Molar magnetic susceptibility $\left(\mathrm{M}^{-1} \ldots \ldots \ldots \ldots \ldots \ldots \ldots \ldots \ldots \ldots \ldots \ldots \ldots \ldots\right.$

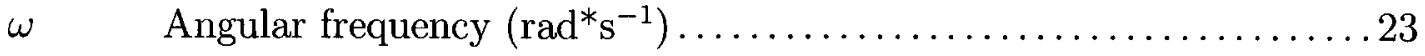




\section{Chapter 1}

\section{Introduction}

The delivery and removal of nutrients is essential to the well-being of living tissue. The delivery of nutrients into a tissue via the capillary bed is termed tissue perfusion. Knowledge of tissue perfusion can provide useful information about the physiological state of the tissue. More specifically, studies of tissue perfusion aim to provide information about blood delivery and capillary permeability in order to gain insight into nutrient transport to the tissue as well as other important physiological processes such as angiogenesis. Absolute measures of parameters related to perfusion, such as blood flow, blood volume and the volume transfer coefficient $\left(K^{\text {trans }}\right)$, provide important diagnostic and prognostic information relevant to diseases such as cancer. For example, the rapid growth of tumour cells causes improper development of the tumour's vascular structure which results in measurable changes in the tissue perfusion parameters for these tissues compared to healthy tissue. This information is useful for many things including in tumour grading, as a clinical endpoint for drug treatments and in assessing damage to the blood brain barrier.

Tissue perfusion parameters can be measured non-invasively using dynamic contrast-enhanced magnetic resonance imaging (DCE-MRI). With this technique a small amount of contrast agent (CA) is injected as an intravenous bolus. The CA is pumped, by the heart, through the vascular system and into the capillaries where, 
given the right combination of physiological factors and CA size, it will diffuse into the extravascular space of the tissue. Some CA's used with other imaging modalities can enter cells, but in MRI, the CA's currently in use remain extracellular. Images of the volume of interest are taken repeatedly throughout the passage of the CA in order to track its delivery to the tissue. The presence of the CA alters the contrast in the image which enables the determination of the CA concentration, denoted [CA], and, by using tracer-kinetic modelling, allows the tissue perfusion parameters to be determined. Clinical studies then relate these parameters to pathophysiological processes through comparison with clinical endpoints and gold standard methods.

There are many different approaches to data acquisition and analysis in the measurement of tissue perfusion using MRI. For absolute quantification of perfusion parameters with any of these methods, accurate measurements of [CA] in both the tissue of interest and in a feeding artery are required. The latter is referred to as the arterial input function or AIF. In DCE-MRI, the signal which forms the image is not directly proportional to [CA]. Current methods for the conversion of the MR signal to [CA] involve the use of the magnitude of the MR signal. The MRI images one normally encounters are formed from the magnitude of the signal, but since the MR signal is a vector, phase information is also available. If the geometry of the vessel is known, the phase of the MR signal can be used to calculate [CA] through a linear scaling factor. There are several advantages to using the phase rather than the magnitude: the phase is linear with [CA] and it does not saturate at high concentrations, it is insensitive to flip angle variations, and it is less sensitive to the effects of blood flow. The purpose of this work was to explore the feasibility of using the MR signal phase to measure $[\mathrm{CA}]$ in blood vessels to obtain a more accurate AIF for perfusion modelling.

The study of perfusion using MRI is a multi-disciplinary subject mixing physiology, tracer kinetic modelling and MR physics. Chapter 2 of the thesis will focus on 
familiarizing the reader with the theory needed to understand the rationale and context for the approach taken in the proceeding chapters. First an introduction into the physiology of perfusion and tumour growth will be given. Next, in order to facilitate a discussion of the clinical results presented in Chapters 6 and 7, the modelling used to extract the perfusion parameters will be described. Following this, the relevant MR physics needed to understand the challenges being addressed in this thesis and the approach taken to solve them will be presented. Finally, methods for the conversion from signal magnitude and phase to $[\mathrm{CA}]$ are discussed. Following this presentation of background material, the motivation for this work and the goals of this thesis are discussed in detail in Chapter 3.

The main goal of this thesis is to show that the use of the phase of the MR signal, rather than the magnitude, improves the quantification of the perfusion parameters in DCE-MRI studies. The work towards this goal will be presented in four parts, organized into chapters. In Chapters 4 and 5 the results of in vitro experiments and simulations are presented to establish and validate the technique. The application of the method to clinical brain and prostate tumour studies is discussed in Chapters 6 and 7 , respectively. These clinical studies, along with results from the in vitro experiments and simulations, provide evidence for the value of the MR signal phase in the calculation of the contrast agent concentration in the AIF. The thesis objectives are addressed in considerably more detail in Chapter 3, after the appropriate background has been introduced. 


\section{Chapter 2}

\section{Theory \& Background}

\subsection{Tissue Perfusion}

The vascular system is responsible for transporting oxygen and nutrients to the tissues of the body, a process called tissue perfusion. The efficiency of the vascular system at this task is governed primarily by capillary blood flow, capillary density and transcapillary exchange. The measurement of these properties can reveal important characteristics of the tissue. For instance, rapidly and poorly formed capillaries such as those found in many tumours, tend to have higher permeabilities than normally formed capillaries. This information could have use clinically in the diagnosis of cancer as well as for the prediction and monitoring of tumour treatment outcome. Section 2.1.1 describes the vascular system in more detail and section 2.1.2 outlines the expected perfusion characteristics of tumours.

\subsubsection{The Vascular System}

Nutrient- and oxygen-rich blood flows from the heart through large blood vessels called arteries. The arteries subdivide into smaller vessels called arterioles. As the arterioles enter the tissue they branch into numerous tiny vessels called capillaries. The thin walls of the capillaries allow the exchange of substances between the blood 
and the extravascular space of the tissue. Capillaries form extensive branching networks to increase the total surface area available for exchange. Groups of capillaries re-converge to form venules which in turn combine to form veins (see Fig. 2.1). In addition to supplying nutrients to the tissue, the blood also carries away waste and carbon dioxide through the veins back to the heart and lungs and for further processing by the kidneys and liver. The capillaries make up the vascular compartment of the tissue.

The vascular compartment can be further subdivided into two sub-compartments: (1) the blood plasma and (2) the formed elements (cells and cell fragments) [1, p 667]. The blood plasma comprises $55 \%$ of the total blood volume and the formed elements, consisting almost entirely of red blood cells (RBCs), make up the rest. The percentage of the total blood volume occupied by the RBCs is called the haematocrit ratio $(H c t)$. The normal haematocrit ratio range for adult females is $38-46 \%$ and for adult males is 40-54\%. An increase in Hct (called polycythemia) can be caused by tissue hypoxia $[1, \mathrm{p} 669]$. The haematocrit ratio in the capillaries, called the microvascular haematocrit ratio, $H_{c t}$, is not the same as that in the larger blood vessels, called the systemic haematocrit ratio, $H c t_{a}$. The difference depends on vessel size, flow and pathophysiology. In this work $H c t_{a}$ and $H c t_{m}$ where taken to be 0.45 and 0.25 respectively [2]. To simplify notation, $H_{f}$, which is a haematocrit factor accounting for this difference is defined as [2]

$$
H_{f} \equiv \frac{1-H c t_{a}}{1-H c t_{m}}
$$

can also be used.

Nutrients and oxygen are exchanged between the vascular compartment and the interstitial fluid of the tissue $[1, \mathrm{p} 744]$. In turn, substances are also exchanged between 
the interstitial fluid and the cells. Exchange of substances between these compartments can occur via diffusion down the concentration gradient of the substance, as is the case with the exchange of MRI contrast agents. Other mechanisms of exchange exist but, since the contrasts agents used in this research do not participate in these types of exchange, they are not discussed further in the thesis. Capillaries in different tissues allow specific substances to pass depending on the function of the tissue. For example, in the brain, the vascular-interstitial fluid boundary, more commonly known as the blood brain barrier, restricts the transfer of the MRI contrast agents used in this research, whereas most other tissues allow it to pass [3].

The exchange of nutrients between capillaries and their surroundings depends on the permeability, $P$, of the capillaries as well the surface area, $S$, available for exchange [4]. The permeability and surface area cannot be measured independently at the resolution available in MRI and thus are always measured as a product, $P S$ $\left(\mathrm{mLs}^{-1}\right)$. Exchange is also affected by the microcirculation or capillary blood flow, $F$, which describes the volume of whole blood passing through the capillaries in the tissue per unit time $\left(\mathrm{mLs}^{-1}\right)$.

The number of capillaries in a tissue varies with the metabolic activity of the tissue they serve $[1, \mathrm{p} 739]$. The muscles, liver, kidneys and nervous system use more oxygen and nutrients than other tissues and thus require a greater number of capillaries. Tendons and ligaments have lower metabolic requirements and thus contain fewer capillaries. The average capillary density in human skeletal muscle is about 600 per $\mathrm{mm}^{3}$ [5]. Due to their small size, capillaries, which range in diameter from 4 to $10 \mu \mathrm{m}$ [1, p 739], are not individually resolvable using MRI. The perfusion parameters derived from dynamic contrast-enhanced MRI (DCE-MRI) studies are thus a measure of the bulk tissue properties of each tissue voxel (volume element). Because of this, the detailed microscopic structure of the tissue is often ignored.

To facilitate the discussion of perfusion modelling to follow, a simplified model of 
the tissue will be presented. The tissue voxel is divided into vascular and extravascular compartments which each are further subdivided into cellular and non-cellular compartments: the interstitial fluid or extravascular extracellular space (EES), $V_{e}$, and the cellular space, $V_{\text {cell }}$, form the extravascular compartments while the blood plasma volume, $V_{p}$, and the red blood cell volume, $V_{R B C}$, form the vascular compartments, giving a total volume, $V_{t}$. This is summarized in the following equation:

$$
V_{e}+V_{\text {cell }}+V_{p}+V_{R B C}=V_{t}
$$

The choice for these compartments originates from the properties of the MRI contrast agent [6]. The contrast agent is only present in the plasma component of the blood and the EES because it cannot penetrate cellular membranes. The total volume available to the contrast agent is referred to as the volume of distribution, $V_{d}$. Note that the blood plasma volume in the microvasculature can also be related to the total blood volume, $V_{b}$, by

$$
V_{p}=\left(1-H c t_{m}\right) V_{b}
$$

Similarly, to avoid ambiguity a distinction between whole blood flow in the microvasculature, $F$, and plasma blood flow, $F_{p}$, should be made. The two can be related by the microvascular haematocrit ratio,

$$
F_{p}=F\left(1-H c t_{m}\right)
$$

\subsubsection{Tumour Development and Perfusion Characteristics}

Sometimes normal cells in the body begin to divide in an uncontrolled manner resulting in cancerous cells which can accumulate to form a solid mass called a tumour. 


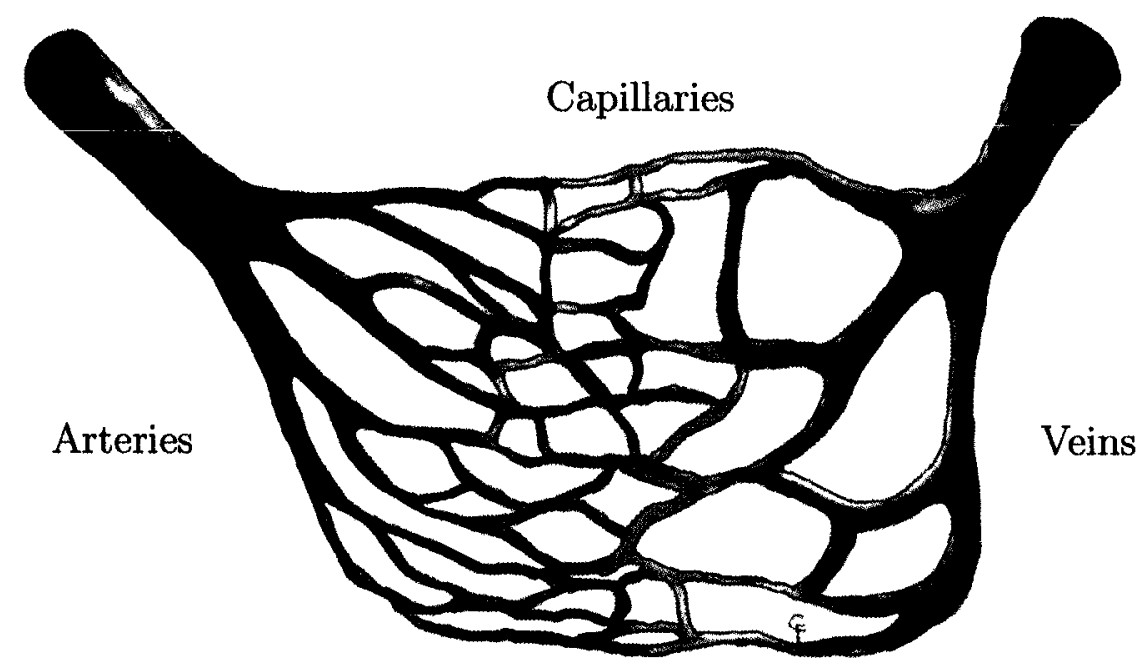

Figure 2.1: The vascular system transports nutrients and oxygen to the tissue and removes waste and carbon dioxide from the tissue.

The nutrient supply available to a tumour will affect its ability to grow. Thus, the measurement of tissue perfusion parameters in a tumour, which are indicative of its nutrient supply, can provide valuable information for clinical decision making [7]. These perfusion parameters can be used clinically to assess antiangiogenic and antivascular drugs, to grade tumours, to guide biopsy or to differentiate recurrent tumour growth from treatment-related necrosis [8].

In the early stages of tumour development, cell division usually occurs avascularly [9]. That is, the tumour is not fed directly by blood vessels, but rather receives its nutrition via diffusion through the interstitial fluid. At this stage, the tumour size is limited to a few millimeters [10]. Once the tumour has reached this limit, it is subjected to increased hypoxic stress (lack of oxygen), one of the primary stimuli for new vessel growth [11]. As a result, it may secrete proteins called tumour angiogenic growth factors, which stimulate the growth of new blood vessels (angiogenesis) to nourish the tumour. One such protein, the vascular endothelial growth factor (VEGF), appears to be a major regulator in tumour angiogenesis [7]. 
With rapidly growing cancer cells, tumour angiogenesis typically results in blood vessels that are formed more rapidly than those in healthy tissue. As a result of their rapid formation, the capillaries can be malformed and the vessel walls are often highly permeable [10]. Thus the area surrounding a tumour tends to have high vascular density and high vascular permeability. The resulting perfusion properties of tumours can differ significantly from those of normal tissue. The size of the difference appears to be dependent on the malignancy of the tumour [7]; the capillaries surrounding more aggressive tumours tend to show higher and more heterogeneous permeability. Several recent studies have shown that quantitative estimates of microvascular permeability correlate with brain tumour grade [8].

There can also be areas of a tumour where the contrast agent injected into the patient in a DCE-MRI exam does not reach, often at the center of the tumour. These areas may be hypoxic, meaning that the oxygen supply is insufficient to meet the demands of oxygen consumption. Hypoxic tissue is usually found in areas with a ratio of microvascular density to cellular density which is lower than that found in healthy tissue [12]. The presence of hypoxic regions in the tumour is associated with poor prognosis and resistance to radiation therapy [13].

These heterogeneities in tumour microvasculature lead to variations in the enhancement patterns seen in perfusion studies such as those reported in this thesis. There could be uniform enhancement or heterogeneous enhancement, increased or decreased enhancement and faster or slower enhancement. These patterns of enhancement result from combinations of the various perfusion properties described in the previous section, and in order to derive the most physiologically relevant conclusions from them, a system must exist to facilitate their comparison. One way to do this is to apply a model to the enhancement pattern based on the kinetics of the injected contrast agent. 


\subsection{Modelling Tissue Perfusion}

The focus of this section is the modelling used to connect the clinical study to the desired perfusion parameters, without making reference to how the $[\mathrm{CA}]$ is measured. In Section 2.2.1 the inputs to the perfusion models acquired from a typical perfusion dataset are defined. A general approach to perfusion modelling is presented in Section 2.2.2. The general model is used to define three perfusion parameters: blood flow, blood volume and mean transit time. The remaining sections focus on modelling transcapillary exchange.

\subsubsection{Tissue Perfusion Data}

The goal of dynamic contrast-enhanced magnetic resonance imaging (DCE-MRI) is to use the acquired images to determine the perfusion properties of the tissue. To do this, a substance which provides contrast in the MRI images, a contrast agent (CA), is injected into the patient. Multiple images are acquired as the CA passes through the capillaries into the tissue. The change in intensity in each image voxel is proportional to the change in the concentration of the $\mathrm{CA}([\mathrm{CA}])$ in the associated tissue volume element, as a function of time. This time course is referred to as a tissue response function (TRF) (see Fig. 2.2).

If the CA could be injected in the form of an instantaneous bolus, the resulting TRF would be a direct reflection of the perfusion parameters. The CA, however, cannot be delivered instantaneously. As a consequence, the amount of CA entering the tissue will vary in time. The shape of the TRF is affected by the delivery of blood to the tissue and, therefore, also depends on the characteristics of the input function. For this reason, the input into the tissue must also be measured. The input is commonly measured in a nearby artery and is referred to as the arterial input function (AIF). In cases where the AIF cannot be measured with sufficient accuracy 


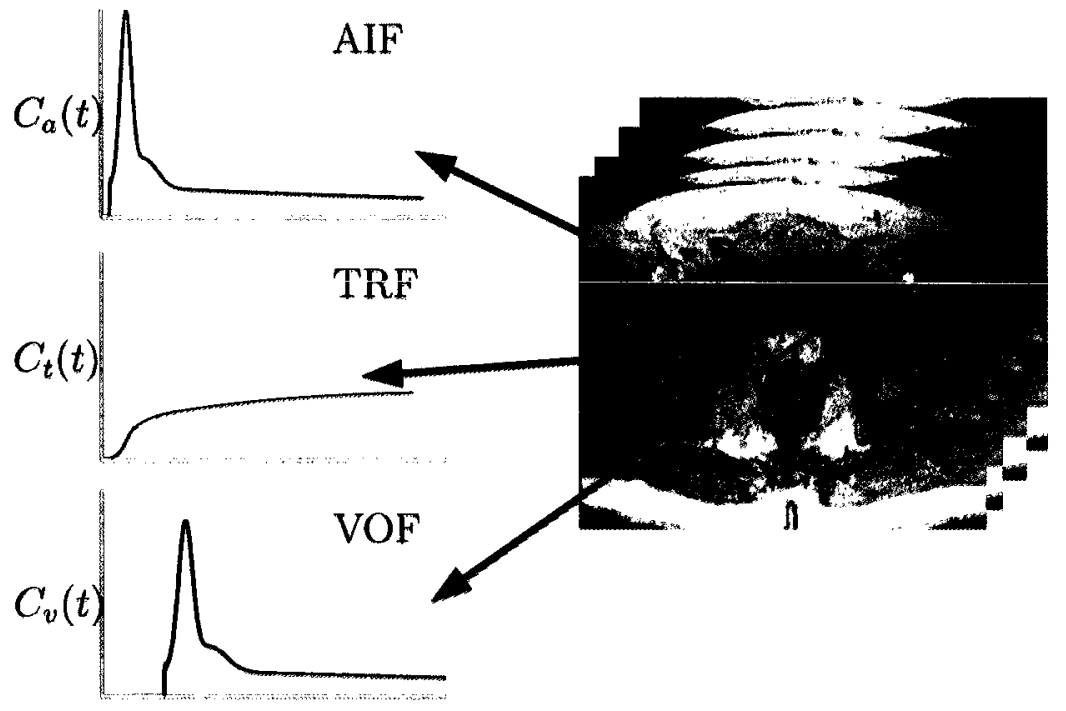

Figure 2.2: A schematic of information extracted from a dynamic contrast-enhanced (DCE-MRI) exam. The arterial input function (AIF), the tissue response function (TRF) and the venous output function (VOF) are the experimental inputs used in DCE-MRI modelling. $C_{a}, C_{t}$ and $C_{v}$ are the [CA] in the arterial whole blood, the tissue and the venous whole blood.

an additional measurement of the output of the system, the venous output function (VOF), is also made.

\subsubsection{General Approach to Perfusion Modelling}

There are several approaches one could take for the development of a model to the tissue perfusion properties to the observed TRF. The fundamental principle of all tissue perfusion models is that the total amount of CA entering the tissue is equal to the total amount leaving the tissue. The simplest case is to consider the entire volume of distribution of the $\mathrm{CA}$ as one compartment. That is, to combine the blood plasma and EES into one compartment. No assumptions about the nature of the volume are necessary at this point.

Begin by defining a transport function, $h(t)$, as the probability density function (pdf) for CA molecule transit times through the system (molecules* $\mathrm{s}^{-1}$ )[14]. Since 
the function is a pdf, it is normalized such that

$$
\int_{0}^{\infty} h(\tau) \mathrm{d} \tau=1
$$

Thus, $h(\tau) d \tau$ is the fraction of molecules that take between $\tau$ and $\tau+\mathrm{d} \tau$ to pass through the system. The transport function can also be interpreted as the time at which the CA molecules leave the capillary network, for the special case where all of the CA molecules enter the system at exactly the same time (i.e. the input function is a delta function). If we also assume that all molecules travel at the same rate, the transport function for this special case would be a delta function, $\delta\left(\tau_{t}\right)$, where $\tau_{t}$ is the transit time. For more realistic vascular structures, the shape of the transport function will vary since capillaries in the voxel will have different lengths and widths and the exchange of CA between the vascular compartment and the EES must also be considered. Given that the microvascular structure is complex, a range of transit times can be expected for a given voxel. The mean time for a CA molecule to pass through the volume of distribution (defined in Section 2.1.1), referred to as the mean transit time (MTT), is the first moment of the transport function [14]:

$$
M T T \equiv \int_{0}^{\infty} \tau h(\tau) \mathrm{d} \tau
$$

One can also measure the amount of $\mathrm{CA}$ remaining in the tissue as a function of time. This is described by the residue function, $R(t)$ [14], which is defined as the probability that a $\mathrm{CA}$ molecule entering the tissue at $t=0$ will still be in the voxel at time $t$ and is related to the transport function through the following equation [14]:

$$
R(t)=1-\int_{0}^{t} h(\tau) \mathrm{d} \tau
$$

It is a monotonically non-increasing function with limits $R(0)=1$ and $R(\infty) \rightarrow 0$. 
The latter condition indicates complete removal (washout) of the CA after some long time interval. The MTT can also be determined from the area under the curve of the residue function $[15, \mathrm{p} 271]$,

$$
M T T=\int_{0}^{\infty} R(\tau) \mathrm{d} \tau
$$

The transport function defined above describes the behaviour of the CA in response to an ideal impulse input (i.e. instantaneous bolus of tracer). It is not possible however, to achieve an ideal injection in practise. The shape of the input function must also be considered in the perfusion analysis. Assume that the $[\mathrm{CA}]$ in the input arterial blood plasma is given by the function $C_{p a}(t)$. The concentration in the venous blood plasma, $C_{p v}(t)$, after the CA has traversed the voxel will be given by the convolution of the input function and the transport function [14]:

$$
C_{p v}(t)=C_{p a}(t) \otimes h(t)=\int_{0}^{t} C_{p a}(\tau) h(t-\tau) \mathrm{d} \tau
$$

\section{The Tissue Response Function}

Next consider the amount of CA remaining in the tissue. The amount of CA in the voxel at any given time, $A_{t}(t)$, is determined by how much CA flows into the voxel per unit time and how long it remains in the voxel, defined by $R(t)$. Assuming a constant blood flow, the amount of CA entering the voxel is equal to $C_{p a}(t)$ times the volume of blood plasma entering the tissue per unit time, $F_{p}$. Then [14],

$$
\begin{aligned}
A_{t}(t) & =F_{p} C_{p a}(t) \otimes R(t) \\
& =F_{p} \int_{0}^{t} C_{p a}(\tau) R(t-\tau) \mathrm{d} \tau .
\end{aligned}
$$

From Eq. 2.11, solving for the measured quantity, TRF, or equivalently the [CA] 
in the whole tissue, $C_{t}(t)$, gives

$$
C_{t}(t)=\frac{F_{p}}{V_{t}} C_{p a}(t) \otimes R(t)
$$

This can be rewritten in terms of the measured AIF $\left(C_{a}(t)\right)$ using the relations from Section 2.1.1 to give

$$
C_{t}(t)=\frac{F}{V_{t} H_{f}} C_{a}(t) \otimes R(t)
$$

It is also common in the literature to find blood flow expressed per unit mass of the tissue $\left(\mathrm{mLs}^{-1} \mathrm{~g}^{-1}\right)$. To avoid confusion this will be labeled $F^{\prime}$. This leads to the following form of Eq. 2.13,

$$
C_{t}(t)=\frac{\rho F^{\prime}}{H_{f}} C_{a}(t) \otimes R(t)
$$

In a perfusion study, a deconvolution can be performed on the measured dataset to separate $C_{a}(t)$ from $\frac{F}{V_{t} H_{f}} R(t)$. By applying the boundary condition $R(0)=1$ the blood flow can then be extracted [14]. An alternative approach to the deconvolution method is to assume an analytic expression for $R(t)$. This introduces assumptions about the microvasculature but has the advantage that the model can be more stable because it reduces the number of degrees of freedom [14].

This general model can be applied directly in cases where the CA remains intravascular and the volume of distribution is equal to the plasma vascular volume. That is to say, the contrast molecules traverse the voxel without diffusing from the capillaries into the EES. This is true, for example, in the brain when the blood brain barrier (BBB) is intact, as the BBB does not allow the MRI CA to leave the microvasculature [3]. In the case where the CA does not remain intravascular the functional form of $R(t)$ is defined explicitly by modelling of transcapillary exchange. 


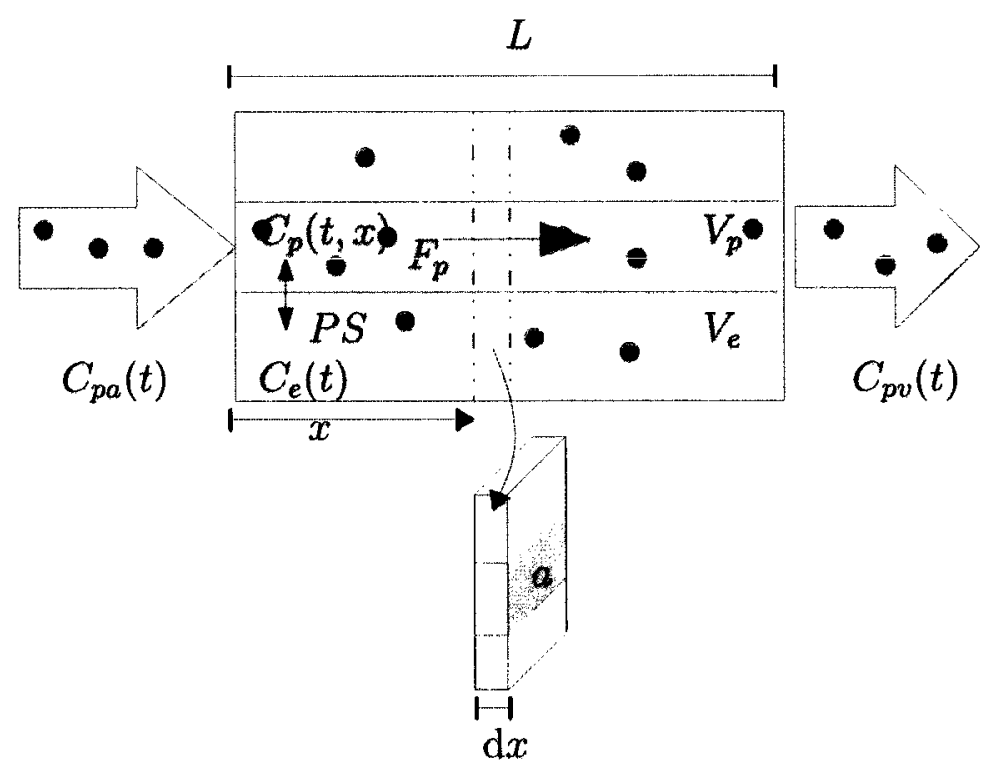

Figure 2.3: Simplified tissue model

\subsubsection{Modelling Transcapillary Exchange}

The MRI CA will leak out of the vascular space into the interstitial fluid (EES) in all tissues except healthy brain tissue, where the $\mathrm{BBB}$ is impermeable to the $\mathrm{CA}$ [3]. This means that a DCE-MRI study can, in principle, provide information about the permeability of the capillaries to the CA. To quantify the exchange of CA between the prasma in capillaries and the ERS, a mociei is sef up which consiciers the nux of $\mathrm{CA}$ between the two compartments under the assumption of free diffusion [3]. The rate of change of $\mathrm{CA}$ in each compartment is considered to be proportional to the difference in concentration between the two compartments. The tissue homogeneity model proposed by Johnson and Wilson [4] provides a good starting point to consider the majority of the models found in the DCE-MRI literature, although the original derivations of these models do not necessarily originate from the tissue homogeneity model equations.

The tissue homogeneity model assumes that the total vascular structure in a given 
voxel can be summed into one large vessel with total surface area, $S$, available for exchange. This vessel has a uniform permeability, $P$, which is the average permeability of the capillaries in the voxel. The vascular volume is assumed to have a length, $L$, and be of uniform cross-sectional area, $a$, along this length. The surface area per unit length of the vascular compartment is then $S / L$. The model assumes that there is a negligible concentration gradient along the cross section, although it allows a concentration gradient along the length. Diffusion within the interstitial space is assumed to be instantaneous, meaning that the compartment is well mixed. See Fig. 2.3 for a schematic diagram of the model.

Consider a small segment of the tissue of width $\mathrm{d} x$ at $x$. The amount of CA transfered from the vascular plasma compartment to the interstitial compartment between $t$ and $t+\mathrm{d} t$ is

$$
A_{e x}(t)=\frac{P S}{L} \mathrm{~d} x\left(C_{p}(x, t)-C_{e}(t)\right) \mathrm{d} t,
$$

where $C_{p}$ is the [CA] in the blood plasma in the microvasculature and $C_{e}$ is the [CA] in the EES. The amount of CA in the small vascular segment will also be affected by the flow into the segment between $t$ and $t+\mathrm{d} t$,

$$
A_{\text {in }}(t)=F_{p} C_{p}(x, t) \mathrm{d} t
$$

and the flow out of the segment between $t$ and $t+\mathrm{d} t$,

$$
A_{\text {out }}(t)=F_{p} C_{p}(x+\mathrm{d} x, t) \mathrm{d} t .
$$

Thus the change in the amount of CA in the small vascular segment is

$$
\mathrm{d} A_{p}(x, t)=A_{\text {in }}-A_{\text {out }}-A_{\text {ex }}
$$


Replacing $A_{p}(x, t)$ with the concentration times the volume of the vascular segment $\left(V_{p} \mathrm{~d} x / L\right)$ and dividing by $\mathrm{d} x$ gives

$$
\frac{V_{p}}{L} \mathrm{~d} C_{p}(x, t)=F_{p} \frac{C_{p}(x, t)-C_{p}(x+\mathrm{d} x, t)}{\mathrm{d} x} \mathrm{~d} t-\frac{P S}{L}\left(C_{p}(x, t)-C_{e}(t)\right) \mathrm{d} t .
$$

By taking the limit as $\mathrm{d} x$ becomes small, the final differential equation becomes

$$
\frac{V_{p}}{L} \frac{\partial C_{p}(x, t)}{\partial t}+F_{p} \frac{\partial C_{p}(x, t)}{\partial x}=-\frac{P S}{L}\left(C_{p}(x, t)-C_{e}(t)\right) .
$$

The change in concentration in the interstitial fluid between $t$ and $t+\mathrm{d} t$ is the sum of the amount of CA transfer per $\mathrm{d} x$ over the whole length of the capillary for this time. Therefore, from Eq. 2.15 it can be seen that

$$
V_{e} \frac{\mathrm{d} C_{e}(t)}{\mathrm{d} t}=\frac{P S}{L} \int_{0}^{L}\left(C_{p}(x, t)-C_{e}(t)\right) \mathrm{d} x
$$

Equations 2.20 and 2.21 are subject to the boundary conditions $C_{p}(x, t=0)=$ $C_{e}(t)=0$ and $C_{p}(x=0, t>0)=C_{p a}(t)$.

There is no closed form solution to these differential equations in time space, only in Laplace space. One cannot simply apply a Laplace transform to the concentration curves because the concentration does not go to zero within the imaging experiment time frame. It is not practical to continue imaging for that long; the patient would not be able to remain motionless.

Fortunately, further approximations can be introduced to the differential equations, based on the specific physiology and the imaging constraints (e.g. SNR and time resolution) used in a given study. The following sections outline some of the commonly used models. 


\subsubsection{Two Compartment Models}

First, consider the case where the $[\mathrm{CA}]$ in the vascular system is in a steady state while the exchange between the blood plasma and interstitial fluid is occurring. This amounts to assuming that for a given time, $t_{i}$,

$$
\frac{\partial C_{p}\left(x, t_{i}\right)}{\partial t}=0
$$

Applying this assumption, Eq. 2.20 simplifies to

$$
F_{p} \frac{\mathrm{d} C_{p}(x)}{\mathrm{d} x}=\frac{P S}{L}\left(C_{e}\left(t_{i}\right)-C_{p}\left(x, t_{i}\right)\right)
$$

which has the solution

$$
C_{p}\left(x, t_{i}\right)=C_{e}\left(t_{i}\right)+\left(C_{p}\left(0, t_{i}\right)-C_{e}\left(t_{i}\right)\right) \exp \left(\frac{-P S x}{F_{p} L}\right)
$$

where $C_{p}(x=0, t)=C_{p a}(t)$.

Inserting this result into Eq. 2.21 gives

$$
\begin{aligned}
V_{e} \frac{\mathrm{d} C_{e}(t)}{\mathrm{d} t} & =F_{p}\left(1-e^{\frac{-P S}{F_{p}}}\right)\left(C_{p a}(t)-C_{e}(t)\right) \\
& =K^{\text {trans }} V_{t}\left(C_{p a}(t)-C_{e}(t)\right),
\end{aligned}
$$

where $K^{\text {trans }}$, the volume transfer constant between the vascular input and the EES is defined as

$$
K^{\text {trans }} V_{t}=F_{p}\left(1-e^{-\frac{P S}{F_{p}}}\right)=F\left(1-H c t_{m}\right)\left(1-e^{-\frac{P S}{F\left(1-H o m^{2}\right)}}\right)
$$


Solving this differential equation gives the solution

$$
C_{e}(t)=\frac{K^{\text {trans }} V_{t}}{V_{e}} \int_{0}^{t} C_{p a}\left(t^{\prime}\right) e^{\frac{-K^{\text {trans }}\left(t-t^{\prime}\right) V_{t}}{V_{e}}} d t^{\prime}
$$

An additional value $E$, the extraction fraction, is the proportion of CA that transfered from $V_{p}$ to $V_{e}$ in one single capillary transit is defined as [6]

$$
E=1-e^{-\frac{P S}{F_{p}}}=1-e^{-\frac{P S}{F(1-H c t m)}}
$$

thus,

$$
K^{\text {trans }}=\frac{E F\left(1-H c t_{m}\right)}{V_{t}} .
$$

In Eq. 2.28, the concentration versus time in the EES is described. To get the total tissue concentration, the vascular compartment must be incorporated. The common ways to deal with this are either to assume the vascular compartment is negligible or to assume that the loss of CA from the vascular system is negligible. The next two sections describe these options.

\section{Small Vascular Fraction Solution}

If the vascular fraction is small, the concentration of the tissue is not largely influenced by the concentration in the vessels. In this case $V_{t} C_{t}$ can be approximated as $V_{e} C_{e}$ [16]. This results in the tissue concentration equation

$$
C_{t}(t)=K^{\text {trans }} \int_{0}^{t} C_{p a}\left(t^{\prime}\right) e^{\frac{-K^{\text {trans }\left(t-t^{\prime}\right) V_{t}}}{V_{e}}} d t^{\prime}
$$

To compare to the discussion in Section 2.2.2, this is equivalent to assuming that the impulse response function is

$$
R(t)=E e^{-\frac{E F(1-H c t) t}{V_{e}}}
$$




\section{Negligible Loss of Vascular Contrast Agent}

If the concentration in the vascular system is not significantly reduced by the diffusion across the capillary wall, vascular contribution to the concentration will be

$$
V_{p} C_{p}(t) \approx V_{p} C_{p a}(t)
$$

If this is the case the equation for $C_{t}(t)$ can be extended to

$$
C_{t}(t)=v_{p} C_{p a}(t)+K^{t r a n s} \int_{0}^{t} C_{p a}\left(t^{\prime}\right) e^{\frac{-K^{t r a n s}\left(t-t^{\prime}\right)}{v_{e}}} d t^{\prime} .
$$

where $v_{p}=V_{p} / V_{t}$ and $v_{e}=V_{e} / V_{t}$.

If the return of the CA to the vascular space is slow, owing to the large volume of the EES, the factor $\exp \left(-K^{\text {trans }}\left(t-t^{\prime}\right) / v_{e}\right)$ is close to one and can be ignored. In this case $K^{\text {trans }}$ can be found through linear regression[17].

$$
C_{t}(t)=v_{p} C_{p a}(t)+K^{\text {trans }} \int_{0}^{t} C_{p a}\left(t^{\prime}\right) d t^{\prime}
$$

This type of analysis works best when applied to the early part of the study, where $C_{p}(t) \gg C_{e}(t)$. At this point $\exp \left(-K^{\text {trans }}\left(t-t^{\prime}\right) / v_{e}\right)$ is approximately 1.

\section{Interpretation of $K^{\text {trans }}$}

Although $K^{\text {trans }}$ is a common perfusion parameter in the literature, there is some confusion about its interpretation. The relation between the volume transfer constant, $K^{\text {trans }}$, and the physiology of the capillary depends on how well the tissue is supplied with blood. In the above model, $K^{\text {trans }}$ is a combination of flow and capillary permeability surface area product. One can imagine two extreme schemes, one where $P S<<F$ and one where $P S>>F$. When $P S<<F$ then $E=P S /(F(1-H c t))$ 
meaning $K^{\text {trans }}=P S / V_{t}$. This represents the case of ample blood flow to the tissue. When $P S>F$ then $E=1$ meaning $K^{\text {trans }}=F(1-H c t) / V_{t}$. This represents the case of insufficient blood supply, this is often referred to as the flow-limited scheme. These two schemes represent very different interpretations of the same parameter thus it is important to consider which scheme reflects the situation under study. In may not be possible to distinguish between the two schemes and often the parameter $K^{\text {trans }}$ is given without further interpretation.

\subsubsection{Adiabatic Approximation to the Tissue Homogeneity Model}

As discussed in the previous section, using measurements which combine permeability with flow can lead to misinterpretation. A large $K^{\text {trans }}$ could mean high flow and low permeability or low flow and high permeability. Thus it is desirable to separate the effects of flow and permeability. The two compartment models disregard the CA's microvascular transit time. This assumption is acceptable if the temporal resolution is greater than the MTT. But once the temporal resolution is comparable with the transit time $(\leq 2.3 \mathrm{~s})$ a model which considers the CA's microvascular transit time can be used to extract permeability and flow independently [18].

St. Lawrence and Lee [19] applied an adiabatic approximation to the tissue homogeneity model which allows for a closed form solution in time space, given certain assumptions. The approximation derives from the assumption that $C_{e}(t)$ changes slowly compared to $C_{p}(t)$. Then for a small time interval, it is assumed that the slow component is steady and the fast component is allowed to evolved. After this the slow component is updated. This assumption is reasonable if the volume of distribution of the slower component is much higher than that of the faster component. This is true in the brain, where the ratio of $V_{e}: V_{p}$ is 20:1 [19]. 
Applying the adiabatic approximation leads to a residue function as follows (see [19] for full derivation);

$$
\begin{gathered}
R(t)=1 \quad \text { if } 0 \leq t<T_{c} \\
R(t)=E e^{E F_{p}\left(t-T_{c}\right) / V_{e}} \quad \text { if } t \geq T_{c}
\end{gathered}
$$

where $T_{c}=V_{p} / F_{p}$ is the transit time through the capillary.

The adiabatic approximation leads to a residue function consisting of two phases. The first phase is referred to by [19] as the vascular phase. During this phase, a fraction of the CA, $E$, is extracted into the interstitial fluid. At a time $t=T_{c}$ the CA present in the vascular compartment exits the tissue. For the remaining time, referred to as the parenchymal tissue phase by [19], the remaining CA $(1-E)$ leaks back into the vascular compartment and is removed from the tissue.

Inserting Eq. 2.36 and Eq. 2.37 into Eq. 2.12 gives

$$
C_{t}(t)=\frac{F_{p}}{V_{t}} \int_{0}^{T_{c}} C_{p a}\left(t-t^{\prime}\right) \mathrm{d} t^{\prime}+\frac{E F_{p}}{V_{t}} \int_{T_{c}}^{t} C_{p a}\left(t^{\prime}\right) e^{\frac{-E F_{p}\left(t-T_{c}-t^{\prime}\right)}{V_{e}}} \mathrm{~d} t^{\prime} .
$$

If a negligibly small $T_{c}$ is assumed, the equation simplifies to

$$
C_{t}(t)=\frac{F_{p}}{V_{t}} T_{c} C_{p a}(t)+\frac{E F_{p}}{V_{t}} \int_{0}^{t} C_{p a}\left(t^{\prime}\right) e^{\frac{-E F_{p}\left(t-T_{c}-t^{\prime}\right)}{V_{e}}} \mathrm{~d} t^{\prime}
$$

which is equivalent to Eq. 2.34 derived in Section 2.2.4.

\subsection{MR Signal Generation}

Magnetic resonance imaging is a safe, non-invasive imaging application of nuclear magnetic resonance (NMR). The NMR signal results from the interaction of a nuclear spin and an external magnetic field. The primary nuclei on which MRI relies are the 
hydrogen nuclei in water and fat in adipose tissue. The signal used to generate the MRI image is induced by manipulating these nuclei using a combination of magnetic fields. Although the NMR phenomenon originates from the quantum mechanical nature of subatomic particles, classical vector models can be applied, in many cases, because MRI deals with the collective behaviour of an ensemble of nuclei.

\subsubsection{Spins in a Magnetic Field}

A fundamental property of nuclei is that those with an odd number of protons or neutrons possess an angular momentum, referred to as spin, $\mathbf{S}[20, \mathrm{p} 26]$. The nuclei themselves are also often referred to as spins in magnetic resonance (MR) literature. The magnetic field produced by the spin is characterized by the nuclear magnetic dipole moment, $\boldsymbol{\mu}$. As described in quantum mechanics, the magnitude of $\boldsymbol{\mu}$ can only take on discrete values. In the absence of an external magnetic field the direction of $\boldsymbol{\mu}$ is random due to thermal motion. However, in the presence of an external magnetic field, $\mathbf{B}_{\mathbf{0}}$, the degeneracy of the spin states is removed. For spin- $-\frac{1}{2}$ particles such as hydrogen nuclei there are two possible orientations for the component of $\boldsymbol{\mu}$ parallel to $\mathbf{B}_{\mathbf{0}}$; aligned with or against the magnetic field. The component of $\boldsymbol{\mu}$ perpendicular to $\mathbf{B}_{\mathbf{0}}$ remains randomly oriented but precesses at an angular frequency, referred to as the Larmor frequency, $\boldsymbol{\omega}_{\mathbf{0}}$. The magnetic moment vector thus traces out a cone where the angle between $\boldsymbol{\mu}$ and $\mathbf{B}_{\mathbf{0}}$ is always $54.74^{\circ}[21, \mathrm{p} 61]$ The Larmor frequency is related to $B_{0}$ via

$$
\omega_{0}=-\gamma \mathbf{B}_{0}
$$

where $\gamma$ is the gyromagnetic ratio. For hydrogen nuclei $\gamma$ is approximately equal to $2.68 \times 10^{8} \mathrm{rad}^{*} \mathrm{~s}^{-1} \mathrm{~T}^{-1}[20, \mathrm{p} 4]$. A group of spins with the same Larmor frequency are collectively referred to as an isochromat. The standard MRI convention of setting $\mathbf{B}_{\mathbf{0}}=B_{0} \hat{z}$ will be used throughout the rest of the thesis. 
MRI does not measure the state of individual spins but rather the bulk properties of many spins. To describe magnetic properties of an ensemble of spins, a macroscopic magnetization vector, $\mathbf{M}$, is defined as the sum of all the magnetic moments in the sample [21, p 64]

$$
\mathbf{M}=\sum_{n=1}^{N_{s}} \boldsymbol{\mu}_{n}
$$

where $N_{s}$ is the number of spins in the sample. In the absence of an external magnetic field, $\mathbf{M}=\mathbf{0}$. In the presence of an external magnetic field, the spins parallel and anti-parallel to $\mathbf{B}_{\mathbf{0}}$ have different energies. For the experimental conditions in the MRI machine there is initially an excess of spins in the lower energy state leading to a net magnetization parallel to the main magnetic field. Thus, in the presence of an external magnetic field, the parallel component of $\mathbf{M}$ in equilibrium, is non-zero and is proportional to the excess of spins parallel to $\mathbf{B}_{\mathbf{0}}$; the perpendicular component remains zero.

\subsubsection{Excitation}

In the external magnetic field, the spin system is in a steady state. To generate a signal, the system is perturbed and the response to this perturbation is monitored [20]. Electromagnetic (EM) radiation with an angular frequency $\omega_{r f}$ is applied to perturb the spin system. If $\omega_{r f}$ matches the Larmor frequency of the spins (i.e. $\omega_{r f}=\omega_{0}$ ), the EM radiation will stimulate transitions between the energy states of the spins; this is referred to as resonance. The net effect of the stimulated transitions is to diminish the excess of protons in the lower energy state thereby reducing the longitudinal (i.e. parallel to $\mathbf{B}_{\mathbf{0}}$ ) component of $\mathbf{M}$. It also establishes a phase coherence amongst the spins, thereby creating a transverse (i.e. perpendicular to $\mathbf{B}_{\mathbf{0}}$ ) component of M. This component will rotate in the transverse plane at the frequency, $\omega_{0}$. The 
electromagnetic radiation required to excite spins in a $1.5 \mathrm{~T}$ field is in the radio frequency (RF) range and is normally only applied for a few milliseconds and thus is referred to as an RF pulse. The RF pulse is produced by a nearby coil, called a transmitter coil. The RF pulse can also be described in terms of its magnetic field $\mathbf{B}_{\mathbf{1}}(t)=B_{1}(t) e^{-i\left(\omega_{r f} t+\phi\right)}$ which oscillates perpendicular to the main magnetic field, $\mathbf{B}_{\mathbf{0}}$. This is a convenient form to use when considering the net results of applying an RF pulse at the resonant frequency of the spins. The net magnetization can be viewed classically as experiencing a torque due to the new net magnetic field $[20, p$ $52]$

$$
\frac{d \mathbf{M}}{d t}=\gamma \mathbf{M} \times\left(\mathbf{B}_{\mathbf{0}}+\mathbf{B}_{\mathbf{1}}\right) .
$$

The result of this torque, when combined with the Larmor precession, is to spiral $\mathbf{M}$ away from $\mathbf{B}_{\mathbf{0}}$. The angle between $\mathbf{B}_{\mathbf{0}}$ and $\mathbf{M}$, referred to as the flip angle, is given by $[21, \mathrm{p} 80]$

$$
\alpha=\int_{0}^{\tau_{p}} \gamma B_{1}(t) \mathrm{d} t
$$

where $\tau_{p}$ is the total time the RF pulse is applied (see Fig. 2.4). A convenient notation used later in the text defines $t=0^{-}$and $t=0^{+}$as the times immediately before and after the RF pulse is applied, respectively. Then the magnetization before and after the pulse can be related by [21, p 83],

$$
\mathbf{M}\left(0^{+}\right)=\mathbf{R}_{i}(\alpha) \mathbf{M}\left(0^{-}\right)
$$

where $\mathbf{R}_{i}(\alpha)$ is the rotation matrix for a rotation about the $\mathrm{i}^{\text {th }}$ axis by angle $\alpha$. 


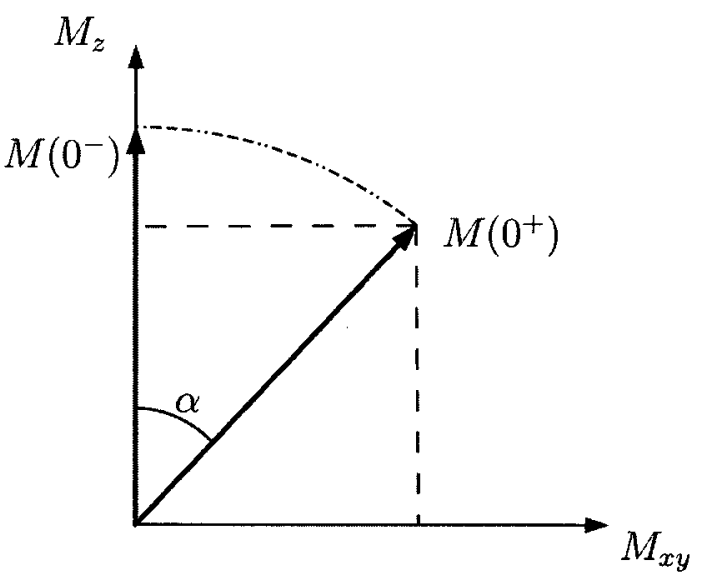

Figure 2.4: View of the magnetization after an RF pulse with flip angle, $\alpha$, in a reference frame rotating at $\omega_{r f}=\omega_{0}$.

\subsubsection{Free Precession and Relaxation}

After the spin system has been perturbed by the RF pulse, a component of $\mathbf{M}$ will rotate in the transverse plane. This is referred to as free precession [21, p 91]. It does not remain in this state indefinitely; it will return to a state of thermal equilibrium.

The rotational and translational motions of the nearby atoms and molecules generate fluctuating magnetic fields owing to their magnetic moments. If the frequencies of the magnetic field fluctuations are at, or near, the Larmor frequency, they can stimulate transitions between the energy states of the spins [20, p 53]. Statistically, the proton spins in the higher energy state are more likely to be stimulated into the lower state and thus the system returns to its equilibrium state with a surplus of spins in the lower energy state. These stimulated transitions do not preserve the phase coherence of the spins and thus the transverse magnetization vector is reduced as well. The net effect is that the longitudinal component of $\mathbf{M}$ grows back to its equilibrium value at a rate characterized by the time constant $T_{1}$, referred to as the longitudinal relaxation time.

In addition to stimulating transitions, the fluctuating fields create local inhomogeneities which change the Larmor frequency of the spins. This, combined with the loss of phase coherence from stimulated transitions, leads to dephasing of the spins 
within a voxel resulting in a loss of magnetization in the transverse plane. The loss of transverse magnetization is characterized by an additional relaxation time, $T_{2}$, referred to as the transverse relaxation time. Because of this additional dephasing mechanism, $T_{2}$ is always less than or equal to $T_{1}$.

A phenomenological description of these processes is summarized in the Bloch equations [20, p 58]:

$$
\frac{d \mathbf{M}}{d t}=\gamma \mathbf{M} \times \mathbf{B}+\frac{1}{T_{1}}\left(M_{0}-M_{z}\right) \hat{z}-\frac{1}{T_{2}} \mathbf{M}_{\mathbf{x y}}
$$

where $M_{0}$ is the magnetization at thermal equilibrium. This applies to liquids and is sufficient for the biological applications of MRI. Adopting the notation presented in the previous section, the magnetization in the longitudinal and transverse planes at some time, $t$, after an $\alpha$ RF pulse are given by [20, p 58]

$$
\begin{gathered}
M_{z}(t)=M_{z}\left(0^{+}\right) e^{-t / T_{1}}+M_{0}\left(1-e^{-t / T_{1}}\right) \\
M_{x y}(t)=M_{x y}\left(0^{+}\right) e^{-t / T_{2}} e^{-i \omega_{0} t}
\end{gathered}
$$

Eq. 2.46 and Eq. 2.47 are represented graphically in Fig. 2.5.

\subsubsection{The Phase of the Magnetization Vector}

Consider the transverse magnetization vector, $M_{x y}$, from the previous section which is rotating at an angular frequency $\omega_{\mathbf{0}}$. It can be seen from Eq. 2.42 that the direction of rotation is in the clockwise direction. If the axes of the transverse plane are defined such that the phase of $M_{x y}$ immediately after the RF pulse is zero, then accumulated phase after a time $t$ is

$$
\phi_{0}(t)=-\int_{0}^{t}\left|\omega_{0}\right| \mathrm{d} \tau=-\gamma B_{0} t
$$




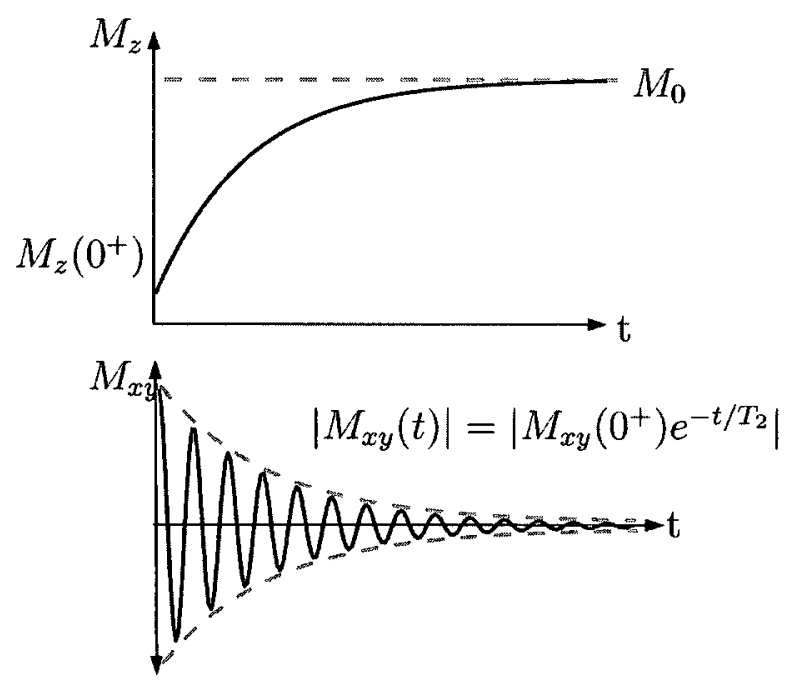

Figure 2.5: $M_{z}$ and $M_{x y}$ relaxation after an RF excitation. See also Eqs. 2.46 and 2.47 .

Next, define a reference frame which rotates at a frequency $\omega_{r f}$ such that if $\boldsymbol{\omega}_{\boldsymbol{r} f}=\boldsymbol{\omega}_{0}$, then $\phi_{0}(t)$ is always zero. This is referred to as the rotating reference frame.

Generally, the spins do not all experience the same magnetic field (i.e. $\mathbf{B} \neq \mathbf{B}_{\mathbf{0}}$ ). Consider the case when all of the spins contributing to the magnetization vector begin with the same phase, but at time $t$ the Larmor frequency varies over the sample owing to a variable magnetic field, $B(\mathbf{r}, t) \hat{z}$. Then the phase accumulation in the rotating reference frame is

$$
\phi(\mathbf{r}, t)=-\left[\int_{0}^{t}|\boldsymbol{\omega}(\mathbf{r}, t)| \mathrm{d} \tau-\int_{0}^{t}\left|\omega_{0}\right| \mathrm{d} \tau\right]=\phi_{0}-\gamma \int_{0}^{t} B(\mathbf{r}, \tau) \mathrm{d} \tau
$$

The individual spins within one voxel may also have different frequencies and thus have different phase accumulations. Here only the phase of the net magnetization vector for the voxel is of interest and thus the above result still applies. The relative dephasing of the spins within a voxel will, however, cause a reduction of the magnitude 
of the vector.

The inhomogeneities in the local magnetic fields present during the excitation result in a distribution of the initial phase of the spins. This leads to a constant initial phase offset, $\phi_{i}(\mathbf{r})$, which is independent of time and varies with location. Thus the phase accumulation at time $t$ in the rotating reference frame is

$$
\phi(\mathbf{r}, t)=\phi_{i}(\mathbf{r})+\phi_{0}-\gamma \int_{0}^{t} B(\mathbf{r}, \tau) \mathrm{d} \tau
$$

In this work only the phase accumulation after the excitation is of interest (see Section 2.6.2). For simplicity $\phi_{i}(\mathbf{r})$ will be ignored in the following discussion of the MRI image, but will be re-introduced in Section 2.6.2.

\subsection{The MRI Image}

\subsubsection{Signal Detection}

The free precession of spins causes a signal through electromagnetic induction in a receiver coil [20, p 99-101]. It is this signal that is used to eventually form the MRI image. As mentioned in Section 2.3.3, the signal decays with time. If there is only one isochromat, then the signal envelope will decay with time constant $T_{2}$, as shown in Fig. 2.5. Often, however, inhomogeneities in the $\mathbf{B}_{0}$ field lead to multiple isochromats and consequently to additional dephasing. This results in a faster decay, characterized by $T_{2}^{*}$. If imperfections in the excitation or detection of the signal are ignored then the received signal will be

$$
S^{\prime}(t)=\int_{o b j e c t} M_{x y}\left(\mathbf{r}, 0^{+}\right) e^{-t / T_{2}^{*}(\mathbf{r})} e^{-i \omega(\mathbf{r}) t} \mathrm{~d} \mathbf{r}
$$


where $M_{x y}\left(\mathbf{r}, 0^{+}\right)$is the transverse magnetization at position $\mathbf{r}$ immediately after the application of an RF pulse. In practice, there are many factors which may alter the signal. For example, imperfect excitation or various factors in the recording of the signal (e.g. amplifier gains) will introduce scaling factors. An effort is made to make the scaling uniform over the whole image volume, but this is not exact. For this reason the data is recorded in arbitrary units and the image is not a direct map of the magnetization.

\subsubsection{Localization}

At this point in the discussion, the signal recorded by the receiver coils would be the sum of the signals from all the spins in the sample, as described in Eq. 2.51. To create an image, the spins must be "localized". This is achieved by applying a magnetic field gradient to produce spatially varying precessional frequencies in the direction of the gradient (e.g. $\boldsymbol{\omega}(x)=-\gamma \mathbf{B}(x)$ ). The frequency of a spin then represents its spatial location along the magnetic field gradient. These gradients can be introduced independently in the $x, y$ and $z$ directions and, since they are typically applied for durations of 1-10 ms, there are referred to as gradient pulses. The variation of frequencies created by the gradient pulses can be used to selectively excite spins at a specific location (called selective-excitation), or to encode the locations of the spins (spatial encoding) [20, p 7-8]. For 2D sequences, a slice selection gradient pulse is applied during the RF pulses such that only the spins in one particular slice are excited. This is then followed by the spatial encoding gradients. In the case of a 3D sequence, the entire volume to be imaged is excited by the RF pulses in the presence of a suitable gradient and then spatial encoding of the MR signal is performed for

all three dimensions. In this section, the gradient field will be explained in more detail, followed by a description of its application to selective excitation and spatial encoding. 


\section{Magnetic Field Gradients}

Define the gradient field, $\mathbf{B}_{\mathbf{G}, \mathbf{z}}$, as a magnetic field whose component parallel to $\mathbf{B}_{\mathbf{0}}=B_{0} \hat{z}$ varies along a specific direction, referred to as the gradient direction:

$$
\mathbf{B}_{\mathbf{G}, \mathbf{z}}=B_{G, z} \hat{z}=\left(G_{x} x+G_{y} y+G_{z} z\right) \hat{z}=(\mathbf{G} \cdot \mathbf{r}) \hat{z}
$$

where $\mathbf{G}$ is the gradient in units of magnetic field per unit distance $\left(\mathrm{Tm}^{-1}\right)$ and where the direction of $\mathbf{G}$ is the gradient direction. This field is added to the external magnetic field to give the total applied field:

$$
\mathbf{B}=\left(B_{0}+\mathbf{G} \cdot \mathbf{r}\right) \hat{z}
$$

The presence of a magnetic field gradient means that spins at difference locations experience different magnetic fields and thus accumulate different phases. In the rotating reference frame the phase accumulation, $\phi(\mathbf{r}, t)$, at any point during the application of a gradient pulse of duration $\tau$ is given by

$$
\phi(\mathbf{r}, t)=-\gamma \int_{0}^{t}(\mathbf{G} \cdot \mathbf{r}) \mathrm{d} t^{\prime}, \quad 0 \leq t \leq \tau .
$$

As time passes the spins dephase and the MR signal quickly decays to zero (see Fig. 2.6). If this gradient pulse is followed by a gradient pulse of the same duration but opposite polarity along the same direction then the phase accumulation at any point during the second gradient pulse is given by

$$
\phi(\mathbf{r}, t)=-\gamma \int_{0}^{\tau}(\mathbf{G} \cdot \mathbf{r}) \mathrm{d} t^{\prime}-\gamma \int_{\tau}^{t}(-\mathbf{G} \cdot \mathbf{r}) \mathrm{d} t^{\prime}, \quad \tau \leq t \leq 2 \tau
$$

From this equation it can be seen that $\phi(\mathbf{r}, 2 \tau)=0$ for this pair of gradient pulses. 


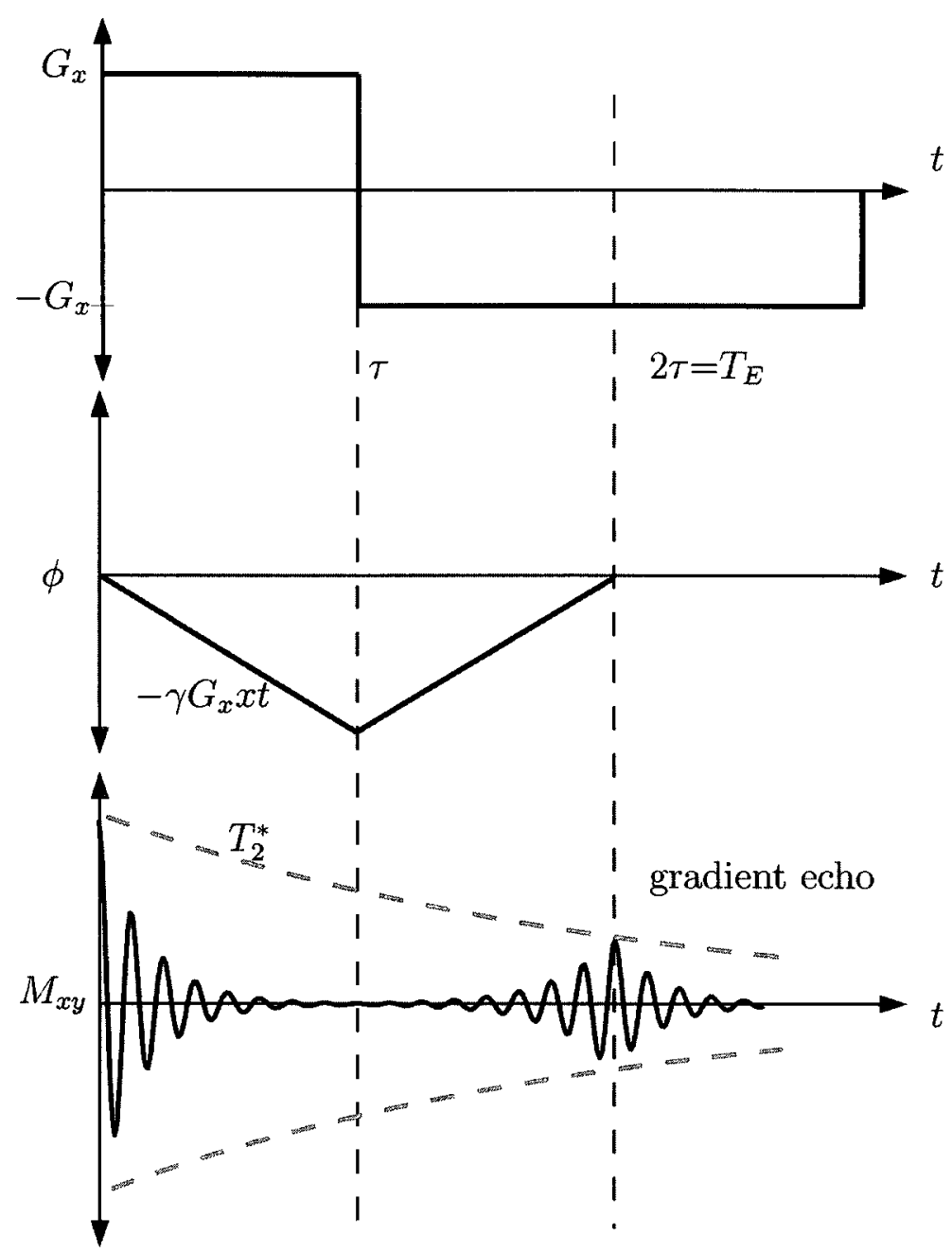

Figure 2.6: Schematic of gradient echo formation.

This means that the spins will all be in phase at a time $2 \tau$ and the transverse magnetization is at a local maximum. This is referred to as a gradient echo, see Fig. 2.6. Note that any dephasing caused by $\mathbf{B}_{\mathbf{0}}$ inhomogeneities will not be rephased. The peak of the echo occurs when the areas under the two gradient lobes are equal. Further echoes can be produced in the same manner as long as there is sufficient signal. These are referred to as multi-echo sequences. In this work single and double gradient echo sequences are used.

\section{Slice Selection}

During the RF pulse, a magnetic field gradient is applied causing the spins at different positions along the slice select direction to have different precessional frequencies. Only spins at locations matching the range of RF rotational frequencies contained in 
the RF pulse will be excited. This means that the measured signal will originate only from the desired slice location. The excited spins will become dephased with respect to one another because of the slice selection gradient and thus an additional gradient must be applied after the RF pulse is finished to rephase the spins.

The distribution of flip angles, and hence the magnitude of the transverse magnetization, along the slice select direction is called the slice profile. It is dependent on the shape of the envelope of the applied RF pulse, $B_{1}(t)$. To get a perfect rectangular slice profile, $B_{1}(t)$ must equal a sinc function of infinite extent. In practice, the RF pulse must be short in duration; thus, only a few lobes are included, leading to non-uniform excitation along the slice select direction.

\section{Spatial Encoding}

Next the spins within the slice must be localized. To do this a gradient perpendicular to the slice select gradient is applied while the signal is being recorded. This is called the readout gradient or frequency encode gradient, $\mathbf{G}_{\mathrm{fe}}$. It causes spins at positions along the gradient direction, $\mathbf{r}_{\mathbf{f}}$, to have different precessional frequencies which are related to the locations of the spins. In the rotating reference frame, the angular frequency along the direction of the frequency encode gradient is given by

$$
\boldsymbol{\omega}\left(\mathbf{r}_{\mathbf{f e}}\right)=-\gamma \mathbf{G}_{\mathbf{f e}} \cdot \mathbf{r}_{\mathbf{f e}} \hat{z}
$$

Localization along the final dimension is achieved by applying an additional gradient orthogonal to the slice select and readout gradients. It is called the phase encode gradient, $\mathbf{G}_{\mathbf{p e}}$. This gradient is applied when there is no excitation or recording of the data so it has no effect on the measured frequency of the spins. It will, however, cause a phase shift in the transverse plane, similar to that discussed in the slice select 
section:

$$
\phi\left(\mathbf{r}_{\mathbf{p e}}\right)=-\gamma \mathbf{G}_{\mathbf{p e}} \cdot \mathbf{r}_{\mathbf{p e}} \tau_{p e}
$$

where $\tau_{p e}$ is the length of time the phase encode gradient is applied. For a 3D acquisition this phase encoding process is performed in two orthogonal directions.

The whole pulse sequence (excite-prepare-read) is repeated using different phase shifts (by varying the gradient amplitude) to build up spatial information in the phase encode direction. The time between successive RF excitations is defined as the repetition time or $T_{R}$.

\subsubsection{Image Formation}

The sum of all the spatial encoding leads to the following signal equation for a 3D acquisition,

$$
S^{\prime}\left(k_{x}, k_{y}, k_{z}\right)=\int_{-\infty}^{\infty} \int_{-\infty}^{\infty} \int_{-\infty}^{\infty} S(x, y, z) e^{-i 2 \pi\left(k_{x} x+k_{y} y+k_{z} z\right)} \mathrm{d} x \mathrm{~d} y \mathrm{~d} z
$$

where

$$
2 \pi k_{i}=\gamma \int_{0}^{t} G_{i} \mathrm{~d} \tau
$$

and $S(x, y, z)$ is a complex function which is proportional to the complex transverse magnetization vector. For a $2 \mathrm{D}$ acquisition the $\mathrm{z}$ direction is not included. By applying an inverse Fourier transform, $S(x, y, z)$ can be retrieved from the recorded signal, $S^{\prime}\left(k_{x}, k_{y}, k_{z}\right)$. The final result is an image in the form of a matrix of complex numbers. The magnitude and phase of the complex signal are

$$
|S|=\sqrt{S_{R}^{2}+S_{I}^{2}}
$$


and

$$
\phi=\tan ^{-1}\left(\frac{S_{I}}{S_{R}}\right)
$$

where $S_{R}$ and $S_{I}$ are the real and the imaginary components of the signal, respectively.

\subsection{Noise Properties of the MRI Signal}

The MRI signal from each coil is recorded using two independent, perpendicular channels the responses from which are recorded as complex numbers. Sources of random error in the MRI signal include electrical resistance in the receiver coil, dielectric and inductive losses in the sample and noise in the preamplifier electronics and RF transmitter [22]. The random electronic noise implies that the signal in each channel follows a Gaussian probability distribution. When the signal is transformed from k-space to image space, via a Fourier transform, the signal retains its Gaussian probability distribution because the Fourier transform is a linear operation.

In image space, the probability density function of the signal from each channel, $\rho_{i}$, is

$$
\rho_{i}=\frac{1}{\sqrt{2 \pi \sigma_{i}^{2}}} \exp \left(-\frac{\left(S_{i}-A_{i}\right)^{2}}{2 \sigma_{i}^{2}}\right)
$$

where $i$ is $\mathrm{R}$ or $\mathrm{I}$, representing the real or imaginary component of the signal, $S_{i}$ is the noisy signal intensity, $A_{i}$ is the true magnitude of the signal (i.e. the signal that would be obtained in the absence of noise) and $\sigma_{i}^{2}$ is the variance of the noise. It is assumed that the variance of the noise is the same for each channel (i.e. $\sigma_{I}^{2}=\sigma_{R}^{2}$ ) and that the correlation coefficient between the noise in the real and imaginary channels is zero [22]. These are common assumptions in MRI. This variance is defined as $\sigma_{g}^{2}$. A very common way of describing the amount of random noise in the images is to 
define what is called the signal-to-noise ratio (SNR). It is given as

$$
S N R=\frac{A}{\sigma_{g}}
$$

where $A=\sqrt{A_{I}^{2}+A_{R}^{2}}$.

\subsubsection{Noise in Magnitude Images}

For most MRI applications the magnitude of the complex data is used to form the images. It is often assumed that the distribution of the magnitude signal is also Gaussian but this is not rigorously correct since the calculation of the magnitude of a vector is not a linear operation. After taking the magnitude, the probability density function instead follows a Rician distribution [23]. The result of this is to make the signal appears larger than it is since there is a bias toward higher numbers. This effect is only significant at low SNR levels since in the limit of high $\operatorname{SNR}\left(A / \sigma_{g}>3\right)$ the magnitude signal distribution approximates a Gaussian distribution. In the case of sufficient SNR the standard deviation of the magnitude is

$$
\sigma_{|S|}=\sigma_{g}
$$

In the limit of no signal $\left(A / \sigma_{g}=0\right)$ the magnitude distribution becomes a Rayleigh distribution:

$$
\rho_{A=0}=\frac{|S|}{\sigma^{2}} \exp \left(-\frac{|S|^{2}}{2 \sigma^{2}}\right) .
$$

The first moment and variance of $\rho_{A=0}$ are

$$
<|S|_{A=0}>=\sqrt{\sigma_{g}^{2} \frac{\pi}{2}}
$$


and

$$
\sigma_{|S|_{A=0}}^{2}=\sigma_{g}^{2}(2-\pi / 2)
$$

\subsubsection{Noise in Phase Images}

The standard deviation of the phase can be found by applying propagation of random error to Eq. 2.61 as presented by Conturo and Smith [22]. This derivation involves making the assumption that the magnitude data follows a Gaussian distribution (i.e. sufficient SNR).

From the propagation of random error, variance in the phase is

$$
\sigma_{\phi}^{2}=\left(\frac{\partial \phi}{\partial S_{R}}\right)^{2} \sigma_{S_{R}}^{2}+\left(\frac{\partial \phi}{\partial S_{I}}\right)^{2} \sigma_{S_{I}}^{2}+2 \rho\left(\frac{\partial \phi}{\partial S_{R}}\right)\left(\frac{\partial \phi}{\partial S_{I}}\right) \sigma_{S_{R}} \sigma_{S_{I}}
$$

where $\rho$ is the coefficient of correlation between the real and imaginary noise. Applying the derivative chain rule, the partial derivatives are

$$
\left(\frac{\partial \phi}{\partial S_{R}}\right)=-\cos ^{2} \phi \frac{S_{I}}{S_{R}^{2}}
$$

and

$$
\left(\frac{\partial \phi}{\partial S_{I}}\right)=\frac{\cos ^{2} \phi}{S_{R}}
$$

respectively. Apply the assumption that $\rho=0$ and $\sigma_{S_{R}}^{2}=\sigma_{S_{I}}^{2}=\sigma_{g}$ as described above to Eq. 2.68 and insert Eqs. 2.69 and 2.70 to get,

$$
\sigma_{\phi}^{2}=\sigma_{g}^{2}\left(\frac{\cos ^{4} \phi\left(S_{R}^{2}+S_{I}^{2}\right)}{S_{R}^{4}}\right)
$$

Taking the square root and simplifying gives,

$$
\sigma_{\phi}=\frac{\sigma_{g}}{|S|}
$$




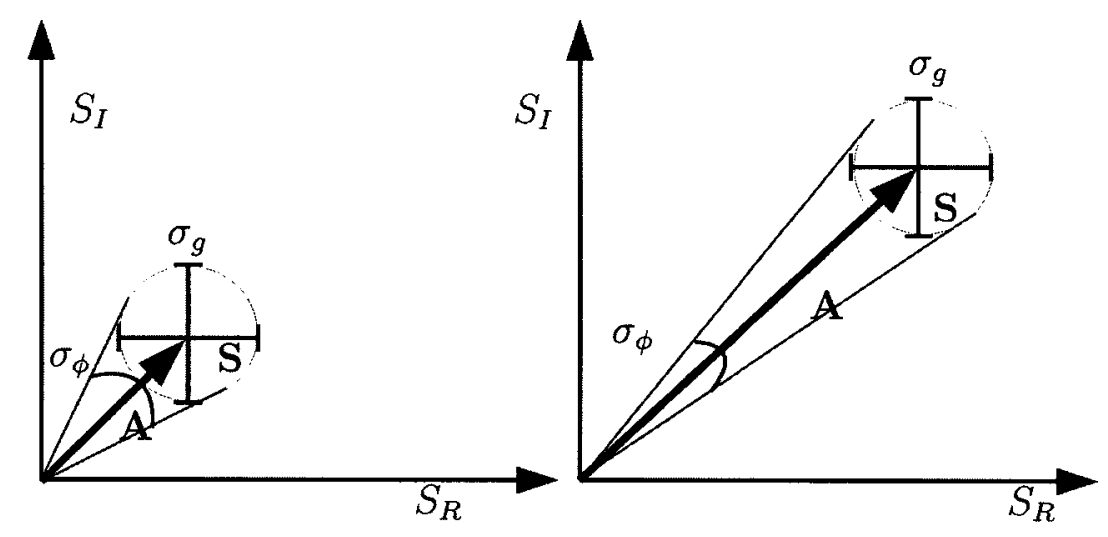

Figure 2.7: Schematic of the effect of $|S|$ on $\sigma_{\phi}$. The arrow indicates $A$ the true MR signal without noise and the error bars represent $\sigma_{g}$ the error in $S_{R}$ and $S_{I}$. From this one can imagine a distribution of signal vectors, $\mathbf{S}$, represented by the shaded gray area. By comparing the two diagrams it can be seen that the diagram on the right with the longer vector $\mathbf{A}$ has less of a range of angles and thus $\sigma_{\phi}$ is lower.

where $\sigma_{\phi}$ is the standard deviation of the phase and $|S|$ is the magnitude of the complex signal as defined in Eq. 2.60. Thus there is less noise in $\phi$ when $|S|$ is larger, as shown schematically in Fig. 2.7.

It is important to note that, for the images acquired in this work, increases in [CA] result in increases in the signal magnitude. Since $|S|$ is in the denominator of Eq. 2.72, the random error on the phase of the MRI signal is reduced when the [CA] is higher.

\subsection{The Spoiled Gradient Echo Sequence}

There are many different combinations of RF pulses and magnetic field gradient pulses which can be used to achieve a wide variety of image contrast. Each set of RF pulses and gradient pulses is called an MRI pulse sequence. The spoiled gradient 


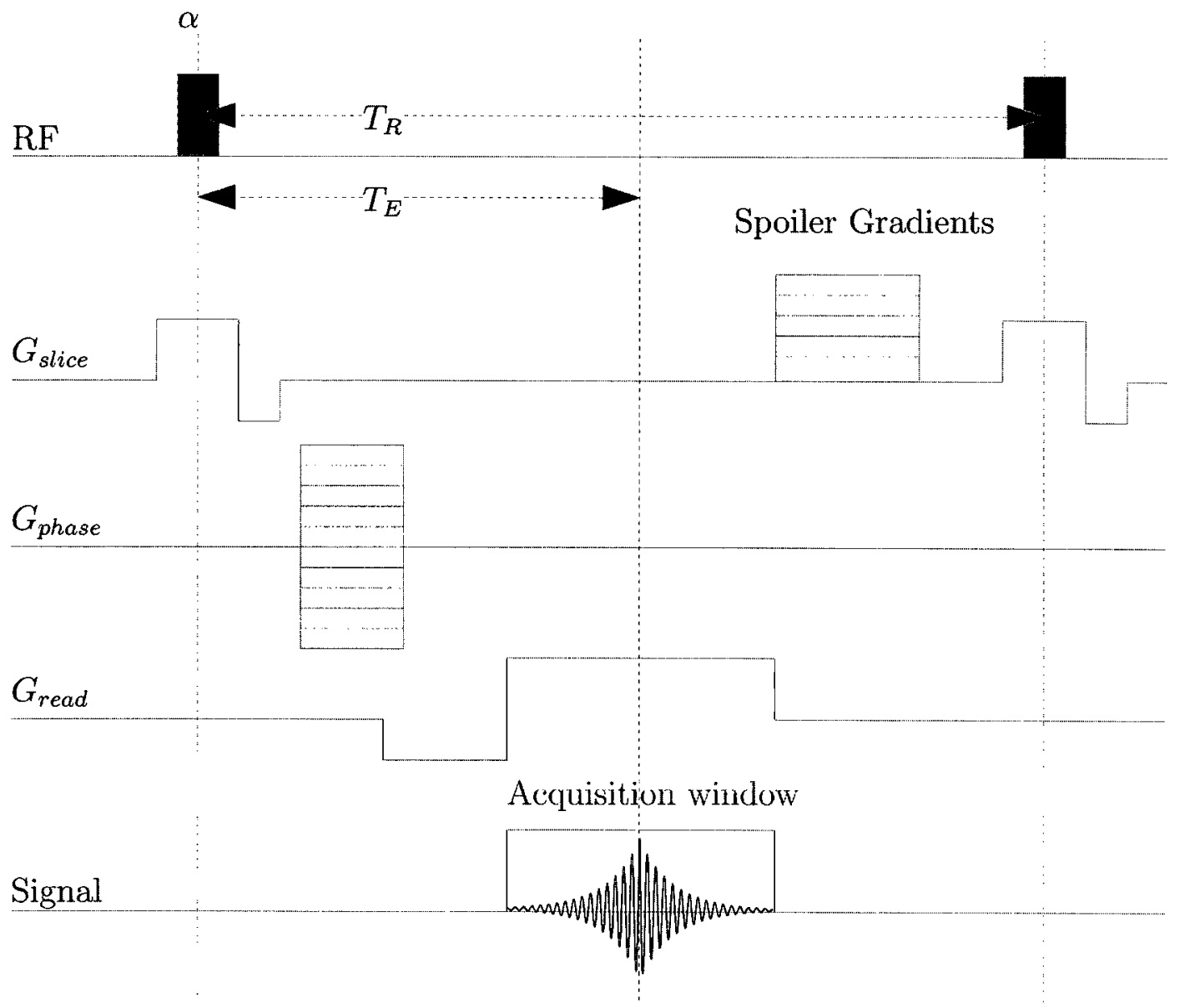

Figure 2.8: A sequence diagram for a 2D spoiled gradient echo (SPGR) sequence. 
echo (SPGR) sequence is used extensively throughout this work. The following is a derivation of the signal strength equation for the magnitude (Section 2.6.1) and the phase (Section 2.6.2) of an SPGR sequence [21, p 226]. This information will be used in the conversion of signal to [CA] described in Section 2.8.

\subsubsection{Signal Magnitude}

As described in the preceding sections, in MRI the spin system is excited by a series of periodic RF pulses separated by a time, $T_{R}$. After a sufficient number of pulses the system reaches dynamic equilibrium. The magnetization after the $n^{\text {th }}$ RF pulse is

$$
\begin{aligned}
& M_{z}^{n}\left(0^{+}\right)=M_{z}^{n}\left(0^{-}\right) \cos \alpha \\
& M_{x y}^{n}\left(0^{+}\right)=M_{z}^{n}\left(0^{-}\right) \sin \alpha
\end{aligned}
$$

where $M_{z}^{n}\left(0^{-}\right)$is the longitudinal magnetization immediately before the $n^{\text {th }}$ RF pulse and $M_{z}^{n}\left(0^{+}\right)$and $M_{x y}^{n}\left(0^{+}\right)$are the longitudinal and transverse magnetizations immediately after the $n^{\text {th }}$ RF pulse, respectively. From the solution to the Bloch equation it can be shown that for a time $T_{R}$ after the $n^{\text {th }}$ RF pulse, the magnetization is given by

$$
M_{z}^{n}\left(T_{R}\right)=M_{z}^{n}\left(0^{-}\right) \cos \alpha e^{-T_{R} / T_{1}}+M_{0}\left(1-e^{-T_{R} / T_{1}}\right)
$$

and

$$
M_{x y}^{n}\left(T_{R}\right)=e^{-T_{R} / T_{2}} M_{z}^{n}\left(0^{-}\right) \sin \alpha
$$

With an SPGR sequence the residual transverse magnetization $\left(M_{x y}^{n}\right)$, after the data collection, is destroyed by either a spoiler gradient or RF spoiling, or both. Spoiler gradients cause dephasing of the signal. RF spoiling consists of changing the phase of the RF pulse for each excitation so that signal does not accumulate. In either case, the goal is to completely destroy all of the transverse magnetization after 
each data acquisition while not affecting the longitudinal magnetization.

At this point the next RF pulse is applied. Assuming that perfect spoiling is achieved (i.e. $M_{x y}^{n}\left(0^{-}\right)=M_{x y}^{n+1}\left(0^{-}\right)=0$ ), the longitudinal magnetization after this pulse is

$$
M_{z}^{n+1}\left(0^{-}\right)=M_{z}^{n}\left(0^{-}\right) \cos \alpha e^{-T_{R} / T_{1}}+M_{0}\left(1-e^{-T_{R} / T_{1}}\right)
$$

A steady state is reached when $M_{z}^{n}\left(0^{-}\right)=M_{z}^{n+1}\left(0^{-}\right)$. Let the steady state longitudinal magnetization be $M_{z}^{s s}\left(0^{-}\right)$. Then rearranging Eq. 2.75 and substituting

$$
E_{1}=e^{-T_{R} / T_{1}}
$$

gives

$$
M_{z}^{s s}\left(0^{-}\right)=\frac{M_{0}\left(1-E_{1}\right)}{1-\cos \alpha E_{1}}
$$

The signal arising from the gradient echo at an echo time, $T_{E}$ is

$$
S=M_{z}^{s s}\left(0^{-}\right) \sin \alpha e^{-T_{E} / T_{2}^{*}}
$$

Combining Eqs. 2.79 and 2.80 gives the complete magnitude signal strength equation,

$$
S\left(M_{0}, T_{R}, \alpha, T_{E}, T_{1}, T_{2}^{*}\right)=\frac{M_{0}\left(1-e^{-T_{R} / T_{1}}\right)}{1-\cos \alpha e^{-T_{R} / T_{1}}} \sin \alpha e^{-T_{E} / T_{2}^{*}}
$$

A convenient short form used later is to define a term $M_{0}^{\prime}$

$$
M_{0}^{\prime}=M_{0} e^{-T_{E} / T_{2}^{*}}
$$

which gives

$$
S=M_{0}^{\prime} \sin \alpha \frac{1-E_{1}}{1-\cos \alpha E_{1}}
$$


The maximum signal occurs when

$$
\cos \alpha_{E}=E_{1}
$$

where $\alpha_{E}$ is referred to as the Ernst angle [20, p 457]. The Ernst angle increases as the ratio $T_{R} / T_{1}$ increases.

\subsubsection{Signal Phase}

The signal phase accumulates from the time of the RF pulse until the acquisition of the signal. In the case of a gradient echo sequence, signal acquisition occurs at the echo time, $T_{E}$. Consider a series of gradient echo scans each with the same $T_{E}$, as would be the case in a dynamic series acquisition. Assume the magnetic field at position $\mathbf{r}$ is quasistatic during the scan and only changes between scans. The phase accumulation, $\Delta \phi(\mathbf{r}, t)$ between $t_{0}$, the time of the baseline image and a later scan at time $t$ is then given by

$$
\Delta \phi(\mathbf{r}, t)=-\gamma T_{E}\left(B(\mathbf{r}, t)-B\left(\mathbf{r}, t_{0}\right)\right)
$$

If the $T_{E}$ and the change in the magnetic field are both small enough that $-\pi<$ $\Delta \phi(\mathbf{r}, t)<\pi$, then there is a unique one-to-one mapping of phase to magnetic field.

\subsection{Materials in a Magnetic Field}

The static field experienced by the protons within tissue differs slightly from the external applied field. This is because the material generates its own magnetic field when placed in an external magnetic field. Section 2.7.1 defines magnetic susceptibility and its relation to the magnetic field. Section 2.7 .2 describes the exact solution 
for the magnetic field inside and outside of an infinite cylinder where the cylinder and its surroundings have different susceptibilities. Section 2.7.3 describes a computational method for determining the magnetic field shift caused by an arbitrary magnetic susceptibility distribution.

\subsubsection{Magnetic Susceptibility}

Consider a material in an external magnetic field, $\mathbf{B}_{\mathbf{0}}$. The material will modify the magnetic field experienced at a given location both inside and outside of the material due to magnetic fields induced by the material internally. Thus, the actual magnetic field, $\mathbf{B}(\mathbf{r})$, experienced at a location $\mathbf{r}$, will be a combination of the applied magnetic field, $\mathbf{B}_{\mathbf{0}}$, and a contribution from the material. This total field will be the magnetic field which determines the precessional frequency of the measured spins. An auxiliary field, $\mathbf{H}\left(\mathrm{Am}^{-1}\right)$ is introduced to isolate the magnetic field caused by external sources from the effect of the material. The value of $\mathbf{H}$ is constant whether there is a material present or not. $\mathbf{H}$ is defined as $[20, \mathrm{p} 474]$

$$
\mathbf{H}=\mathbf{B} / \mu_{\mathbf{0}}-\mathbf{M}
$$

where $\mu_{0}$ is the permeability of free space $\left(4 \pi \times 10^{-7} \mathrm{TmA}^{-1}\right)$ and $\mathrm{M}$ is the magnetization $\left(\mathrm{Am}^{-1}\right)$ of the material. The magnetization is defined as the volume density of the net magnetic dipole moment. Typically $\mathbf{M}$ is weak compared to the applied field and disappears when the field is removed (except in the case of ferromagnetism which is not relevant to the present discussion). Note that the $\mathbf{M}$ described here is not the same as the magnetization vector described in Section 2.3.1, which was the sum of nuclear magnetic moments. If the magnetization, $\mathbf{M}$ is linearly related to $\mathbf{H}$ through a constant, the material is said to be linear [20, p 474]. This constant is 
Table 2.1: Classification of materials based on magnetic susceptibility.

\begin{tabular}{cc}
\hline Material Type & Magnetic Susceptibility \\
\hline diamagnetic & $\chi<0$ \\
non-magnetic & $\chi=0$ \\
paramagnetic & $\chi>0$ \\
ferromagnetic & $\chi>>1$ \\
water & $-1 \times 10^{-5}[25]$ \\
\hline
\end{tabular}

called the magnetic susceptibility of the material, $\chi$, where

$$
\mathbf{M}=\chi \mathbf{H}
$$

The behaviour of materials in a magnetic field is grouped into several classifications based on their magnetic susceptibility [20, p 748](see Table 2.1). Water and human tissues are diamagnetic [20, p 747] and MRI CAs generally consist of low concentrations of paramagnetic molecules in aqueous solutions [24]. For all cases relevant to this thesis the condition $|\chi|<<1$ will be valid.

By substituting Eq. 2.87 into Eq. 2.86, the magnetic field in the presence of the material with magnetization $\mathbf{M}$ is

$$
\mathbf{B}=\frac{1+\chi}{\chi} \mu_{0} \mathbf{M}
$$

\subsubsection{Analytic Solution for a Cylinder}

The magnetic field shift caused by the presence of an infinite cylinder can be calculated analytically. First the case of an infinite cylinder of a continuous medium in free space will be considered. Then the atomic or molecular nature of the material is accounted for and incorporated into the expression. Finally, the magnetic field shift caused by the presence of a cylinder in a surrounding material of a different magnetic 


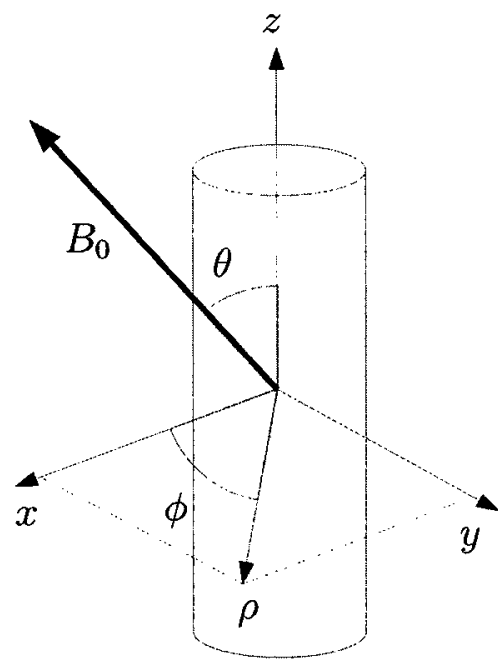

Figure 2.9: Coordinate system definition for cylinder in a magnetic field.

susceptibility is considered. This is done to facilitate comparison with the numerical methods presented in Section 2.7.3.

Consider an infinite cylinder of radius $a$ with uniform magnetic susceptibility, $\chi$. Next, define a coordinate system as in Fig. 2.9 where $\rho=\sqrt{\rho_{x}^{2}+\rho_{y}^{2}}$ and $\tan \phi=\rho_{y} / \rho_{x}$. Let the external magnetic field, $\mathbf{B}_{\mathbf{0}}$, be uniform and at an angle $\theta$ with respect to the cylinder.

Haacke et al. have shown that the magnetic fields inside and outside of an infinite cylinder in the general case where $\mathbf{B}_{0}=B_{0}(\sin \theta \hat{x}+\cos \theta \hat{z})$, have the following forms $[20, \mathrm{p} 753]$

$$
\begin{gathered}
\mathbf{B}_{\text {out }}(\rho, \phi)=B_{0} \cos \theta \hat{z}+B_{0} \sin \theta\left(\hat{x}+\frac{\chi / 2}{1+\chi / 2}\left(\frac{a}{\rho}\right)^{2}(\cos 2 \phi \hat{x}+\sin 2 \phi \hat{y})\right) \\
\mathbf{B}_{\text {in }}(\rho, \phi)=B_{0} \cos \theta(1+\chi) \hat{z}+B_{0} \sin \theta \frac{1+\chi}{1+\chi / 2} \hat{x} .
\end{gathered}
$$


In this discussion, only the shift in magnetic field along the original magnetic field direction is of interest, that is $\Delta B_{\| B_{0}}=\Delta \mathbf{B} \cdot \hat{B}_{0}$ where $\Delta \mathbf{B}=\mathbf{B}-\mathbf{B}_{\mathbf{0}}$. The change in magnetic field along the axis of the magnetic field due to the presence of an infinite cylinder, in the limit of $|\chi|<<1$, is [20, p 753],

$$
\begin{gathered}
\Delta B_{\| B_{0}}(\rho>a) \approx \frac{\chi}{2}\left(\frac{a}{\rho}\right)^{2} \cos 2 \phi \sin ^{2} \theta B_{0} \\
\Delta B_{\| B_{0}}(\rho<a) \approx \frac{\chi}{2}\left(1+\cos ^{2} \theta\right) B_{0} .
\end{gathered}
$$

The situation considered thus far is that of a continuous medium. In reality, spins and their immediate neighbours cannot be considered to be a continuous distribution of magnetic field sources. There is a necessary correction which considers the cancellation of the induced fields of nearby neighbours. This is referred to as the Lorentz sphere correction [20, p 753]. The local magnetic field experienced by the spins is a combination of the field calculated for a continuous medium and the reduction in field cause by the internal structure of the material:

$$
\Delta B_{\| B_{0}, \text { local }}=\Delta B_{\| B_{0}, \text { continuous }}+\Delta B_{\| B_{0}, \text { internal }}
$$

$\Delta B_{|| B_{0}, \text { internal }}$ can be calculated as the change in magnetic field caused by the removal of a sphere of magnetic susceptibility $\chi$, which is much smaller than the radius of the vessel but large compared to the intermolecular distances within the vessel [20, p 754]. The change in magnetic field parallel to the external field due to the presence of a sphere can be calculated in a way similar to the method used to calculated the effect of a cylinder [20, p 750] and is given as

$$
\Delta B_{\| B_{0}}=\frac{2}{3+\chi} B_{0} \chi
$$


If $|\chi|<<1$ then Eq. 2.94 simplifies to

$$
\Delta B_{\| B_{0}}=\frac{2}{3} \chi B_{0}=-\Delta B_{\| B_{0}, \text { internal }}
$$

Combining this with Eq. 2.92 gives

$$
\begin{aligned}
\Delta B_{\| B_{0}, l o c a l}(\rho<a) & \approx \frac{\chi}{2}\left(1+\cos ^{2} \theta\right) B_{0}-\frac{2}{3} \chi B_{0} \\
& \approx \frac{\chi B_{0}}{6}\left(3 \cos ^{2} \theta-1\right) .
\end{aligned}
$$

In a vacuum, there is no internal structure, implying that Eq. 2.91 does not require a Lorentz correction. Equations 2.91 and 2.97 are the expressions for $\Delta B_{|| B_{0}}$ needed to calculate the corresponding change in Larmor frequency and the resulting phase shift in the MR signal for a cylinder in a vacuum. For clinically relevant situations, the vessel is not in a vacuum; it is surrounded by tissue. In the presence of a background material with susceptibility, $\chi_{e}$, the Lorentz correction is needed for the background as well. Again, in a way similar to the calculation for a cylinder, the change in the magnetic field due to the presence of an infinite medium with magnetic susceptibility, $\chi_{e}$ is

$$
\Delta B_{\| B_{0}, \text { background }}=\left(\left(1+\chi_{e}\right) \mathbf{B}_{0}-\mathbf{B}_{\mathbf{0}}\right) \cdot \hat{B}_{0}-2 / 3 \chi_{e} B_{0}=\frac{1}{3} \chi_{e} B_{0} .
$$

In this situation the $\chi$ in Eq. 2.91 and Eq. 2.97 becomes [20, p 755]

$$
\Delta \chi=\chi_{i}-\chi_{e}
$$

where $\chi_{i}$ is the magnetic susceptibility inside the vessel. Thus, the shift in magnetic field, $B_{M}(\rho, \phi)$, in the presence of an infinite cylinder with magnetic susceptibility, 
$\chi_{i}$, and background tissue with magnetic susceptibility, $\chi_{e}$, at a location $(\rho, \phi)$, is

$$
B_{M}(\rho, \phi)=\Delta B_{\| B_{0}, \text { cylinder }}+\Delta B_{\| B_{0}, \text { background }}
$$

$B_{M}(\rho, \phi)$ is used here instead of $\Delta B$ to facilitate comparison with Section 2.7.3. This gives the average magnetic field shift in the direction of the main magnetic field caused by the presence of a material; it does not necessarily correspond to the magnetic field experienced by the spins locally. Substituting the expressions for a cylinder (Eqs. 2.91 and 2.97) and the background (Eq. 2.98) gives

$$
\begin{gathered}
B_{M}(\rho>a)=\frac{\Delta \chi}{2}\left(\frac{a}{\rho}\right)^{2} \sin ^{2} \theta \cos 2 \phi B_{0}+\frac{1}{3} \chi_{e} B_{0} \\
B_{M}(\rho<a)=\frac{\Delta \chi}{6}\left(3 \cos ^{2} \theta-1\right) B_{0}+\frac{1}{3} \chi_{e} B_{0}
\end{gathered}
$$

\subsubsection{Dipole Approximation Method}

The following method, proposed by Marques and Bowtell [25], provides a computational method to calculate the shift in the magnetic field caused by an object in the magnetic field. Unlike the analytic method presented above, this method can be applied to an arbitrary magnetic susceptibility distribution. Details of the implementation of this method are presented in Section 5.2.1

Given a material with a known distribution of magnetic susceptibility, $\chi(\mathbf{r})$, and a uniform external magnetic field, $\mathbf{B}_{\mathbf{0}}$, the goal is to calculate the magnetic field shift, $B_{M}(\mathbf{r})$. In this approach, the magnetic field shift is calculated by summing up the magnetic dipolar fields generated by each element in the magnetization distribution $[25]:$ 


$$
\mathbf{B}_{d}(\mathbf{r})=\frac{\mu_{0}}{4 \pi} \int \frac{1}{\left|\mathbf{r}-\mathbf{r}^{\prime}\right|^{3}}\left(3 \frac{\mathbf{M}\left(\mathbf{r}^{\prime}\right) \cdot\left(\mathbf{r}-\mathbf{r}^{\prime}\right)}{\left|\mathbf{r}-\mathbf{r}^{\prime}\right|^{2}}\left(\mathbf{r}-\mathbf{r}^{\prime}\right)-\mathbf{M}\left(\mathbf{r}^{\prime}\right)\right) d^{3} \mathbf{r}^{\prime}
$$

for $\mathbf{r}-\mathbf{r}^{\prime} \neq 0$, where $\mathbf{r}$ is the location of measurement of the field and $\mathbf{r}^{\prime}$ is the location of the element of the magnetization distribution.

In the Fourier domain this equation has the form [25]

$$
\tilde{\mathbf{B}}_{d}(\mathbf{k})=\frac{\mu_{0}}{3} \frac{3 \cos ^{2} \beta-1}{2}\left(\tilde{\mathbf{M}}(\mathbf{k})-3 \tilde{M}_{z}(\mathbf{k}) \hat{z}\right)
$$

where

$$
\cos ^{2} \beta=\frac{\left(k_{z} \cos \theta-k_{y} \sin \theta\right)^{2}}{k_{x}^{2}+k_{y}^{2}+k_{z}^{2}}
$$

and $\theta$ is the angle between the $z$ axis of the volume and the magnetic field in the $y z$ plane. As in the previous section, the magnetic field in the $x y$ plane is not relevant and only the $z$ component is computed. This leads to the following expression

$$
\tilde{\mathbf{B}}_{d, z}(\mathbf{k})=-\frac{\mu_{0}}{3}\left(3 \cos ^{2} \beta-1\right) \tilde{M}_{z}(\mathbf{k})
$$

$\mathbf{B}_{\mathbf{d}}(\mathbf{r})$ (comparable to Eqs. 2.102 and 2.101) is found by taking the inverse Fourier transform of Eq. 2.106. Note that this includes the effect of the Lorentz sphere inherently in the calculation.

\subsection{Method for Dynamic Contrast-Enhanced MRI}

The first step in the DCE-MRI procedure is to measure the [CA] as a function of time for every pixel in the image. These concentration-time curves, depending on their location in the image, correspond to either the tissue response function (TRF) or the arterial input function (AIF), which are both needed as inputs for the perfusion 
models discussed in Section 2.2.1. During a dynamic series acquisition the same image protocol is applied at each time point; the only change in contrast is due to the change in the [CA] over time. Both the magnitude and the phase of the MR signal are affected by the $[\mathrm{CA}]$, meaning that both can be exploited to estimate concentration. Magnitude methods perform better for the low concentrations found in tissue whereas, for the higher concentrations found in the major blood vessels, both magnitude and phase methods can be applied. Within the magnitude methods there are different protocols designed for different situations including whether the sequence is $2 \mathrm{D}$ or $3 \mathrm{D}$ and the SNR.

\subsubsection{The MRI Contrast Agent and its Effect on the NMR Tissue Properties}

The most common MRI contrast agents are low molecular weight gadolinium chelates. Gadolinium is toxic, therefore it must be chelated in a stable complex to make it safe for use as a $\mathrm{CA}$ in human subjects. These chelates are small enough that the CA is able to pass from the capillaries into the extracellular fluid, although it does not cross an intact blood brain barrier or the cellular membrane. One commonly used complex is diethylenetriamine pentaacetic acid (DTPA). This CA (Gd-DTPA) is sold

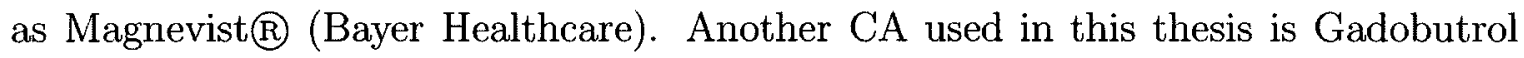
(Gd-DO3A-butrol), sold as Gadovist $\AA$ (Bayer Healthcare).

In contrast with other imaging techniques, such as computed tomography (CT) or positron emission tomography (PET), the tracer used in MRI is not detected directly. As explained in the theory section, the MRI machine measures the NMR properties

of the water and fat protons only. The $\mathrm{Gd}$ in the CAs provide image contrast by altering the magnetic environment of the nearby protons. 
When a water molecule is near the paramagnetic Gd atom, electron-nuclear dipolar interactions occur with the unpaired outer shell electrons. These unpaired electrons produce a large electronic magnetic moment which is much greater than the nuclear magnetic moment produced by the protons. This leads to a significant change in the local magnetic environment of the water molecule. Because of the constant motion of the CA molecules, the magnetic moments they generate fluctuate. The frequency of these fluctuations covers a wide range, including the right frequency to stimulate transitions between the energy states of the spins, shortening $T_{1}$ and $T_{2}$. Additionally, the large magnetic moment of the CA introduces magnetic field inhomogeneities over long ranges, leading to additional $T_{2}$ relaxation. As a result, the effect of the CA on $T_{2}$ extends over a large range whereas the effect on $T_{1}$ is local. These changes are observed as a variation in the signal intensity in the image which is then related to the $[\mathrm{CA}]$.

When a CA molecule is present in the tissue, the water molecules diffuse into and out of the region of influence of the CA due to their Brownian motion. They do this rather quickly; in fact, the average nearby water molecule will interact with the $\mathrm{CA}$ thousands to millions of times before diffusing out of the region of influence of the CA. For a $1 \mathrm{mM}$ solution of $\mathrm{CA}$ it takes an average of $3 \mu \mathrm{s}$ for the water to diffuse from one CA molecule to another [26]. In a single compartment the spins experience an apparent $T_{1}$ which is a combination of the initial $T_{1}$ of the tissue and the additional relaxation caused by the CA. The relationship between the relaxation times and the $[\mathrm{CA}]$ are

$$
\begin{aligned}
& \frac{1}{T_{1}}=\frac{1}{T_{1,0}}+\lambda_{1} C, \\
& \frac{1}{T_{2}}=\frac{1}{T_{2,0}}+\lambda_{2} C,
\end{aligned}
$$

where $\lambda_{1}$ and $\lambda_{2}$ are relaxivity and $C$ is the [CA] in mM. $T_{1,0}$ and $T_{2,0}$ are the relaxation 
times in the absence of the CA. Magnevist $\left(\mathrm{B}\right.$ ) has a $T_{1}$ relaxivity of $3.6 \mathrm{mM}^{-1} \mathrm{~s}^{-1}$ and a $T_{2}$ relaxivity of $5.5 \mathrm{mM}^{-1} \mathrm{~s}^{-1}$ in water at $1.5 \mathrm{~T}$ at $20^{\circ} \mathrm{C}$ [27]. In human plasma at $1.5 \mathrm{~T}$ and $37^{\circ} \mathrm{C}$, a $T_{1}$ relaxivity of $3.9 \pm 0.2 \mathrm{mM}^{-1} \mathrm{~s}^{-1}$ and a $T_{2}$ relaxivity of $5.3 \pm 0.2$ $\mathrm{mM}^{-1} \mathrm{~s}^{-1}$ were found [28]. These are comparable values suggesting that the effects of temperature and plasma versus water are small. Gadovist@ has a $T_{1}$ relaxivity of $4.7 \pm 0.2 \mathrm{mM}^{-1} \mathrm{~s}^{-1}$ and a $T_{2}$ relaxivity of $6.8 \pm 0.2 \mathrm{mM}^{-1} \mathrm{~s}^{-1}$ under the same conditions $[28]$.

\subsubsection{The Effect of the Contrast Agent on Signal Phase}

Next consider what happens to the phase of the MR signal as a CA passes through the body. From Eq. 2.85 it is clear that anything which causes a variation in the magnetic field over time will also affect the MR signal phase throughout the dynamic series. As discussed in Section 2.7.1, the presence of a material with a non-zero magnetic susceptibility affects the magnetic field experienced by the spins inside the material and in the immediate vicinity of the material. The magnetic susceptibility inside the blood vessels is altered by the addition of a CA. The magnetic susceptibility, $\chi_{i}$, inside the blood vessel is given by [29]

$$
\chi_{i}=\chi_{0}+\chi_{m} C
$$

where $\chi_{m}$ is the molar magnetic susceptibility, $\chi_{0}$ is the magnetic susceptibility of the tissue and $C$ is the concentration of $\mathrm{Gd}$ atoms in $\mathrm{M}$. The molar magnetic susceptibility for $\mathrm{Gd}$ is $3.4 \times 10^{-4} \mathrm{M}^{-1}$ in SI units [30]. The resulting magnetic field can be written in terms of the external magnetic field strength plus the magnetic field induced by the material.

$$
\mathbf{B}(\mathbf{r}, t)=\mathbf{B}_{\mathbf{0}}+\mathbf{B}_{\mathbf{M}}(\mathbf{r}, t)
$$


where $\mathbf{B}_{\mathbf{M}}(\mathbf{r}, t)$ is defined in Eq. 2.88 .

The observed magnetic field changes depend not only on the magnetic susceptibility change caused by the CA but also by the geometry and orientation of the compartment containing the $\mathrm{CA}$ with respect to $\mathbf{B}_{\mathbf{0}}$. If an analytic solution relating $\chi(\mathbf{r})$ to $\mathbf{B}(\mathbf{r})$ is known, an equation relating $[\mathrm{CA}]$ and phase change can be derived [30]. For more complex geometries, a magnetic field map is needed. The most common case encountered in this work is for blood vessels which are relatively straight (e.g. the superior sagittal sinus, see Fig. 6.2).

Recall the case of an infinite straight vessel at an angle $\theta$ with respect to $\mathbf{B}_{\mathbf{0}}$ discussed in Section 2.7.2. In the DCE experiment, the vessel begins at time $t_{0}$, with no CA. The magnetic field induced by the presence of an infinite material, with magnetic susceptibility $\chi_{0}$, along the direction of $\mathbf{B}_{\mathbf{0}}$ is (see Eq. 2.98)

$$
B_{M}\left(\mathbf{r}, t_{0}\right)=\frac{1}{3} \chi_{0} B_{0}
$$

After a time, $t$ the $[\mathrm{CA}], C(t)$, increases and thus the magnetic susceptibility inside the vessel changes. The magnetic field inside the vessel induced by the presence of the tissue containing the $\mathrm{CA}$ along the direction of $\mathbf{B}_{0}$ is

$$
B_{M}(\mathbf{r}, t)=\frac{B_{0} C(t) \chi_{m}}{3} \frac{\left(3 \cos ^{2} \theta-1\right)}{2}+\frac{1}{3} \chi_{0} B_{0} .
$$

The phase difference inside the vessel, measured between these time points, can be found from Eq. 2.85 and is

$$
\Delta \phi(t)=\gamma T_{E}\left(\frac{B_{0} C(t) \chi_{m}}{3} \frac{\left(3 \cos ^{2} \theta-1\right)}{2}\right)
$$

Note that the negative sign has been removed in the previous equation, and thus an increase in [CA] leads to a positive $\Delta \phi(t)$. This is done to be consistent with 


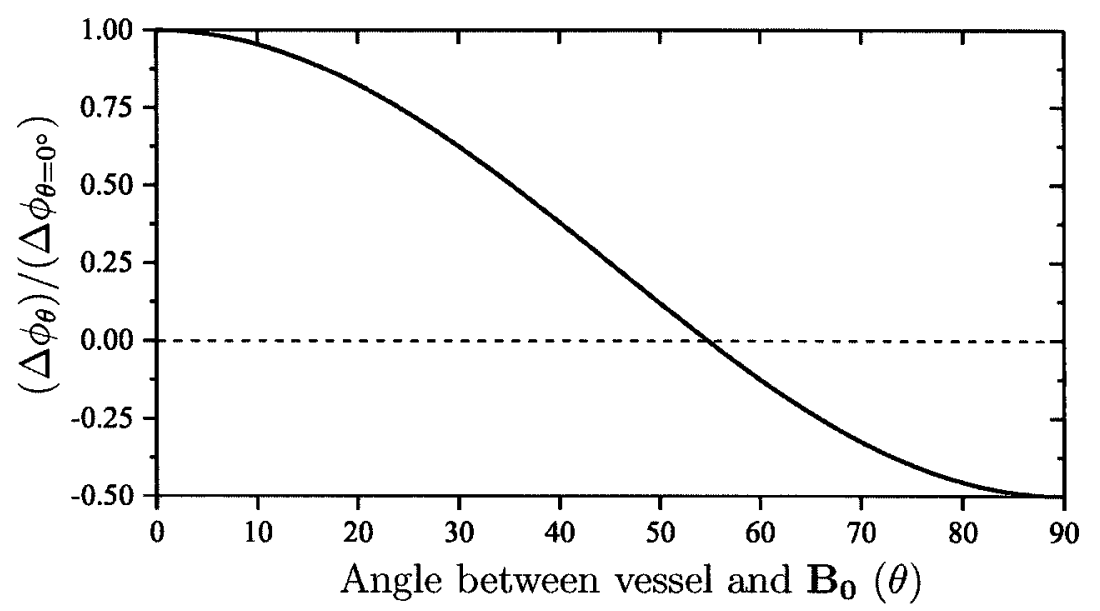

Figure 2.10: Phase difference for a infinite straight vessel at an angle $\theta$ with respect to $\mathbf{B}_{\mathbf{0}}$ divided by the phase difference at $\theta=0^{\circ}$, for the same change in [CA].

the coordinate system convention of the MRI scanner and with what is commonly reported in the literature. This convention will be used for the remainder of the thesis.

In the case of a straight vessel, $C(t)$ can be calculated from the phase accumulation as a function of time if $\theta$ is known:

$$
C(t)=\frac{3 \Delta \phi(t)}{\gamma T_{E} B_{0} \chi_{m}} \frac{2}{\left(3 \cos ^{2} \theta-1\right)}=\frac{3 \Delta \phi(t)}{\gamma T_{E} B_{0} \chi_{m}} g(\theta)
$$

where $g(\theta)$ is a geometry factor equal to a maximum value of 1 for $\theta=0$.

Although the geometry factor is well defined at all angles, not all angles provide the same phase difference SNR, as can be seen from Fig. 2.10. Vessels which are approximately parallel to the external magnetic field will provide the most precise measurements. Since there is also error associated with the measurement of the angle, the most accurate results can be achieved when the derivative of the geometry factor with respect to $\theta$ is the smallest.

Next consider the case of a general vessel geometry. As discussed in Section 2.7.3, if the magnetic susceptibility map is known, the magnetic field shift, $B_{M}(\mathbf{r}, t)$, can 
be calculated. In the DCE experiment, the magnetic susceptibility is not known at each time point since it depends on the $[\mathrm{CA}]$ which is not known. However, for an arbitrary vessel geometry it can be shown that $B_{M}(\mathbf{r}, t)$ is a linear function of $C(t)$ for the case when $\chi<<1$ [29]. Consequently, the difference in phase accumulation, $\Delta \phi$, between the $[\mathrm{CA}]=0$ and $[\mathrm{CA}]=C(t)$ situations is also linear. It will be assumed that this linear relationship can be expressed as

$$
C(t)=k \Delta \phi(t) g(\mathbf{r})
$$

where $k$ is a constant and $g(\mathbf{r})$ is a generalized geometry factor. $k$ can easily be evaluated by realizing that, since Eq. 2.115 should be valid for all vessel geometries, it must reduce to Eq. 2.114 for an infinitely long cylinder. This gives:

$$
C(t)=\frac{3 \Delta \phi(t)}{\gamma T_{E} B_{0} \chi_{m}} g(\mathbf{r})
$$

The generalized geometry factor, $g(\mathbf{r})$, is, in general, difficult to calculate but an efficient method for performing this calculation is proposed in Section 5.2.

\subsubsection{Effect of Contrast Agent on the Spoiled Gradient Echo Sequence}

As discussed in Section 2.8.1, the relationship between [CA] and the relaxation times is essentially linear. The pharmacokinetic models require high temporal resolution so the dynamic series must be acquired quickly. There is not sufficient time to take an independent $T_{1}$ or $T_{2}$ measurement at each time point. Instead signal differences relative to the pre $\mathrm{CA}$ injection time points are related to changes in $[\mathrm{CA}]$ for each time point in the dynamic series. 
All dynamic series collected for this thesis utilize the spoiled gradient echo sequence discussed in Section 2.6. For this sequence the presence of the CA causes a shortening of $T_{1}$, which means areas of increased [CA] appear brighter in a $T_{1}$-weighted image.

For the SPGR sequence, the relationship between the relaxation times and the magnitude of the signal is not linear, leading to a non-linear relationship between signal magnitude and [CA]. Consider the SPGR signal equation given in Eq. 2.83. For constant $T_{R}, T_{E}$, and $\alpha$, the equation can be rewritten as a constant $M_{0}$ times a function of [CA], $f(C)$ :

$$
S=M_{0} f(C)
$$

The function $f(C)$ is plotted in Fig. 2.11 for a typical $T_{1}$-weighted 3D protocol and several $T_{1,0}$ values typical of those found in tissue. The extensive curvature is due in part to the $T_{2}^{*}$ effects, which are present even in a $T_{1}$-weighted protocol because of the non-zero echo time. It can be seen from Fig. 2.11 that the value of $T_{1,0}$ also has an effect on the relationship between signal magnitude and [CA]. For this reason, if quantitative measurements of $[\mathrm{CA}]$ are desired, then $T_{1,0}$ must be measured.

The presence of the CA also affects the phase of the SPGR signal. The induced phase shift inside a straight vessel that is at an angle $\theta$ to the main magnetic field is given by Eq. 2.113. Consider the effect of the CA on the signal magnitude and phase simultaneously. Setting $\theta=0$ and using the same protocol as in Fig. 2.11 leads to a signal vector which rotates in the complex plane and increases in magnitude as [CA] increases (Fig. 2.12).

The signal change depicted in Fig. 2.12 is for the case when the signal is from a single homogeneous population of spins (isochromat). In reality, the signal from a single voxel may come from different isochromats. This can occur when the object does not completely fill the voxel in question, such as for the outer voxels in Fig. 


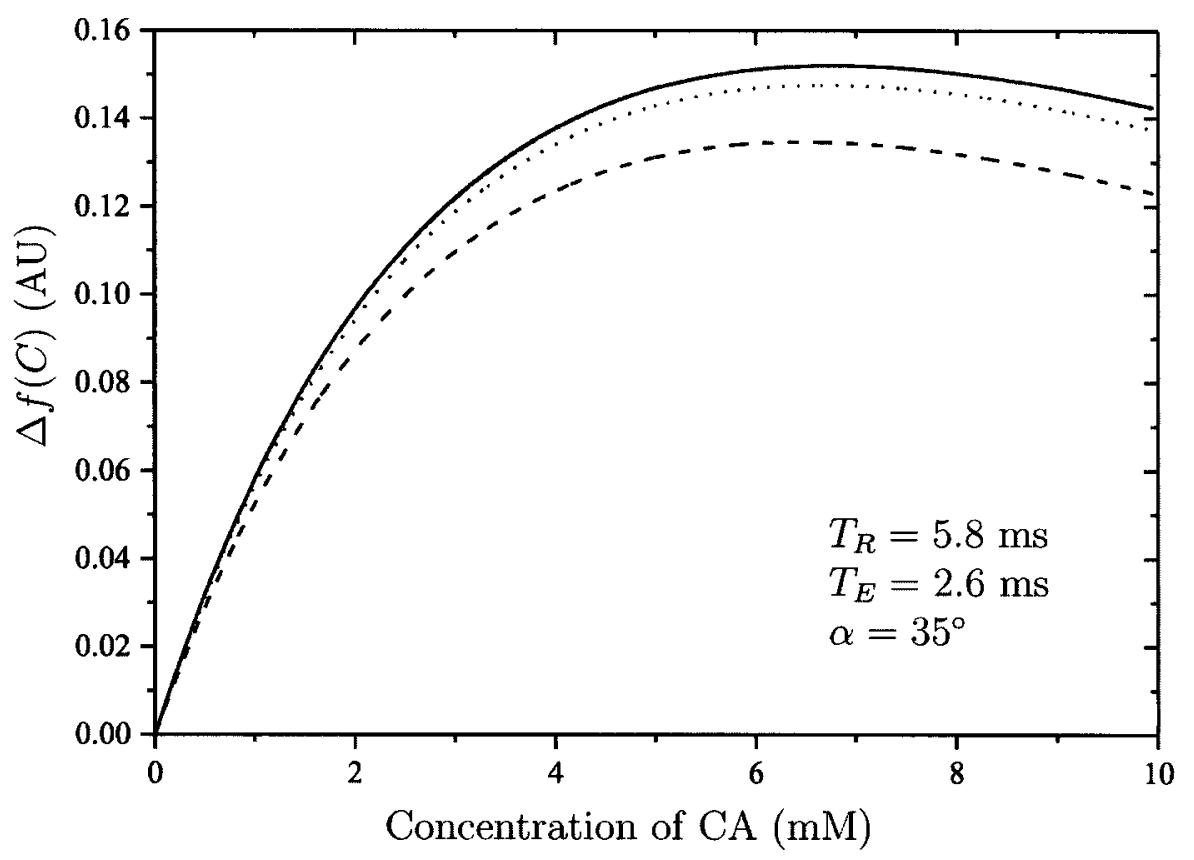

Figure 2.11: Graph showing the non-linear relationship between signal magnitude from an SPGR sequence (A.U.) and [CA] for a typical 3D protocol and several precontrast $T_{1}$ values typical of tissue. The curves for $T_{1,0}=1500 \mathrm{~ms}, T_{1,0}=1000 \mathrm{~ms}$ and $T_{1,0}=500 \mathrm{~ms}$ shown as solid, dotted and dashed lines, respectively.

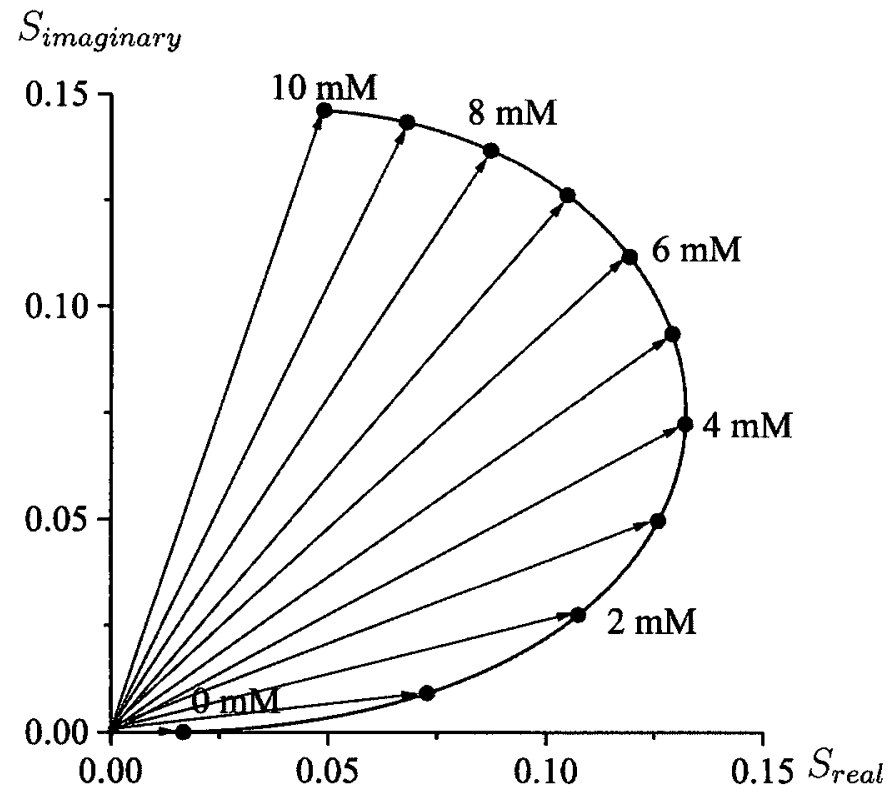

Figure 2.12: Graph showing the relationship between the complex signal from an SPGR sequence and $[\mathrm{CA}]$. 


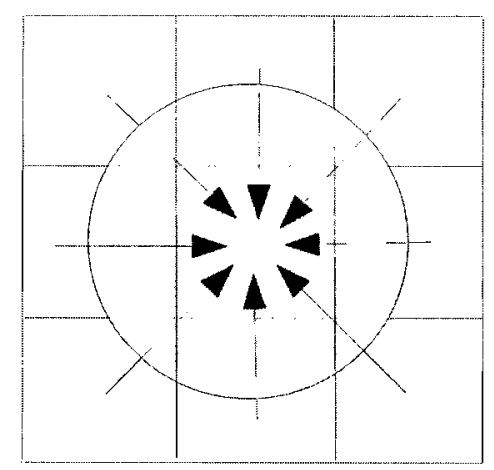

Figure 2.13: Diagram showing an example object with a pixel grid to illustrate the concept of partial volumes and signal leakage.

2.13. It can also come from spins outside the voxel of interest due to the point spread function; this means, for example, that there can be an influence from a signal external to a blood vessel even if the voxel is completely inside the vessel, as is shown schematically for the central pixel in Fig. 2.13. Whatever the cause, the measured signal becomes the sum of the contributing complex signals

$$
S_{v o x e l}=f_{v} S_{v} e^{i \phi_{v}}+f_{e} S_{e} e^{i \phi_{e}}
$$

where $f_{3}$ is the fraction of the total signal coming from inside the vessel are fo the the fraction of the signal coming from outside the vessel due to either partial volumes or signal leakage from the point spread function. $S_{v}$ and $S_{e}$ are the signal magnitudes from inside and outside the vessel and $\phi_{v}$ and $\phi_{e}$ are the corresponding phases.

Consider the case where the $\mathrm{CA}$ is present inside a vessel and [CA] changes as a function of time as shown in Fig. 2.14 a). If it is assumed that there is no enhancement outside the vessel, then the signal originating from outside the vessel does not change. Consider the effect of an external signal on the measured phase. Fig. 2.14 b) shows $f_{v} S_{v} e^{i \phi_{v}}$, the signal from inside the vessel and $S_{v o x e l}$, the total signal which has been shifted because of the extra contribution, both on the complex plane. Fig. $2.14 \mathrm{c}$ ) 
and d) then show the measured phase versus time and resulting [CA] estimated from that phase, with and without signal corruption from the external signal. It becomes apparent that it is the baseline phase which is most susceptible to the corruption by external signals because its signal strength is lower. The error in the concentration, however, appears in the peak because the concentration is taken by computing the phase accumulation from the baseline. It is important to recognize that an external signal does not necessarily lead to an overestimation of [CA]. It can also lead to an underestimation of [CA] as depicted in Fig. 2.15.

The severity of this effect varies, depending on the relative size and direction of the two vectors in Eq. 2.118. Simulations were performed by Dr. Greg Cron in our lab [31] to assess the potential error caused by an external contribution for the brain perfusion protocol applied in Chapter 6, for an SNR level of 15:1. The area under the curve (AUC) was used to assess the error. It was found that for $f_{e} S_{e}<f_{v} S_{v}$ the deviation in the AUC was less than $10 \%$ when the two vectors are parallel or anti-parallel. For cases were the two vectors are perpendicular, the error is larger. For example, an external contribution with a magnitude of $15 \%$ compared to the baseline will cause an error of $30 \%$. Fortunately, for the tissues studied in the research reported in this thesis the surrounding signal and the baseline tend to have similar phases. Once the enhancement begins, the phase of the vessel will change but this has a minimal effect on the concentration results since the magnitude is also increasing. An external signal which is aligned with the baseline and is also enhancing will not affect the

phase results as the external vector will rotate in the same direction as the internal vector.

\subsection{4 $T_{1}$ Map Using the Variable Flip Angle Method}

For the SPGR sequence the conversion from signal magnitude to [CA] requires knowledge of the tissue $T_{1}$ before contrast injection, $T_{1,0}$. A time-efficient way to determine 

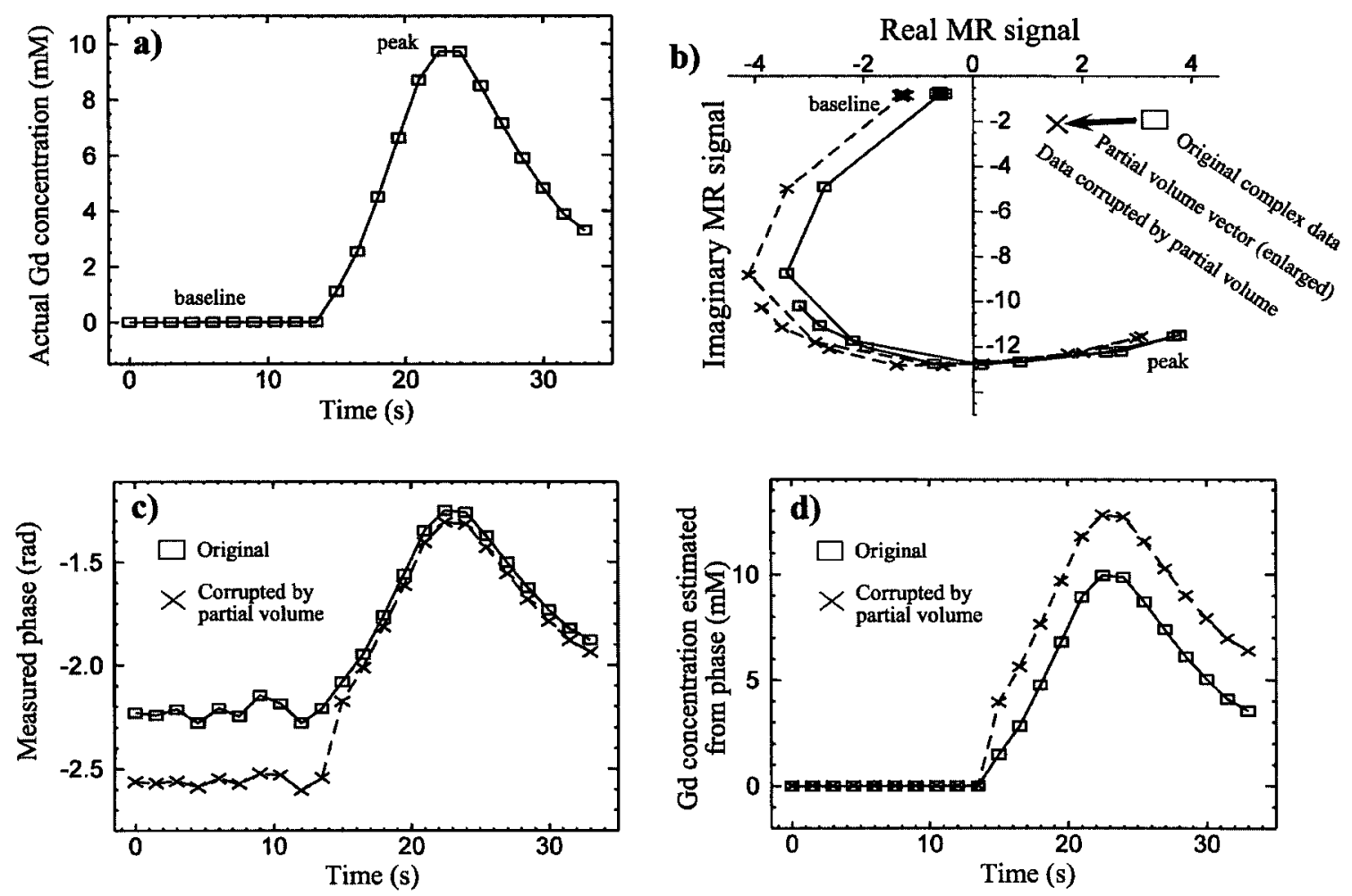

Figure 2.14: Phase signal corruption leading to an overestimation of [CA]. a) A typical input function. b) The complex MR signal with and without corruption from an external signal. c) The measured phase accumulation with and without signal corruption. d) The measured [CA] with and without signal corruption. Note that the points in b) evolve as a function of time from the points labelled "baseline" to the points labelled "peak" and then back towards the baseline again. 

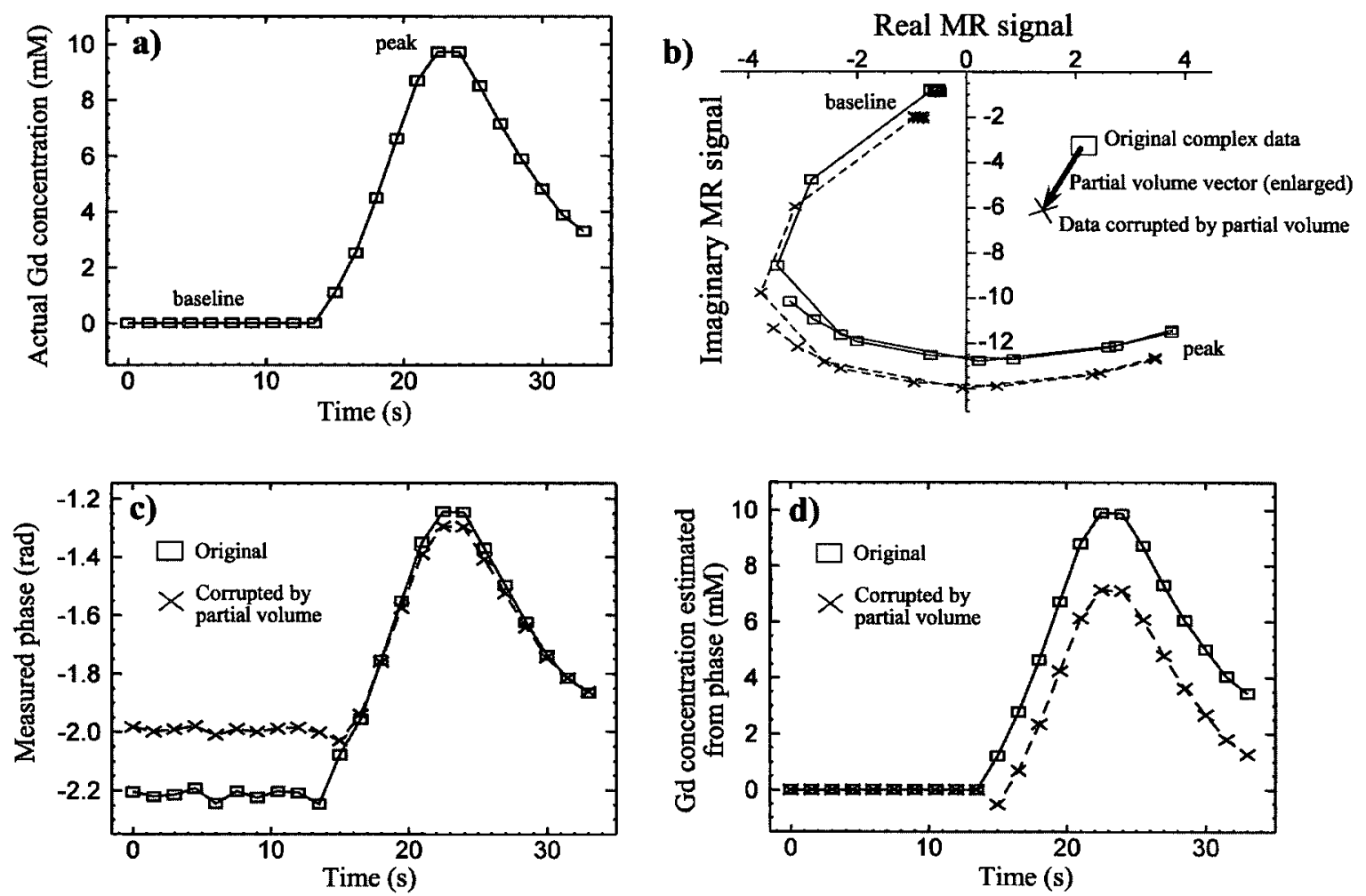

Figure 2.15: Phase signal corruption leading to an underestimation of [CA]. a) A typical input function. b) The complex MR signal with and without corruption from an external signal. c) The measured phase accumulation with and without signal corruption. d) The measured [CA] with and without signal corruption. Note that the points in b) evolve as a function of time from the points labelled "baseline" to the points labelled "peak" and then back towards the baseline again. 
this is to use the variable flip angle (VFA) method proposed by Deoni et. al. [32]. The data acquisition consists of a series of SPGR images, each with a different flip angle, $\alpha_{i}$, with all other imaging parameters held constant. Using the SPGR signal equation, under the assumption of perfect spoiling, Eq. 2.83, the following rearrangement can be made:

$$
\frac{S_{\alpha_{i}}}{\sin \alpha_{i}}=\frac{S_{\alpha_{i}}}{\tan \alpha_{i}} E_{1}+M_{0}^{\prime}\left(1-E_{1}\right) .
$$

Applying a linear regression to $S_{\alpha_{i}} / \sin \alpha_{i}$ versus $S_{\alpha_{i}} / \tan \alpha_{i}$ gives a slope of $m=E_{1}$ and an intercept of $b=M_{0}^{\prime}\left(1-E_{1}\right)$. Therefore, $T_{1,0}$ can be calculated from

$$
T_{1,0}=-\frac{T_{R}}{\ln (m)}
$$

Once $T_{1,0}$ is known, the magnitude images from the dynamic series can be converted into maps of [CA] as described in the next section.

\subsubsection{Using the Magnitude of the MRI Signal to Measure Contrast Agent Concentration}

A simple view of the SPGR signal equation suggests that, if $T_{1,0}$ is known, then the [CA] versus time can be calculated from direct manipulation of the SPGR equation. This method, referred to here as the $T_{1}$ pre method, generally works well in the case of $3 \mathrm{D}$ acquisitions and is the standard method found in the literature. $2 \mathrm{D}$ acquisitions, which are sometimes used because of their superior time resolution, suffer from inaccuracies in the flip angle and a direct application of the SPGR signal equation, in this case, results in errors. A discussion of this issue and a method for dealing with it, referred to here as the Bookend method are presented below. 


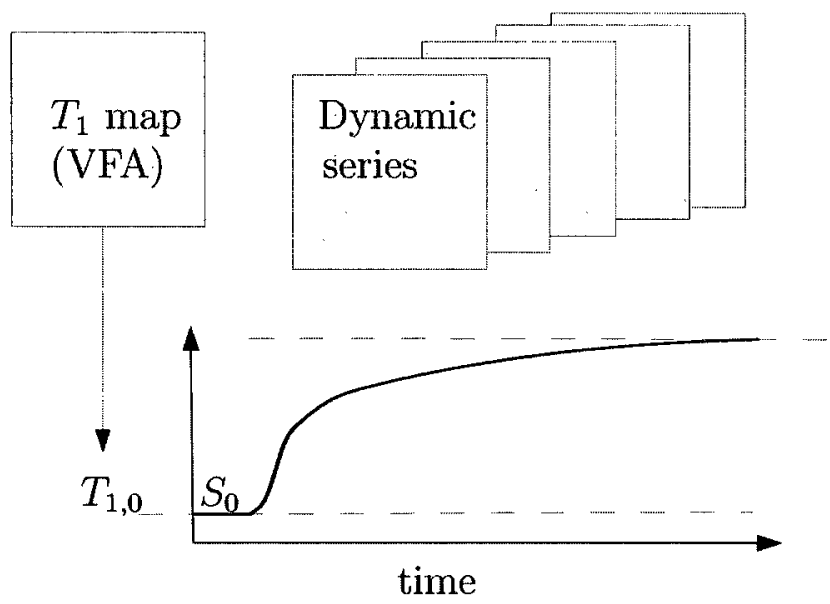

Figure 2.16: Schematic of a DCE-MRI acquisition with a pre-contrast $T_{1}$ map acquired using a variable flip angle (VFA) approach.

\section{$T_{1}$ pre method}

This method of signal to [CA] conversion assumes that the flip angle is known throughout the whole volume; either it is uniform or a pixel-by-pixel flip angle map is known. Additionally, it assumes that $T_{1}$ changes with [CA] but that $T_{2}^{*}$ remains constant (i.e. $M_{0}^{\prime}$ remains constant). This assumption is reasonable if the concentration is not too high and the $T_{E}$ is small. This is approximately true in most of the tissue since the $[\mathrm{CA}]$ generally remains below $2 \mathrm{mM}$; it is, however, not true in the major blood vessels. To remove the effect of the $T_{2}^{*}$ decay, a double echo sequence can be applied and the data extrapolated to $T_{E}=0 \mathrm{~ms}$.

Let the signal in the presence of the CA at a concentration of $C$ be $S_{C}$, and let the signal in the absence of the CA be $S_{0}$. From Eq. 2.83 the relative difference in the signal is

$$
\frac{S_{C}-S_{0}}{S_{0}}=\frac{\left[\frac{\left(1-E_{1, C}\right)}{\left(1-\cos \alpha E_{1, C}\right)}-\frac{\left(1-E_{1,0}\right)}{\left(1-\cos \alpha E_{1,0}\right)}\right]}{\frac{\left(1-E_{1,0}\right)}{\left(1-\cos \alpha E_{1,0}\right)}}
$$


where, based on Eqs. 2.78 and $2.107, E_{1, C}$ is defined as

$$
E_{1, C}=\exp \left[-T_{R}\left(C \lambda_{1}+\frac{1}{T_{1,0}}\right)\right]
$$

Let

$$
A_{0}=\frac{\left(1-E_{1,0}\right)}{\left(1-\cos \alpha E_{1,0}\right)}
$$

and let

$$
A=A_{0} \frac{S_{C}}{S_{0}} .
$$

Then Eq. 2.121 can be written as

$$
A=\frac{\left(1-E_{1, c}\right)}{\left(1-\cos \alpha E_{1, c}\right)}
$$

Solving for $C$ gives

$$
C=\frac{1}{\lambda_{1}}\left\{\frac{-1}{T R}\left[\ln \left(\frac{1-A}{1-A \cos \alpha}\right)\right]-\frac{1}{T_{1,0}}\right\}
$$

The signal strength for the SPGR sequence is approximately linear with [CA] at low concentrations, as can be seen in Fig. 2.11.

\section{Bookend Method}

The signal to [CA] conversion method presented above assumes that $T_{R}, T_{E}$, and $\alpha$ are all well known. While this will always be true for the timing parameters, the flip angle can have a large uncertainty associated with it [33]. The flip angle will, in general, vary both in-plane and across the slice (see Section 2.4.2). The flip angle for the $i^{\text {th }}$ voxel, $\alpha_{i}$, will not be exactly equal to the nominal flip angle, $\alpha$ entered into the user interface on the MRI scanner. The distribution of flip angles across the slice means that spins in different locations within the same voxel will not necessarily 


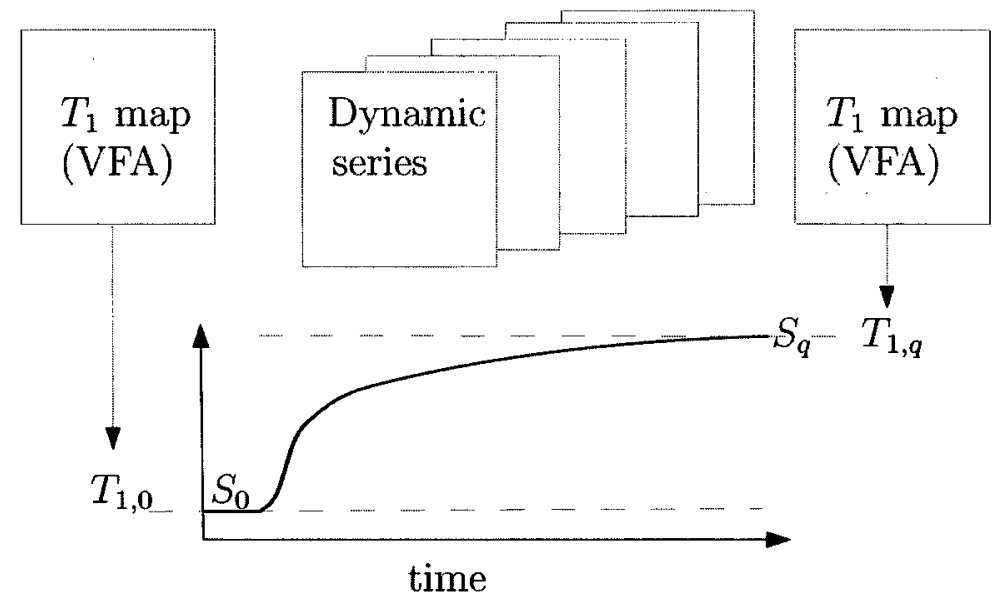

Figure 2.17: Schematic of a DCE-MRI acquisition with bookend $T_{1}$ maps acquired using a variable flip angle (VFA) approach.

experience the same degree of excitation. Thus, the signal measured from the $i^{\text {th }}$ voxel is the sum of the signals generated for a distribution of flip angles across the slice. Since the SPGR signal equation is not a linear function of $\alpha$, the signal from the $i^{\text {th }}$ voxel will not be equivalent to the signal that would be obtained if all of the spins in the voxel experienced the same flip angle corresponding to $\left\langle\alpha_{i}(z)\right\rangle_{z}$, the mean flip angle for the voxel:

$$
S_{i}=\int S_{i}\left(\alpha_{i}(z)\right) \mathrm{d} z \neq \int S_{i}\left(\left\langle\alpha_{i}(z)\right\rangle_{z}\right) \mathrm{d} z
$$

In the case of 3D acquisitions, if the slices on the outside of the slice block are ignored, the flip angle profile in the rest of the volume is relatively uniform. In the case of $2 \mathrm{D}$ acquisitions the non-uniformity is significant.

One approach that does not require knowledge of the exact slice profile (i.e. distribution of flip angles across the slice) or a $B_{1}$ inhomogeneity map is to apply what is called the Bookend method developed by Cron et al. [34]. This method involves the acquisition of an additional $T_{1}$ map, measured after the contrast bolus has passed through the tissue and the enhancement has reached a quasi-equilibrium state (see 
Fig. 2.17). The measurement technique is otherwise the same as that presented above and again, a double echo is preferable if $T_{2}^{*}$ is expected to affect the signal significantly. The following derivation explains how the additional $T_{1}$ measurement is incorporated into the calculation to improve the signal-to-concentration conversion.

Consider Eq. 2.117 for a case where the [CA] is known, but the true flip angle distribution does not match the nominal distribution as described above. Since the flip angle is not well known, this is essentially an estimate of the true signal:

$$
S_{\text {estimated }}=M_{0} f(C)
$$

This is different from the true signal equation by some function scaling factor, $P^{\prime}$, where $P^{\prime}$ could be a function of flip angle for example. Thus, the true signal can be written as

$$
S_{\text {true }}=P^{\prime} S_{\text {estimated }}
$$

Then the two scale factors can be combined so that

$$
S_{\text {true }}=P^{\prime} M_{0} f(C)=\operatorname{Pf}(C)
$$

where $P=P^{\prime} M_{0}$. Through simulation, Cron et al. [34], found that $P$ varies approximately linearly with $T_{1}$ for a 2D-SPGR sequence. The bookend $T_{1}$ maps, combined with the dynamic 2D-SPGR signal in the absence of CA, $S_{0}$, and at the quasistatic equilibrium, $S_{q}$, can be used to calculate an equation for $P\left(T_{1}\right)$. First calculate $P$ at the two bookends:

$$
\begin{aligned}
S_{0}, T_{1,0} \rightarrow P_{0} & =\frac{S_{0}}{f\left(C_{0}\right)} \\
S_{q}, T_{1, q} \rightarrow P_{q} & =\frac{S_{q}}{f\left(C_{q}\right)} .
\end{aligned}
$$


Then, apply the equation of a line through two points to get the relation for $P$

$$
P=\frac{P_{q}-P_{0}}{T_{1, q}-T_{1,0}} T_{1}-\frac{P_{q} T_{1,0}-P_{0} T_{1, q}}{T_{1, q}-T_{1,0}}
$$

This gives a function, $P\left(T_{1}\right)$, which can be converted into a function with [CA] as the dependent variable using Eq. 2.107. Thus, for a given voxel and a given time point in the set of $2 \mathrm{D}$ dynamic measurements, the change in the signal intensity from the pre-contrast baseline value can be related to the [CA] by curves similar to those shown in Fig. 2.11, but compensated for the inaccuracies of flip angle across the slice. Unfortunately, it is very difficult to solve Eq. 2.130 analytically for $C$. Thus, either iterative methods or a lookup table must be used to convert the measured signal intensities to $[\mathrm{CA}]$. In practice, iterative techniques tend to be too slow, and the lookup table approach is the preferred method.

This can be applied to the 2D dynamic series. The dynamic series must be long enough that the concentration has reached quasi-equilibrium by the end of the experiment so that the signal at equilibrium can be estimated and that the [CA] is constant while the post-contrast $T_{1}$ data is acquired. 


\section{Chapter 3}

\section{Motivation and Proposed Research}

DCE-MRI has the potential to provide valuable clinical information about the perfusion properties of tissues. This information is particularly useful in oncology because inadequate nutrient delivery limits a tumour's ability to grow. Although a simple visual analysis of the enhancement of the magnitude signal due to the presence of a contrast agent $(\mathrm{CA})$ can provide some information about the general characteristics of the tumour, tracer kinetic modelling is needed to relate the observed enhancement to the underlying physiology and to compare perfusion exam results from different patients. In order for the perfusion parameters measured from the tracer kinetic modelling to be accurate and reproducible, absolute quantification of the concentration of CA ([CA]) in the tissue as a function of time (TRF) and in a feeding artery as a function of time (AIF) are needed. The measurement of the AIF is the more challenging of the two since $[\mathrm{CA}]$ is higher in the AIF than it is in the TRF.

There are several ways of attempting to measure the AIF. Blood sampling is perhaps the most direct method for quantification of $[\mathrm{CA}]$, but it suffers from poor time resolution, there is the potential to disturb the hemodynamics of the subject [35] and it is invasive. Thus, blood sampling is not practical in many clinical situations. Another option is to generate a population averaged AIF. This can be done with either blood sampling [36, 37] or careful measurement using MRI, in a convenient vessel [38]. 
Advantages of this option are that, once the population AIF has been created, it is simple to use and, since the population AIF is less noisy than an individual AIF, it can improve reproducibility [38]. It can also be used even if there are no arteries in the imaging volume. The use of a population AIF assumes that the variation of the AIF within the population is low and that an AIF developed in one population can be applied to another. However, variations in cardiac output (inter- and intra-patient), kidney function, injection rate and body fat [35] can affect the AIF so there will be numerous situations when the use of the population AIF will lead to errors.

Finally, the AIF can be measured for each individual patient during the imaging exam. This is better than blood sampling because it is more convenient and noninvasive. It has the advantage over the use of a population AIF that it is measured directly for each patient. The disadvantages of this option are that there must be a suitable vessel available in the imaging volume and that the correct [CA] must be extracted. Conventionally, the changes induced in the magnitude of the MR signal, $|S|$, by the CA are used to measure the AIF. Unfortunately, this technique suffers from several weaknesses when quantitative values are desired. The phase of the MR signal, $\phi$, is also affected by the presence of the CA and has the potential to provide more accurate measurements of the AIF for several reasons discussed below.

The MR signal magnitude is nonlinear with [CA], in the case of gradient echo images, due to the non-linear relationship between $T_{1}, T_{2}^{*}$ and signal strength. As was shown in Section 2.8.3, because of the competing contrast between $T_{1}$ and $T_{2}^{*}$, the signal will saturate and begin to decrease, leading to a non univocal correspondence between signal and $[\mathrm{CA}]$ for concentrations greater than about $6.5 \mathrm{mM}$, for the range of sequence parameters used in this thesis. The non-linearity is most evident in the peak of the AIF, where a concentration of $7 \mathrm{mM}$ or above is expected, depending on the dose administered to the subject. One could try to avoid this by reducing the dose but this also reduces the contrast-to-noise ratio [39] for the TRF. Another option 
to reduce the effect of $T_{2}^{*}$ is to use a double echo sequence and extrapolate back to $T_{E}=0$, although this can reduce the time resolution and the non-linear relationship between $T_{1}$ and $|S|$ must still be considered.

The MR signal phase on the other hand, does not saturate at high [CA] and is linear with $[\mathrm{CA}][40]$. There can be some concern about phase wrapping leading to a non one-to-one correspondence between $\phi$ and $[\mathrm{CA}]$ but generally, the true concentration can be determined provided the change in phase between successive images, $\Delta \phi$, is less than $2 \pi$. Alternatively, the phase wrapping can be controlled by choosing a shorter echo time, $T_{E}$. The $T_{E}$ 's and time resolutions encountered in this thesis are sufficiently short that phase wrapping is not problematic and the true phase accumulation can be discerned.

The MR signal magnitude is sensitive to the flip angle used for excitation, (see Eq. 2.81). Because the radio frequency (RF) pulse is not infinite in length, there exist variations in the applied field both within and through the image plane (i.e. $\mathbf{B}_{\mathbf{1}}$ inhomogeneity) and thus the flip angle also varies. This leads to errors in the conversion from $|S|$ to $[\mathrm{CA}]$. For a more detailed discussion of this effect see Section 2.8.5. The $B_{1}$ inhomogeneity is reduced in the center of the imaging volume, where the tumour is typically located, but often a suitable vessel is not available in this location. Some authors generate a $B_{1}$ map to attempt to correct the image, but this generally requires additional scan time and complex calculations using the Bloch equations and knowledge of the exact slice profile. Alternatively, a bookend approach (see Section 2.8.5 and [41]), can be used to reduce the effect but this may not be sufficient at the edges of the image.

Conversely, the MR signal phase is insensitive to flip angle variations. Although the $\mathbf{B}_{\mathbf{1}}$ inhomogeneities lead to a variation in $\phi$ throughout the image, this $\phi$ offset is constant throughout the experiment. This is removed by acquiring baseline images pre-contrast injection and calculating $\Delta \phi$ between the image and the baseline. 
Baseline images are acquired for the standard DCE-MRI $|S|$ technique and thus this does not add any extra scan time. Subtraction of baseline images does not remove the effect of $\mathbf{B}_{\mathbf{1}}$ inhomogeneities on the conversion of $|S|$ to $[\mathrm{CA}]$ however, since it is dependent on the given flip angle in a nonlinear way, the effect cannot be subtracted off.

The MR signal magnitude is affected by blood flow into the volume of interest during the scan. The spins in the imaging volume are prepared by repeatedly applying an $\mathrm{RF}$ pulse, which tips the net magnetization vector towards the transverse plane, until it reaches a steady state (see Section 2.3.2). The signal strength equation of the spoiled gradient echo sequence is derived with the assumption that this steady state has been reached (see Section 2.6). However, during the acquisition of the image, spins in the blood flowing into the excited block, which have not been properly prepared by the train of RF pulses, contribute to the measured net magnetization leading to a signal that is not in a steady state. This leads to an apparent reduction in $T_{1}$, an effect that is greater for longer $T_{1}$. Thus, the $\Delta S$ measured in the dynamic series in a vessel does not correspond to the correct changes in the relaxation times resulting in a reduction in the enhancement and an underestimation of [CA]. This effect is more significant at the inflow edge of the imaging volume, for smaller imaging volumes and for $2 \mathrm{D}$ acquisitions. One strategy to reduce blood flow effects is to use a large imaging volume and to carefully place the region of interest (ROI) used to measure the AIF beyond the distance blood can travel by the time the maximum image contrast is acquired (i.e. when the center of $\mathrm{k}$-space is recorded) [35]. However, this limits the vessels available to be used for an AIF and large imaging volumes take longer to acquire, reducing the time resolution. A poor time resolution can negatively impact the tracer kinetic modelling.

The MR signal phase is relatively unaffected by moderate blood flow $(<15 \mathrm{~cm} / \mathrm{s})$ $[41,29]$. This is because the incoming magnetization does not have a component in 
the transverse plane and so does not contribute to the angle of the vector, only its magnitude. At very high flow rates, there can be sufficient loss of magnitude which will also cause a reduction of the phase SNR.

Finally, accurate conversion of $|S|$ to $[\mathrm{CA}]$ in blood vessels requires knowledge of the pre-contrast $T_{1}$ values in the blood. Because of the blood flow and $B_{1}$ inhomogeneity effects, the pre-contrast $T_{1}$ calculated using the variable flip angle (VFA) method (see Section 2.8.4) in blood vessels may not be accurate and thus the $T_{1}$ of blood must be estimated based on literature values. The amount of phase accumulation is not dependent on pre-contrast properties of the tissue and thus information from pre-contrast scans is not needed (aside from the baseline dynamic images).

In summary: 1) MR signal magnitude is nonlinear with [CA] and saturates at high [CA], 2) MR signal magnitude is sensitive to $B_{1}$ inhomogenieties (i.e. errors in flip angle), 3) MR signal magnitude is affected by blood flow into the volume of interest during the scan and 4) MR signal magnitude is dependent on the pre-contrast $T_{1}$. In contrast: 1) MR signal phase is linear with [CA] provided the $T_{E}$ has been chosen to be small enough that $\phi$ wrap is not a problem, 2) MR signal phase in insensitive to $B_{1}$ inhomogeneities, 3) MR signal phase is insensitive to moderate blood flow and 4) MR signal phase does not depend on the pre-contrast properties of the tissue.

\subsection{Literature Review}

Despite the expected advantages of $\phi$ for the accurate measurement of the AIF, this technique has received little attention in the literature. Initial investigations into the use of $\phi$ to measure [CA] involved relatively long echo times $\left(T_{E}\right)$ and limited in vivo experiments. Despite these limitations they provided encouraging results for the $\phi$ AIF approach. Conturo et al. (1992) [42] studied phase changes induced by a Gdbased contrast agent in vials oriented perpendicular to the magnetic field for $T_{E}$ 's as 
short as $5 \mathrm{~ms}$ and in the basal ganglia of two baboons in vivo at $T_{E}=22 \mathrm{~ms}$, both at 1.5 T. Akbudak et al. (1996) [43] applied the same technique to one baboon aorta (6-7 $\mathrm{mm}$ in diameter) oriented parallel to the magnetic field with an in-plane resolution of $1.87 \times 2 \mathrm{~mm}^{2}$ for $T_{E}=48.1 \mathrm{~ms}$ at $1.5 \mathrm{~T}$. Conturo et al. (2005) also showed that this technique can be applied to a baboon carotid artery at $3 \mathrm{~T}$ with $T_{E}=13 \mathrm{~ms}$ and an in-plane resolution of $2.5 \times 2.5 \mathrm{~mm}^{2}$. The effect of flow on phase measurements was shown to be minimal by Akbudak et al. (1997) using a cylindrical flow phantom. As well, through simulation, Kotys et al. (2007) saw an approximately 10 fold increase in SNR when using $\phi$ as compared to $|S|$ in $T_{2}^{*}$-weighted imaging at $3 \mathrm{~T}$ and $T_{E}=14 \mathrm{~ms}$. In addition, van Osch et al. used $\phi$ to correct AIF partial volume artifacts in human carotid arteries for $T_{2}{ }^{*}$-based DSC-MRI $[44,45,46]$, but did not use $\phi$ directly.

More recently, at least three research groups have investigated $\phi$-derived AIFs at the shorter $T_{E}$ 's ( $\leq 3 \mathrm{~ms}$ ) used in DCE-MRI studies, although this is very uncommon. Cron et al. (2005) measured $\phi$-derived AIF's in the abdominal aortas of rats for $T_{E}=3$

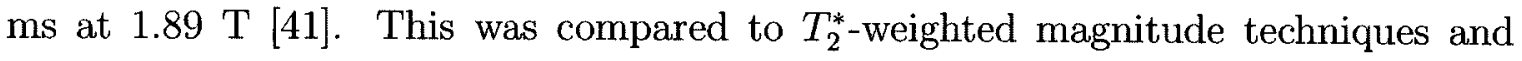
found to be more reliable when both were compared to blood samples. Ribot et al. (2008) used $\phi$ to measure AIF's in mice at $4.7 \mathrm{~T}$ for $T_{E}=1.66 \mathrm{~ms}$ (16 averages) and $T_{E}=3.32 \mathrm{~ms}$ using Dy-DOTA as the CA [47]. In this case, the vessel geometries were not considered. de Rochefort et al. (2008) measured $\phi$-derived AIFs in the human aorta with $T_{E}=3.3 \mathrm{~ms}$ at $1.5 \mathrm{~T}$ for 14 cases [29]. The vessel geometry was considered using a Maxwell boundary element method proposed by Salomir et al. [48].

This preliminary work is very encouraging and shows that the use of $\phi$ has the potential to be a useful solution to the well accepted and common problem of measuring an accurate AIF in DCE-MRI. Despite these encouraging results, $\phi$ imaging of the AIF has not been widely implemented. This may be in part because the current literature does not provide sufficient validation of the technique for its integration into common DCE-MRI protocols. The current literature outlined above involves a 
relatively small number of subjects, particularly human subjects and these studies, for the most part, deal with relatively ideal phase conditions; they have studied large vessels at good resolution and have not explored the limits of vessel diameter to resolution in terms of partial volumes and signal leakage. As well, most studies have used relatively long echo times. These echo times are not feasible for DCE-MRI studies, which require good time resolution and do not use longer $T_{E}$ EPI-based sequences (as is the case in $T_{2}^{*}$-weighted techniques) which are inherently extremely fast. Most studies do not fully address the implementation of measurement of the geometry factor, as they deal with cylinders, straight vessels or simply do not consider the effects of geometry.

\subsection{Outline of Proposed Research}

The main goal of this thesis is to demonstrate the feasibility of using $\phi$ in DCE-MRI of human subjects in clinically relevant situations and to demonstrate that this technique can be easily included in a standard clinical DCE-MRI exam without compromising the information currently derived from the technique or adding significant length to the duration of the scan. To do this, the technique must be tested over an extended range of vessel size, resolution and echo time, a user-friendly method of including the geometry factor must be developed and the application of the technique to clinical human studies of DCE-MRI, including the assessment of the perfusion parameters, must be explored.

To extend the use of the $\phi$ technique for use clinically, it must first be tested in phantoms. The effects of partial volumes and signal leakage on $\phi$ measurements have not been reported in the literature for a range of vessel sizes even though in clinical applications large vessels are not always available. These effects were studied by varying the field of view (FOV) and the wall thickness of a blood vessel phantom 
as part of the research reported in this thesis. The effect of changing the FOV is comparable to reducing the size of the vessel if the matrix size is held constant.

The effect of the partial volumes and signal leakage may be different at different levels of $\mathrm{SNR}_{\phi}$ which is dependent on both $T_{E}$ and $B_{0}$. A shorter $T_{E}$ means there is less time for $\phi$ accumulation and a lower $B_{0}$ means that the frequency at which the $\phi$ accumulates is reduced. Thus, the combination of $T_{E}$ and $B_{0}$ used clinically should be tested. To study these effects, a cylindrical blood vessel phantom with a range of $[\mathrm{CA}]$ was imaged to assess the conversion of $\phi$ to $[\mathrm{CA}]$ for several combinations of FOVs and $T_{E}$ 's. The study used a straight cylindrical phantom oriented at different angles with respect to the main magnetic field, $\mathbf{B}_{0}$, as well as a curved cylindrical phantom. The phantoms were always imaged perpendicular to the magnetic field and not perpendicular to the vessel so that any partial volume effects in the slice direction are included. A thin walled $(0.3 \mathrm{~mm})$ phantom was also investigated as a thicker walled phantom can reduce the effect of partial volumes and signal leakage since the wall material does not contribute to the signal. The effect of blood flow on $\phi$ has already been investigated and will not be addressed here $[49,41,29]$. The results of these phantom studies are presented in Chapter 4.

Most studies have confined their work to straight cylindrical shapes, usually without considering the orientation of the cylinder with respect to the magnetic field. Recently, deRochefort et al. (2008) [29] did include a calculation of the geometry factor in their measurement, but the implementation is complex. Another method in the literature, provided by Marques and Bowtell (2005) [25] presents a simpler technique for estimating magnetic susceptibility maps (see Section 2.7.3). This method may be applicable to the case of measuring contrast enhancement [50], although it has not been validated directly in the literature. One goal of this thesis is to effectively deal with the geometry factor in a way that is accurate but requires minimal user interaction, to make it practical for clinical use. In Chapter 5, the method proposed 
by Marques and Bowtell is validated for the case of contrast enhancement using simulations and phantom experiments. In addition, the feasibility of this technique for clinical data is demonstrated. The clinical application of this method was performed using images which are currently part of the standard imaging protocol and thus, does not require additional scan time.

As an alternative to modelling the geometry factor explicitly, approximation methods can be applied for simple vessel geometries. In this thesis, an approximation method referred to as "the straight tube approximation" is proposed. This method involves measuring the angle between the tangent to the vessel and the magnetic field and then calculating the geometry factor by assuming a cylindrical vessel of infinite extent at that angle. This tangential angle can be measured directly from an image or by applying a polynomial fit to points drawn along the vessel and extracting the tangent from the first order derivative. Both methods were explored using simulations of different curved geometries (Chapter 5), as well as through phantom experiments with curved geometries (Chapter 4).

An important part of this thesis is applying the $\phi$ method to human studies, using protocols which are clinically relevant to DCE-MRI. The use of $\phi$, at short $T_{E}$ 's, to measure the AIF has received very limited attention, and for only a small number of patients and large vessels. Comparison of the resulting perfusion parameters and of perfusion parameters derived using $|S|$ have not been reported previously. Thus, the use of $\phi$ in DCE-MRI for two clinical trials, a brain tumour study and a prostate tumour study, are presented. The first study is an ongoing 2D DCE-MRI study of brain tumour patients and the preliminary results presented here are for a relatively small number of patients. The exam consists of several slices through the tumour and superior sagittal sinus (SSS) and one slice through the neck. This additional neck slice allows a $\phi$-derived $\mathrm{AIF}, \mathrm{AIF}_{\phi}$, to be measured and compared to the venous output function (VOF) measured in the SSS. To our knowledge, there are no published 
results of the simultaneous measurement of the carotid artery and the SSS using $\phi$ to compare the input curve to the output curve. These $\phi$-derived AIF's and time shifted VOF's were both used to generate perfusion parameters which were compared to determine if a time shifted VOF can be used in place of the AIF. This is useful if a suitable AIF is not available in the imaging volume, but concerns that differences in shape between the VOF and AIF will affect the perfusion results must be addressed. A $|S|$-derived $\mathrm{AIF}, \mathrm{AIF}_{|S|}$, cannot be measured in a single slice, due to blood flow effects. Thus the $\mathrm{VOF}_{\phi}$ and $\mathrm{VOF}_{|S|}$, along with the perfusion parameters computed with these functions, were compared. In addition, the measured AIF's and VOF's were compared to a population AIF from the literature [38].

To our knowledge, the use of $\phi$ to measure the AIF has not been investigated in DCE-MRI studies of the pelvis. This imaging volume generally contains the iliac and/or femoral artery, making it an attractive candidate for this application. There are, however, several additional challenges that must be addressed for DCE-MRI studies of extra-cranial tumours. In particular, there can be more motion; Padhani et al. reported significant motion due to peristalsis of the rectum [51]. As well, the SNR from the body coil is less than that of the head coil, making both the $|S|$ and $\phi$ measurements more challenging. As one of the goals of this work is to challenge the $\phi$ technique, this study employed a $3 \mathrm{D}$ acquisition and a shorter $T_{E}$ than was used in the brain study. A 3D sequence will have regions of the imaging volume where the effects of blood flow and $B_{1}$ inhomogeneities, which corrupt the $|S|$, will be reduced as compared to a $2 \mathrm{D}$ study. Nonetheless, it is expected that the $\mathrm{AIF}_{|S|}$ will vary with the location of the measurement, whereas the $\mathrm{AIF}_{\phi}$ will not. The effect of this on the resulting perfusion parameters was assessed and the results are presented in Chapter 6.

In summary, this work aims to show that $\mathrm{AIF}_{\phi}$ is far superior to $\mathrm{AIF}_{|S|}$ for human studies. It is expected that the $\mathrm{AIF}_{\phi}$ will compare favourably with the population 
AIF reported by Parker et al. but, since it is measured directly for each subject, there will be no concern over whether the AIF is appropriate for a given subject. The introduction of $\mathrm{AIF}_{\phi}$ into DCE-MRI measurements would be a very significant and promising advance in the field of perfusion MR imaging. 


\section{Chapter 4}

\section{Phantom Experiments}

\subsection{Introduction}

The quantitative measurement of tissue perfusion requires an accurate measure of the concentration of contrast agent (CA) in the tissue. It also requires an accurate knowledge of the concentration of $\mathrm{CA},[\mathrm{CA}]$, in a feeding artery (AIF). Both the magnitude, $|S|$, and the phase, $\phi$, of the MRI signal are affected by the presence of CA and, in principle, either can be used to estimate the $[\mathrm{CA}]$ contained in a vessel. As discussed in Chapter 3, there are several advantages to using $\phi$ over $|S|$ when measuring [CA] in large vessels; the conversion is linear, it does not saturate at high concentrations, it is insensitive to flip angle inhomogeneities and it is relatively insensitive to blood flow.

The primary objective of this thesis is to demonstrate that $\phi$ can be used to calculate $[\mathrm{CA}]$ in a vessel and that it is, in fact, preferable to use $\phi$ rather than $|S|$. The first step in testing this hypothesis is to validate the $\phi$-to- $[\mathrm{CA}]$ conversion in a controlled experiment. This is the subject of this chapter. Validation is achieved by studying a blood vessel phantom with known [CA] in a variety of situations that mimic those that arise clinically. The situations tested included: 1) different vessel 
orientations and curvatures to assess the geometry factor (see Section 2.8.2), 2) different spatial resolutions to assess the affect of partial volumes and signal leakage via the point spread function (see Section 2.8.3) and 3) different echo times to assess the effect of the phase signal-to-noise ratio $\left(\mathrm{SNR}_{\phi}\right)$ (see Section 2.5.2 for a discussion of $\mathrm{SNR}_{\phi}$ ). The variable flip angle method for measuring $T_{1}$ (see Section 2.8.4) was also validated by comparing the $T_{1}$ values measured with this technique, as a function of $[\mathrm{CA}]$, with the results obtained using an inversion recovery sequence. Relaxivity values were also calculated for both techniques and compared with values from the literature.

\subsection{Method}

\subsubsection{Blood Vessel Phantom}

A blood vessel phantom was constructed using a sealed length of flexible polyethylene tubing. The tubing had an inner diameter of $9 \mathrm{~mm}$, an outer diameter of $13 \mathrm{~mm}$, and was $180 \mathrm{~mm}$ long. The phantom was filled with known concentrations of the contrast agent, Gadovist@ (Bayer Healthcare). A [CA] range from 0-10 mM was used (see Table 4.1 for further details). This is the approximate range of concentrations expected in the blood vessels during the course of a typical DCE-MRI study [38].

The blood vessel phantom was placed in a water bath. The bath was doped with CA to lower its $T_{1}$ to approximately $700 \mathrm{~ms}$, to simulate tissue. In this experiment, water was used instead of blood because of the ease of manipulation and availability. The magnetic susceptibility changes are approximately the same in water as they are in blood $[41,46,40]$, and thus the results are transferable to the case of CA doped blood. To test the effect of geometry, a curved tube phantom with a radius of curvature of $45 \mathrm{~mm}$ was also studied. This curvature was chosen because it is 
expected that the superior sagittal sinus, an important component of the brain study (Chapter 6), will in general have a radius of curvature between infinity (straight) and $45 \mathrm{~mm}$. The straight tube phantom was imaged at multiple angles ranging from $0^{\circ}$ to $20^{\circ}$ (see Table 4.1 for more details).

An additional plastic thin walled tube was used to study the effect of the wall thickness on the phantom measurements. This phantom was also surrounded by doped water. The inner diameter was $10.3 \mathrm{~mm}$ and wall thickness was $0.3 \mathrm{~mm}$. Since the phantom material was not flexible, only straight tubes were used.

\subsubsection{Phase Data Acquisition}

The MRI protocol used in this blood vessel phantom experiment was set up to mimic the protocol that would be used in the brain study described in Chapter 6. All imaging was performed on a 1.5 T Siemens Symphony using the standard CP Head Array coil (Siemens Medical Solutions, Erlangen, Germany) with software version A25. A dual-echo 2D spoiled gradient echo (SPGR) pulse sequence was used with $T_{R}=45 \mathrm{~ms}, T_{E}=(2.06 \mathrm{~ms}, 5.48 \mathrm{~ms})$ and a flip angle of $90^{\circ}$. The image matrix size was $96 \times 128$ (48 × 128 before online zero-filling). The field of view (FOV) was asymmetric, with the size in the phase encoding direction being 0.75 times the size in the frequency encoding direction in all cases; see Table 4.1, for specific values. The spatial resolution quoted in Table 4.1, is defined using the original $48 \times 128$ images with no interpolation ( $\mathrm{k}$-space zero-filling). Nine images, averaged offline, were used to assess the variation in the phase data and to improve the $\mathrm{SNR}_{\phi}$ of the lower [CA] data points. Raw data were stored during the image acquisition.

This protocol was applied to each [CA]-geometry combination described in Table 4.1. The slice thickness for the straight tube experiments was $10 \mathrm{~mm}$, taken at the midpoint of the tube. The slice thickness used in the curved tube experiments was reduced to $5.5 \mathrm{~mm}$ and 9 slices were acquired $2.75 \mathrm{~mm}$ apart. The midpoint of the 


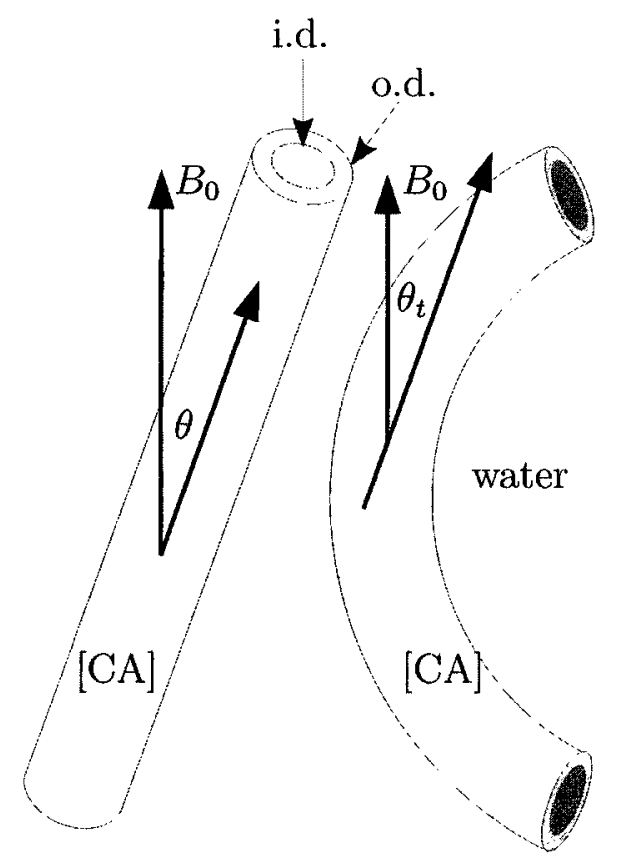

Figure 4.1: Schematic showing phantom experiments for straight and curved tubes. The i.d. is inner diameter and o.d. is outer diameter.

curved tube was positioned to be parallel to the external magnetic field, with the curve in the coronal plane. All images were in the transverse orientation. For the curved blood vessel phantom, the tangential angle between the vessel and the main magnetic ficld was determined using a coronal image.

The thin walled tube phantom was imaged with the same protocol for a subset of the experiments, to explore the potential effect of the wall thickness on partial volume and signal leakage effects. This phantom was imaged for all concentrations, FOVs and $T_{E}$ 's but, for $0^{\circ}$ and $15^{\circ}$ only.

\subsubsection{Phase Analysis Method}

All processing of the MRI signal phase was performed offline using custom software

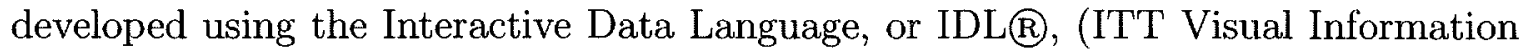
Solutions). Data collected with an FOV $<300 \times 400 \mathrm{~mm}^{2}$ was analyzed using the 
Table 4.1: Summary of experimental parameters. The spatial resolution (SR) was calculated from the non-zerofilled matrix size.

\begin{tabular}{|c|}
\hline$[\mathrm{CA}](\mathrm{mM})$ \\
0.05 \\
0.125 \\
1 \\
2 \\
3 \\
4 \\
6 \\
8 \\
10 \\
\hline FOV $\left(\mathrm{mm}^{2}\right)$ SR $\left(\mathrm{mm}^{2}\right)$ \\
\hline $150 \times 2.13 \times 1.56)$ \\
$225 \times 300 \quad(4.69 \times 2.34)$ \\
$300 \times 400 \quad(6.25 \times 3.13)$ \\
375 x $500 \quad(7.81 \times 3.91)$ \\
\hline$T_{E}(\mathrm{~ms})$ \\
\hline 2.06 \\
5.48 \\
\hline Geometry \\
\hline straight $0^{\circ}$ w.r.t $\mathbf{B}_{\mathbf{0}}$ \\
straight $5^{\circ}$ w.r.t $\mathbf{B}_{\mathbf{0}}$ \\
straight $10^{\circ}$ w.r.t $\mathbf{B}_{\mathbf{0}}$ \\
straight $15^{\circ}$ w.r.t $\mathbf{B}_{\mathbf{0}}$ \\
straight $20^{\circ}$ w.r.t $\mathbf{B}_{\mathbf{0}}$ \\
curved \\
\hline
\end{tabular}


original $128 \times 48$ (non-zero-filled) raw data $\mathrm{k}$-space matrix. For FOV $\geq 300 \times 400 \mathrm{~mm}^{2}$, the voxel size was comparable to the cross-sectional area of the blood vessel phantom. In this case, error could result if the cross-sectional area inside the phantom straddled two or more pixels in the original non-zero-filled image. Thus, for these FOVs, each raw data set was zero-filled to provide an interpolated image matrix size of $384 \times 144$. Finally, in all cases a 2D inverse Fourier transform of the k-space data was performed to obtain the complex image data, from which the $|S|$ and $\phi$ were calculated.

Where necessary, phase unwrapping was performed. Phase unwrapping was performed by tracking the $\phi$ accumulation and ensuring that the $\Delta \phi$ between each $\Delta C$ was between $-\pi$ and $\pi$ by subtraction or addition of $2 \pi$ [44]. In cases where the phase accumulation has the potential to be $>2 \pi$ between consecutive $\Delta C$ measurements there can be ambiguity in the integer number of $2 \pi \mathrm{s}$ which should be added or subtracted. For the echo times and concentration changes used in this experiment there was no ambiguity in the $\Delta \phi$ value. On each $|S|$ image, a small region of interest (ROI) (area $\approx 14 \mathrm{~mm}^{2}$ ) was drawn at the center of the blood vessel phantom and this location was transfered to the corresponding $\phi$ image. This ROI contained from 4 to 12 pixels for the zero-filled cases depending on the resolution being considered and 3 pixels for the non-zero-filled cases.

In Section 2.8.2 it was shown that the phase change of the MR signal, $\Delta \phi$ for a given voxel, due to the presence of a Gd-based MR CA, is linearly proportional to [CA], the concentration of contrast agent in the voxel. This theoretical relationship between $\Delta \phi$ and [CA] is given in Eq. 2.114. This equation can also be expressed in a form similar to the relaxivity equations (Eqs. 2.107 and 2.108) as:

$$
\phi(t)=\lambda_{\phi}\left(T_{E}, \theta\right) C+\phi_{0}
$$

where $\phi_{0}$ is the phase in the absence of contrast agent and $C$ is the [CA]. The slope, 
$\lambda_{\phi}\left(T_{E}, \theta\right)$, is given by:

$$
\lambda_{\phi}\left(T_{E}, \theta\right) \equiv \frac{\gamma \chi_{m}}{3} \frac{B_{0} T_{E}}{g(\theta)}
$$

where $\gamma$ is the ${ }^{\mathbf{1}} H$ gyromagnetic ratio and $\chi_{m}$ is the mass magnetic susceptibility for Gd. For each experimental set-up, a linear regression between $\phi$ and the known [CA] was calculated in order to quantify the relationship between $\phi$ and [CA]. The linear regression was weighted by the standard deviation (SD) measured from each ROI.

\subsection{4 $\mathrm{T}_{1}$ Measurement}

$T_{1}$ values for the blood vessel phantoms were also calculated. $T_{1}$ maps were generated using two methods: inversion recovery (IR) and variable flip angle (VFA). These measurements were used to test the VFA $T_{1}$ map method as well as to confirm that the $[\mathrm{CA}] \mathrm{s}$ in the phantoms were accurate. All of the tubes, each one with a different $[\mathrm{CA}]$, were imaged together in air. For the $T_{1}$ maps, there was no need to consider the orientation of the tubes although they were placed approximately lengthwise in the magnet.

The IR protocol consisted of a 2D-echo planar imaging (EPI) IR sequence with $T_{R}=10 \mathrm{~ms}, T_{E}=28 \mathrm{~ms}$. For further details on EPI based sequences see [20, p 524$533]$ and for more information on IR sequences see [21, p 221-223]. Forty-one different inversion times $\left(T_{I}\right)$, between $40 \mathrm{~ms}$ and $5000 \mathrm{~ms}$, were applied in a random order. The FOV used was $230 \times 230 \mathrm{~mm}^{2}$ with a $10 \mathrm{~mm}$ slice thickness and a matrix size of 64 x 64 , leading to a resolution of $3.59 \times 3.59 \mathrm{~mm}^{2}$. An EPI-based sequence was chosen to keep the imaging time reasonable. EPI sequences can suffer from spatial distortions, but this was not a concern in this case since the tubes were easily identifiable, a single pixel ROI measurement was used and there was no need to register these images with the other images of the phantom.

Data was analyzed using Levenberg-Marquardt curve fit to the IR signal strength 
equation

$$
y=y_{0}+a * e^{-T_{I} / T_{1}}
$$

where $y_{0}, a$ and $T_{1}$ are the fitted parameters.

The VFA protocol consisted of a 3D SPGR sequence with $T_{R}=50 \mathrm{~ms}, T_{E}=2.16$ ms for 11 flip angles between $10^{\circ}$ and $70^{\circ}$. The FOV was $172.5 \times 230 \mathrm{~mm}^{2}$ with a 5 $\mathrm{mm}$ slice thickness, the matrix size was $96 \mathrm{x} 128$ with 20 slices leading to a resolution of $1.8 \times 1.8 \mathrm{~mm}^{2}$. This is the same protocol that was used for the brain study (see Chapter 6). The data was analyzed using the method described in Section 2.8.4.

\subsection{Results}

In this section, the results of the blood vessel phantom experiments are presented. As stated in the Methods section, a linear regression of $\Delta \phi$ versus [CA] was performed for each combination of geometry, $T_{E}$, and FOV given in Table 4.1. The slopes, $\lambda_{\phi}$, derived from these linear fits were used to assess the accuracy and precision of the $\phi$-to-[CA] conversion for these experimental conditions. All graphs in this section show the ratio of the experimental slope, $\left(\lambda_{\phi}\right)_{e x p}$, divided by the slope predicted by Eq. 2.113 for $\theta=0^{\circ}$ and given in Table 4.2. For all statistical tests presented in this work at sigificance level of $5 \%$ is assumed.

Table 4.2: Theoretical relationship between [CA], concentration of contrast agent ( $\mathrm{mM}$ ) and $\phi$ (radians) for the $T_{E}(\mathrm{~ms})$ used in the blood vessel phantom study.

\begin{tabular}{|cc|}
\hline$T_{E}(\mathrm{~ms})$ & $\left(\lambda_{\phi}\right)_{\text {theory }}(\mathrm{rad} / \mathrm{mM})$ \\
\hline 2.06 & 0.09369 \\
5.48 & 0.2492 \\
\hline
\end{tabular}




\subsubsection{Straight-Tube Phantoms}

The goal of this portion of the experiment was to validate the $\phi$-to- $[\mathrm{CA}]$ conversion method and to explore any variation in accuracy or precision with $\theta$, the orientation of the phantom with respect to the main magnetic field, the FOV or $T_{E}$. The expected result for a straight cylinder at an angle $\theta$ with respect to $\mathbf{B}_{0}$ is known analytically and thus the measured values can be compared to the theoretical result.

Two example graphs of $\phi$-to-[CA] are presented in Figs. 4.2 and 4.3 for the case when $\theta=0^{\circ}$ for $T_{E}=5.48 \mathrm{~ms}$ and $T_{E}=2.06 \mathrm{~ms}$, respectively. The results for $\theta>0^{\circ}$ are not shown but they are similar to the $\theta=0^{\circ}$ results. By comparing these graphs it can be seen that the variance is higher for the shorter $T_{E}$ case. This was the expected behaviour since $\mathrm{SNR}_{\phi}$ is smaller for $T_{E}=2.06 \mathrm{~ms}$ than it is for $T_{E}=5.48 \mathrm{~ms}$. For this reason the data for each $T_{E}$ were treated separately as the precision of the results was expected to differ. The slope, $\left(\lambda_{\phi}\right)_{\text {exp }}$, from each of these lines becomes one point in Figs. 4.4 and 4.5. These graphs show the expected increase in deviation from unity as $\theta$ increases.

These graphs show that a systematic error in the value of [CA] would occur if $\theta$ is ignored. It is often possible to measure the angle of the vessel from a $3 \mathrm{D}$ anatomic acquisition, which is becoming more commonly a part of the standard clinical routine. Thus, for the remainder of the analysis, it will be assumed that $\theta$ is known. The error in $\theta$ is not included in the following calculations but is estimated in this case to be approximately $\pm 1^{\circ}$ due to alignment and variation in the flexible tubing.

When $\theta$ is taken into account, as represented by the solid line in Figs. 4.4 and 4.5 , the results match very well with predictions. The average percent error between the measured and theoretical slope across all slopes (i.e. all $\theta$ and FOV values) for $T_{E}=5.48 \mathrm{~ms}$ was $(3 \pm 5) \%$ and for $T_{E}=2.06 \mathrm{~ms}$ was $(-3 \pm 11) \%$. Overall, the results are in very good agreement with theory. For some experiments the percent 


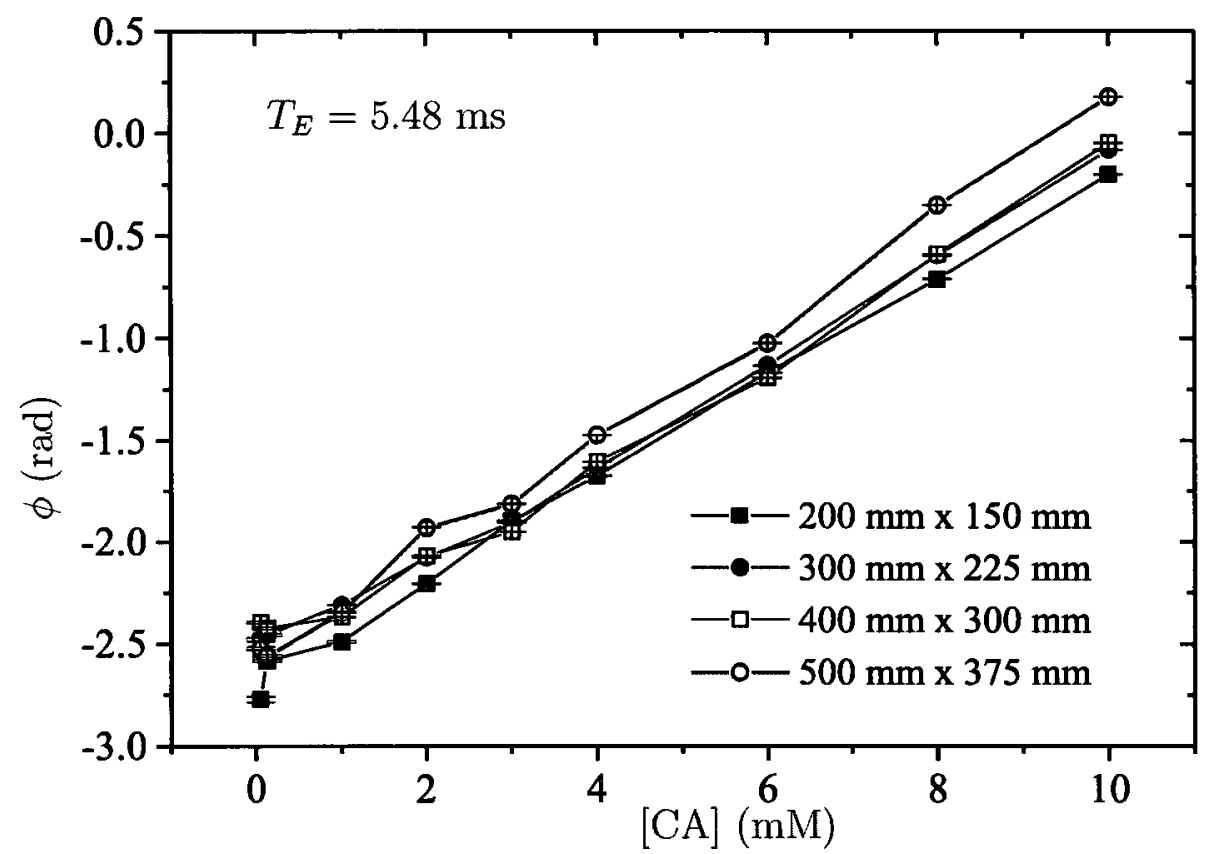

Figure 4.2: $\phi$ as a function of [CA] for a straight tube parallel to $B_{0} \hat{z}$ with $T_{E}=5.48$ ms for several FOVs. Error bars represent 1 SD of 9 image averages.

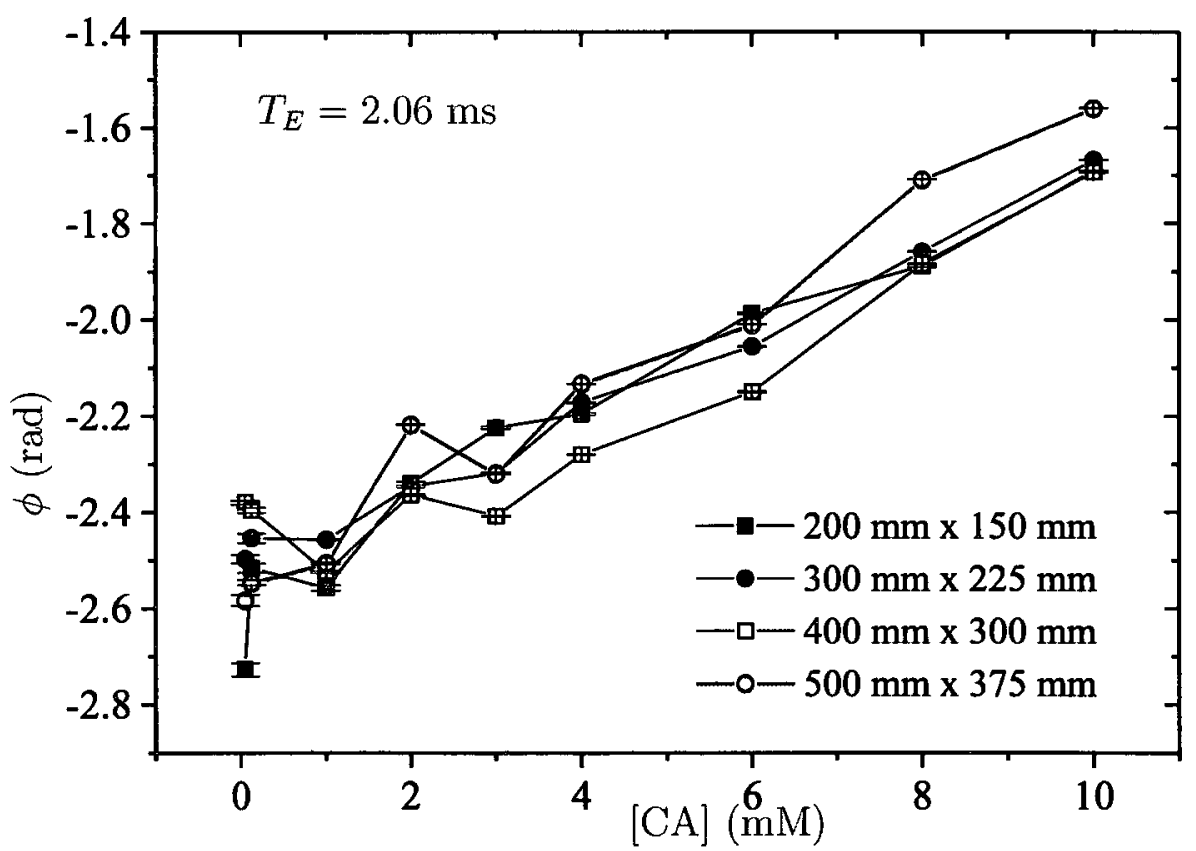

Figure 4.3: $\phi$ as a function of [CA] for a straight tube parallel to $B_{0} \hat{z}$ with $T_{E}=2.06$ ms for several FOVs. Error bars represent 1 SD of 9 image averages. 


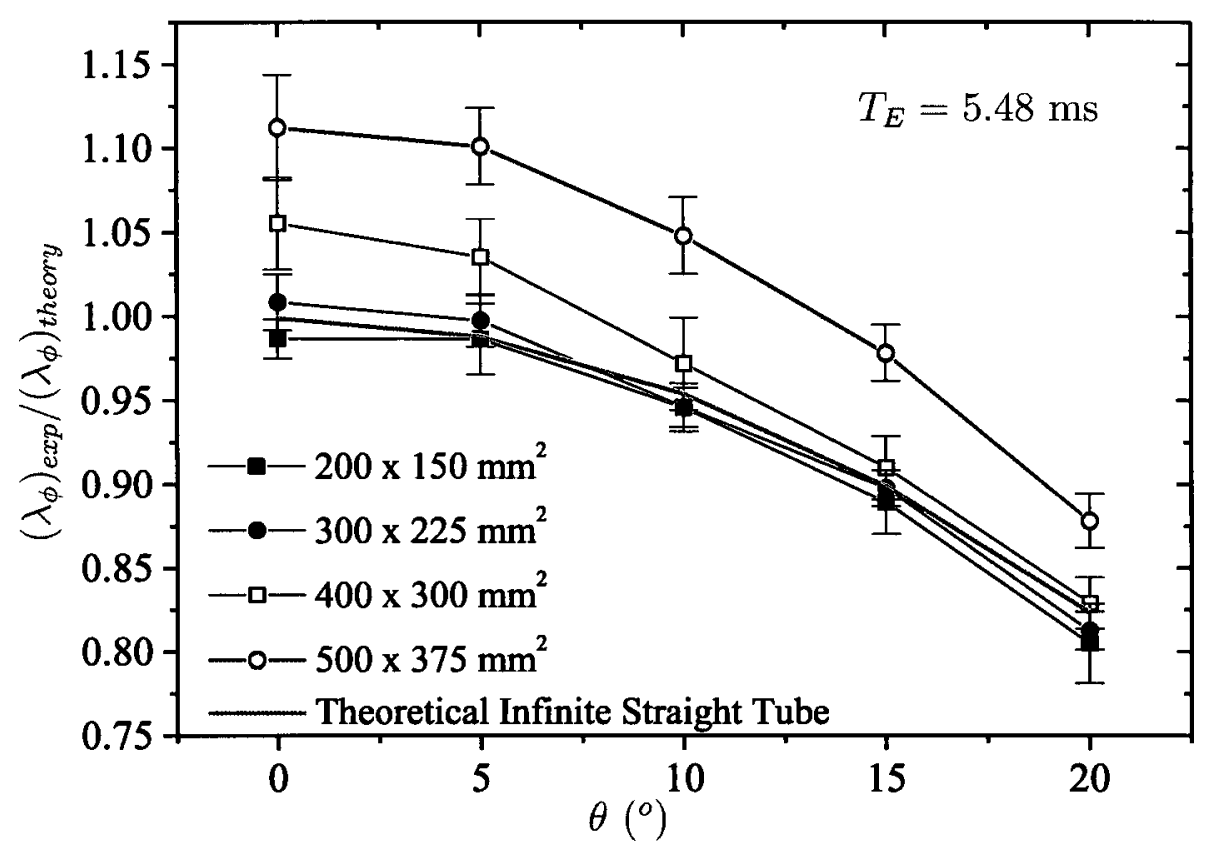

Figure 4.4: $\left(\lambda_{\phi}\right)_{\text {exp }} /\left(\lambda_{\phi}\right)_{\text {theory }}$ as a function of angle, $\theta$, for several FOVs for a straight tube parallel to $B_{0} \hat{z}$, with $T_{E}=5.48 \mathrm{~ms}$. The thick solid line shows the theoretical calculation from Eq. 2.113. The error bars represent the standard error calculated from the linear regression.

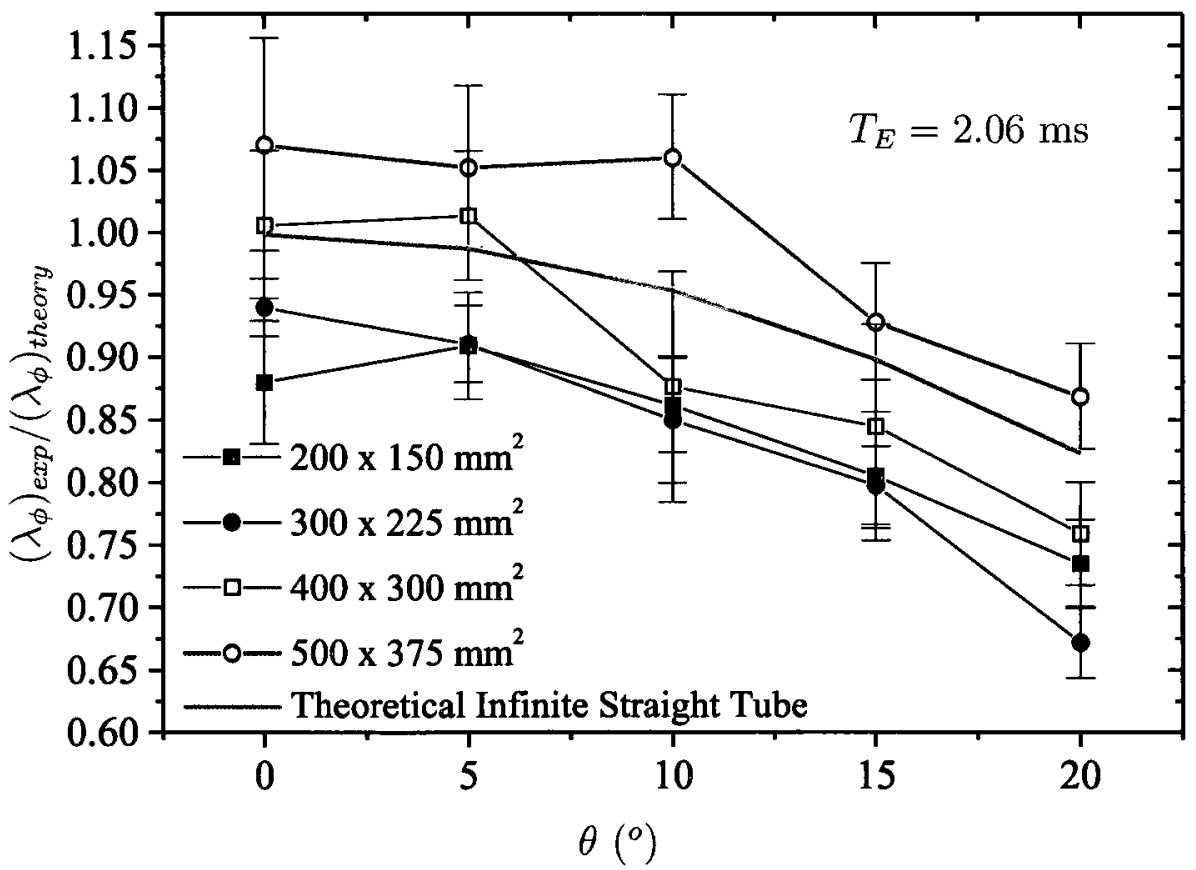

Figure 4.5: $\left(\lambda_{\phi}\right)_{\text {exp }} /\left(\lambda_{\phi}\right)_{\text {theory }}$ as a function of angle, $\theta$, for several FOVs for a straight tube parallel to $B_{0} \hat{z}$, with $T_{E}=2.06 \mathrm{~ms}$. The thick solid line shows the theoretical calculation from Eq. 2.113. The error bars represent the standard error calculated from the linear regression. 
differences are statistically significant (determined using a t-test), which means there is sufficient precision in the measurements to explore the variation of these percent differences with variations in experimental set-up. To assess the effect of various combinations of experimental set-up factors, two-factor analysis of variance (ANOVA) was performed on the slopes shown in Figs. 4.4 and 4.5. To facilitate the comparison of the slopes measured at different $\theta,\left(\lambda_{\phi}\right)_{\text {exp }}$ for each experiment was used to calculate the $[\mathrm{CA}]$ predicted by that slope for $1 \mathrm{mM}$ of $\mathrm{CA}$, incorporating the geometry factor. This is equivalent to Eq. 2.114 or

$$
C A_{1 m M}=\frac{1}{\left(\lambda_{\phi}\right)_{\exp }} \frac{\gamma T_{E} B_{0} \chi_{m}}{3} \frac{\left(3 \cos ^{2} \theta-1\right)}{2} * 1 m M
$$

The ANOVA between the slopes was calculated by comparing the mean square variance between linear fits to the mean square of the linear fit residuals using a Fdistribution and a null hypothesis of equal slopes. The results of this test are presented in Table 4.3 [52, p 292-301].

The results of the ANOVA show that the two factor interaction between FOV and $\phi$ is not significant for either $T_{E}$. The ANOVA also indicates that once $\theta$ is included, it does not influence the measured [CA]. The FOV size is a significant factor for both $T_{E}$ 's but the effect is stronger for the smaller $T_{E}$. A three-factor ANOVA also revealed that there is a statistically significant difference $(\mathrm{p}<0.0001)$ between the $\left[\mathrm{CA}_{1 m M}\right]$ measured using the two $T_{E}$ 's. Since $\theta$ does not produce a statistically significant difference in measured [CA], the $\left[\mathrm{CA}_{1 m M}\right]$ for a given $\theta$ were averaged and the final results are presented in Table 4.4 .

As stated above, the data derived from different $T_{E}$ 's have different variances. The COV for $\left[\mathrm{CA}_{1 m M}\right]$ averaged over all $\theta$ 's and FOVs for $T_{E}=2.06 \mathrm{~ms}$ had a mean and SD of $(5 \pm 2) \%$, whereas the value was $(2.0 \pm 0.5) \%$ for $T_{E}=5.48 \mathrm{~ms}$. It can be seen that the COV for the shorter $T_{E}$ is $2.8 \pm 1.3$ times larger than for the longer 
Table 4.3: Summary of 2 factor ANOVA results.

\begin{tabular}{|cccc|}
\hline Factor & $F$ & $\mathrm{p}$ & Conclusion \\
\hline \multicolumn{4}{c}{$T_{E}=2.06 \mathrm{~ms}$} \\
\hline and FOV interaction & 0.352 & 0.977 & Not significant \\
$\theta$ alone & 1.01 & 0.405 & Not significant \\
FOV alone & 18.2 & $<0.0001$ & Significant \\
\hline & $T_{E}=5.48 \mathrm{~ms}$ & \\
\hline and FOV interaction & 0.0193 & 1.00 & Not significant \\
$\theta$ alone & 0.191 & 0.944 & Not significant \\
FOV alone & 2.74 & 0.0455 & Significant \\
\hline
\end{tabular}

Table 4.4: Summary of [CA] (mM) predicted by phantom straight tube experiments, $\left[\mathrm{CA}_{1 m M}\right]$, at various $T_{E}$ 's and FOVs if $1 \mathrm{mM}$ of CA is present.

\begin{tabular}{|ccccc|}
\hline Echo Time $(\mathrm{ms})$ & FOV $\left(\mathrm{mm}^{2}\right)$ & FOV $\left(\mathrm{mm}^{2}\right)$ & FOV $\left(\mathrm{mm}^{2}\right)$ & FOV $\left(\mathrm{mm}^{2}\right)$ \\
& $200 \times 150$ & $300 \times 225$ & $400 \times 300$ & $500 \times 375$ \\
\hline 5.48 & $1.01 \pm 0.02$ & $1.00 \pm 0.014$ & $0.97 \pm 0.02$ & $0.91 \pm 0.02$ \\
2.06 & $1.11 \pm 0.06$ & $1.13 \pm 0.05$ & $1.04 \pm 0.08$ & $0.88 \pm 0.05$ \\
\hline
\end{tabular}


$T_{E}$. This is the expected result since the predicted phase SNR for the shorter $T_{E}$ is 2.7 times lower than that for the larger $T_{E}$.

\subsubsection{Curved-Tube Phantoms}

Generally, blood vessels are not perfectly straight. To explore the effect of curvature on the measurement of [CA] using $\phi$, the above analysis was reproduced for a curved tube with a $45 \mathrm{~mm}$ radius of curvature. In this work, the angle of the tube with respect to $\mathbf{B}_{\mathbf{0}}$, for any part of the tube, was taken as, $\theta_{t}$, the tangent to the axis of the tube, at that location: thus, it was possible to obtain the full angle dependency from a single experiment. The measured $\phi$ versus [CA] curves were similar to those shown in Figs. 4.2 and 4.3 for all $\theta_{t}$. As before, the observed variance for the short $T_{E}$ data was larger than for the large $T_{E}$, reflecting the larger $\mathrm{SNR}_{\phi}$ at larger $T_{E}$.

Unfortunately, a theoretical value for $\lambda_{\phi}$ for the curved vessel situation is unavailable so, to facilitate the analysis, it was hypothesized that the behaviour of the curved tube at a position with the tangential angle, $\theta_{t}$, would be equivalent to an infinitely long straight tube oriented at angle $\theta=\theta_{t}$ with respect to $\mathbf{B}_{0}$. Thus, the $\left(\lambda_{\phi}\right)_{\exp }$ shown in Figs. 4.6 and 4.7 are normalized to the infinite straight tube theoretical solution.

As was the case with the results from the straight tube experiment, the main factor influencing the deviation of the data points in Figs. 4.6 and 4.7 from unity is the tube angle. Again it is assumed that $\theta_{t}$ can be measured from a 3D anatomical acquisition. As before, the error on $\theta_{t}$ is not included in the following analysis. It is estimated to be $\pm 1^{\circ}$, found from calculating the average SD of the mean from three separate measurements of the tangent for all slices. The predicted result using the measured tangent is represented in Figs. 4.6 and 4.7 as a solid line.

It can be seen from the graphs, for all FOVs and $T_{E}$ 's studied, that the absolute difference between the measured and theoretical slopes does not change significantly 
for any of the angles measured. However, as $\theta_{t}$ increases, the expected slope decreases in magnitude with no increase in $\mathrm{SNR}_{\phi}$. As a result, for angles $30^{\circ}$ and above, there is a sharp increase in the COV. With this in mind, only data acquired for angles less than $25^{\circ}$ are used in the analysis given below. The percent error between measured values and theory, averaged over all FOVs and $|\theta| \leq 25^{\circ}$, for $T_{E}=5.48 \mathrm{~ms}$ was (-11 $\pm 5) \%$ and for $T_{E}=2.06 \mathrm{~ms}$ was $(-23 \pm 8) \%$. The measured value was lower than the theoretical value for all but one data point.

The data were rescaled to $\left[\mathrm{CA}_{1 m M}\right]$ as in the previous section describing the straight tube phantom. The two factor ANOVA revealed no statistically significant differences between experimental set-ups (all $p>0.88$ ), except for a small dependence on angle for $T_{E}=2.06 \mathrm{~ms}(p=0.0415)$. This suggests that perhaps there is some $\theta_{t}$ dependence at low $T_{E}$ 's; this is however a small effect. No statistically significant dependence on FOV was observed.

The $\left[\mathrm{CA}_{1 m M}\right] \mathrm{s}$ for each $T_{E}$ were pooled to form a weighted average. For $T_{E}=2.06$ $\mathrm{ms}$, the weighted mean and SD of the mean for $\left[\mathrm{CA}_{1 m M}\right]$ was $1.27 \pm 0.03 \mathrm{mM}$; a value of $1.10 \pm 0.01 \mathrm{mM}$ was found for $T_{E}=5.48 \mathrm{~ms}$. When compared to the pooled weighted average for straight tubes of $1.02 \pm 0.01 \mathrm{mM}$ for $T_{E}=2.06 \mathrm{~ms}$ and $0.982 \pm$ $0.004 \mathrm{mM}$ for $T_{E}=5.48 \mathrm{~ms}$, it appears there is some loss of accuracy in the curved measurements. Potential causes for this difference are discussed in Section 4.4.

The coefficients of variation $(\mathrm{COVs})$ of $\left[\mathrm{CA}_{1 m M}\right]$ were also calculated. For $T_{E}=$ $2.06 \mathrm{~ms}$ the COV has a mean and SD of $(11 \pm 3) \%$, and for $T_{E}=5.48 \mathrm{~ms}$ the COV was found to be $(5.4 \pm 1.4) \%$.

\subsubsection{Thin Walled Phantom}

This experiment was designed to study the effect of the wall thickness on the $\phi$ to $[\mathrm{CA}]$ conversion. The tubes analyzed in this experiment had a smaller wall thickness than the ones described above; the wall thickness for the thick walled tube was 2 


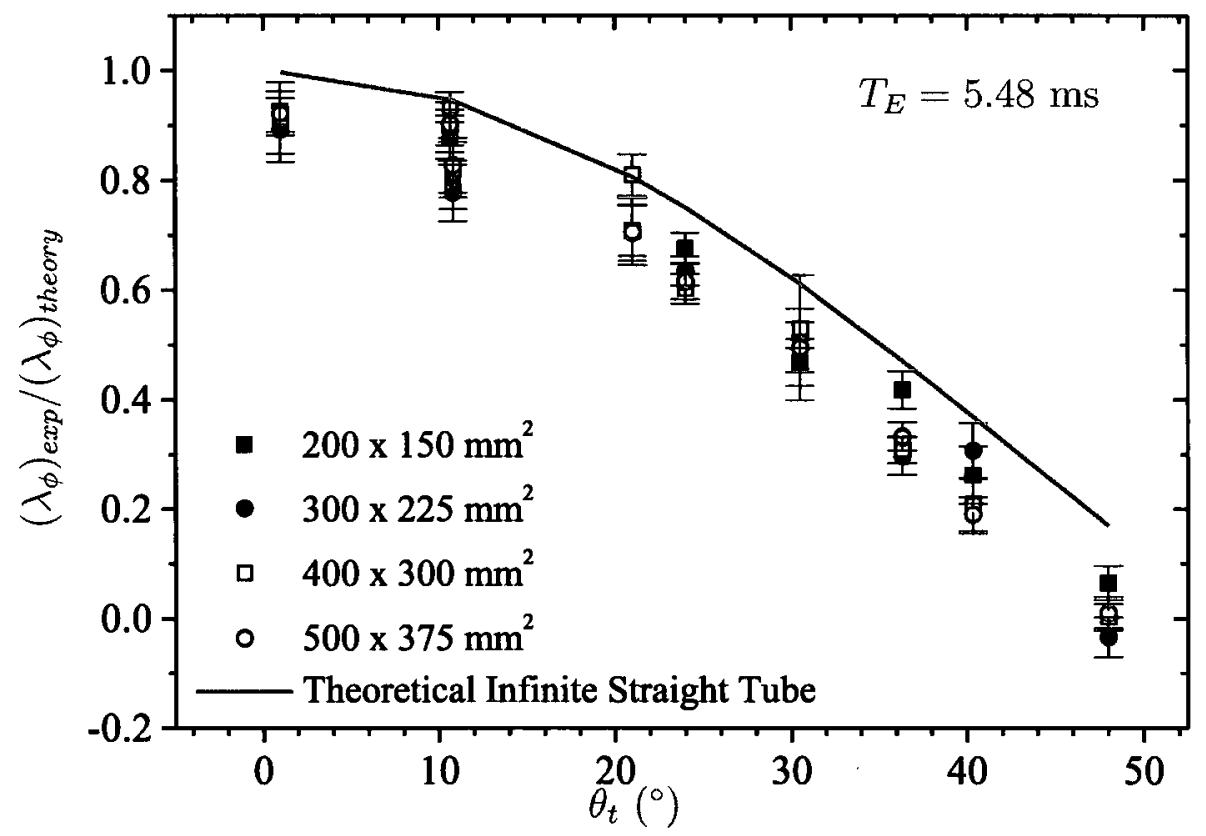

Figure 4.6: $\left(\lambda_{\phi}\right)_{\text {exp }} /\left(\lambda_{\phi}\right)_{\text {theory }}$ as a function of angle, $\theta_{t}$, for several FOVs for a curved tube $\left(T_{E}=5.48 \mathrm{~ms}\right)$. The thick solid line shows the theoretical value calculated for Eq. 2.113. The error bars represent the standard error calculated from the linear regression.

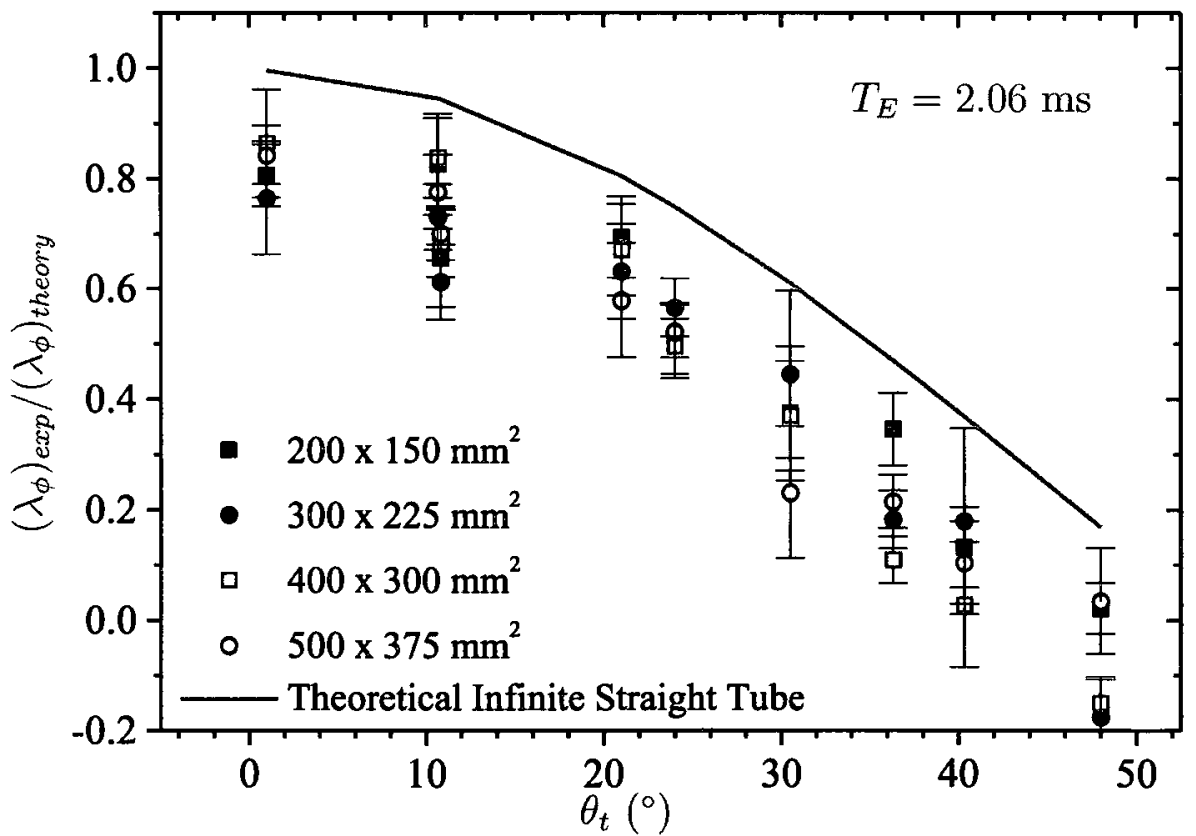

Figure 4.7: $\left(\lambda_{\phi}\right)_{\text {exp }} /\left(\lambda_{\phi}\right)_{t h e o r y}$ as a function of angle, $\theta_{t}$, for several FOVs for a curved tube $\left(T_{E}=2.06 \mathrm{~ms}\right)$. The thick solid line shows the theoretical value calculated for Eq. 2.113. The error bars represent the standard error calculated from the linear regression. 
$\mathrm{mm}$ whereas it was only $0.3 \mathrm{~mm}$ for the thin walled phantom. It was hypothesized that they could be more heavily influenced by signal leakage (see Section 2.8.3). It was impractical to use this material for curved tube experiments since it was not flexible. As described in the Methods section, only a subset of the angles $\left(0^{\circ}, 15^{\circ}\right)$ were studied.

Figs. 4.8 and 4.9 show $\left(\lambda_{\phi}\right)_{\text {exp }}$ normalized to the infinite straight tube theoretical solution for all FOVs and $\theta$ 's. The mean and SD of the percent error in the ratio of the experimental to theoretical $\phi$ to $[\mathrm{CA}]$ slopes were $(-11 \pm 3) \%$ and $(-27 \pm 3) \%$ for $T_{E}$ 's of 5.48 and $2.06 \mathrm{~ms}$, respectively. The precision of these values is comparable to the straight, thick walled phantom results but the mean slope values are lower. The derivation of the mean values from the predicted curve is about the same size as for the curved tube experiments. The COV for $\left[\mathrm{CA}_{1 m M}\right]$ for the thin walled tubes averaged over all FOVs and $\theta$ 's was $(7 \pm 1) \%$ for the $T_{E}$ of $2.06 \mathrm{~ms}$ and was $(2.4 \pm$ $0.3) \%$ for the $T_{E}$ of $5.48 \mathrm{~ms}$. These are consistent with the corresponding straight tube values of $(5 \pm 2) \%$ and $(2.0 \pm 0.5) \%$, respectively.

\subsection{4 $\mathrm{T}_{1}$ Results}

The results in Table 4.5 compare the values of $T_{1}$ calculated using the IR and VFA methods. Figure 4.10 shows that the linear relationship between $1 / T_{1}$ and [CA] is confirmed for both methods and a linear regression for the VFA derived data is also represented. The slope of this linear fit, which is the measured CA relaxivity, is consistent with the literature values of the relaxivity of Gadovist $\mathbb{B}$ ) (within $1 \mathrm{SD}$, percent error $=1.3 \%$ ). This result validates the VFA method for measuring $T_{1}$. 


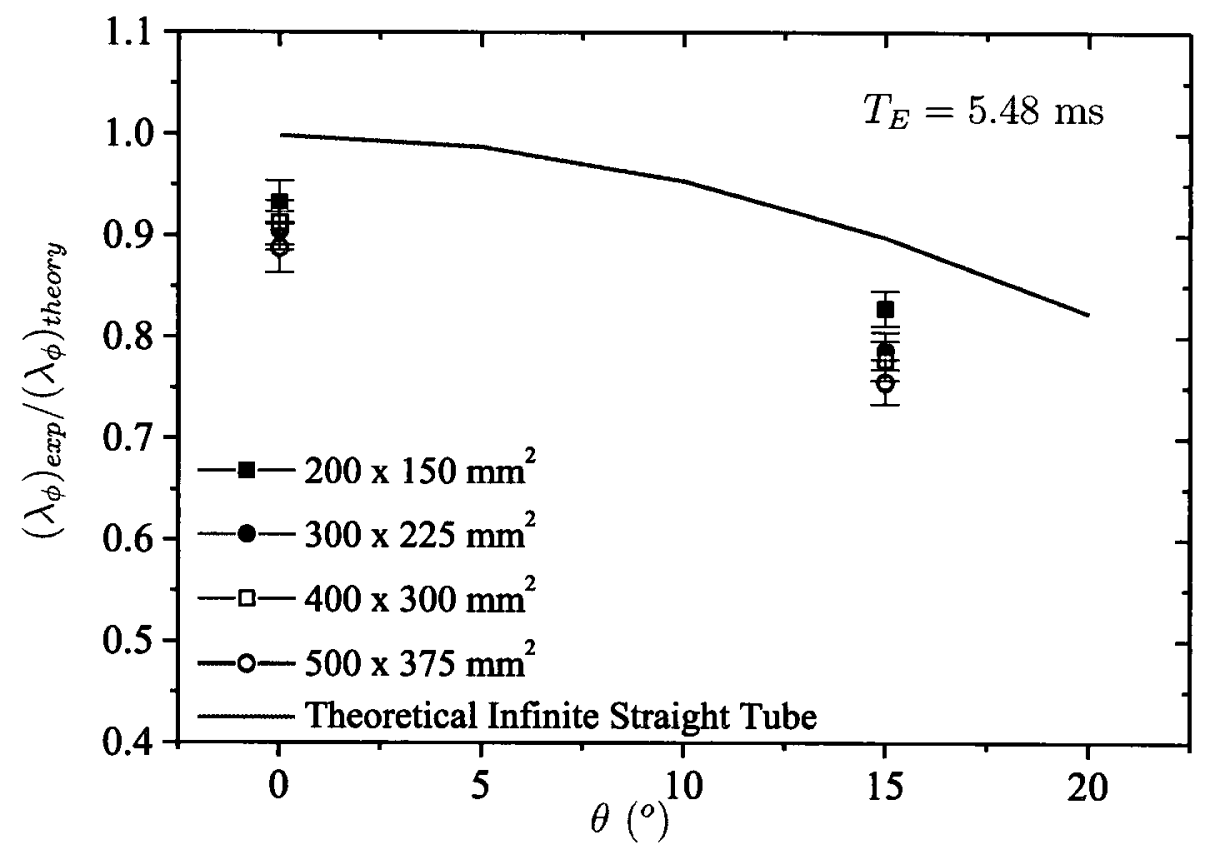

Figure 4.8: $\left(\lambda_{\phi}\right)_{\text {exp }} /\left(\lambda_{\phi}\right)_{\text {theory }}$ as a function of angle, $\theta$, for several FOVs for a thin walled straight tube parallel to $B_{0} \hat{z}$, with $T_{E}=5.48 \mathrm{~ms}$. The thick solid line shows the theoretical calculation from Eq. 2.113. The error bars represent the standard error calculated from the linear regression.

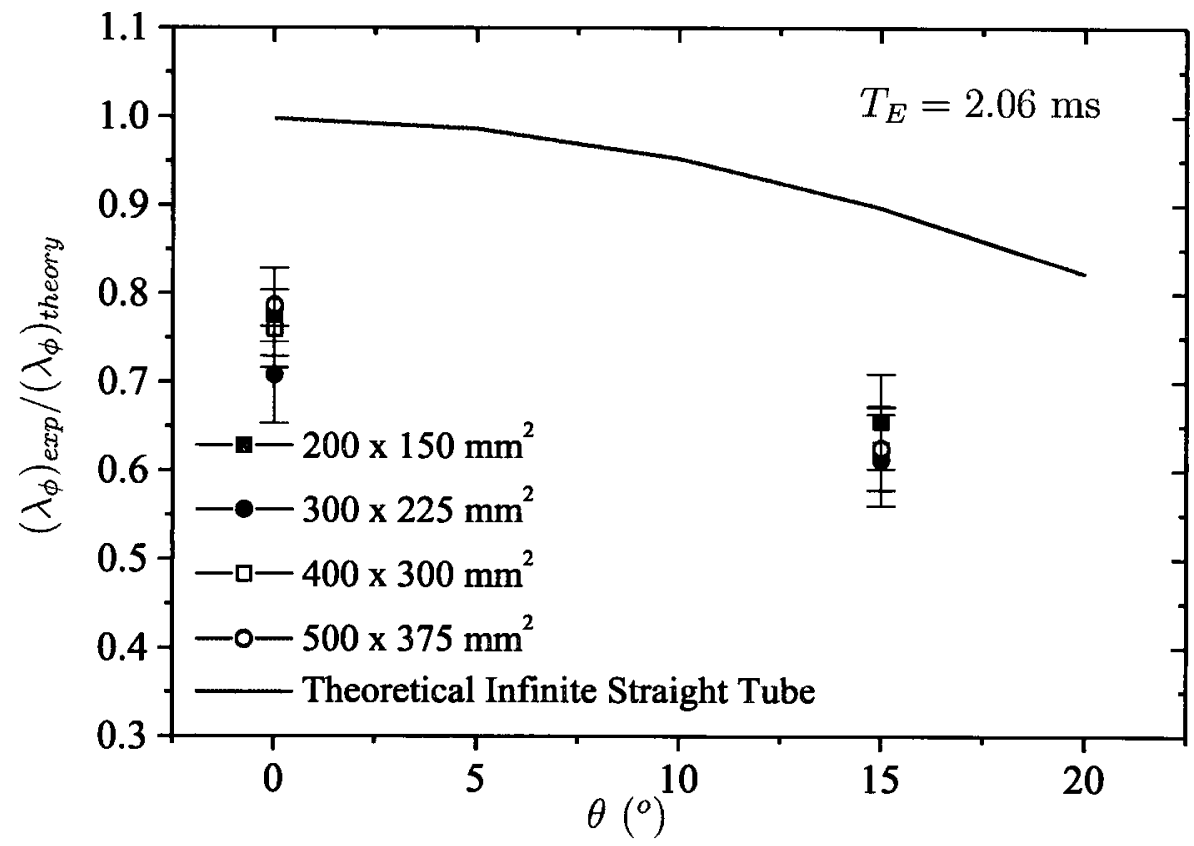

Figure 4.9: $\left(\lambda_{\phi}\right)_{\text {exp }} /\left(\lambda_{\phi}\right)_{\text {theory }}$ as a function of angle, $\theta$, for several FOVs for a thin walled straight tube parallel to $B_{0} \hat{z}$, with $T_{E}=2.06 \mathrm{~ms}$. The thick solid line shows the theoretical calculation from Eq. 2.113. The error bars represent the standard error calculated from the linear regression. 
Table 4.5: Calculated $T_{1}$ results for the blood vessel phantom using the inversion recovery and variable flip angle methods. An assumed $T_{1}$ for water of 4000 ms was used to convert from [CA] to the expected values of $T_{1}$ for Gadovist $\AA$ given in column 2 .

\begin{tabular}{|cccc|}
\hline $\mathrm{C}(\mathrm{mM})$ & $T_{1}(\mathrm{~ms})$ & $T_{1}$ from IR $(\mathrm{ms})$ & $T_{1}$ from VFA $(\mathrm{ms})$ \\
\hline 0.05 & 2062 & $1646 \pm 6$ & $2400 \pm 200$ \\
0.125 & 1194 & $1090 \pm 6$ & $1370 \pm 70$ \\
0.275 & 648 & $653 \pm 2$ & $690 \pm 50$ \\
1 & 202 & $187 \pm 1$ & $192.7 \pm 0.9$ \\
2 & 104 & $118 \pm 4$ & $105.2 \pm 0.7$ \\
3 & 70 & $74.2 \pm 0.3$ & $69.3 \pm 0.7$ \\
4 & 52 & $45.6 \pm 1.1$ & $51.7 \pm 0.5$ \\
6 & 35 & $33 \pm 2$ & $36.0 \pm 0.5$ \\
8 & 26 & $22.6 \pm 0.7$ & $25.7 \pm 0.6$ \\
10 & 21 & $21.7 \pm 0.9$ & $23.4 \pm 0.6$ \\
\hline
\end{tabular}

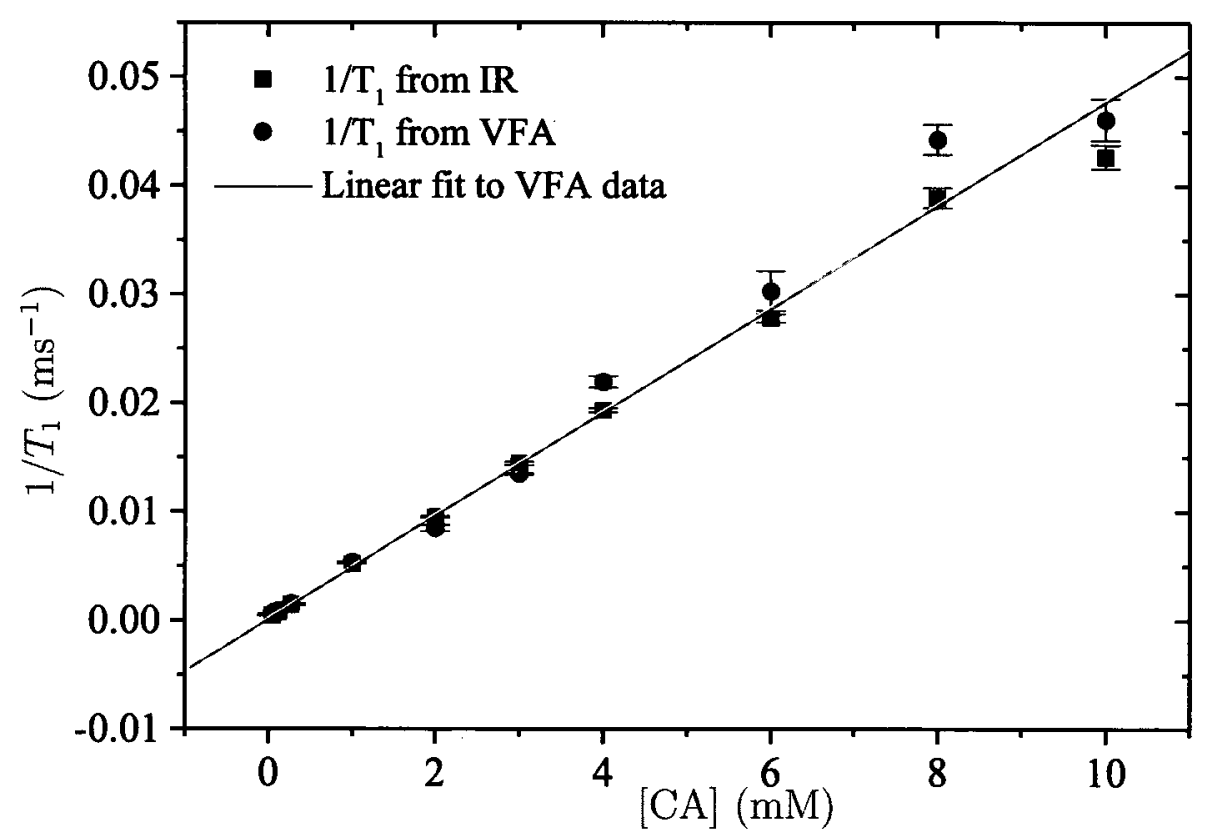

Figure 4.10: $1 / T_{1}$ as a function of $[\mathrm{CA}]$ for the inversion recovery method and variable flip angle method. The linear fit for the VFA method data was $1 / T_{1}=(4.76 \pm$ $0.08) \mathrm{mM}^{-1} \mathrm{~s}^{-1} *[\mathrm{CA}]+(0.28 \pm 0.09) \mathrm{s}^{-1}$ with $\mathrm{R}=0.99874$ and $p<0.0001$. The error bars represent the standard error calculated from the parameter fit. 


\subsection{Discussion}

The goal of this chapter is to show experimentally that the $\Delta \phi$ predicted by Eq. 4.1 is correct for a variety of experimental conditions. Phantoms resembling sections of human arteries and veins were constructed from tubing with known [CA], up to $10 \mathrm{mM}$. The $\Delta \phi$ for these phantoms was then measured. The slope given by Eq. 4.2 depends on $T_{E}, B_{0}$ and the geometry factor. The experiments were repeated for the two $T_{E}$ values used in the clinical studies presented in Chapters 6 and 7 and as a function of $\theta$. All the results presented in this thesis were obtained at $B_{0}=1.5 \mathrm{~T}$ so the dependence on $B_{0}$ was not investigated here. In all cases for $|\theta|<25^{\circ}$, the measured $\phi$ dependence on $[\mathrm{CA}]$ was linear $(\mathrm{R}>0.91)$ and the slope was in reasonable agreement with the value predicted by Eq. 4.2. The individual experiments are discussed in more detail below.

There was also some concern that the effects of partial volume averaging and/or signal leakage (see Section 2.8.2) could have an effect on the $\phi$ versus [CA] conversion relationship as the voxel size increases relative to the size of the vessel lumen. This potential problem was investigated by repeated all of the experiments as a function of resolution by varying the FOV while keeping the matrix size constant for both a thick walled phantom and a thin walled phantom. The $\phi$-to- $[\mathrm{CA}]$ conversion relationship given by Eq. 4.1 was found to be valid for both phantoms and all resolutions studied.

\subsubsection{Geometry Factor, $g(\theta)$, for the Straight Tube Phantom}

The dependence of $\lambda_{\phi}$ on the geometry factor, $g(\theta)$, was initially tested using straight tubes filled with different $[\mathrm{CA}]$ as a function of the orientation of the tube with respect to $\mathbf{B}_{\mathbf{0}}$. The linear relationship between $\phi$ and $[\mathrm{CA}]$ was confirmed for all cases and the measured slope was in agreement with the value predicted by Eq. 4.2 for all $\theta$ to within $\pm 3 \%$. As expected, $\lambda_{\phi}$ decreased significantly as $\theta$ was increased from $0^{\circ}$ to 
$20^{\circ}$. This confirms the expectation that $\theta$ must be accounted for whenever possible. This should not normally be a problem for typical clinical DCE-MRI protocols since, in principle, $\theta$ can be determined from $3 \mathrm{D}$ anatomical images acquired as part of the standard imaging exam. There is a predicted loss of $\mathrm{SNR}_{\Delta \phi}$ as the angle increases but this is not apparent within the precision of the experiments presented here.

The experiments performed on the straight tube phantoms demonstrated that, for $\theta$ values up to $20^{\circ}$, measurements of $\phi(t)$ can be used to determine $C(t)$ without loss of precision relative to the $\theta=0$ case, for the protocol proposed here.

\subsubsection{Geometry Factor, $g(\theta)$, for the Curved Tube Phantom}

Although many veins and arteries of the human body have straight sections (e.g. common carotid artery, descending aorta), others, that may be of interest for DCEMRI measurements, are better approximated as curved tubes (e.g. superior sagittal sinus). Therefore, the experiments performed on the straight tube phantoms were repeated on a curved tube phantom which had a radius of curvature of $45 \mathrm{~mm}$. The orientation of the tube at any location along the tube was taken to be $\theta_{t}$, the tangent to the tube at that location, and this is the angle that was used to calculate the geometry factor for these experiments.

The measured results agreed reasonably well with the values predicted by Eq. 4.2, even though this equation is strictly valid only for infinitely long straight cylinders. In addition the disprepancy between the measured slopes and the theoretical slope for an infinitly long straight tube appears to be a consistent shift for all angles. This suggests that the relative change in measured slope with change in $\theta_{t}$ follows that predicted by the "straight tube approximation". This is a significant result since, even though a theoretical expression for the relationship between $\phi$ and $[\mathrm{CA}]$ is unknown for the curved tube geometry, the straight tube solution can be used to predict the results for a curved tube, when the orientation of the tube is equated with the angle between 
the tangent to the tube at that location and $\mathbf{B}_{\mathbf{0}}$. This "straight tube approximation" will be explored further in Chapter 5 .

When the angle is larger than $30^{\circ}$ the percent error can be $50 \%$ or more, particularly when the $T_{E}$ is small or the FOV is large. As $\theta$ approaches $55^{\circ}$ (corresponding to $\left.3 \cos ^{2} \theta-1=0\right) \lambda_{\phi}$ goes to zero. This means there is a reduction in $\Delta \phi$ sensitivity at these larger angles. Since partial volumes and signal leakage tend to have a stronger effect on the low $[\mathrm{CA}]$ data points compared to the high $[\mathrm{CA}]$ data points, this can cause larger shifts in the $\phi$ at lower [CA] values than at high [CA]. This leads to a deviation in $\lambda_{\phi}$ from the predicted value. These situations should be avoided for this protocol. If the $\phi$ of the background is more similar to the $\phi$ in the vessel at low [CA] it is likely that angles larger than $25^{\circ}$ could be included as well, as the effects of partial volumes and signal leakage would be minimized.

In the case of the curved tubes where $\theta<25^{\circ}$, the mean for percent difference and COV were larger than in the straight tube experiment. There are several possible reasons for this. It could be that the straight tube approximation introduced some error. This will be investigated through simulation in Chapter 5. It is possible that the angles were not measured with sufficient accuracy. It is also possible that the setup of the curved tube experiment resulted in a more significant signal leakage effect. From an inspection of the phase images it appears that the apparatus used to keep the phantom stabilized in the curved shape causes a variation in the $\phi$ of the surrounding water; this could also have contributed to the observed deviation. This apparatus would not be a factor for in vivo measurements.

\subsubsection{Echo Time}

The expected linear behaviour of the $\phi$ versus $[\mathrm{CA}]$ plots was obtained for both echo times studied and the slope agreed well with the value predicted by Eq. 4.2 for the straight tube and the curved tube experiments. 
For DCE-MRI measurements, in general, it would appear that more accurate results should be obtained when longer echo times are used since $\mathrm{SNR}_{\phi}$ increases with $T_{E}$, as long as the $T_{E}$ is not so large that phase wrap or loss of $|S|$ due to $T_{2}^{*}$ become a problem. The use of longer $T_{E}$ 's, however, reduces the time resolution which, in turn, decreases the accuracy of the AIF measurements, counteracting the gain obtained from the higher $\mathrm{SNR}_{\phi}$. Thus, a balance between these two effects must be reached when planning the $T_{E}$ value to be used for the DCE-MRI protocol. For $T_{1}$-weighted measurements such as are employed for DCE-MRI experiments, $T_{E}$ is typically chosen to be $3-4 \mathrm{~ms}$. The $T_{E}$ 's used for the work presented in this chapter were $2.06 \mathrm{~ms}$ and $5.48 \mathrm{~ms}$. The predicted gain in $\mathrm{SNR}_{\phi}$ and accuracy with increasing $T_{E}$ was observed for $T_{E}=5.48 \mathrm{~ms}$ relative to $T_{E}=2.06 \mathrm{~ms}$, however, gain was not large. The COVs for the $\phi$ versus [CA] data for $T_{E}=2.06 \mathrm{~ms}$ and $T_{E}=5.48 \mathrm{~ms}$ were, on average, $(5 \pm 2) \%$ and $(2.0 \pm 0.5) \%$, respectively, for the straight tube experiment and $(11 \pm 3) \%$ and $(5.4 \pm 1.4) \%$, respectively, for the curved tube experiment. Although the increased accuracy for the longer $T_{E}$ is notable, the error for the shorter $T_{E}$ in these phantom experiments is still small enough to justify its use in a DCE-MRI protocol where increased time resolution for the AIF measurements is desirable. This is investigated again in Chapter 6.

\subsubsection{Field of View}

To get reliable perfusion parameters from DCE-MRI experiments it is important to measure the AIF accurately. Unfortunately, the arteries are relatively small and often the AIF has to be determined from only a few voxels. Furthermore, when so few voxels are involved, partial volume and signal leakage effects can incorporate signal that originates outside of the vessel into the signal used to determine the AIF. This is clearly undesirable. To investigate the importance of this effect for DCEMRI measurements performed using $\phi$, the phantom experiments reported here were 
repeated as a function of resolution by varying the FOV for a constant matrix size of $48 \times 128$. The number of voxels contained completely inside the phantom tube, assuming sharp voxel boundaries, depended to some extent on the position of the voxels relative to the tube. For the best case scenario, where the center of the tube cavity corresponded with the center of the voxel, the number of whole voxels contained inside the tube cavity for the resolutions studied here correspond to $1 \times 5,1 \times 3,1 \times$ 1 and $1 \times 1$ with differing amounts of partial volume averaging in each case.

One deficiency of the FOV study discussed above is that the phantom used had thick walls. Since the walls contribute no MR signal these phantoms may not be a good representation of the situation with blood vessels in vivo in terms of partial volume averaging effects. The straight tube study was therefore repeated with thin walled tubing with a wall thickness of $0.3 \mathrm{~mm}$. The COV for the thin walled experiments was $(7 \pm 1) \%$ for the $T_{E}$ of $2.06 \mathrm{~ms}$ and was $(2.4 \pm 0.3) \%$ for the $T_{E}$ of $5.48 \mathrm{~ms}$ which is consistent with the thick straight tube values of $(5 \pm 2) \%$ and $(2.0 \pm 0.5) \%$, respectively. These results suggest that there is no increase in variation across FOV when the wall thickness is reduced.

The effect of partial volume and signal leakage on the data presented here was perhaps larger than would be expected in vivo. The background water was doped with CA to give it a $T_{1}$ similar to tissue. The water in the tube however was not doped so the $|S|$ inside the tube, at low [CA] data points, was lower that the $|S|$ of surrounding signal. This makes these low [CA] data more susceptible to signal leakage and partial volume contributions from these surrounding vectors. In vivo it is not likely that the $|S|$ inside the vessel will be as different from the surrounding $|S|$ as in the phantom tube study because the $T_{1}$ of blood is lower than that of water. In fact, in cases were there is inflow of blood into the imaging volume, the $|S|$ inside the vessel can be brighter than the surrounding tissue, even at low [CA]. This experimental design allowed for the comparison of the partial volume and signal leakage effects at different 
FOV's, the overall deviation from theory is perhaps greater than would be seen in vivo.

For the straight tube data, a statistically significant variation in the slope was found as a function of FOV. Although the effect was statistically significant, it was relatively small, as seen in Table 4.4. This effect was not seen in the curved tube experiments. The larger COV observed in the curved tube data suggests that the error on the slope may have eclipsed the FOV effect in the two-factor ANOVA test. Using the same simulation method described in [31], and briefly in Section 2.8.3, it was estimated that the effect should be less than $12 \%$. It is of course ideal to use a sufficient resolution such that there are several pixels within the vessel. However, when optimizing between spatial resolution and time resolution this is not always possible. It can be seen from these results that measurements obtained from vessels with a radius on the order of one pixel can still be reliable, provided the echo time is long enough.

\subsubsection{Over and Underestimation}

It may be noted that most of the data points in Fig. 4.4 lie above the theoretical curve, whereas those in Fig. 4.6 and Fig. 4.8 lie below the line. As discussed in Section 2.8.3 an underestimation or overestimation of the $[\mathrm{CA}]$ is possible depending on the $\phi$ of the surrounding material. This variation may be due to differences in the experimental setup between the straight and curved tubes. This difference illustrates that one cannot assume an under- or overestimation without further information about the surrounding material. One can imagine a correction scheme involving the $\phi$ and $|S|$ of the signal from the surrounding material, which could improve the results. This approach could be the subject of future work. Partial volume correction schemes have been applied to the $T_{2}^{*}$-weighted dynamic CA injection case where the $|S|$ signal decreases with $[\mathrm{CA}]$, instead of increasing and where the $\phi$ accumulation is 
considerably greater owing to the longer $T_{E}[44,45,46]$.

\subsection{Conclusions}

These phantom experiments provide a validation of the proposed technique and provide a foundation for the application of this technique to in vivo data, which will be explored in Chapter 6 and Chapter 7. The phantom experimental results show that the technique is reliable to within $11 \%$ for $T_{E}=5.48 \mathrm{~ms}$ for angles less than $25^{\circ}$. The expected relationship between precision and $T_{E}$ was also confirmed. This increase in precision with increased echo time must be weighted against time resolution considerations. It was noted that angle is the main source of error and should be accounted for whenever possible. Once the angle was considered it did not contribute additional error up to $25^{\circ}$ at which point the SNR in this experiment became insufficient. It was also concluded that the pixel size relative to the vessel size affects the results, and thus larger vessels are preferable. When designing a DCE-MRI protocol which includes phase measurements of the vessel, some care must be taken to ensure that an appropriate vessel will be available. The straight tube approximation also appears successful and will be investigated from a theoretical point of view in the next chapter. In addition the proceeding chapter explores a more efficient method for incorporating the vessel geometry. 


\section{Chapter 5}

\section{Calculating the Geometry Factor}

\section{$5.1 \quad$ Introduction}

As demonstrated in the previous chapter, when using the MRI signal phase accumulation, $\Delta \phi$ to determine the concentration of paramagnetic contrast agent $([\mathrm{CA}])$ inside a vessel, the geometry of the vessel must be considered. One cannot simply apply the $\Delta \phi$-to- $[\mathrm{CA}]$ conversion valid for an infinitely long cylinder parallel to the main magnetic field, as is often done in the literature. Thus, it is necessary to incorporate a geometry factor (defined in Section 2.8.2) into the calculation of the magnetic field shift induced by the CA. This will be the focus of the present chapter. In some simple cases, the geometry factor can be found analytically; this is true in the case of an infinitely long cylinder at an angle $\theta$ with respect to $\mathbf{B}_{0}$. The theory describing this scenario was presented in Section 2.7.2. In the case of more complex geometry, a value can be obtained using computational methods like the one proposed by Marques and Bowtell [25]. The derivation of this method was presented in Section 2.7.3.

In the DCE-MRI technique proposed in this thesis, evolution of the $\phi$ from blood vessels, which is affected by local changes of magnetic susceptibility induced by the presence of $\mathrm{CA}$ in those vessels; is used to determine the $[\mathrm{CA}]$ at that location as a function of time. Since these vessels are essentially cylinders with curved geometries, 
a reasonable value for the geometry factor can be obtained by introducing the hypothesis that, for any specific location along this curved cylinder, the vessel can be treated as an infinitely long straight vessel oriented at an angle equal to the tangent to the curve at that point. This approach is used effectively in Chapters 4 and 6 of this thesis and is validated in the present chapter.

There are three main objectives of the work presented in this chapter. The first is to implement a method to estimate a geometry factor for an arbitrary magnetic susceptibility distribution and use it to correct a phase map. The next is to use this method to assess the validity of the straight tube approximation. The final objective is to assess the usefulness of using magnitude MRI images to create a susceptibility map that can be used to calculate the geometry factor inside the superior sagittal sinus (SSS).

\subsection{Method}

It was shown in Section 2.8.2 that the phase change, $\Delta \phi(t)$, caused by the presence of a CA of concentration $C(t)$ for a general vessel geometry is given by

$$
C(t)=\frac{3 \Delta \phi(t)}{\gamma T_{E} B_{0} \chi_{m}} g(\mathbf{r}),
$$

where $g(\mathbf{r})$ is a generalized geometry factor. To evaluate $g(\mathbf{r})$ the method proposed by Marques and Bowtell [25] and discussed in Section 2.7.3 can be used. It will be assumed that the position of the vessels in the volume of interest can be determined from MRI images using, for example, a thresholding algorithm. A susceptibility map can then be generated for this volume by setting the interior of these vessels to $\chi=\chi_{0}+C \chi_{m}$ for some assumed value of $[\mathrm{CA}]$. With this susceptibility map as input, the method of Marques and Bowtell can be used to calculate the change in magnetic 
field and the corresponding phase shift, $\Delta \phi_{s i m}$, caused by this distribution of CA. Thus $g(\mathbf{r})$ can be calculated from

$$
g(\mathbf{r})=\frac{\gamma T_{E} B_{0} \chi_{m}}{3} \frac{C}{\Delta \phi_{s i m}}
$$

This can also be written (see Eq. 2.114) as:

$$
g(\mathbf{r})=\frac{\Delta \phi_{r e f}}{\Delta \phi_{s i m}}
$$

where $\Delta \phi_{\text {ref }}$ is the phase accumulation for an infinitely long cylinder at $\theta=0$ for the same [CA], $B_{0}$ and $T_{E}$ as were used in the simulation.

A combination of simulations, phantoms and clinical data were used to assess the validity and usefulness of the proposed computational method. First the implementation of the Marques and Bowtell [25] technique is given. The next three sections explain how the test data was generated using computer simulated blood vessels, a blood vessel phantom and finally clinical data. The phantom and clinical data analysis technique used in this chapter are closely related to the methods applied in other chapters, and references are given to the applicable section, where appropriate, to avoid repetition.

\subsubsection{Dipole Approximation Method}

The theoretical presentation of the Marques and Bowtell method [25] was given in Section 2.7.3. Here the specifics of its implementation are addressed. This program was implemented in Interactive Data Language, or IDL®), versions 6.2-7.0, (ITT Visual Information Solutions, Boulder, Colorado); the code is given in Appendix B.

The first step in the process is to generate a 3D matrix of magnetic susceptibility, $\chi(\mathbf{r})$. If a susceptibility map is available, this can be used. If not, one can be created 
from either a simulated volume or from an MRI image where the pixel values are known to be proportional to susceptibility. Next, the $z$ component of magnetization is calculated, under the assumption that $|\chi(\mathbf{r})|<<1$ for all $\mathbf{r}$ in the matrix. As discussed in Section 2.7.1, this is an appropriate assumption for clinical MRI. Applying this assumption to Eq. 2.88 gives

$$
M_{z}(\mathbf{r})=\frac{B_{0}}{\mu_{0}} \chi(\mathbf{r})
$$

Next, the Fourier transform of Eq. 5.4 is calculated. This gives

$$
\tilde{M}_{z}(\mathbf{k})=\mathcal{F}_{3 D}\left(M_{z}(\mathbf{r})\right)
$$

where $\tilde{M}$ indicates the Fourier transform of $M$ and $\mathcal{F}_{3 D}$ represents the 3D Fourier transform. From this the magnetic field perturbation can be computed as

$$
B_{d}(\mathbf{r})=\mathcal{F}_{3 D}^{-1}\left(-\frac{\mu_{0}}{3}\left(3 \cos ^{2} \beta-1\right) \tilde{M}_{z}(\mathbf{k})\right)
$$

which is the final result presented in Section 2.7.3 (Eq. 2.106). Note the $z$ subscript has been removed for simplicity, for all simulations presented here the main magnetic field was parallel with the $z$-axis.

This equation is not well defined when $\mathbf{k}=\mathbf{0}$. To deal with this, Marques and Bowtell [25] assume that the object is surrounded by a region of infinite extent whose susceptibility is equal to $\chi_{e}$. This means that

$$
\tilde{B}_{d}(\mathbf{k}=\mathbf{0})=+\frac{B_{0} \chi_{e}}{3}
$$

where $\tilde{B}_{d}$ is the Fourier transform of the magnetic field perturbation defined above.

When applying this technique, the matrix size must be "significantly larger than the spatial extent of the ROI" [25]. The implications of this statement are explored in 
Section 5.3.1, although every effort was made to use the largest matrix size practically possible for this work.

The case when the voxels of the input matrix do not have a symmetric resolution is not specifically addressed by Marques and Bowtell [25]. In this case, the calculation of $\mathbf{k}$ should be scaled by the voxel dimension of $\mathrm{k}$-space, for each dimension as follows:

$$
k_{i}=\left\{\begin{array}{lll}
\frac{I}{N_{i} \Delta x_{i}}, & 0 \leq I \leq \frac{N_{i}-1}{2}, & I \in Z \\
\frac{I-N_{i}}{N_{i} \Delta x_{i}}, & \frac{N_{i}-1}{2}<I \leq N_{i}, & I \in Z
\end{array}\right.
$$

where $I$ is the index in the $i^{\text {th }}$ dimension, $N_{i}$ is the number of pixels in the matrix along the $i^{\text {th }}$ dimension, $\Delta x_{i}$ is the length of one pixel in the $i^{\text {th }}$ dimension.

The computations were done using the IDL data type "complex" which consists of a pair of single-precision floating-point numbers. Each is a 32 -bit number in the range of $\pm 10^{38}$, with seven decimal places of significance. In all cases eight significant figures were kept for calculations, but the results were are rounded to six significant figures when quoting $B_{d}(\mathbf{r})$.

\subsubsection{Simulated Blood Vessels}

To simulate a blood vessel, a 3D matrix ranging up to $512^{3}$ elements was generated containing a cylinder to represent the vessel. The voxels outside the cylinder were assumed to be water and they were given a value of $\chi_{0}=-1 \times 10^{-5}$, the magnetic susceptibility of water [25]. The voxels inside the cylinder were given a value of $\chi_{m} C+\chi_{0}$, where $\chi_{m}$ is the mass magnetic susceptibility of the contrast agent (CA) and $C$ is the concentration of CA, [CA] (see Section 2.8.2). It was assumed that the walls of the vessel were infinitely thin and that they did not cut through any volume elements; voxels were either entirely inside the vessel or entirely outside the vessel.

The simulated vessel shapes include infinite and finite cylinders at various angles 
Table 5.1: Polynomial fits used to generate simulated vessels.

\begin{tabular}{|cc|}
\hline Study ID & $2^{\text {nd }}$ Order Polynomial Fit \\
\hline P006-S001 & $r=0.014 z^{2}-0.6407 z+1.4847$ \\
P012-S001 & $r=0.0066 z^{2}-0.0292 z+0.4244$ \\
P017-S001 & $r=0.0055 z^{2}-0.2974 z-0.0795$ \\
\hline
\end{tabular}

with respect to the magnetic field, tori with various radii of curvature and curves following a $2^{\text {nd }}$ order polynomial. If the voxel fell inside or on the boundary, it was included in the vessel volume.

In addition to these regular volumes, simulated volumes based on the geometry of the SSS of several patients were also generated. Since the object of this simulation was to assess the validity of the straight tube approximation, patients who appeared to have a particularly curved SSS near the regions of interest in the DCE-MRI study were selected (P006-S001, P012-S001 and P017-S001). The polynomial fit was determined by drawing a one- or two-pixel ROI at the most central pixel in the SSS for each slice of a post contrast 3D image, acquired as part of the brain protocol (see Section 6.2). The image position and orientation, provided in the image file header (DICOM format), were used to map the location of the ROI onto the patient/magnet coordinate system. Next, a $2^{\text {nd }}$ order polynomial fit was applied to the data. The polynomial fits used are given in Table 5.1. The constant term in the polynomial fit is not important and is chosen in the simulation to place the portion of the curve near the ROI in the center of the matrix. To generate the 3D shape, a sphere of radius equal to that of the vessel, which was set to 16-pixels, was drawn at discrete points along the curve defined by the polynomial. For a $512^{3}$ matrix, 5000 spheres gave a sufficiently smooth curve as determined by eye. 


\subsubsection{Phantom Tubes}

Plastic tubing with an inner diameter of $9 \mathrm{~mm}$ and an outer diameter of $13 \mathrm{~mm}$ (the same dimensions described in Chapter 4) was arranged in a complex curved geometry, as shown in Fig. 5.1. The tubing was imaged for the case of $1 \mathrm{mM}$ and $10 \mathrm{mM}$ aqueous solutions of Gadovist@ (Bayer Healthcare) at room temperature. In order to avoid signal leakage, as described in Section 2.8.3, the external medium surrounding the tubing was air.

The phantom was imaged on the Siemens Symphony TIM 1.5 T MRI machine (Siemens Medical Solutions, Erlangen, Germany) at the Ottawa Hospital, General Campus using a 3D SPGR sequence with $T_{E}$ 's of 4 and $8 \mathrm{~ms}$, a $T_{R}$ of $12 \mathrm{~ms}$, a flip angle of $20^{\circ}$, and NEX $=2$. The matrix size was $(256 \times 256 \times 192)$ with a resolution of $(1.5 \times 1.5 \times 1.5) \mathrm{mm}^{3}$. The head coil and neck coil were used. This coil combination was treated by the scanner as a phased array coil and $\phi$ was, therefore, calculated automatically by the scanner software, version B15. The resolution was sufficient that zero filling was not necessary, see Section 4.2.3. The $\Delta \phi$ between the $\phi$ maps measured at $1 \mathrm{mM}$ and $10 \mathrm{mM}$ of $\mathrm{CA}$ was calculated on a pixel-by-pixel basis, taking into account phase wrapping (see Section 4.2.3).

A time of 30 minutes elapsed between the acquisition of the $1 \mathrm{mM}$ and $10 \mathrm{mM}$ datasets since the phantom needed to be thoroughly flushed between trials to ensure accurate $[\mathrm{CA}]$ values. For this reason a drift over time is present in the phase data. This drift was removed from the data before comparison with the simulated data. This was done by measuring the phase accumulation in the parallel and perpendicular sections and comparing them to the theoretical values predicted by Eq. 2.113. Then the average of these two phase shifts was applied to all pixels in the dataset. A drift of $-0.173 \mathrm{rad}$ was observed between the two acquisitions. In practice, the drift can be determined by measuring the phase accumulation in the background water, if it 

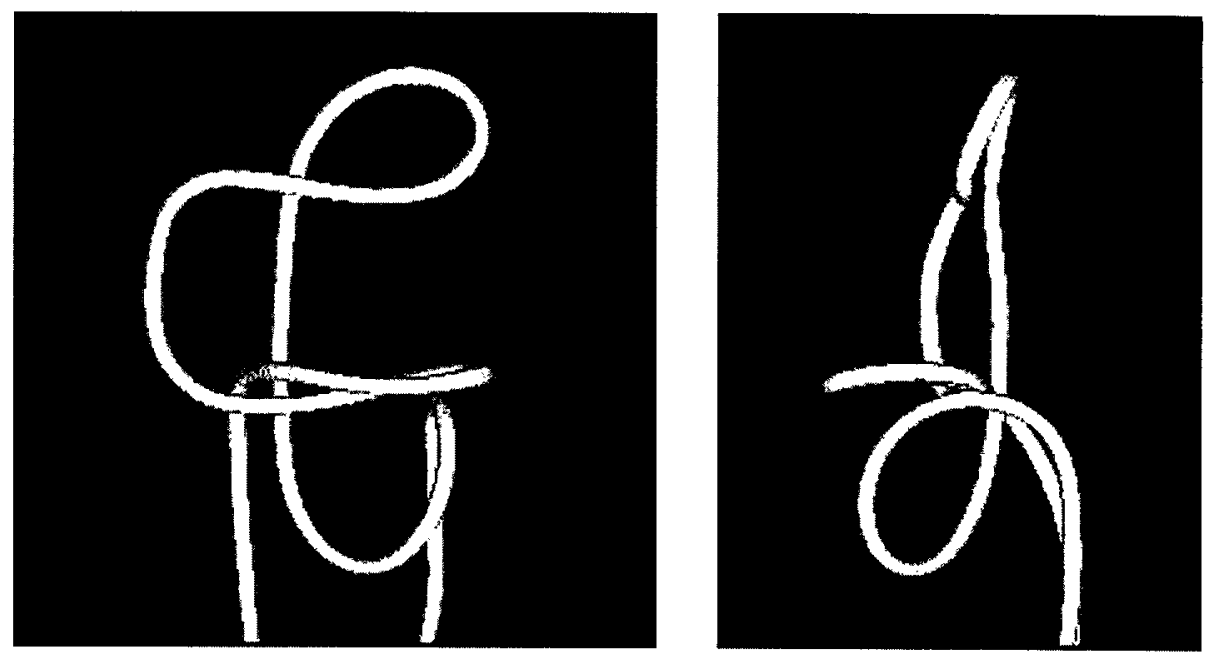

Figure 5.1: $3 \mathrm{D}$ rendering of the plastic tubing used to simulate a blood vessel, calculated from a 3D magnitude image.

is present. In a clinical setting, the exam time is short enough that the amount of phase drifting is minimal and should not be a concern.

\subsubsection{Clinical Cases}

Clinical examples were used to assess the usefulness of segmenting out the SSS on a magnitude MRI image to generate a susceptibility map.

The images were taken as part of the routime clinical exam of the notionts reorn"ted for the brain tumour study described in Chapter 6. The images used were obtained via a sagittal acquisition using a $3 \mathrm{D}$ SPGR sequence with a $T_{E}$ of $2.71 \mathrm{~ms}$, a $T_{R}$ of $6.73 \mathrm{~ms}$ and a flip angle of $15^{\circ}$. The matrix size was (256 x $\left.256 \times 144\right)$ with a resolution of $(1 \times 1 \times 1.5) \mathrm{mm}^{3}, \mathrm{NEX}=1$.

A region growing algorithm was applied to the images to segment out the sagittal sinus from the rest of the image. This was done using Region Growing $3 D$ Segmentation available for ImageJ (National Institute of Health, USA). A 2D representation of a $3 \mathrm{D}$ rendering of an example sagittal sinus generated from clinical data is presented in Fig. 5.2. The data was resampled from $256 \times 256 \times 144$ to $512 \times 512 \times 288$ using IDL's rebin function which uses bilinear interpolation. Next a nearest neighbour 


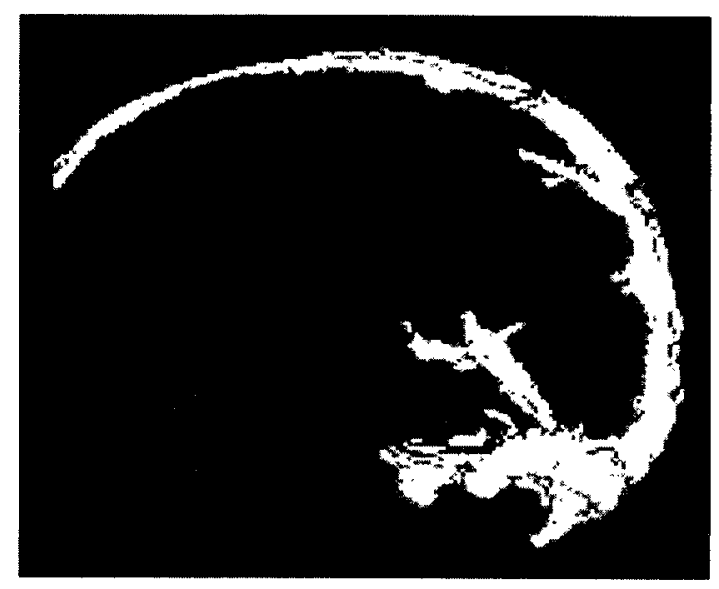

Figure 5.2: 2D representation of a 3D rendering of some of the draining veins of the brain segmented using Region Growing $3 D$ Segmentation (ImageJ). The upper portion is the SSS

averaging smoothing algorithm was applied, as described in Section 5.3.1. The data was then rescaled to have a susceptibility value of $\chi_{m} C+\chi_{0}$ inside the vessel and a value of $\chi_{0}$ outside the vessel.

A set of transverse images was also acquired as part of the brain tumour study protocol discussed in Chapter 6. These four slices were located at the level of the tumour and were part of the dynamic study used to track the bolus of CA as it travelled through the tumour. The location of the transverse DCE-MRI slices in the sagittal image was determined using the image position and image orientation specified in the DICOM image header in fields $(0020,0032)$ and $(0020,0037)$, respectively. This information allowed a selected pixel in a transverse image to be mapped to a pixel in the sagittal image, taking into account the different pixel resolutions. It was assumed that the patient did not move in the time between the dynamic series and the $3 \mathrm{D}$ sagittal image. 


\section{$5.3 \quad$ Results}

The results of the geometry factor calculations are presented here. First, the dipole approximation method proposed by Marques and Bowtell [25] is assessed for application to DCE-MRI using simulated volumes. These results are presented in Section 5.3.1. Next, this method is used for a complex phantom with known [CA], in Section 5.3.2. The validity of the straight tube approximation is assessed in Section 5.3.3, and finally, the dipole approximation method is applied to clinical data in Section 5.3.4.

\subsubsection{Validation of the Dipole Approximation Method on an Infinite Cylinder}

The results presented in this section all involve simulations of an infinitely long cylinder containing a given $[\mathrm{CA}]$ subjected to a $1.5 \mathrm{~T}$ external magnetic field. Unless otherwise stated in the subsection, the matrix size was $512^{3}$, the radius of the cylinder was 16 pixels, and the $[\mathrm{CA}]$ inside the vessels was $1 \mathrm{mM}$. The angle, $\theta$, defining the orientation of the cylinder was measured from the positive $z$ axis to the positive $x$ axis. Throughout this chapter simulations of "infinitely" long cylinders are referred to. This is clearly not an accurate description since a finite matrix size was used for the simulations. However, since no boundary effects were incorporated into the simulations at the ends of the cylinders and since the results compare favourably with theoretical predictions for infinitely long cylinders, it was felt that the use of this terminology was appropriate.

The analysis of these simulated vessels is presented either as a set of 3 orthogonal profiles through a given pixel or as the value at the center of the vessel. The discrepancy between the theoretical solutions given by Eqs. 2.101 and 2.102 (see Section 2.7.2) and simulated $B_{d}(\mathbf{r})$ results computed using the Fourier transform approach 
given by Marques and Bowtell [25] is given in terms of percent error with the analytical solution considered to be the correct result. The error in the $[\mathrm{CA}]$ predicted by the simulation, for $1 \mathrm{mM}$, is also given. Representing the discrepancies in this form allows for easy comparison to other discussions in this work, since accurate [CA] values are the ultimate goal.

\section{Orientation}

First, an infinite cylinder was rotated in the matrix. Figure 5.3 shows the $B_{d}(\mathbf{r})$ profiles of an infinite cylinder at $0^{\circ}, 45^{\circ}$ and $90^{\circ}$ with respect to $B_{0} \hat{z}$. Profiles for both the theoretical and simulated results are given along the $x, y$ and $z$ axes. Each profile is for a single pixel with the center of the tube considered to be the origin. In general the agreement is extremely good. It is clear that most of the discrepancy arises at the interface between the cylinder and the background whereas in the center they match quite well. This is as is expected for a Fourier transform method with finite sampling. For this reason the remaining results are for the center value only. Note that the "wings" on the sides of some of these profiles are a manifestation of the $r^{-2}$ dependence of $B_{d}(\rho>a)$ (see Eq. 2.101) and are expected. 


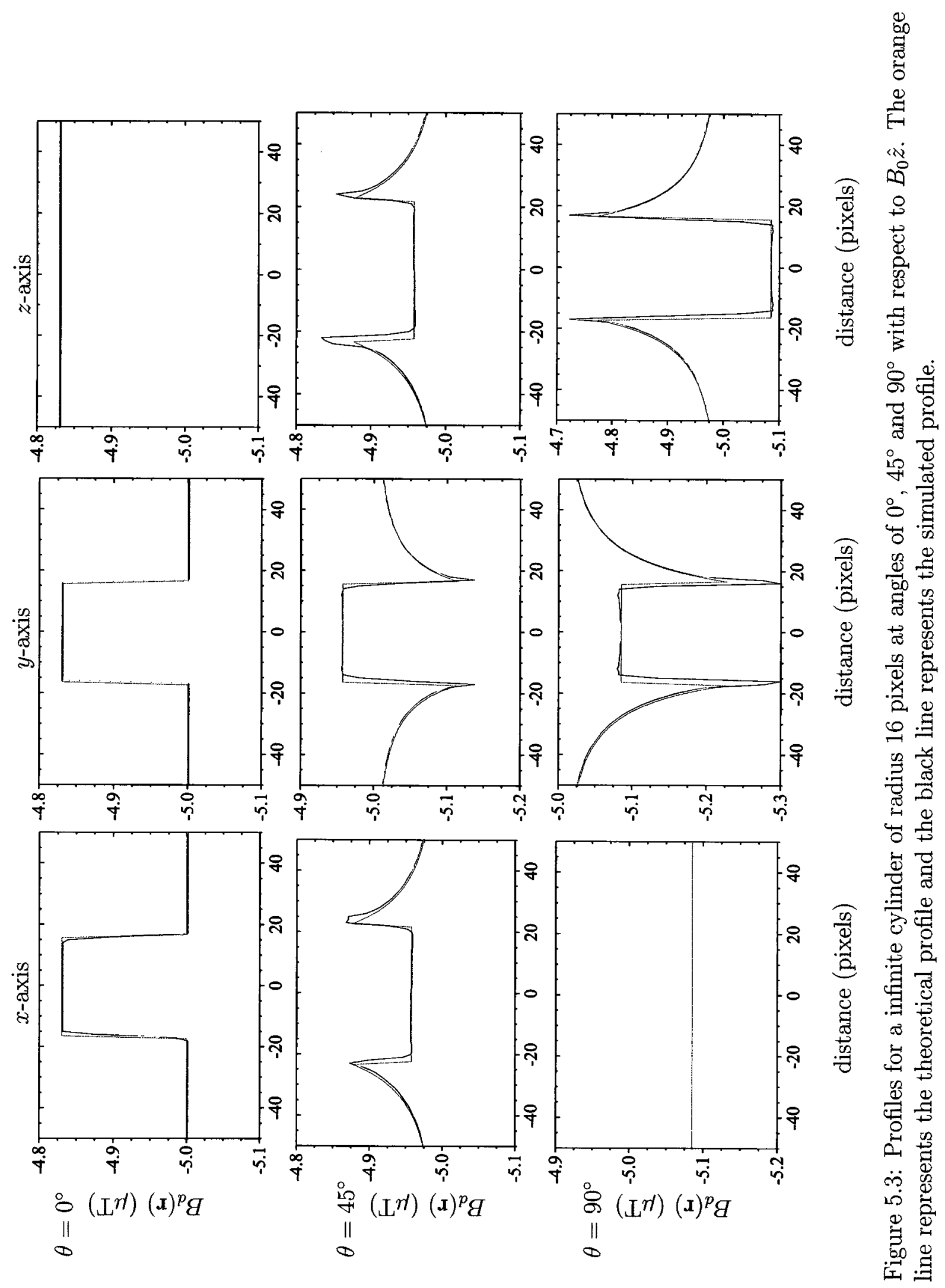


Table 5.2: Simulation results for $B_{d}(\mathbf{r})$ and $C$ for an infinitely long cylinder at various angles. The cylinder was filled with a $1 \mathrm{mM}$ solution of CA and was surrounded by water.

\begin{tabular}{|cccc|}
\hline$\theta\left(^{\circ}\right)$ & $B_{d}(\mathbf{r})_{\text {theory }}(\mu \mathrm{T})$ & $\%$ Error for $B_{d}(\mathbf{r})$ & $\Delta C(\mathrm{mM})$ \\
\hline 0 & -4.83000 & 0.0108 & 0.0031 \\
10 & -4.83769 & 0.0082 & 0.0025 \\
20 & -4.85983 & 0.0071 & 0.0025 \\
30 & -4.89375 & 0.0012 & 0.0005 \\
40 & -4.93536 & -0.0015 & -0.0011 \\
45 & -4.95750 & -0.0011 & -0.0013 \\
50 & -4.97964 & -0.0004 & -0.0010 \\
60 & -5.02125 & -0.0009 & 0.0022 \\
70 & -5.05517 & -0.0070 & 0.0064 \\
80 & -5.07731 & -0.0024 & 0.0015 \\
90 & -5.08500 & -0.0051 & 0.0031 \\
\hline
\end{tabular}

Table 5.2 compares the theoretical and simulated values of $B_{d}(\mathbf{r})$ for the center of the cylinder when it is rotated by an angle $\theta$ with respect to $\mathbf{B}_{\mathbf{0}}$. It can be seen from this table that the discrepancies are small. The $\Delta C$ values are much smaller than the error in [CA] caused by noise in the $\phi$ image which is expected to be on the order of $0.01 \mathrm{mM}-0.1 \mathrm{mM}$ depending on the experimental parameter settings. There does not appear to be a distinct trend in the variation of the error as a function of angle, although the smallest errors were found for $30^{\circ}<\theta<60^{\circ}$.

\section{Size of the Cylinder}

To assess the reliability of the simulation results when a greater proportion of the matrix is occupied by the cylinder, the radius of the cylinder was varied. An infinitely long cylinder with a radius ranging from 16 to 256 pixels was placed in a matrix of $512^{3}$ pixels. Thus, the diameter of the cylinder ranged from $6.25 \%$ to $100 \%$ of the 
Table 5.3: Simulation results for $B_{d}(\mathbf{r})$ and $C$ for infinitely long cylinders of various radii for $\theta=0^{\circ}$ and $45^{\circ}$ with respect to $B_{0} \hat{z}$. The cylinder was filled with a $1 \mathrm{mM}$ solution of CA and was surrounded by water.

\begin{tabular}{|ccc|}
\hline Cylinder Radius (pixels) & $\%$ Error for $B_{d}(\mathbf{r})$ & $\Delta C(\mathrm{mM})$ \\
\hline Case 1: $\theta=0^{\circ}$ & $B_{d}(\mathbf{r})_{\text {theory }}=-4.83000$ & $\mu \mathrm{T}$ \\
\hline 16 & 0.011 & 0.0031 \\
32 & 0.043 & 0.0124 \\
64 & 0.173 & 0.0516 \\
128 & 0.690 & 0.244 \\
256 & 2.764 & 3.658 \\
\hline Case $2: \theta=45^{\circ} B_{d}(\mathbf{r})_{\text {theory }}=-4.95750$ & $\mu \mathrm{T}$ \\
\hline 16 & -0.0011 & -0.0013 \\
32 & -0.0040 & -0.0047 \\
64 & -0.0083 & -0.0096 \\
128 & 0.0502 & 0.0622 \\
256 & 0.7615 & 7.947 \\
\hline
\end{tabular}

matrix dimension. The cylinder was analyzed both parallel to the main magnetic field and at an angle of $45^{\circ}$. The results are summarized in Table 5.3. The deviation increases dramatically with radius but the results for $\Delta C$ remain reasonable up to a radius of 64 pixels; which is much larger than the vessel size that will be encountered in clinical situations. Figure 5.4 shows profiles of $B_{d}(\mathbf{r})$, for the various radii along the $y$-axis for one pixel for both $0^{\circ}$ and $45^{\circ}$. The trend in the $\%$ error for $B_{d}(\mathbf{r})$ can be clearly seen in Fig. 5.5; the error is proportional to radius squared, for the case where $\theta=0^{\circ}$, which is also proportional to the cross-sectional area of the cylinder. The $\theta=45^{\circ}$ case appears to have a higher order dependence on the radius. 

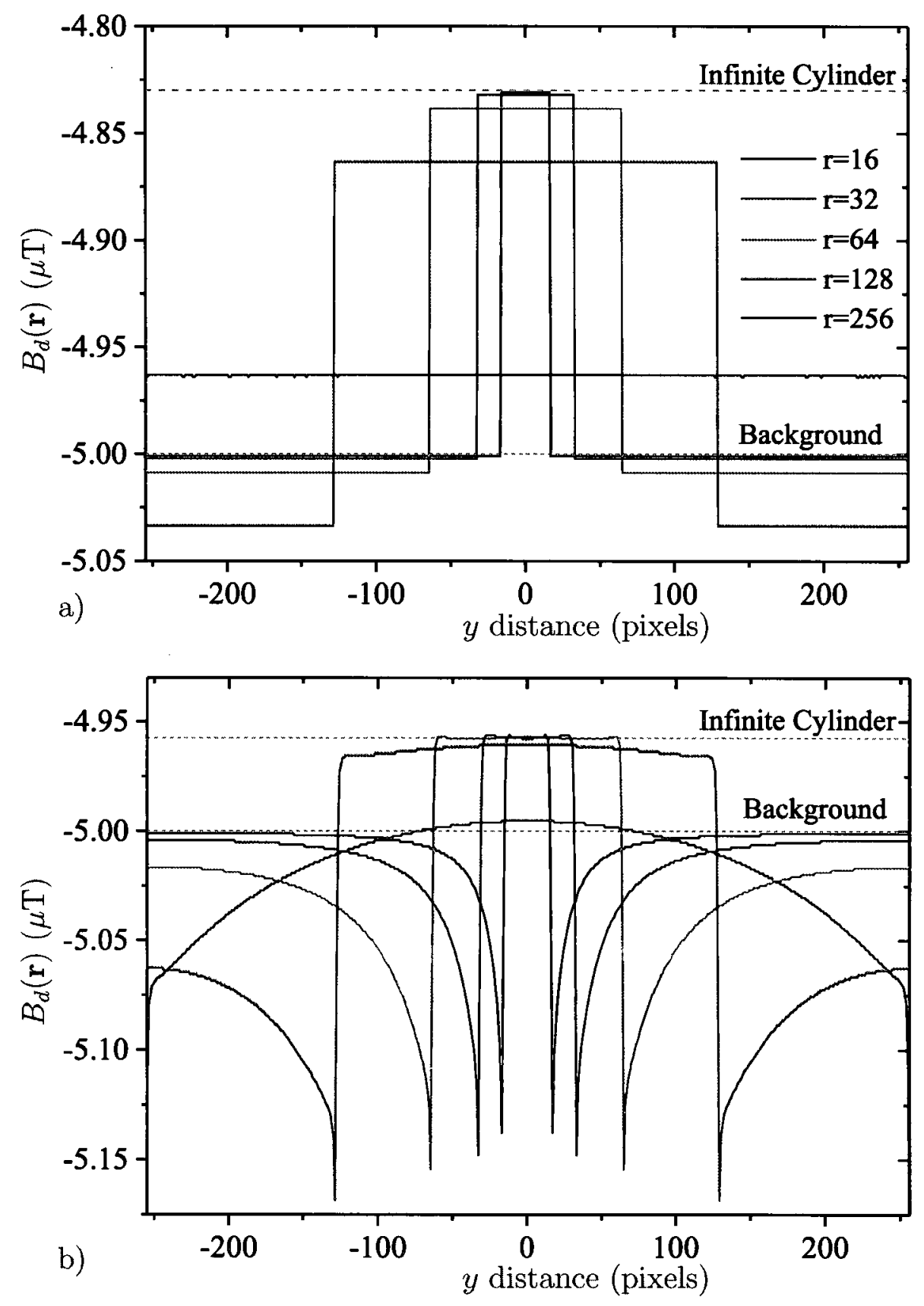

Figure 5.4: Profiles for an infinitely long cylinder of various radii and angles of a) $0^{\circ}$ and b) $45^{\circ}$ with respect to $B_{0} \hat{z}$. The cylinder was filled with a $1 \mathrm{mM}$ solution of CA and was surrounded by water. The theoretical $B_{d}(\mathbf{r})$ values for the inside an infinitely long cylinder and for the background are indicated in the graphs as dashed lines. 


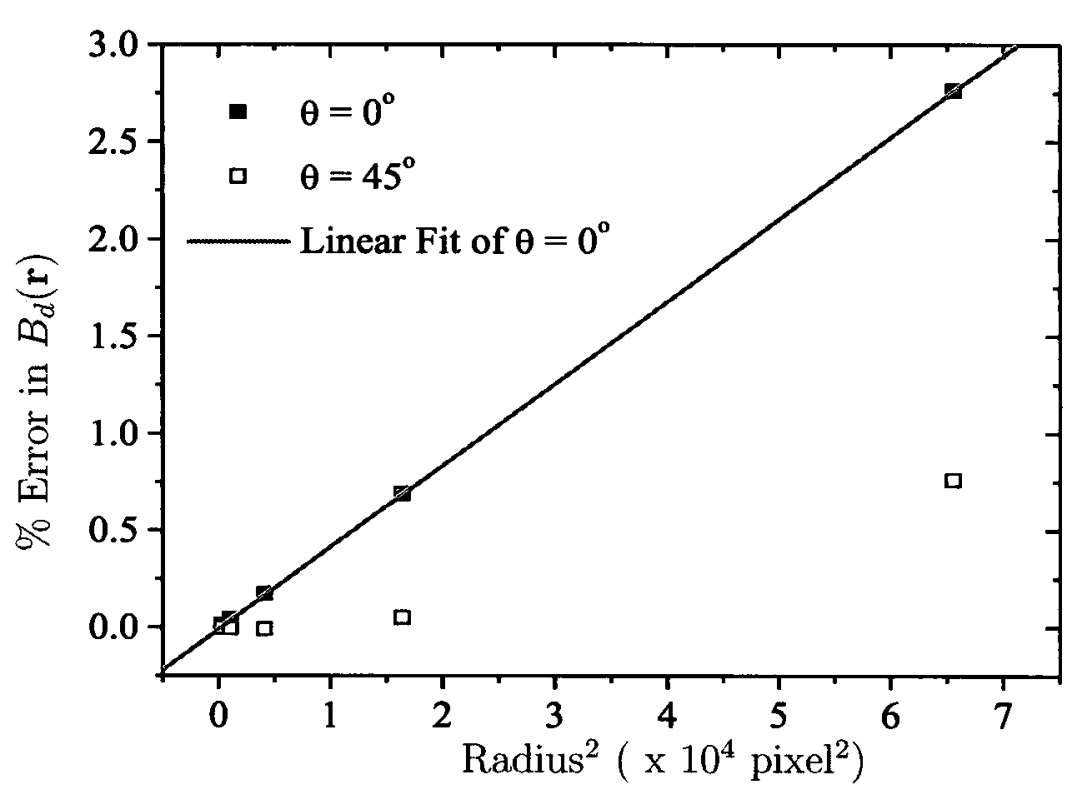

Figure 5.5: The percent error in $B_{d}(\mathbf{r})$ versus radius for a single cylinder at $\theta=0^{\circ}$ (closed squares) and $\theta=45^{\circ}$ (open squares) with respect to $B_{0} \hat{z}$, for a matrix size of $512^{3}$. The cylinder was filled with a $1 \mathrm{mM}$ solution of CA and was surrounded by water. The linear fit for $\theta=0^{\circ}$ (orange line) was $y=(4.2174 \pm 0.0011) \times 10^{-4} x-$ $(1 \pm 3) \times 10^{-5}, R=1, p<0.0001$.

\section{Total Volume}

Next the case of multiple tubes was explored to further investigate what happens when the volume of the tube is increased. To do this an additional tube was added to the $512^{3}$ volume which already contained 1 tube of radius 16 pixels. The radius of the second tube was varied from 0 (no tube) to 128 pixels as indicated in the first column of Table 5.4. Both tubes contained the same [CA], $C=1 \mathrm{mM}$, and were parallel with the external magnetic field. The distance between the cylinders and the location of the cylinders had no effect on the calculation of $B_{d}(\mathbf{r})$ (for this case of $\theta=0^{\circ}$ ).

Theoretically, since both cylinders are parallel to $B_{0} \hat{z}$, there should be no interaction between them at all. However, the results clearly show that the magnetic field inside the first cylinder is affected by the presence of the other cylinder. These results are presented in Table 5.4. The volume of the two cylinders were combined to determine the equivalent radius if the total volume was from one cylinder. This 
Table 5.4: Simulation results for $B_{d}(\mathbf{r})$ and $C$ for an infinitely long cylinder of radius 16 pixels parallel to $B_{0} \hat{z}$, with the addition of a second parallel cylinder of varying radius. The cylinders were both filled with $1 \mathrm{mM}$ solution of CA and was surrounded by water.

\begin{tabular}{|c|c|c|c|}
\hline Radius $_{t u b e 2}$ (pixels) & Radius $_{\text {equiv }}$ (pixels) & $\%$ Error for $B_{d}(\mathbf{r})$ & $\Delta C(\mathrm{mM})$ \\
\hline \multicolumn{4}{|c|}{$\theta=0^{\circ} B_{d}(\mathbf{r})_{\text {theory }}=-4.83000 \mu \mathrm{T}$} \\
\hline 0 & 16.0000 & 0.011 & 0.0031 \\
\hline 16 & 22.6274 & 0.021 & 0.0061 \\
\hline 32 & 35.7771 & 0.054 & 0.0155 \\
\hline 64 & 65.9697 & 0.183 & 0.0549 \\
\hline 128 & 128.996 & 0.701 & 0.249 \\
\hline
\end{tabular}

is represented as Radius equiv $_{\text {in }}$ in the table. Fig. 5.6 shows both the results from this section and the previous section as well as a linear fit to the data. The results for $\%$ error for $B_{d}(\mathbf{r})$ as a function of the radius of the single cylinder are in very good agreement with the $\%$ error for $B_{d}(\mathbf{r})$ results as a function of the equivalent radius for the two cylinders.

\section{Concentration Dependence}

Another potential source of variation in the error between the simulated and theoretical results is the difference between the susceptibility inside and outside the cylinder. To test this, the $[\mathrm{CA}]$ inside the vessel was varied from $0.1 \mathrm{mM}$ to $10 \mathrm{mM}$. As in the previous experiment, the cylinder had a radius of 16 pixels in a $512^{3}$ matrix. The simulation was done for a cylinder at $0^{\circ}$ with respect to $B_{0} \hat{z}$, where there should be no interaction between the inside and the outside as well as for $45^{\circ}$, where interaction is expected.

The results are presented in Table 5.5. Note that the last column is the error in $[\mathrm{CA}]$ per $\mathrm{mM}$ in order to facilitate comparison between the different [CA] levels. Table 5.5 shows that the error is generally proportional to $[\mathrm{CA}]$ although all the errors 


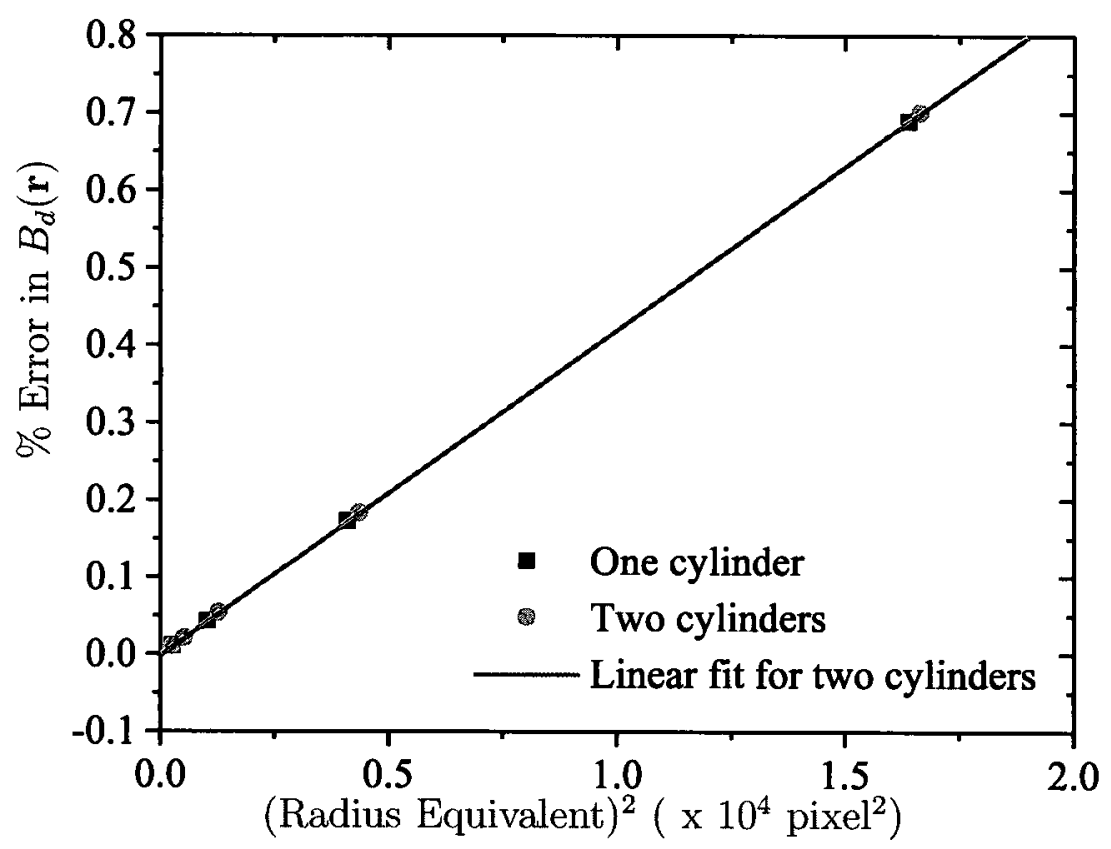

Figure 5.6: Percent error in $B_{d}(\mathbf{r})$ for the equivalent radius for a matrix size of $512^{3}$. The linear fit for $\theta=0^{\circ}$ (orange line) was $y=(4.213 \pm 0.003) \times 10^{-5} x-(2 \pm 2) \times 10^{-4}$, $R=1, p<0.0001$.

are small. Even for $10 \mathrm{mM}$, the error in $[\mathrm{CA}]$ is only $0.3 \%$ for $\theta=0^{\circ}$.

\section{Smoothing}

There are some cases in the clinical setting where the region growing software produces boundaries between the vessel and the surrounding tissue which is jagged and smoothing is applied to create a more continous and uniform vessel. This section explores the effect of smoothing on a straight tube of radius 16 pixels at $45^{\circ}$ with respect to $B_{0} \hat{z}$. This was applied as a nearest neighbour average (nna) with a kernel length of 3 pixels in each dimension and implemented by using the smooth function available in IDL. The results are presented in Table 5.6 and Fig. 5.7. As can be seen from Fig. 5.7, the discontinuity at the edge is reduced by the smoothing but the $B_{d}(\mathbf{r})$ value in the center of the tube remains the same. 
Table 5.5: Simulation results for $B_{d}(\mathbf{r})$ and $C$ for an infinitely long cylinder of radius 16 pixels at various $[\mathrm{CA}] \mathrm{s}$.

\begin{tabular}{|cccc|}
\hline $\mathrm{C}(\mathrm{mM})$ & $B_{d}(\mathbf{r})_{\text {theory }}(\mu \mathrm{T})$ & \% Error for $B_{d}(\mathbf{r})$ & $\Delta C / C$ \\
\hline \multicolumn{5}{|c|}{$\theta=0^{\circ}$} \\
\hline 0.1 & -4.98300 & 0.001 & -0.0031 \\
0.5 & -4.91500 & 0.0052 & -0.0030 \\
1 & -4.83000 & 0.011 & -0.0031 \\
5 & -4.15000 & 0.0623 & -0.00305 \\
10 & -3.30000 & 0.157 & -0.00305 \\
\hline \multicolumn{5}{c}{$\theta=45^{\circ}$} \\
\hline 0.1 & -4.99580 & -0.001 & -0.01 \\
0.5 & -4.97880 & -0.002 & -0.004 \\
1 & -4.95750 & -0.001 & -0.001 \\
5 & -4.78750 & -0.059 & -0.0012 \\
10 & -4.57500 & -0.012 & -0.0012 \\
\hline
\end{tabular}

Table 5.6: Simulation results for $B_{d}(\mathbf{r})$ and $C$ for an infinitely long cylinder of radius 16 pixels with smoothing. The cylinder was filled with a $1 \mathrm{mM}$ solution of CA and was surrounded by water. The term "nna $3 \times 2$ " indicates that the smoothing was applied twice.

\begin{tabular}{|c|c|c|}
\hline Smoothing & $\%$ Error for $B_{d}(\mathbf{r})$ & $\Delta C(\mathrm{mM})$ \\
\hline \multicolumn{3}{|c|}{$\theta=45^{\circ} B_{d}(\mathbf{r})_{\text {theory }}=-4.95750 \mu \mathrm{T}$} \\
\hline none & -0.001 & -0.001 \\
\hline nna 3 & -0.0006 & -0.0007 \\
\hline nna $3 \times 2$ & -0.0005 & -0.0005 \\
\hline
\end{tabular}




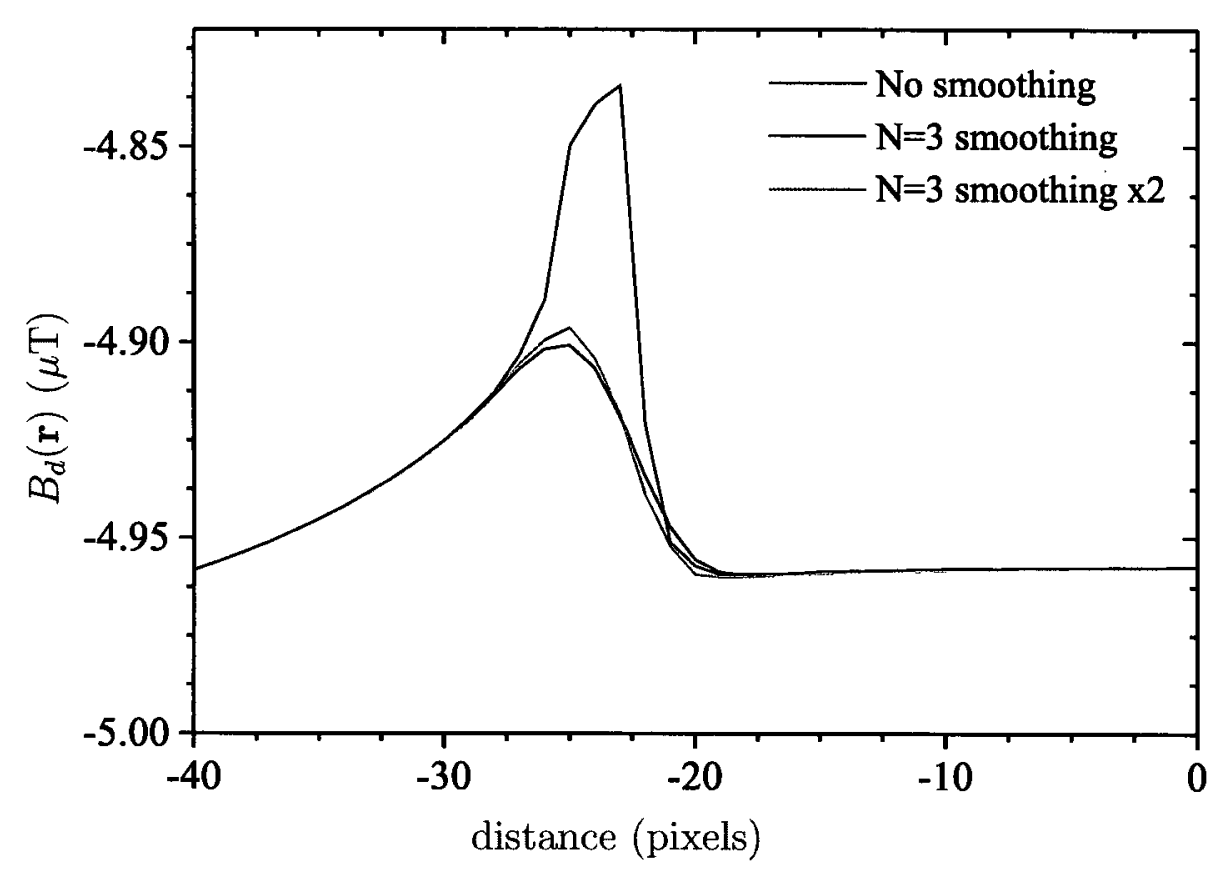

Figure 5.7: Simulated $B_{d}(\mathbf{r})$ profile showing the effects of smoothing.

\subsubsection{Phantom Validation}

In the previous section, the validation of the dipole approximation method on a simulated vessel of simple geometry was addressed. However, the goal of this chapter is to be able to accurately relate the phase change seen in a real MRI image to the [CA]. With this in mind, the next logical step is to apply this dipole approximation method to a phantom with a known [CA] and see how well the phase accumulation values determined from the simulations agree with those obtained directly from experiment.

Results for straight and curved phantoms were presented in Chapter 4 in the context of validating the phase measurement. The results for a complex geometry phantom are presented in this section. Here, the goal is to assess the validity of the computational method and not the experimental method; thus, only $1 \mathrm{mM}$ and 10 $\mathrm{mM}$ solutions of CA were used. As described in Section 5.2.3, the complex phantom should have the same $[\mathrm{CA}]$ everywhere and it does not change with time. Thus, the $\phi$ accumulation measured in the phantom can be compared to that calculated using 
the dipole approximation method.

To generate the susceptibility map required by the simulation algorithm, a magnitude image was also generated from the image data. The interior of the tubing was identified on this image by using a thresholding algorithm and these pixels were assigned values equal to the susceptibility for the known [CA]. The simulation algorithm was then run with this susceptibility map to determine the changes in the magnetic field that the CA would be expected to cause. The associated phase accumulation was then calculated. This process was repeated for $C=1 \mathrm{mM}$ and $C=10 \mathrm{mM}$ and the difference in the predicted phase accumulation, $\Delta \phi_{s i m}$, was compared with the experimentally determined phase accumulation, $\Delta \phi_{\text {exp }}$, on a pixel-by-pixel basis.

The histogram presented in Fig. 5.8 shows the ratio of $\Delta \phi_{\text {exp }}$ to $\Delta \phi_{s i m}$. The mean and mode are very close to the ideal value of unity. The histogram appears to be symmetric without any tendency toward over or underestimation. There is some spreading of the distribution as indicated by the standard deviation of $0.172 \mathrm{rad}$, which corresponds to a percent error in $[\mathrm{CA}]$ of $17.2 \%$ relative to the measured value.

The data was processed in several ways in order to determine the cause of the spreading and to attempt to correct it. The statistics for the results under several data acceptance criteria are presented in Table 5.7. First, removing data with low $\mathrm{SNR}_{\phi}$ was considered. The random noise present in the phase data is independent of the amount of phase accumulation. At certain orientations however, the phase accumulation is itself very low, resulting in a lower $\mathrm{SNR}_{\phi}$; this is illustrated in Fig. 5.9. The predicted and measured phase accumulations match fairly well. However, both values are close to zero in the center of the tube and dividing one by the other can lead to an unrealistic result, as shown in the bottom panel of Fig. 5.9. Unrealistic values are eliminated from the dataset by applying windowing. One method to deal with this is to retain only the pixels where the amount of simulated phase accumulation falls withing a certain percentage of the phase accumulation predicted for a straight 


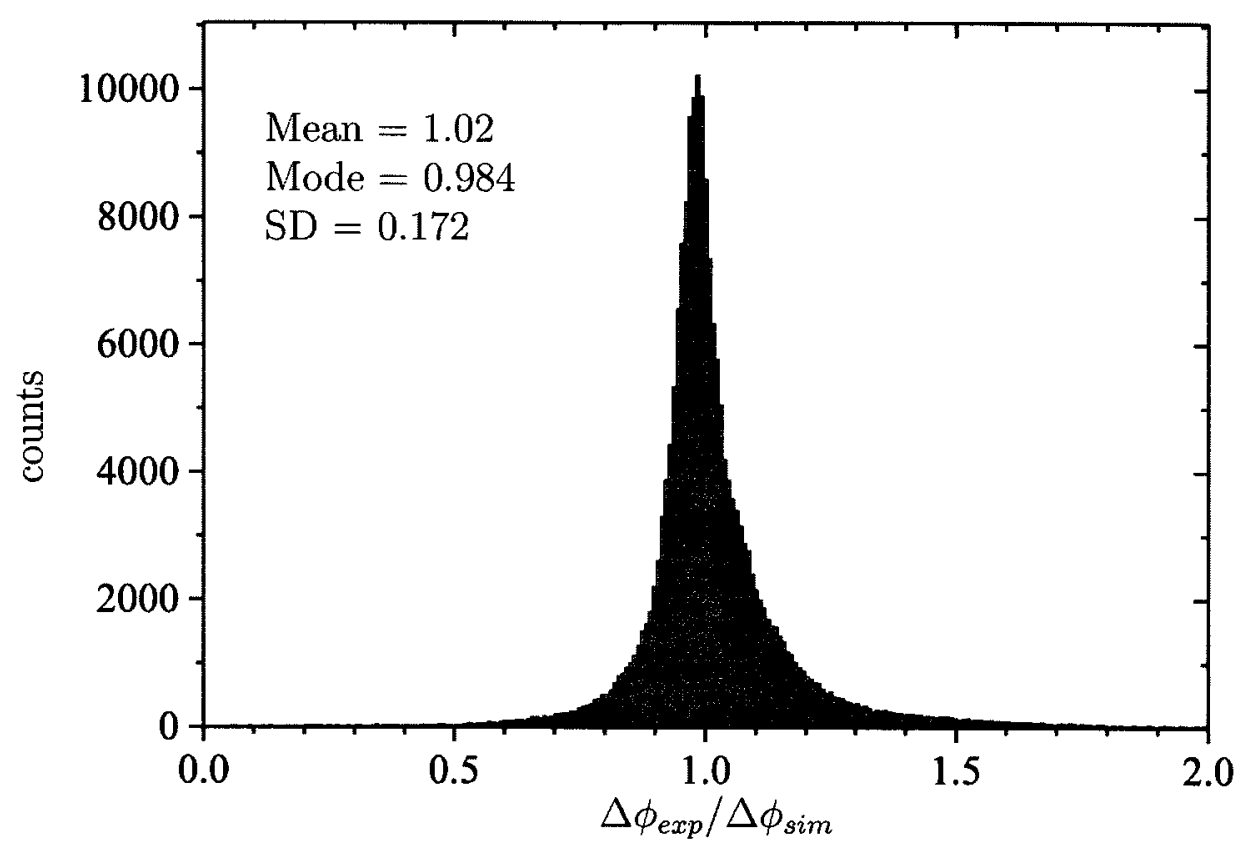

Figure 5.8: Histogram showing the ratio of the phase accumulation measured in experiments to that predicted by simulation. Total counts $\approx 200000$.

vessel parallel to $B_{0} \hat{z}$, since this is the maximum phase accumulation. The first three lines of Table 5.7 show the results when all data $(0 \%)$ or data greater than $10 \%$ and $25 \%$ of the straight tube value are retained.

Another potential source of error is the difficulty in achieving a correct simulated value near the edges, owing to the discrete Fourier transform. The last two lines of Table 5.7 show the effect of removing some of the pixels originating at the edges of the phantom. This was done with a thresholding algorithm using a mask created from the original magnitude data with a higher threshold than the one used to generate the simulated magnetic susceptibility map, since the edges of the magnitude data tend to have lower values because of partial volume effects. This amounts to removing approximately a one pixel ring around the edge of the tube. 

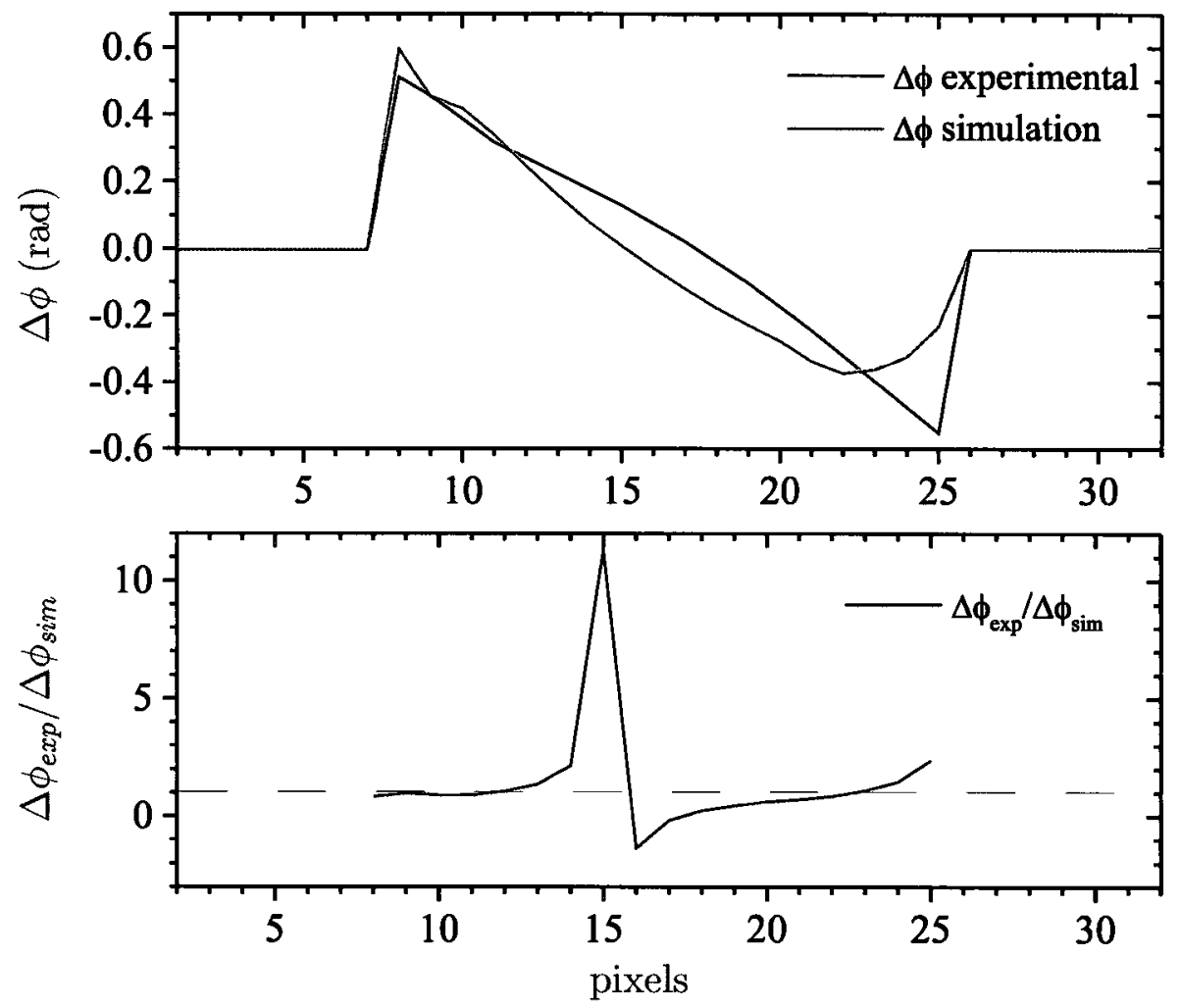

Figure 5.9: Profile of the phase accumulation measured in experiments to that predicted by simulation through a portion of tube where low phase accumulation contributes to high errors in $\Delta_{e x p} / \Delta_{s i m}$.

Table 5.7: Statistics for $\Delta \phi_{\exp } / \Delta \phi_{\text {sim }}$ under different inclusion criteria.

\begin{tabular}{|cccc|}
\hline Set Up & mean & mode & SD \\
\hline mask, $>0 \%$ & 1.02 & 0.984 & 0.172 \\
mask, $>10 \%$ & 1.02 & 0.984 & 0.155 \\
mask, $>25 \%$ & 1.01 & 0.984 & 0.132 \\
mask increase, $>0 \%$ & 1.01 & 0.984 & 0.153 \\
mask increase, $>10 \%$ & 1.00 & 0.984 & 0.133 \\
\hline
\end{tabular}




\subsubsection{Straight Tube Approximation}

Simulations to assess the validity of approximating a curved vessel as an isolated infinite straight cylinder at an angle parallel to the tangent to the curve at the point of interest are presented in this section. First, the effect of nearby vessels is studied to validate the assumption that the tube must be isolated from other tubes. Next the assumption of an infinite cylinder is challenged by reducing the length of the tube. Finally, and perhaps most importantly, the effect of curvature will be assessed by using two different shapes. The first shape, a torus, is well-defined and symmetric. This shape is used to assess the effect of varying the radius of curvature. The second structure considered was generated from a second order polynomial fit applied to the SSS (see Section 6.2 for details). This was done to simulate a curved vessel and the results were compared to those obtained by taking the tangent to the curve at select points.

The radius of the cylinder was 16 pixels, the $[\mathrm{CA}]$ was $1 \mathrm{mM}$ and the matrix size was $512^{3}$, unless otherwise stated. The angle between the tangent to the curve at the point of interest and $B_{0} \hat{z}$ was calculated analytically.

\section{Nearby Objects}

Vessels which are not parallel to the magnetic field will cause a magnetic field shift in other nearby objects. The resulting magnetic field in any given position is the superposition of the contribution from all objects. To give a sense of this contribution Fig. 5.10 shows the $B_{d}(\mathbf{r})$ profile along the $z$-axis for two infinite cylinders that are parallel to each other and perpendicular to the external magnetic field. The theoretical superposition is also presented. As expected, the field inside each cylinder is significantly affected by the presences of nearby cylinders. 


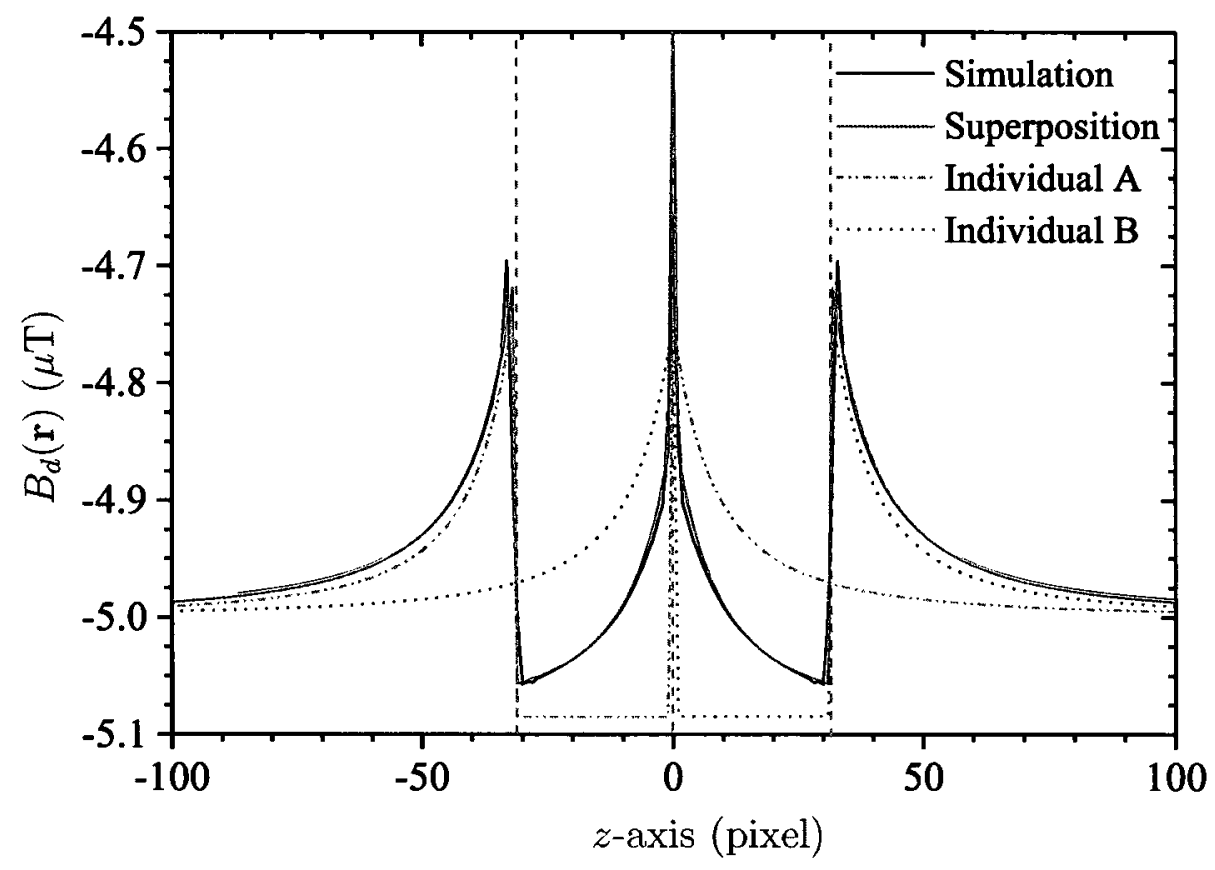

Figure 5.10: Profiles for two infinite cylinders at $90^{\circ}$ to $B_{0} \hat{z}$, each with a 16 pixel radius and $1 \mathrm{mM}$ of $\mathrm{CA}$.

\section{Infinite Length Approximation}

The effect of varying the length of the cylinder from 32 pixels to 512 pixels is presented in Table 5.8. Figure 5.11 shows the $B_{d}(\mathbf{r})$ profiles along the $z$-axis for cylinders of lengths 32 and 256 pixels. The theoretical profile for a sphere with diameter 32 pixels and the value for an infinitely long cylinder are also plotted. From this it can be seen that the simulation results are more accurate when the length of the cylinder is long compared to the radius, thus giving a better approximation to an infinitely long cylinder. When the length becomes equal to the diameter of the cylinder the magnetic field shift is more similar to that of a sphere than that of an infinite cylinder, which is not surprising.

\section{Torus}

To test the effect of the curvature of the vessel on the magnetic field shift inside that vessel, tori were simulated with radii of curvature from 64 to 256 pixels. An example 
Table 5.8: Simulation results for $B_{d}(\mathbf{r})$ and $C$ for cylinders of radius 16 pixels and various lengths.

\begin{tabular}{|c|c|c|}
\hline Length (pixels) & $\%$ Error for $B_{d}(\mathbf{r})$ & $\Delta C(\mathrm{mM})$ \\
\hline \multicolumn{3}{|c|}{$\theta=0^{\circ} B_{d}(\mathbf{r})_{\text {theory }}=-4.83000 \mu \mathrm{T}$} \\
\hline 32 & 3.2055 & 1.0203 \\
\hline 64 & 1.138 & 0.4780 \\
\hline 128 & 0.3174 & 0.09910 \\
\hline 256 & 0.0805 & 0.0234 \\
\hline 512 & 0.011 & 0.0031 \\
\hline
\end{tabular}

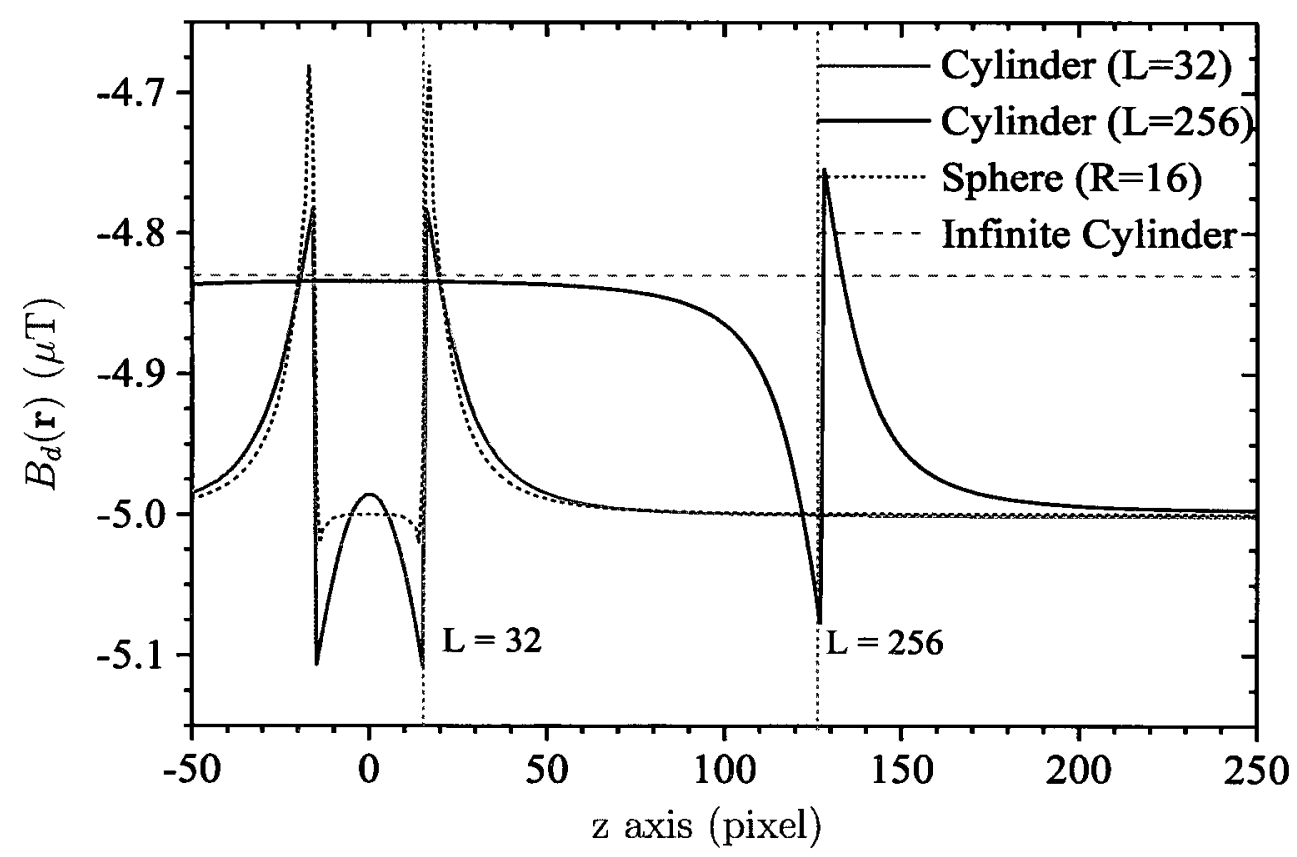

Figure 5.11: Profile along the $z$-axis for $B_{d}(\mathbf{r})$ for a cylinder 32 pixels long, a cylinder 256 pixels long, as well as the theoretical value for a sphere of diameter 32 pixels and a cylinder of infinite length. 
$B_{d}(\mathbf{r})$ map for a radius of curvature of 128 pixels is given in Fig. 5.12. The results are also presented in Table 5.9. The first column indicates the angle of the tangent of the torus with respect to the main magnetic field. The second is the value of $B_{d}(\mathbf{r})$ at that angle for an infinite straight tube. The third column indicates the percent error for the straight tube approximation value relative to the $B_{d}(\mathbf{r})$ value from this simulation, assuming that the simulation provides the "theoretical" value. The result is also presented in terms of $\Delta C$ in the next column. This is the error which would result if the straight vessel approximation was used to calculate the geometry factor (see Eq. 5.3) instead of using a simulated torus and the dipole approximation method.

The values quoted in Table 5.9 are for the center of the torus with a radius of curvature of 128 pixels, as is consistent with the previous analysis. It is important to note that the magnetic field shift measured inside the torus is not uniform (see Fig. 5.13). The extent of the variation depends on the radius of curvature of the tube. For this reason, an additional column giving the range of [CA]s is given in Table 5.9. This range is calculated by taking the difference between the maximum and minimum [CA] found along a line of length equal to half the diameter of the tube which intersects the central pixel and is normal to the tangent at that point in the curve. It can be seen that the value in the center of the torus corresponds well to that predicted by the straight tube approximation and the $[\mathrm{CA}]$ obtained for the center of the tube are in good agreement with the assumed value of $1 \mathrm{mM}$.

Fig. 5.14 shows the error in [CA] for three different radii of curvature for tangential angles between $0^{\circ}$ and $30^{\circ}$. As expected, a torus with a larger radius of curvature is a better approximation to the straight vessel than a more curved torus.

\section{Polynomial}

An additional test of the straight tube approximation was done using a shape similar

to the superior sagittal sinus. A tube with $2^{\text {nd }}$ order curvature was generated as 


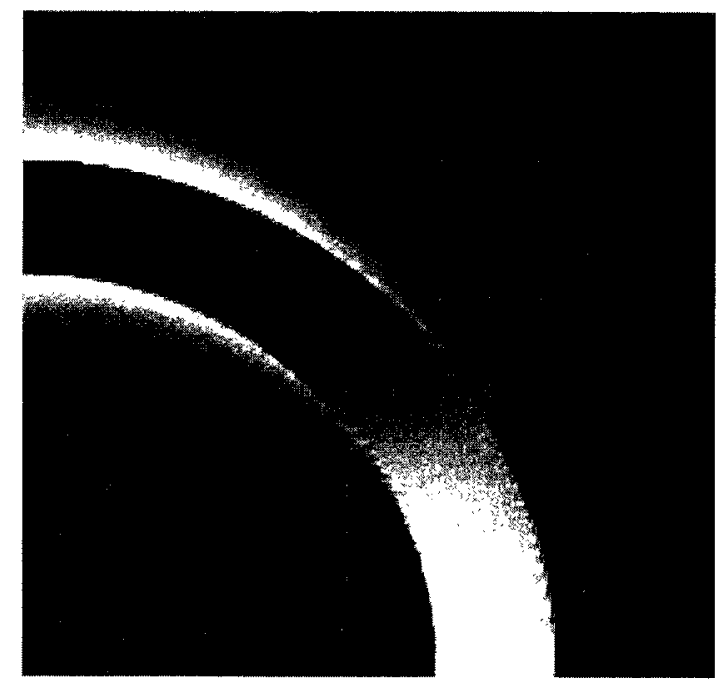

Figure 5.12: One quadrant of the $B_{d}(\mathbf{r})$ map for a torus with a radius of curvature of 128 pixels and a radius of 16 pixels containing a $1 \mathrm{mM}$ solution of $\mathrm{CA}$.

Table 5.9: Simulation results for $B_{d}(\mathbf{r})$ and $C$ for a torus with radius of curvature equal to 128 pixels and a radius of 16 pixels. The $B_{d}(\mathbf{r})$ values were compared to $B_{d}(\mathbf{r})_{s t}$, the field expected for an infinitely long straight cylinder oriented parallel to the tangent to the torus at the given location.

\begin{tabular}{|ccccc|}
\hline Tangent $\left(^{\circ}\right)$ & $B_{d}(\mathbf{r})_{s t}(\mu \mathrm{T})$ & $\%$ Error for $B_{d}(\mathbf{r})$ & $\Delta C(\mathrm{mM})$ & $C$ range $(\mathrm{mM})$ \\
\hline 0 & -4.83000 & -0.121 & -0.0343 & 0.1633 \\
2.5 & -4.83049 & -0.132 & -0.0377 & 0.1624 \\
5 & -4.83194 & -0.146 & -0.0419 & 0.1660 \\
10 & -4.83769 & -0.118 & -0.0351 & 0.1677 \\
15 & -4.84708 & -0.117 & -0.0372 & 0.1639 \\
20 & -4.85983 & -0.0985 & -0.0341 & 0.1659 \\
25 & -4.88089 & -0.0720 & -0.0282 & 0.1539 \\
30 & -4.89824 & -0.00510 & -0.0235 & 0.1570 \\
35 & -4.91389 & -0.011 & -0.0062 & 0.1494 \\
40 & -4.93536 & 0.0051 & 0.0039 & 0.1372 \\
45 & -4.95750 & 0.017 & 0.020 & 0.0855 \\
\hline
\end{tabular}




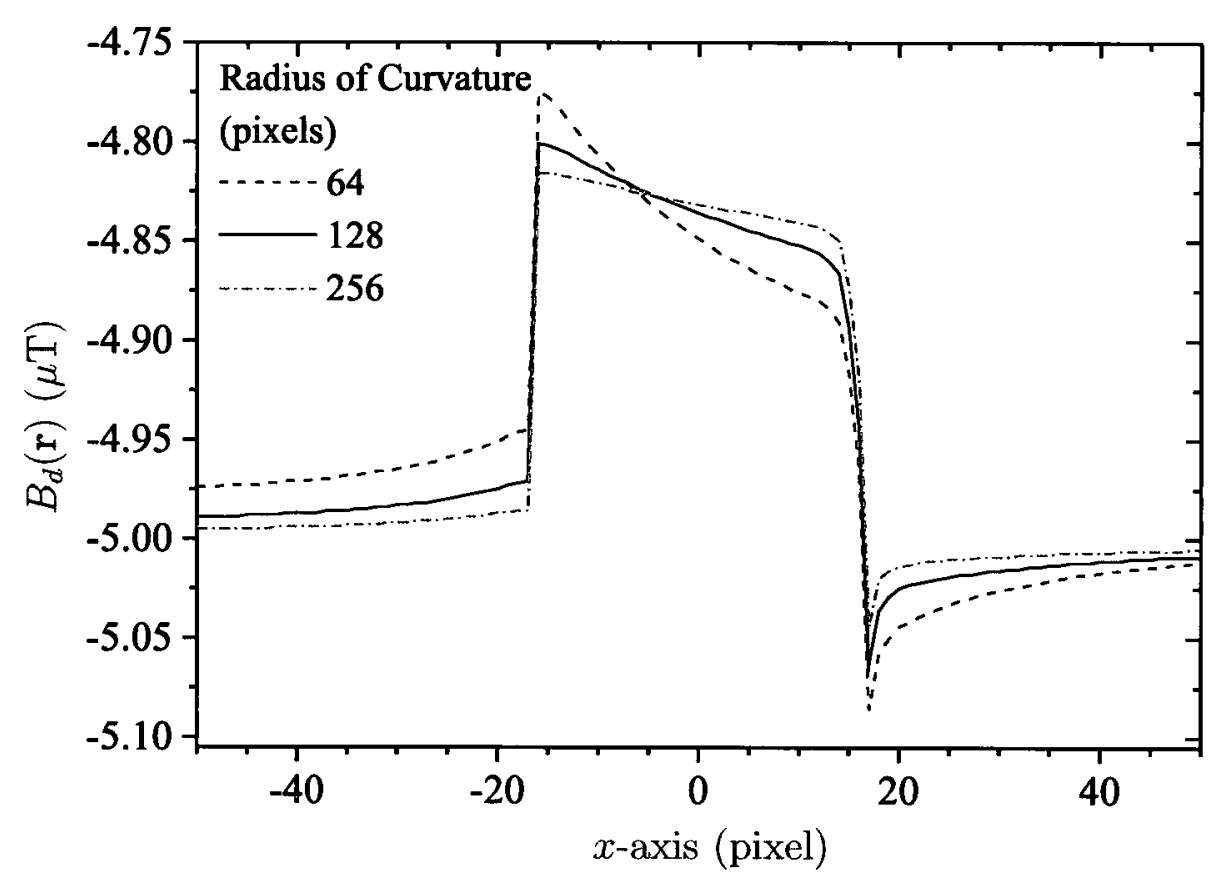

Figure 5.13: Typical profiles for tori with various radii of curvature, each with a 16 pixel radius. For these profiles the tangent formed an angle of $0^{\circ}$ with $B_{0} \hat{z}$.

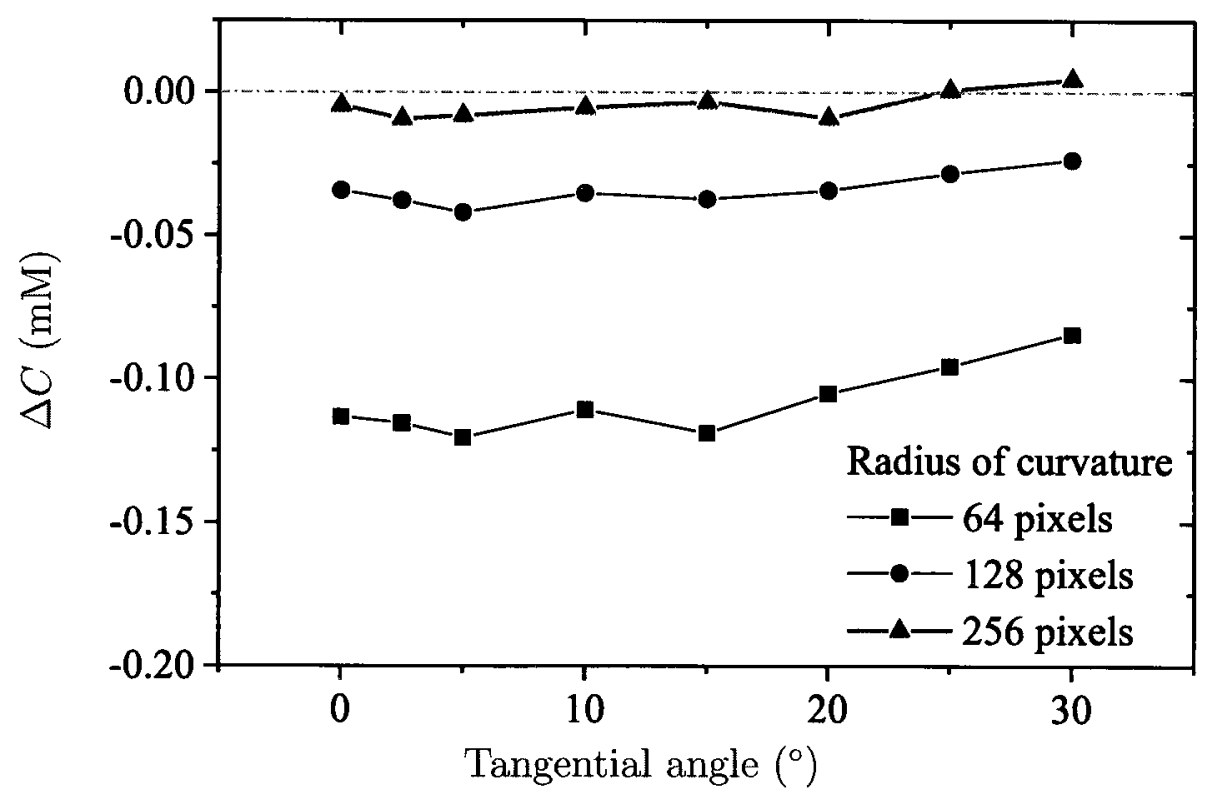

Figure 5.14: The error in $[\mathrm{CA}]$ versus angle for several radii of curvature of the torus. 
described in Section 5.2.2. The results are presented in Table 5.10. The first column of the table indicates the angle, with respect to $B_{0} \hat{z}$, of the tangent to the polynomial at the location of the four dynamic slices taken as part of the brain tumour protocol (see Section 6.2). The second column contains the values of $B_{d}(\mathbf{r})$ at that angle for an infinite straight tube. The third column indicates the percent error for the straight tube approximation value relative to the $B_{d}(\mathbf{r})$ value from this simulation, assuming that this simulation provides the "theoretical" value. The corresponding values of $\Delta C$ are presented in the next column. Again, this is the error which would result if the straight vessel approximation was used to calculate the geometry factor instead of using a simulated polynomial fit vessel and the dipole approximation method. Finally, as in the previous section, a [CA] range across the tube is given. This range was calculated slightly differently, in order to make it more similar to that which would be measured experimentally. In this case, the range is for the maximum and minimum found for a circle of radius equal to half that of the tubes. It was placed in the same $x y$-plane (transverse).

\subsubsection{Method Applied to Clinical Data}

As explained in Section 5.2.4, a region growing algorithm, applied to the 3D image data sets from the brain tumour study presented in Chapter 6, was used to segment out the SSS. A susceptibility value, corresponding to $C=1 \mathrm{mM}$, was then applied to the interior of the SSS. The material outside the SSS was assumed to be water. This was used as the susceptibility map for the simulation. The results are presented in Table 5.11 for a 4-pixel ROI, placed in the same locations as in the final section of Section 5.3.3. The first column is the angle of the vessel with respect to $B_{0} \hat{z}$, at the location of the four dynamic slices from the brain tumour protocol. This angle was determined using the polynomial fits defined in Table 5.1. The second column is the geometry correction factor, $g(\mathbf{r})_{s t}$, for an infinitely long cylinder at that angle 
Table 5.10: Comparison between $B_{d}(\mathbf{r})$ calculated using a simulated polynomial and using the straight tube approximation.

\begin{tabular}{|ccccc|}
\hline Tangent $\left({ }^{\circ}\right)$ & $B_{d}(\mathbf{r})_{s t}(\mu \mathrm{T})$ & \% Error for $B_{d}(\mathbf{r})$ & $\Delta C(\mathrm{mM})$ & $C$ range $(\mathrm{mM})$ \\
\hline \multicolumn{5}{c|}{ Patient P006-S001 } \\
\hline 24.4 & -4.87367 & 0.013 & 0.0051 & 0.0259 \\
16.7 & -4.85113 & -0.0091 & -0.0030 & 0.0524 \\
8.34 & -4.83536 & -0.0319 & -0.00938 & 0.07173 \\
-0.425 & -4.83001 & -0.0401 & -0.0114 & 0.07256 \\
\hline \multicolumn{5}{c}{ Patient P012-S001 } \\
\hline 5.69 & -4.83250 & -0.133 & -0.0384 & 0.0389 \\
9.97 & -4.83765 & -0.0391 & -0.0117 & 0.0336 \\
14.2 & -4.84524 & -0.0153 & -0.00480 & 0.0284 \\
18.2 & -4.85482 & -0.00576 & -0.001926 & 0.0237 \\
\hline \multicolumn{5}{c}{ Patient P017-S001 } \\
\hline 6.96 & -4.83374 & -0.0208 & -0.00605 & 0.0287 \\
3.38 & -4.83089 & -0.0196 & -0.0560 & 0.0287 \\
-0.228 & -4.83000 & -0.0213 & -0.00604 & 0.0302 \\
-3.83 & -4.83114 & -0.0249 & -0.00712 & 0.0299 \\
\hline
\end{tabular}


with respect to the main magnetic field. The third column is the geometry correction factor, $g(\mathbf{r})_{c l}$, as determined by using the clinical data to generate a susceptibility map and applying the dipole approximation method. Finally the percent difference between the two methods is presented in the final column. Note that an additional patient example (P008-S001) is added as compared to Section 5.3.3. This is because the vessel region of interest in this patient was relatively straight and thus was not an interesting case for the validation of the straight tube approximation but illustrates the validity of the simulation method and thus is presented here.

\subsection{Discussion}

\subsubsection{Assess Method on Known Geometry}

The results presented in Section 5.3.1 show that simulations using the dipole approximation method to estimate the magnetic field shift caused by the presence of a CA are valid and that this technique is an appropriate way of estimating the resulting phase change in the blood vessels. The error in the estimated [CA] is well below the experimental error observed in Chapter 4 for all but a few cases.

In particular, the results show that the accuracy of the simulations is high for all tube orientations. For a 16 pixel radius cylinder filled with a $1 \mathrm{mM}$ solution of $\mathrm{CA}$, all the predicted magnetic fields and $[\mathrm{CA}] \mathrm{s}$ were accurate to $\leq 0.011 \%$ and $\leq 0.0064 \mathrm{mM}$, respectively. The results also showed that the accuracy of the simulation decreases with the proportion of the matrix occupied by the cylinder. However, for a radius of 32 pixels, which is larger than the radius of vessels that would normally be found in clinical images, the method predicts $[\mathrm{CA}]$ to within about $1.2 \%$. The simulations showed that the accuracy of the simulated results decreased with increasing concentration with $\Delta C / C$ remaining constant. But even for the worst case, the simulations 
Table 5.11: A comparison of the geometry factor calculated using the straight vessel approximation, $g(\mathbf{r})_{s t}$, and a segmented vessel from the SSS, $g(\mathbf{r})_{c l}$.

\begin{tabular}{|cccc|}
\hline Tangent $\left(^{\circ}\right)$ & $g(\mathbf{r})_{s t}$ & $g(\mathbf{r})_{c l}$ & \% Difference \\
\hline \multicolumn{5}{|c|}{ Patient P006-S001 } \\
\hline 24.4 & 1.34570 & 1.29677 & 3.70 \\
16.7 & 1.14194 & 0.99929 & 13.3 \\
8.34 & 1.03258 & 0.92561 & 10.9 \\
-0.425 & 1.00008 & 0.89913 & 10.6 \\
\hline \multicolumn{5}{|c|}{ Patient P012-S001 } \\
\hline 5.69 & 1.01750 & 1.13394 & -10.8 \\
9.97 & 1.05332 & 1.16896 & -10.4 \\
14.2 & 1.10870 & 1.16962 & -5.34 \\
18.2 & 1.18455 & 1.08256 & 9.00 \\
\hline \multicolumn{5}{c}{ Patient P017-S001 } \\
\hline 6.96 & 1.02250 & 1.03046 & -0.775 \\
3.38 & 1.00523 & 1.01789 & -1.25 \\
-0.228 & 1.00002 & 0.98159 & 1.86 \\
-3.83 & 1.00675 & 1.06400 & -5.53 \\
\hline \multicolumn{5}{c}{ Patient P008-S001 } \\
\hline 25.0 & 1.36636 & 1.41820 & -3.72 \\
25.3 & 1.37630 & 1.44141 & -4.62 \\
25.5 & 1.38643 & 1.36404 & 1.63 \\
25.8 & 1.39673 & 1.35372 & 3.13 \\
\hline
\end{tabular}


still performed very well, predicting the correct concentration to within about $0.3 \%$. The simulation results presented here show that, for infinitely long cylinders filled with a CA of known susceptibility, the methods predicts the [CA] to better than $1 \%$ for any cylinder orientation and for all clinically relevant concentrations and vessel radii.

The largest errors were observed at the boundaries of the objects. In Fig. 5.3, it can be observed that the profiles are rounded at the vessel/background boundary and they deviate from theory. This is because, with a discrete Fourier transform, the boundary (a step function) is being represented by a finite series. That is to say, $\mathrm{k}$-space is truncated and the high spatial frequencies needed to reproduce the sharp change at the boundary are missing. This discrepancy at the boundary is not a detrimental result in the application of this technique to DCE-MRI. As discussed in Chapter 4 , it is preferable to restrict the ROI drawn in the vessel to the inner pixels to avoid leakage and partial volumes at the boundary. Thus, in the simulation, the boundary information is not needed.

This effect can be reduced if desired, by increasing the k-space sampling range (i. e. use a larger matrix for the same field of view). For a sufficiently large vessel (in pixels, not $\mathrm{mm}$ ) the boundary can be avoided and the results in the central portion will be accurate, as shown in Fig. 5.3. On that note, images of small vessels, should be resampled so that measured pixels are sufficiently far away from the boundary to provide accurate values.

An additional consideration when optimizing the simulation is to consider the proportion of the matrix occupied by background material. The assumption that Eq. 5.7 is valid amounts to saying that all of the space outside the cylinder is filled with background material of susceptibility $\chi_{e}$ [25]. This assumption is clearly not rigorously true for the simulations presented here since the susceptibility is only defined for the finite subset of space corresponding to the $512^{3}$ susceptibility map provided to the 
simulation as input. Figure 5.5 shows that the error in the estimated magnetic field shift increases with the square of the radius of the cylinder and is different at different angles. In Fig. 5.6 it can seen that it is the total volume of the vessel in the matrix for a given orientation and not the radius of the measured vessel, which is important, since the cylinder measured in this case did not change radius. The results in Table 5.3 indicate that the error is, however, relatively small. When the radius is 64 pixels, the error in $[\mathrm{CA}]$ is within $5 \%$. To reduce this effect, a matrix with a large proportion of the volume filled with background is preferrable.

The following considerations should be made when constructing the susceptibility map for the application to the vessels in DCE-MRI. If the vessels are small, they should be resampled to mitigate against inaccuracies at the boundaries. Addition-

ally, the background must be sufficiently large that it satisfies the central $k$-space assumption discussed in the preceding paragraph. This means that the largest matrix size practically possible should be used. The size of the matrix is limited by available memory and computational time. The calculation of a magnetic field susceptibility map must be done on a per-patient basis. Fortunately, the hardware and software associated with an MRI scanner is specifically designed to efficiently perform Fourier transforms of relatively large datasets. The simulations presented here were performed in a reasonable amount of time (15 minutes) on a standard, consumer-level computer and thus would not be prohibitively time consuming for an MRI system. With these considerations in mind the dipole approximation can be successfully applied to DCE-MRI.

\subsubsection{Phantom Validation}

The results presented in Section 5.3.2 show that the dipole approximation method can be used to accurately predict the phase induced by the CA for a complex vessel geometry from measured data. This also shows that ROI selection for determining 
the arterial input function (AIF) or the venous output function (VOF) need not be restricted to a linear portion of the vessel or a specific orientation; the entire vessel can be used to calculate the AIF/VOF. This dramatically increases the SNR of the AIF/VOF. Alternatively, geometry-corrected, phase-derived AIFs/VOFs obtained throughout the imaging volume could be compared to assess the delay and dispersion of the bolus as it passes through the body.

It can be seen in Fig. 5.8 that there is some spreading of the histogram, indicating that the simulation is not correctly estimating $\Delta \phi$ in all locations. This could be owing to the difficulty in calculating the correct phase difference at the boundaries between the vessel and the background material. This effect was reduced by increasing the masking threshold and thus reducing the number of boundary pixels included. An alternative to increasing the masking threshold would be to extend the thickness of the simulated phantom.

Another instance where it is difficult to estimate $\Delta \phi$ is when there is little or no phase accumulation; see Fig. 5.9. This is not a problem in the simulation but it demonstrates that at some angles the phase difference is more susceptible to variations due to noise than others. It is always best to select a vessel parallel to the main magnetic field to achieve the highest $\mathrm{SNR}_{\phi}$, whenever possible. Applying a cutoff to remove pixels with low $\mathrm{SNR}_{\phi}$ improved the results, as can be seen in Table 5.7. An alternative approach would be to use a weighted average based on the simulated results when combining pixels to determine the AIF/VOF.

\subsubsection{Straight Tube Approximation}

The straight tube approximation provides a method for calculating the geometry factor without simulating the magnetic field shift for each patient. As stated in the previous chapter, it was hypothesized that for the case of mildly curved vessels the geometry factor can be approximated as that of an infinite straight tube at the angle 
equal to that formed by the tangent of the curved tube with respect to the main magnetic field. This hypothesis was assessed for the cases of a torus and a $2^{\text {nd }}$ order polynomial tube and was found to be successful in both cases. The concentration values predicted by the simulation are off by less than $0.04 \mathrm{mM}$ and $0.12 \mathrm{mM}$ for the torus with a radius of curvature to radius of 8 and the polynomial, respectively. These simulations, however, did not address the situation of branching of the vessels and thus, these areas should be avoided when selecting vessel ROIs. Future study could address this issue.

Another observation warranting future investigation is that a larger phase enhancement can sometimes be found outside the vessel, where no CA is present, than inside the vessel (see Fig. 5.12). This larger phase enhancement could potentially be exploited to increase the $\mathrm{SNR}_{\phi}$. This observation also highlights the fact that one cannot simply take the pixels with the highest phase accumulation as the intravascular pixels; one should use the magnitude image to locate the interior of the vessel.

\subsubsection{Clinical Cases}

The results from Section 5.3.4 show that the dipole approximation can be used to estimate the geometry factor directly from the clinical data. This is less time consuming than estimating the tangential angles needed for the straight tube approximation. This method is preferable to the straight tube approximation method because calculation of the AIF/VOF using the phase information including the geometry factor can occur without user interaction. The user would track through the magnitude images to select a seed to grow the AIF/VOF region. This would simplify the perfusion modelling process and make it more practical for the clinical environment. However, current results indicate that it is less reliable than the straight tube approximation method.

In this method the susceptibility map needed as input to the magnetic field shift 
calculation is not measured directly. The map is estimated from a scan, which is part of the standard routine. This has the advantage that it does not increase the scan time. The smoothing, resampling and region growing methods applied to these images can impact the accuracy of the estimated susceptibility. Future work in this area could lead to a more optimal method to create realistic susceptibility maps.

The pixel location mapping between the dynamic series and the geometry factor map was determined using the image position and image orientation given in the DICOM header of the image. It is possible that some patient motion occurred between the acquisition of the approximately transverse images of the dynamic scan and the approximately sagittal images used to generate the susceptibility map. A 3D registration algorithm which maps the sagittal image to the transverse image would be an essential part of the clinical implementation of this technique. The same would be true of a susceptibility map method, and is thus not a flaw in the method but simply a practical necessity of any correction technique.

\subsection{Conclusions}

The simulation results presented in this chapter demonstrate that the dipole approximation method can be used to estimate the phase accumulation for objects of known geometry with parameters similar to those seen in DCE-MRI. It was observed that the simulation error was generally less than $1 \%$ and that the most accurate results can be achieved when the volume of the simulated vessel is relatively small compared with the background volume.

The experimental phantom results presented in this chapter demonstrate that the dipole approximation method can be used to calculate the phase accumulation for a phantom vessel with a complex geometry.

With the validity of the method well established, it was also possible to prove that 
the straight tube approximation provides good accuracy for predicting the geometry factor for curved vessels typically seen in the superior sagittal sinus. An examination of simulated tori also suggests that greater phase enhancement can sometimes be seen outside the vessel and that an appropriate geometry factor must be applied if this data is to be interpreted correctly.

The method was also applied to clinical data as a proof of principle. This avenue merits future work as the method could potentially be applied as an automatic correction directly on the scanner with minimal user intervention. Given the success with the phantom experiment, optimizing the processing of the clinical data will likely improve the method.

For the studies with human volunteers presented in the following two chapters, a straight or slightly curved portion of the vessel is used in the analysis to calculate the AIF/VOF, as it appears to provide a more consistent result than using a susceptibility map generated from clinical data. 


\section{Chapter 6}

\section{Brain Tumour Study}

\subsection{Introduction}

There has been considerable interest for many years in the question of how to measure tissue perfusion parameters accurately and reproducibly with MRI but the solution to this problem has remained elusive. The main difficulty with these studies is the determination of a reliable arterial input function (AIF). Recently, Parker et al. [38] presented a population averaged AIF which, for many cases, is a reasonable approximation to the AIF for a specific subject. However, it would be preferable to accurately measure the AIF for each patient directly, since deviations from the population AIF may occur in specific subject groups due to, for example, underlying pathology.

The traditional approach to measuring the AIF with MRI uses the magnitude of the MRI signal, $|S|$, as a measure of the concentration of the gadolinium-based contrast agent $(\mathrm{CA})$, where a linear relationship between $|S|$ and the concentration of the CA, $[\mathrm{CA}]$ is often assumed. The assumption of linearity works well for low concentrations such as found in the tissue capillary bed but it is a poor assumption in larger vessels where the $[\mathrm{CA}]$ can be much higher. An additional problem with measuring [CA] in large vessels using $|S|$ is that it is quite susceptible to in-flow effects. On the other hand, the phase of the MR signal, $\phi$, is not affected by in-flow 
effects [49] (see also Chapter 3) and is known to be linear with [CA], even for the large concentrations that are expected in the arteries and veins [49]. This linearity was also verified here using a phantom and the results are presented in Chapter 4 of this thesis. These properties of $\phi$ have motivated us to use a $\phi$-derived AIF, AIF ${ }_{\phi}$, in DCE-MRI experiments rather than the conventional $|S|$-derived $\mathrm{AIF}, \mathrm{AIF}_{|S|}$, for a group of patients with brain tumours.

Intracranial tumours provide an excellent model for studying the proposed $\phi$-based DCE-MRI for a variety of reasons including the facts that the SNR for the head coil is high compared with the body coil and motion can be more easily controlled in the head than in the torso. The resulting $\mathrm{AIF}_{\phi}$ s compare favourably with the population AIF of Parker et al. [38].

For cerebral perfusion studies of healthy subjects the CA will pass through the brain tissue with none of it leaking out of the vasculature into the brain tissue since it cannot pass through a healthy blood brain barrier (BBB). In this case, the AIF and the venous output function (VOF) can be expected to be very similar in shape and the area under the two curves should be equal since this area is proportional to the amount of CA passing through the system in both cases. Small differences in the two functions are expected since 1) the passage of the CA through the tissue will cause a time delay for the VOF relative to the AIF and 2) the VOF is expected to be broader than the AIF since there will be a multitude of different paths and path lengths through the capillary beds of the tissue. Nevertheless, the AIF and VOF for the brain of a healthy subject are expected to be similar. This is a useful correspondence since it is often easier to measure the VOF than the AIF; this was certainly true for the study presented in this chapter.

In patients with brain tumours, the similarity between the AIF and the VOF can be expected to be lower than for healthy subjects since some of the CA will leak out of the vasculature in the tumour where the $\mathrm{BBB}$ is compromised. However, 
most of the blood entering the brain will normally leave the brain without passing through the tumour so that, even for brain tumour patients, the shape of the VOF should be reasonably similar to that of the AIF, although the area under the curve can be expected to be a bit lower. In the study presented in this chapter, the AIFs and the VOFs derived from the phase of the MR signal compared favourably both with the population AIF given by Parker et al. [38] and with each other. These two functions were then used to calculate the perfusion parameters for a small cohort of brain tumour patients with encouraging results.

In this chapter, the clinical application of the $\phi$ based DCE-MRI technique, developed and validated in the previous chapters, to the measurement of perfusion parameters in intracranial tumours is presented. In Section 6.2 the method used for determining the $\mathrm{AIF}_{\phi}$ and $\mathrm{VOF}_{\phi}$ is presented and the results obtained with these functions in an ongoing clinical study of perfusion in brain tumour patients are given in Section 6.3. A discussion of the success of the study and the relative merits of the compared techniques is presented in Section 6.4. Finally, conclusions concerning this and future studies are provided in Section 6.5.

\subsection{Methods}

\subsubsection{The Clinical Protocol}

The patient data presented in this chapter were acquired as part of an ongoing study at the Ottawa Hospital, Ottawa, Canada. This is a prospective cohort study carried out from Sept 2008 to the present at the Civic Campus of the Ottawa Hospital. All patients presenting with a newly diagnosed brain lesion compatible with a glioma are asked to participate, except for patients with contraindications for MR, impaired renal function, or those who are pregnant. Informed consent by the patient or legal guardian 
or authorized representative of each patient is obtained before enrollment in the study. The aim is to recruit 25 patients with cerebral gliomas. Each patient is imaged presurgery, post-surgery and post-radiation depending on the treatment received by the specific patient, which is given in accordance with the protocol of the hospital. These patients will be followed clinically for two years after initial treatment to assess the clinical outcome. For this thesis the input and output functions from 28 studies were investigated. The perfusion maps for 10 pre-surgery exams are also analyzed. $T_{1}$ weighted DCE-MRI imaging is performed using an injection of a gadolinium-based CA, Magnevist (®Bayer Healthcare). A dose of $0.1 \mathrm{mmol} / \mathrm{kg}$ is injected as a bolus using a power injector, followed by a saline flush to ensure reproducibility.

All image data presented here were obtained using a 1.5 T Siemens Symphony scanner with CP Head Array coil, spine coil and neck coil (Siemens Medical Solutions, Erlangen, Germany). A double echo, 2D spoiled gradient echo (SPGR) sequence with $T_{R}=46 \mathrm{~ms}, T_{E}=(2.06,5.48) \mathrm{ms}$ and $\alpha=90^{\circ}$ was used for the dynamic series. The matrix size was $96 \times 128$ with a field of view (FOV) of $172.5 \times 230 \mathrm{~mm}^{2}$ leading to a resolution of $1.8 \times 1.8 \mathrm{~mm}^{2}$. Five transverse slices, each with a thickness of 5.5 $\mathrm{mm}$ were acquired, including four that covered the tumour volume and an extra slice placed through the carotid artery in a location where the artery is straight and as parallel as possible to the main magnetic field. The temporal resolution was $2.16 \mathrm{~s}$ and 100 time points were acquired for a total scan time of 3.6 minutes. The CA was injected 1 minute after the beginning of the scan. In addition to the conventional magnitude data, the complex $k$-space data was saved as well. The slice locations and relevant anatomy are shown in Fig. 6.2.

$T_{1}$ maps were obtained both before and after the dynamic series acquisition using the variable flip angle method described in Section 2.8.4. For these measurements a 3D SPGR sequence was used with the parameter settings: $T_{R}=50 \mathrm{~ms}, T_{E}=2.16$ ms and $\alpha=10^{\circ}, 20^{\circ}, 40^{\circ}, 70^{\circ}$. The matrix size was $(96 \times 128 \times 20)$ with the same 
in plane resolution as the dynamic series and a slice thickness of $2.75 \mathrm{~mm}$. $T_{1}$ was evaluated pixel by pixel by fitting the signal intensities for these four flip angles to Eq. 2.119. These maps were then resampled to correspond with the slice locations of the dynamic series.

A 2D method is used here to allow for improved time resolution as compared to a 3D approach. This type of acquisition also allows for multiple slice blocks to be prescribed such that, in addition to the slices through the tumour, a slice through the neck can also be added to the protocol. This provides the opportunity to measure the $\phi$ versus time in the arteries of the neck. These vessels, which have different diameters, can be compared as an in vivo measure of the effect of vessel size on the measured $[\mathrm{CA}]$. In addition, the input from a slice through the patient's neck is compared to the output from the tumour slices to answer the question of whether a shifted VOF, which is easier to measure, can be used in place of an AIF to obtain the same perfusion parameters.

Image analysis was performed offline using custom software written in IDL@ (ITT Visual Information Solutions, Boulder, CO) to reconstruct the raw data and manipulate images.

\subsubsection{Signal Phase Extraction}

Although the combined signal magnitude from phased array coils is provided directly by the MRI scanner, the combined signal phase was not available for this sequence for this version of the MRI scanner software. For this reason, the phase information from each coil was combined offline. The method for combining signals from different coils, developed in Appendix A, involves estimating a coil sensitivity to be used when combining the complex data. The coil sensitivities were calculated from the background signal using Eq. 2.66. The mean of the background magnitude signal of a $10 \times 10$ block of pixels in the corner of each image in the dynamic series was used. 


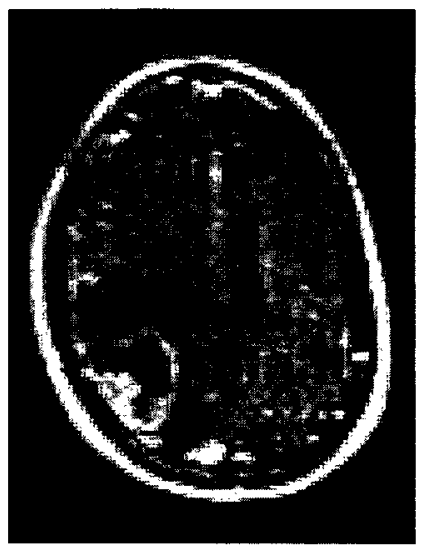

a)

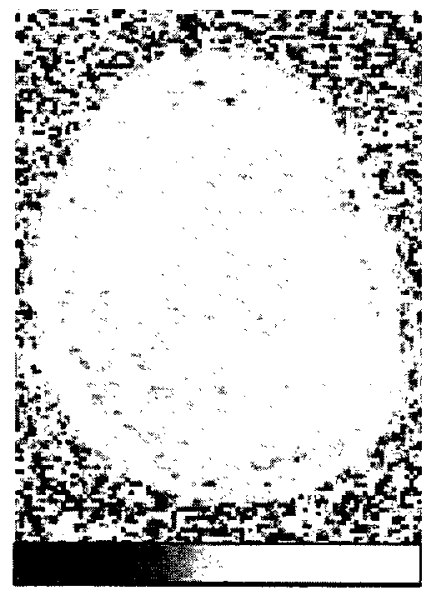

b) $-\pi$

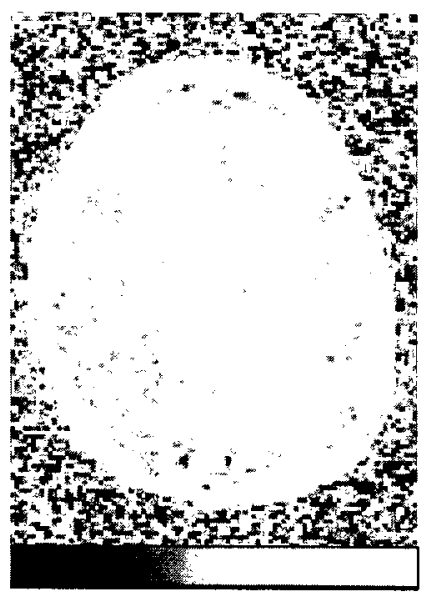

c) $-\pi$

Figure 6.1: Typical DCE-MRI images for the same patient and slice; a) a $|S|$ image taken at maximum [CA] in the SSS b) a baseline $\phi$ image c) a $\phi$ image taken at maximum $[\mathrm{CA}]$ in the SSS.

Finally, the cumulative phase change over time is calculated for the entire dynamic series. Typical magnitude and phase images are presented in Fig. 6.1.

The $\mathrm{AIF}_{\phi}$ and $\mathrm{VOF}_{\phi}$ used in calculating the perfusion maps were calculated using the $\phi$ data acquired with a $T_{E}=5.48 \mathrm{~ms}$ since this, the longer of the two echo times in the dual echo sequence, provides more $\mathrm{SNR}_{\phi} . \mathrm{VOF}_{\phi} \mathrm{s}$ derived using the $\phi$ data acquired with a $T_{E}=2.06 \mathrm{~ms}$ were also calculated for comparison with the longer echo time $\mathrm{VOH} \phi$ s, bus perfusion maps were not generated.

\subsubsection{Determination of Input/Output Functions}

\section{ROI Selection}

A large region of interest (ROI) was drawn around each vessel of interest in all slices, including the neck slice. In the neck slice, ROIs were drawn for the carotid arteries, vertebral arteries, and internal jugular vein. In the tumour slices, only the superior sagittal sinus (SSS) was included.

To correct for motion, the maximum magnitude pixel intensity was determined from within the ROI for each point in the dynamic series. For the $\mathrm{VOF}_{|S|}$, all pixels 
with magnitudes within $\sigma_{g}$ (as defined in Section 2.5) of that maximum were kept to form the mean of the ROI for each image in the dynamic series, for each $T_{E}$.

The signal at $T_{E}=0 \mathrm{~ms}, S_{0}$, was then calculated from

$$
S\left(T_{E i}\right)=S_{0} e^{-T_{E i} / T_{2}^{*}}
$$

where $T_{E i}$ is the $i^{\text {th }}$ echo time and $S\left(T_{E i}\right)$ is the magnitude signal obtained at that echo time using the dual echo SPGR sequence introduced in the previous subsection. There is an increase in the peak of $\mathrm{VOF}_{|S|}$ when using $S_{0}$ as compared to using the magnitude image acquired at either echo time individually.

The concentration was then calculated using Eq. 2.126 from Section 2.8.5, assuming a $T_{1}$ for blood of $1250 \mathrm{~ms}$ [35]. A $T_{1}$ map cannot be used to measure the $T_{1}$ inside a vessel because the intra vessel signal obtained using an SPGR sequence is corrupted by blood flow and is not properly described by Eq. 2.81. For AIF ${ }_{\phi}$ and $\mathrm{VOF}_{\phi}$, pixels with magnitudes within $10 \sigma_{g}$ of the maximum were included. The larger acceptance range was chosen after testing a range of values. The $\phi$ data for these pixels was then converted to concentration using Eq. 2.114. The method used to determine the geometry factor required for this computation is described in the next section.

\section{Vessel Angle Determination}

To determine the angle of the SSS at each slice through the tumour with respect to $\mathbf{B}_{\mathbf{0}}$, a one- or two-pixel ROI was drawn at the most central pixel in the SSS for each slice of a post contrast 3D image (acquired as part of the bookend method). The image position and orientation, provided in the image file header (DICOM format), were used to map the location of the ROI onto the patient/magnet coordinate system. Next, a $2^{\text {nd }}$ order polynomial fit was applied to the data. For each slice location, the tangent of the curve (first order derivative) was used to calculate the angle between 


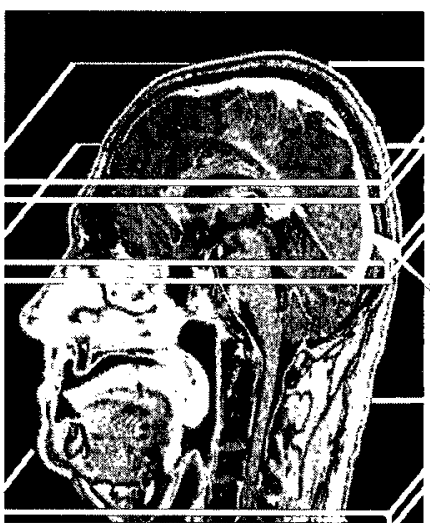

a)

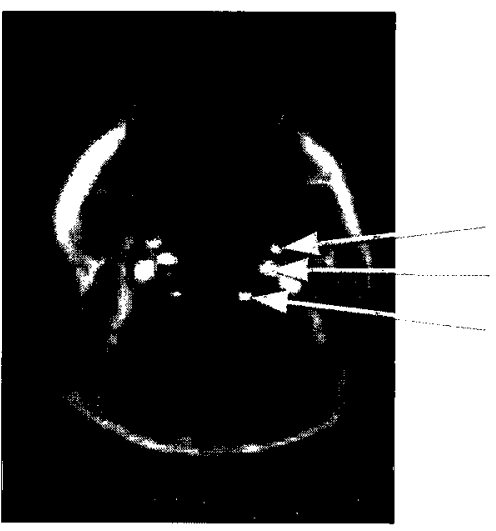

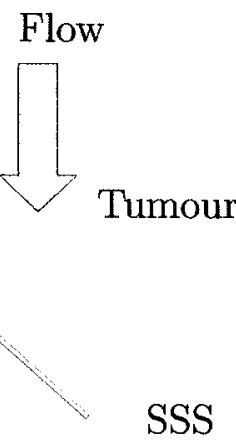

ECA ICA VA

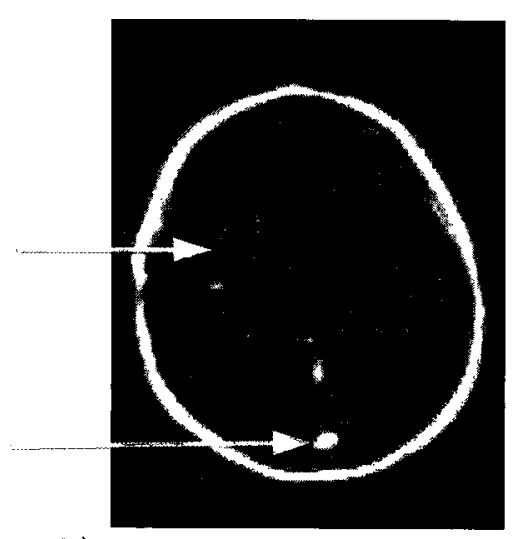

b)

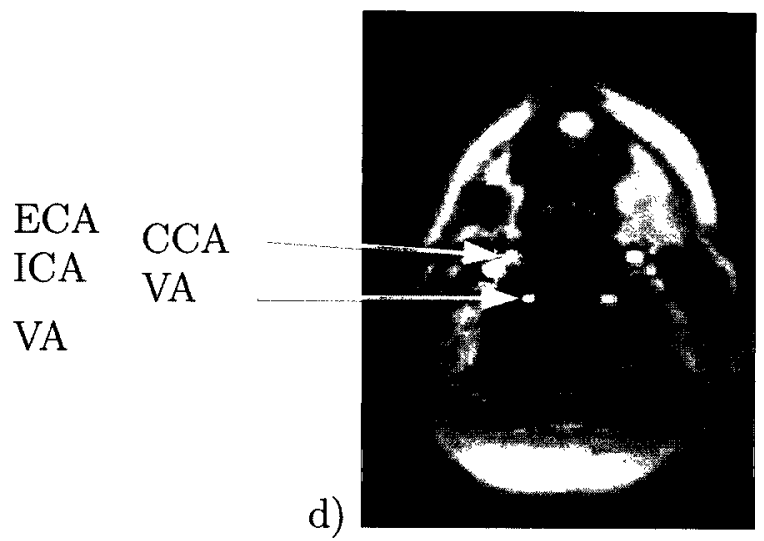

Figure 6.2: Schematic of the 2D DCE-MRI protocol applied to the brain tumour study. a) A sagittal view of the brain shows the approximate slice location in the exam. Note that the blood flow through the SSS is in the superior to inferior direction. b) A typical image slice through the tumour and SSS. c) A typical "upper" neck slice showing the internal carotid artery (ICA), the external carotid artery (ECA) and the vertebral artery (VA). d) A typical "lower" neck slice showing the common carotid artery (CCA) and the vertebral artery (VA). 
the vessel and the main magnetic field.

In the case of the slice through the neck, a 3D image covering this region was not obtained, and so a different angle determination method was used. Maximum intensity projection images in the coronal and sagittal planes were used to measure the angles of the vessels at the location of the neck slice. These vessels were much straighter that the SSS, thus curvature was not an issue.

\subsubsection{Determination of Tissue Concentration}

The tissue concentration curves were determined pixel-by-pixel by using the bookend method described in Section 2.8.5 using in house software written in IDL. Since a double echo SPGR sequence was used, $S_{0}$ was calculated in the same way as described above. When $S\left(T_{E 1}\right)<S\left(T_{E 2}\right)$, it was assumed that the data was too noisy and so the average of the two signals was used for $S_{0}$ in this case. The results were constrained to be between $0 \mathrm{mM}$ and $25 \mathrm{mM}$.

\subsubsection{The Perfusion Map Method}

Once the pixel-by-pixel tissue concentration maps and the input and output functions were calculated they were imported into a perfusion modelling software suite for the calculation of the perfusion parameters. Perfusion modelling was performed on ten pre-surgery cases using an imaging software suite called NordicIce ${ }^{\circledR}$ (NordicImagingLab, Bergen, Norway). This program uses the model described by Eq. 2.34 and the method of Murase [53] to extract the perfusion parameters $K^{\text {trans }}, v_{p}$ and $v_{e}$. This program uses a default haematocrit ratio of 0.45 .

The resulting maps of the perfusion parameters, which were calculated pixel-bypixel, were saved and imported into ImageJß (National Institute of Health, USA) for further analysis. To facilitate comparison between the studies, ROIs were drawn 
in the tumour regions for each case. ROI selection was performed by an Ottawa Hospital radiology resident. For tumours with areas of high enhancement, several square ROIs were placed in the enhancing outer ring of the tumour, avoiding areas of necrosis. For tumours with no high enhancing regions, the ROI was placed centrally inside the tumour. The combined mean and SD of the perfusion parameters were compared for each different input function using linear regression, on Origin $5.0 ®$ (Microcal Software Inc, Northampton, MA, USA)

\subsection{Results}

The preliminary results of the glioma study, described above, are presented in this section. First, the AIFs and VOFs will be presented for 28 cases. Although the desired results from a DCE study are the perfusion parameter maps, in establishing the validity of the technique it is useful to study the accuracy of the AIFs and VOFs. This is an important intermediate step. From this, it is possible to determine the effect of different acquisition parameters on the AIFs and VOFs and to ensure that the results are reasonable. Specifically, the $\mathrm{VOF}_{|S|}$ and $\mathrm{VOF}_{\phi}$ will be compared, as will the $\mathrm{AIF}_{\phi}$ and $\mathrm{VOF}_{\phi}$. The effect of angle correction, vessel size and $T_{E}$ on the $\phi$-derived results will also be assessed. These effects must be understood in order to establish an optimal clinical protocol. Finally, perfusion maps for ten studies using $\mathrm{VOF}_{|S|}, \mathrm{AIF}_{\phi}$ and $\mathrm{VOF}_{\phi}$ will be presented. $|S|$-derived AIFs were not computed, as a single slice (i.e. the slice containing the carotid artery) cannot provide reasonable magnitude measurements, owing to blood flow, and the arteries in the slices containing the tumour are small and therefore susceptible to partial volume and signal leakages errors. 


\subsubsection{Input and Output Functions}

To simplify the comparison between different input and output functions, some basic parameters are used to characterize the time course (see Fig. 6.3). First, the peak height was defined as the maximum [CA] in the time series. This is an easy way to compare the scaling between methods. Second, the area under the curve (AUC) provides information about the volume of CA. For this dataset, the area under the peak $\left(\mathrm{AUC}_{\text {peak }}\right)$ was calculated. The time over which this area was calculated was selected to include the whole peak but avoid the recirculation peak as much as possible; it was $24 \mathrm{~s}$ for the AIFs and $32 \mathrm{~s}$ for the VOFs. These ranges are different because the VOF is more dispersed than the AIF. The third parameter was the washout concentration, which was defined as the mean concentration over $24 \mathrm{~s}$, starting $65 \mathrm{~s}$ after the peak. This range was chosen to avoid the recirculation peak and average over an approximately flat portion of the time course. The population mean and SD for the different input and output function characterization parameters are listed in Table 6.1, along with the corresponding parameters for a population AIF from the literature, scaled to the same dose [38]. The $\mathrm{AIF}_{\phi}$ and $\mathrm{VOF}_{\phi}$ parameters are in good agreement with those for the population AIF but, as expected, the $\mathrm{VOF}_{|S|}$ values are quite different. The $\mathrm{VOF}_{\phi}$ values given in the table are geometrically corrected as discussed in the next subsection. Note that all results presented in this section are for whole blood.

\section{Geometry Correction}

The angle between the SSS and $\mathbf{B}_{\mathbf{0}}$ was, on average $17^{\circ}$, with a SD of $14^{\circ}$, for all slices. This is a sufficiently large angle to justify the angle correction introduced in Section 2.8.2. Figure 6.4 shows an example $\mathrm{VOF}_{\phi}$ before and after angle correction for each slice. This case, although particularly dramatic, illustrates the concept. Most corrections applied were less dramatic. For this example the angles were $22^{\circ}, 29^{\circ}, 38^{\circ}$ 


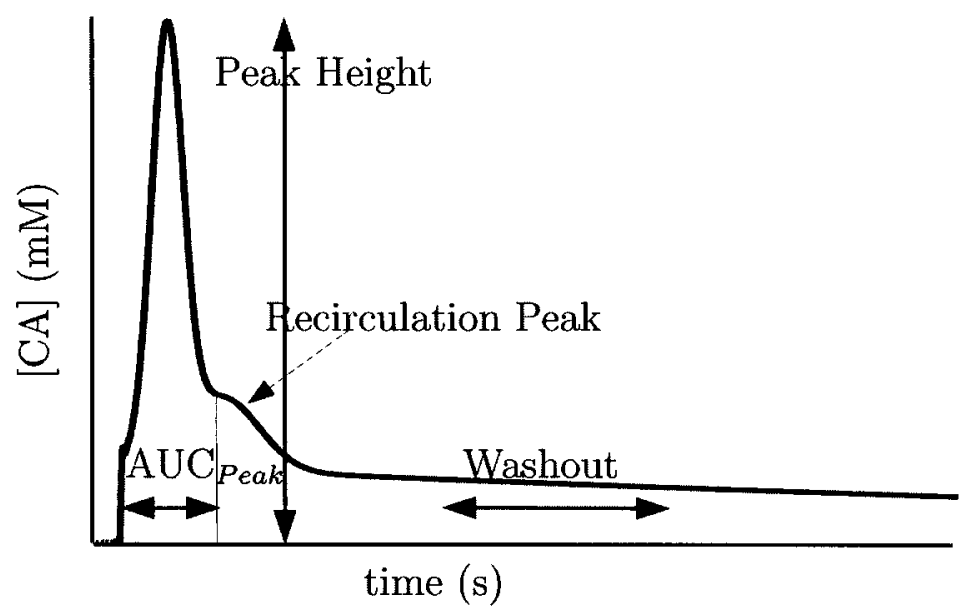

Figure 6.3: Schematic of input/output function characterization parameters, peak height, area under the peak $\left(\mathrm{AUC}_{\text {peak }}\right)$ and washout.

Table 6.1: Summary of average AIF and VOF characterization parameters. The results are presented as mean (SD). There were twenty-cight studies included in each mean except for $\mathrm{AIF}_{\phi}$ where twenty-five cases were used, three cases were excluded because the neck coil was not used and thus there was not sufficient SNR in the neck slice to analyze this data.

\begin{tabular}{|cccc|}
\hline Set Up & Peak $(\mathrm{mM})$ & $\mathrm{AUC}_{\text {peak }}(\mathrm{mM} \mathrm{s})$ & Washout $(\mathrm{mM})$ \\
\hline $\mathrm{AIF}[38]$ & 7.1 & 58 & 1.1 \\
$\mathrm{AIF}_{\phi}$ & $8.3(2.6)$ & $77(24)$ & $1.1(0.5)$ \\
$\operatorname{VOF}_{\phi}\left(T_{E}=2.06 \mathrm{~ms}\right)$ & $6.1(2.2)$ & $68(26)$ & $0.92(0.53)$ \\
$\operatorname{VOF}_{|S|}$ & $1.6(0.9)$ & $23(13)$ & $0.43(0.23)$ \\
$\operatorname{VOF}_{\phi}\left(T_{E}=5.48 \mathrm{~ms}\right)$ & $6.2(2.1)$ & $70(22)$ & $0.98(0.38)$ \\
\hline
\end{tabular}




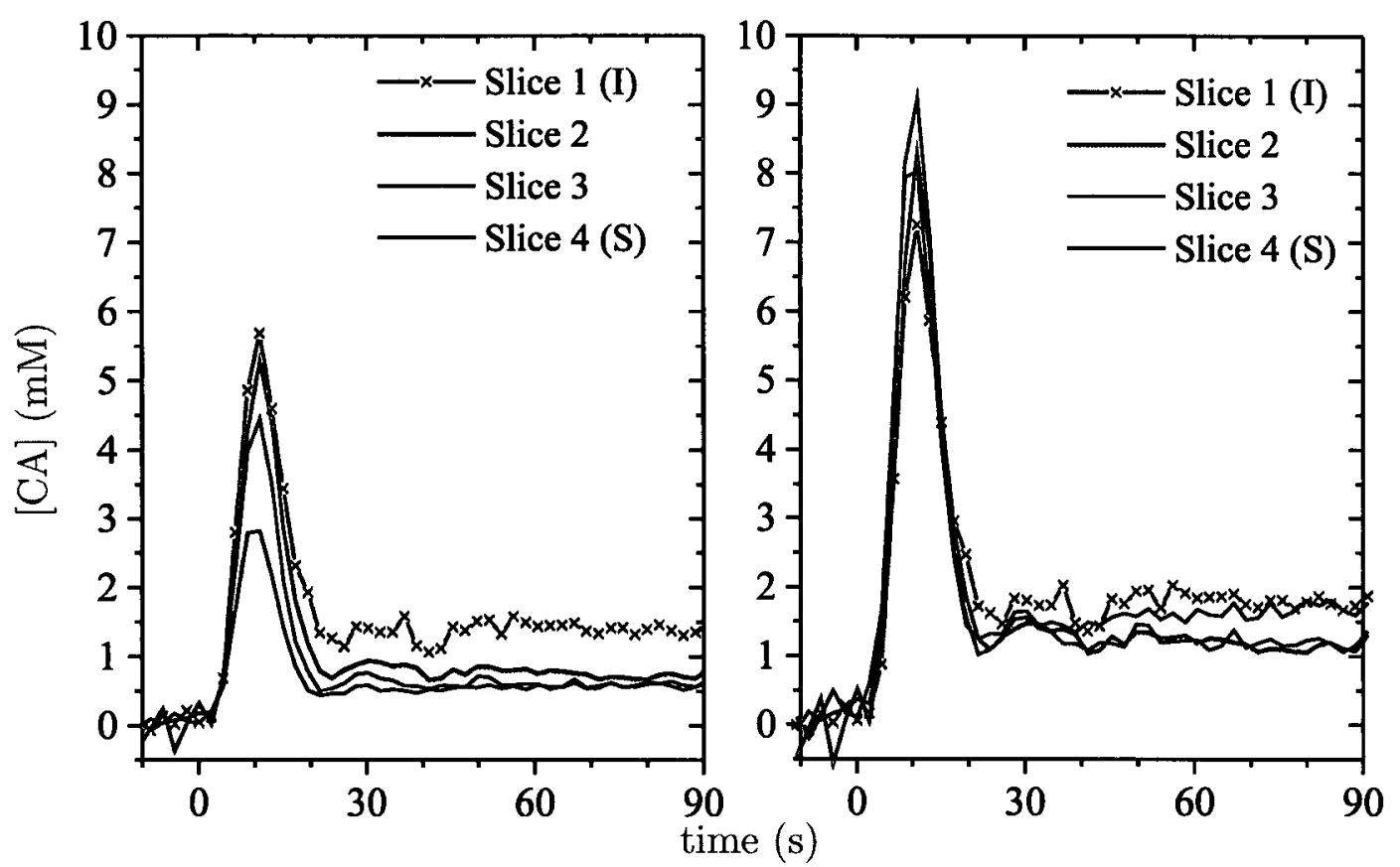

Figure 6.4: A typical $\mathrm{VOF}_{\phi}$ measured in the SSS before and after incorporating the geometry factor into the phase to $[\mathrm{CA}]$ conversion. Slices order from most inferior (I) to most superior (S).

and $41^{\circ}$ for slices 1 to 4 (inferior to superior), respectively.

The orientation of the neck vessels with respect to $\mathbf{B}_{\mathbf{0}}$ was also investigated (as described earlier). The angle between the neck vessels and $\mathbf{B}_{\mathbf{0}}$ was, on average $11^{\circ}$, with a SD of $9^{\circ}$, for all vessels.

\section{Vessel Size}

The image slice through a patient's neck contains several vessels (see Fig. 6.2) and thus provides the opportunity to compare the results for different vessel sizes. The vessels in the neck slice were grouped into carotids and vertebral arteries. The patients' neck slices were grouped into two locations; a lower slice containing the common carotid (15 cases) or a higher slice where the common carotid had bifurcated into internal and external carotid arteries (10 cases). For the lower neck slices, the AIFs measured from the two types of vessels (common carotid and the vertebral arteries) were compared 
(15 cases). There was a difference in peak height of $1.1 \pm 0.2 \mathrm{mM}$, which was found to be statistically significant using a paired t-test $(p=0.0002)$. Thus, it was decided to use only the larger vessels (carotids) for the perfusion modelling in this case. It is interesting to note that there was no significant difference found in the washout level $(p=0.15)$. The upper neck slices did not show a significant difference for any of the AIF characterization parameters $(p>0.18)$, thus it was decided to average all arteries in these slices.

\section{Echo Time}

As mentioned above, $\mathrm{VOF}_{\phi}$ was calculated using the $\phi$ data acquired with a $T_{E}=5.48$ ms; the longer of the two $T_{E}$ 's in the duel echo SPGR sequence. The $T_{E}$ corresponds to the length of time for which $\phi$ can accumulate and so for a longer $T_{E}$ the $\mathrm{SNR}_{\phi}$ is higher. It is useful to know how short $T_{E}$ can be while still measuring accurate input and output functions since a shorter $T_{E}$ allows for an increase in time resolution. To investigate the effect of $T_{E}$ on the accuracy of the input and output function, $\mathrm{VOF}_{\phi}$ was also calculated using the $\phi$ data acquired with the shorter $T_{E}\left(T_{E}=2.16 \mathrm{~ms}\right)$ for comparison.

For two cases, the short $T_{E}$ derived $\mathrm{VOF}_{\phi} \mathrm{s}$ were unreasonable, possibly due to signal leakage and thus 26 cases were compared. There was no statistically significant difference between any of the output characterization parameters $(p>0.78$ using a pairwise t-test). An F-test revealed that there was no difference in the variance for peak height $(p=0.85)$ or $\mathrm{AUC}_{\text {peak }}(p=0.41)$ and only a small difference in the variance for the washout $(p=0.08)$. It is expected that the data acquired using the longer $T_{E}$ would have less variation that the data acquired using the shorter $T_{E}$. This expected difference is not apparent, probably because the patient-to-patient variation is higher than the difference in $\mathrm{SNR}_{\phi}$ for the two $T_{E}$ 's. To mitigate this, the SD of the washout within each patient's $\mathrm{VOF}_{\phi}$ was calculated. The mean value of the SD 


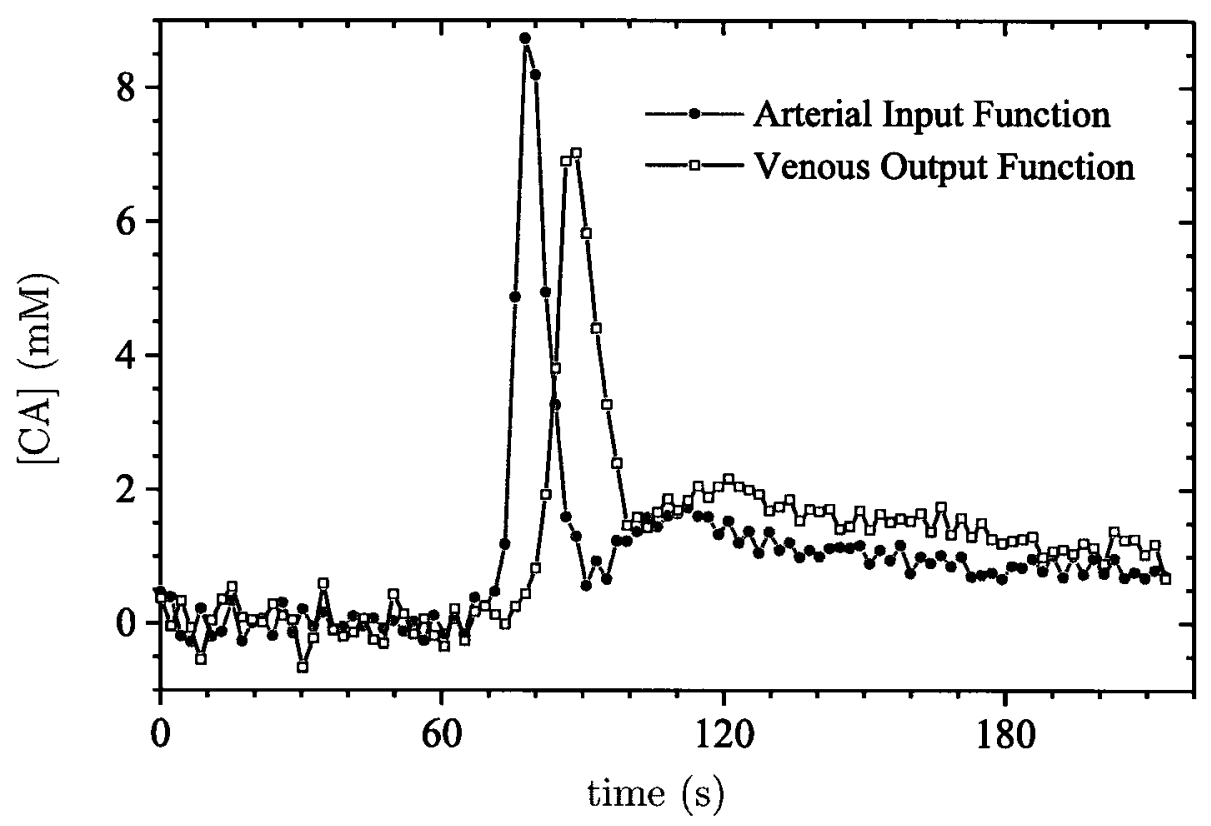

Figure 6.5: Typical $\mathrm{AIF}_{\phi}$ and $\mathrm{VOF}_{\phi}$ curves.

for the short and long $T_{E}$ 's was $0.19 \mathrm{mM}$ and $0.09 \mathrm{mM}$, respectively. A pairwise t-test showed a significance between these means $(p=0.001)$. Therefore, the data presented here suggests that a difference in the SD of the washout values (i.e. SNR in the curve) does exist as was observed in Chapter 4.

\section{Comparison of $\mathrm{AIF}_{\phi}$ and $\mathrm{VOF}_{\phi}$}

The difference between the $\phi$-derived input and output functions was assessed. A typical case is presented in Fig. 6.5. A delay of between 3-4 time points (6.5-8.6 s) between the $\mathrm{VOF}_{\phi}$ and $\mathrm{AIF}_{\phi}$ was observed and some dispersion is also apparent. A pairwise t-test revealed that the peak height for $\mathrm{AIF}_{\phi}$ was significantly higher $(p<0.0001)$ than the peak height for $\mathrm{VOF}_{\phi}$, with a mean difference of $1.92 \pm 0.07$ $\mathrm{mM}$. The $\mathrm{AUC}_{\text {peak }}$ and washout were not significantly different at $p=0.15$ and $p=0.29$, respectively. 


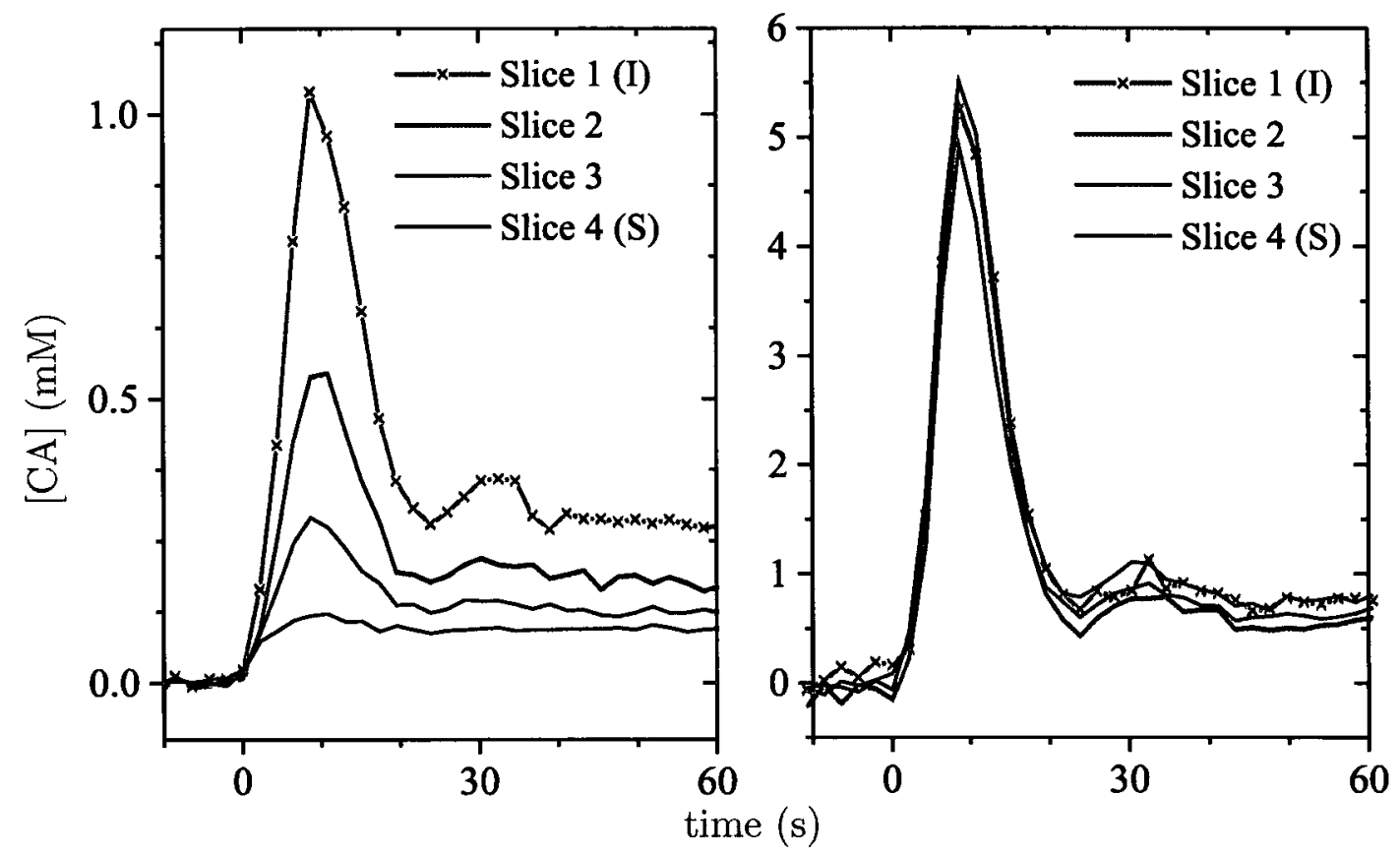

Figure 6.6: Typical $\mathrm{VOF}_{|S|}$ and $\mathrm{VOF}_{\phi}$ curves for each slice through the tumour. Slices order from inferior (I) to superior (S). Note that the graphs do not show the full measured time course.

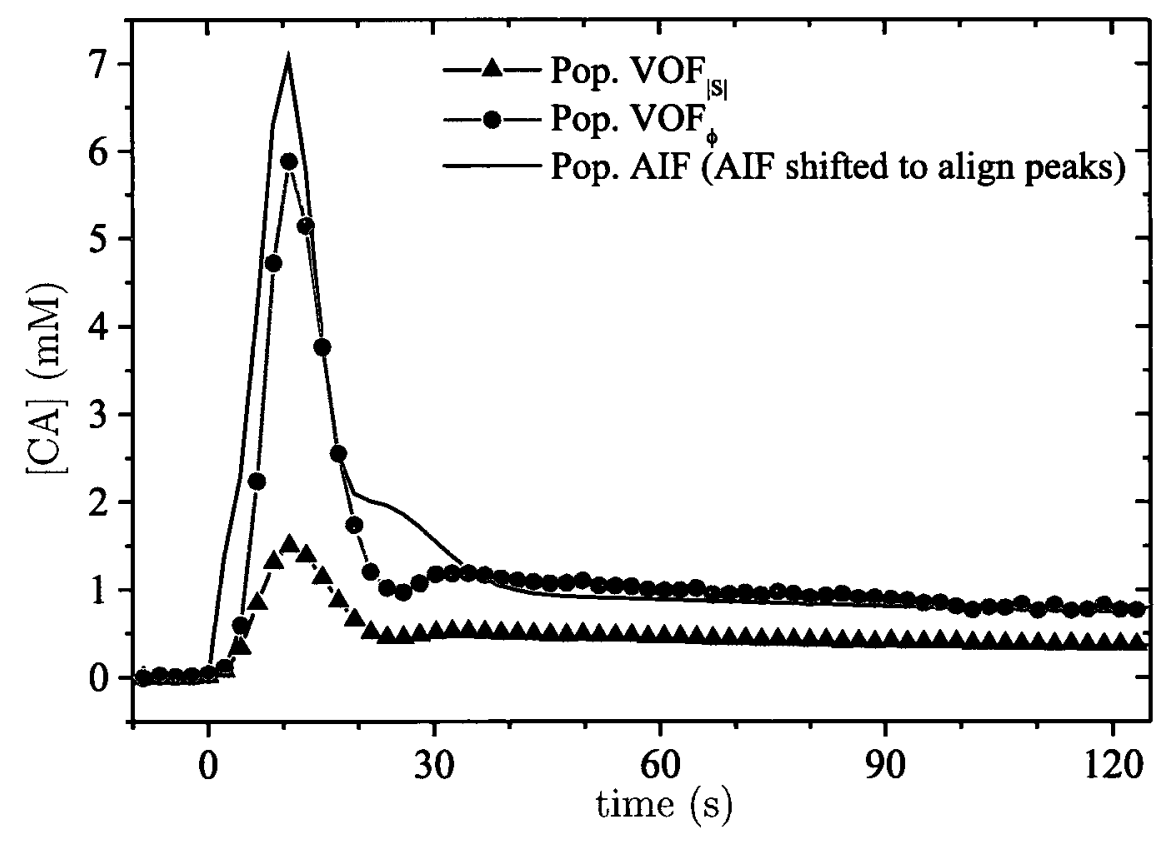

Figure 6.7: $\mathrm{VOF}_{|S|}$ and $\mathrm{VOF}_{\phi}$ averaged over 28 cases compared to a population AIF from the literature [38], which is time-shifted so that the peaks coincide. 


\section{Comparison of $\mathrm{VOF}_{|S|}$ and $\mathrm{VOF}_{\phi}$}

One of the main objectives of this chapter is to compare the inputs and output functions derived using $\phi$ to those which would be derived from a typical DCE study using current techniques (i.e. using $|S|$ ). The $[\mathrm{CA}]$-versus-time in the SSS was calculated using both $\phi$ and $|S|$. A typical case showing both $\mathrm{VOF}_{|S|}$ and $\mathrm{VOF}_{\phi}$ is plotted in Fig. 6.6 for each slice through the tumour. The peak height of $\mathrm{VOF}_{|S|}$ varied significantly as a function of slice location (1-way ANOVA, $p<0.0001$ ), whereas that of $\mathrm{VOF}_{\phi}$ $\operatorname{did}$ not $(p=0.93)$. Therefore, only the slice with maximum $\operatorname{VOF}_{|S|}$ was used for the final $\operatorname{VOF}_{|S|}$ calculation, whereas the average of all slices was used for the final $\mathrm{VOF}_{\phi}$ calculation (note: some VOFs were rejected if the shape characteristics were unphysical; i.e. if there was no peak or a positive slope in the washout region).

The average $\mathrm{VOF}_{\phi}$ and $\mathrm{VOF}_{|S|}$ are shown in Fig. 6.7. This clearly shows, that in general, there is a large difference in the results obtained using $\phi$ versus $|S|$. A pairwise t-test showed significant differences between $\mathrm{VOF}_{\phi}$ and $\mathrm{VOF}_{|S|}$ for all measurements $(p<0.0001) . \mathrm{VOF}_{\phi}$ also had a smaller COV in peak height, $\mathrm{AUC}_{p e a k}$, and washout than did $\operatorname{VOF}_{|S|}($ F-test, $p<0.01)$.

It is also clear from Fig. 6.7 that the $\phi$-derived results match better with the population AIF measured by Parker et al. [38]. Details of the population AIF are discussed further in Section 6.4. Although the AIF and VOF are not expected to be identical they should have similar characteristics. Note that the population AIF has been time shifted such that the peak coincides with the VOF peak.

\subsubsection{Perfusion Maps}

Perfusion modelling was applied to a sample of ten of the pre-surgery studies using the following input functions: $\mathrm{AIF}_{\phi}, \mathrm{VOF}_{\phi}$ (time shifted) and $\mathrm{VOF}_{|S|}$ (time shifted). 


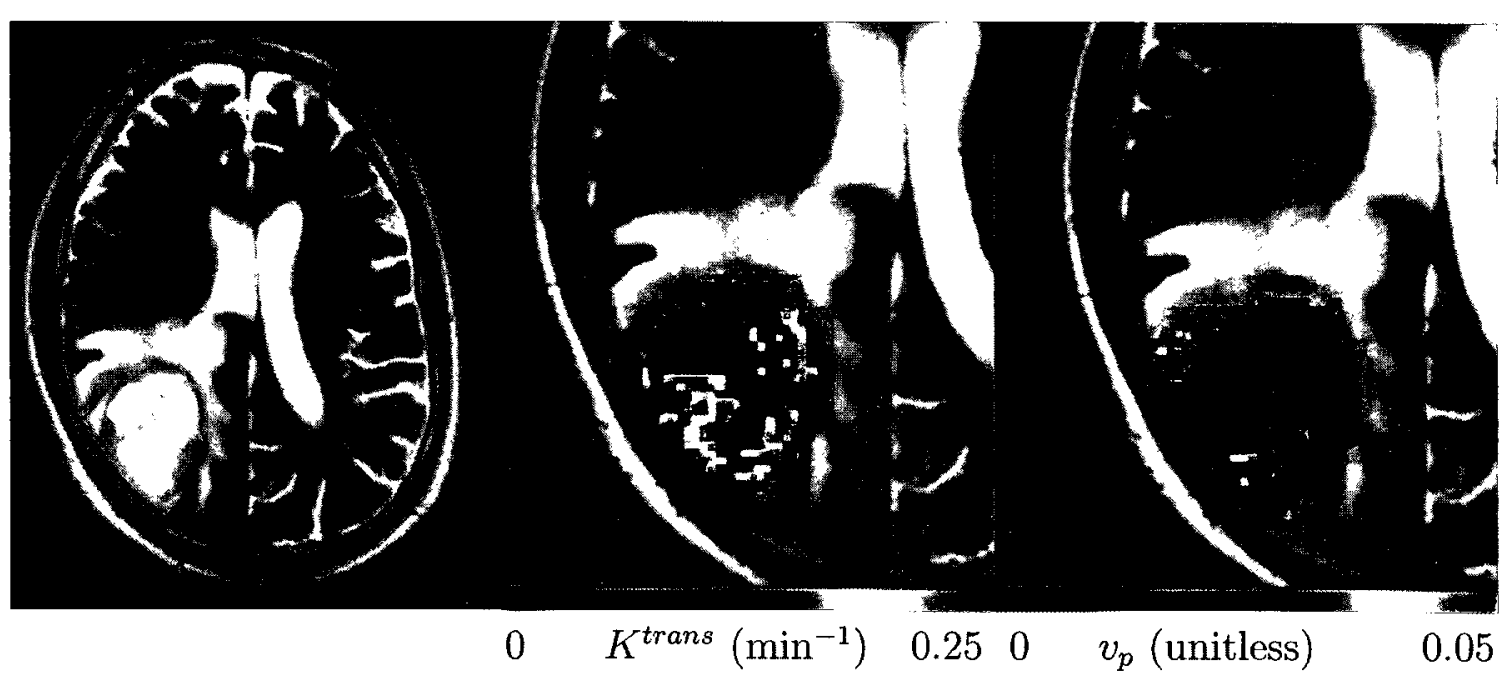

Figure 6.8: $K^{\text {trans }}$ and $v_{p}$ maps computed using $\mathrm{AIF}_{\phi}$ for a grade IV tumour.

The values obtained for $K^{\text {trans }}, v_{p}$ and $v_{e}$ are presented in Tables 6.2 to 6.4 , respectively. Typical $K^{\text {trans }}$ and $v_{p}$ maps, generated using $\mathrm{AIF}_{\phi}$ for a grade IV tumour, are given in Fig. 6.8. Of the ten studies analyzed, two studies for grade II tumours did not have sufficient enhancement for proper fitting using the NordicICE software package; namely studies 6 and 9 . These two studies were not included in the following analysis. The perfusion parameters obtained using $\mathrm{VOF}_{\phi}$ were compared to the

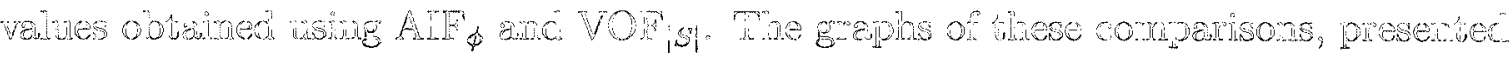
in Fig. 6.9 for $K^{\text {trans }}$ and $v_{p}$. $v_{e}$ produced similar results. An error weighted linear fit was performed for each parameter. It is presented graphically in Fig. 6.9 as a solid line, and analytically in Table 6.5, along with the Pearson's correlation coefficient, $\mathrm{R}$ for all three fit parameters. A dashed line of slope $=1$ is also shown in Fig. 6.9 for comparison. The perfusion results for the grade IV gliomas using all three "input" functions are summarized in Table 6.6 along with results from the literature for comparison. 
Table 6.2: $K^{\text {trans }}\left(\mathrm{min}^{-1}\right)$ values obtained from tracer kinetic modelling using the NordicIce software package where the AIF used in the analysis was $\mathrm{AIF}_{\phi}, \mathrm{VOF}_{\phi}$ (time shifted) or $\mathrm{VOF}_{|S|}$ (time shifted). Note: entries marked $0^{*}$ indicate that NordicIce was not able to perform the fitting due to insufficient tumour enhancement.

\begin{tabular}{|ccccc|}
\hline \multicolumn{4}{|c|}{$K^{\text {trans }}\left(\mathrm{min}^{-1}\right)$} \\
\hline No. & Grade & $\mathrm{AIF}_{\phi}$ & $\mathrm{VOF}_{\phi}$ & $\mathrm{VOF}_{|S|}$ \\
\hline 4 & II & $-0.006 \pm 0.012$ & $-0.01 \pm 0.02$ & $-0.11 \pm 0.18$ \\
6 & II & $0^{*}$ & $0^{*}$ & $0^{*}$ \\
9 & II & $0^{*}$ & $0^{*}$ & $0^{*}$ \\
1 & III & $0.011 \pm 0.018$ & $0.007 \pm 0.008$ & $0.004 \pm 0.010$ \\
2 & IV & $0.038 \pm 0.015$ & $0.056 \pm 0.015$ & $0.25 \pm 0.11$ \\
3 & IV & $0.12 \pm 0.03$ & $0.14 \pm 0.04$ & $0.48 \pm 0.14$ \\
5 & IV & $0.06 \pm 0.03$ & $0.07 \pm 0.03$ & $0.12 \pm 0.08$ \\
7 & IV & $0.16 \pm 0.07$ & $0.20 \pm 0.10$ & $0.9 \pm 0.5$ \\
8 & IV & $0.11 \pm 0.04$ & $0.10 \pm 0.03$ & $0.35 \pm 0.15$ \\
10 & IV & $0.083 \pm 0.012$ & $0.06 \pm 0.04$ & $0.30 \pm 0.13$ \\
\hline
\end{tabular}



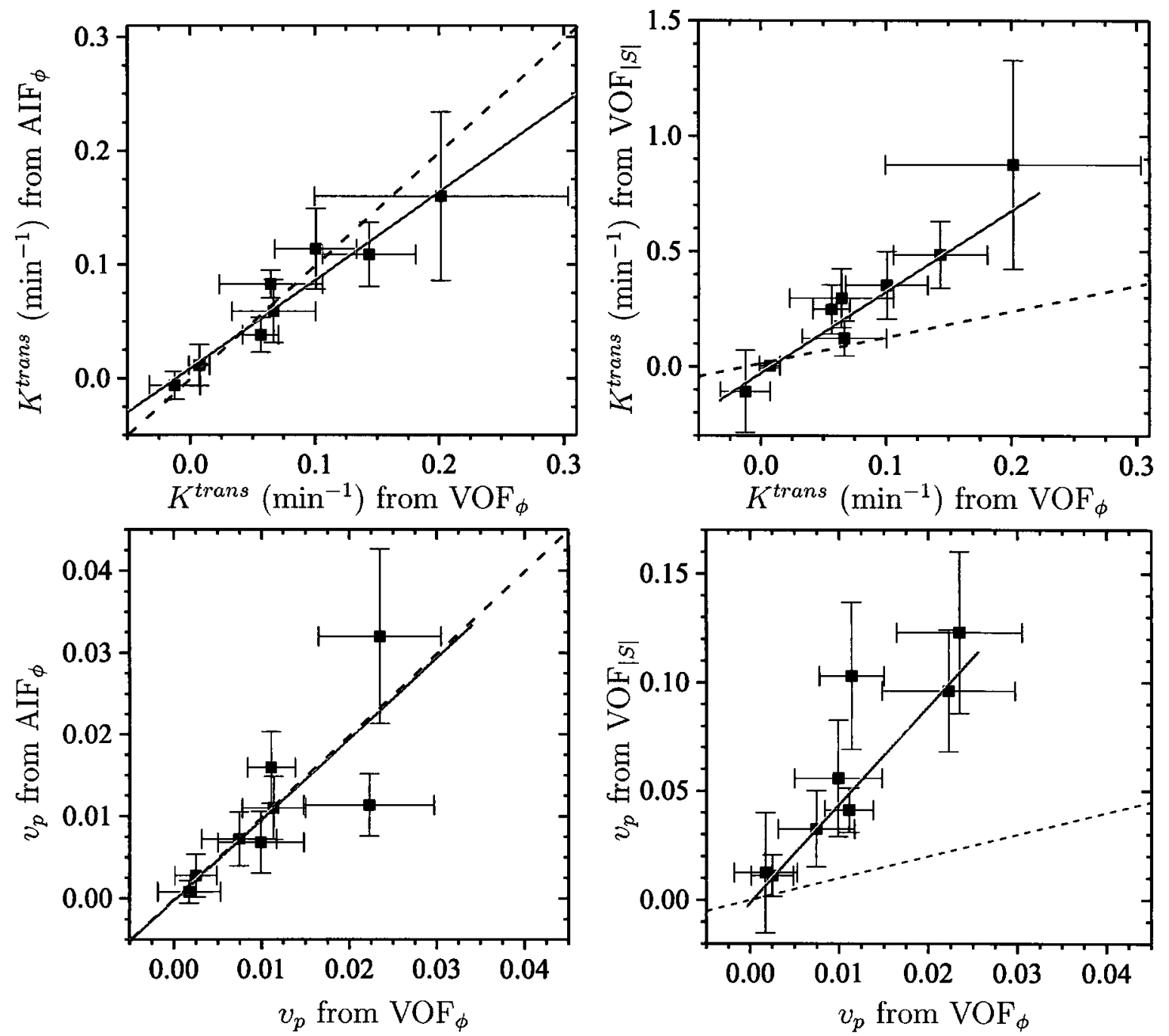

Figure 6.9: $K^{\text {trans }}$ and $v_{p}$ from $\mathrm{AIF}_{\phi}$ versus $\mathrm{VOF}_{\phi}$ and $\mathrm{VOF}_{|S|}$ versus $\mathrm{VOF}_{\phi}$. Error bars on the graph represent one SD in the parameter over the whole ROI for each patient. The dashed line shows the line with slope $=1$. 
Table 6.3: $v_{p}$ values obtained from tracer kinetic modelling using the NordicIce software package where the AIF used in the analysis was $\mathrm{AIF}_{\phi}, \mathrm{VOF}_{\phi}$ (time shifted) or $\mathrm{VOF}_{|S|}$ (time shifted). Note: entries marked $0^{*}$ indicate that NordicIce was not able to perform the fitting due to insufficient tumour enhancement.

\begin{tabular}{|ccccc|}
\hline \multicolumn{5}{|c|}{$v_{p}$} \\
\hline No. & Grade & $\mathrm{AIF}_{\phi}$ & $\mathrm{VOF}_{\phi}$ & $\mathrm{VOF}_{|S|}$ \\
\hline 4 & II & $0.0008 \pm 0.0014$ & $0.002 \pm 0.004$ & $0.01 \pm 0.03$ \\
6 & II & $0^{*}$ & $0^{*}$ & $0^{*}$ \\
9 & II & $0^{*}$ & $0^{*}$ & $0^{*}$ \\
1 & III & $0.003 \pm 0.003$ & $0.003 \pm 0.002$ & $0.011 \pm 0.009$ \\
2 & IV & $0.016 \pm 0.004$ & $0.011 \pm 0.003$ & $0.041 \pm 0.010$ \\
3 & IV & $0.011 \pm 0.004$ & $0.011 \pm 0.004$ & $0.10 \pm 0.03$ \\
5 & IV & $0.032 \pm 0.011$ & $0.023 \pm 0.007$ & $0.12 \pm 0.04$ \\
7 & IV & $0.007 \pm 0.003$ & $0.007 \pm 0.004$ & $0.033 \pm 0.017$ \\
8 & IV & $0.007 \pm 0.004$ & $0.010 \pm 0.005$ & $0.06 \pm 0.03$ \\
10 & IV & $0.011 \pm 0.004$ & $0.022 \pm 0.007$ & $0.10 \pm 0.03$ \\
\hline
\end{tabular}


Table 6.4: $v_{e}$ values obtained from tracer kinetic modelling using the NordicIce software package where the AIF used in the analysis was $\mathrm{AIF}_{\phi}, \mathrm{VOF}_{\phi}$ (time shifted) or $\mathrm{VOF}_{|S|}$ (time shifted). Note: entries marked $0^{*}$ indicate that NordicIce was not able to perform the fitting due to insufficient tumour enhancement.

\begin{tabular}{|ccccc|}
\hline \multicolumn{5}{|c|}{$v_{e}$} \\
\hline No. & Grade & $\mathrm{AIF}_{\phi}$ & $\mathrm{VOF}_{\phi}$ & $\mathrm{VOF}_{|S|}$ \\
\hline 4 & II & $0.007 \pm 0.009$ & $0.1 \pm 0.3$ & $0.07 \pm 0.11$ \\
6 & II & $0^{*}$ & $0^{*}$ & $0^{*}$ \\
9 & II & $0^{*}$ & $0^{*}$ & $0^{*}$ \\
1 & III & $0.009 \pm 0.008$ & $0.006 \pm 0.004$ & $0.008 \pm 0.007$ \\
2 & IV & $0.10 \pm 0.07$ & $0.065 \pm 0.019$ & $0.10 \pm 0.010$ \\
3 & IV & $0.16 \pm 0.08$ & $0.14 \pm 0.05$ & $0.6 \pm 0.3$ \\
5 & IV & $0.08 \pm 0.06$ & $0.13 \pm 0.11$ & $0.12 \pm 0.09$ \\
7 & IV & $0.097 \pm 0.016$ & $0.085 \pm 0.017$ & $0.39 \pm 0.08$ \\
8 & IV & $0.3 \pm 0.2$ & $0.23 \pm 0.11$ & $0.47 \pm 0.21$ \\
10 & IV & $0.100 \pm 0.018$ & $0.11 \pm 0.04$ & $0.37 \pm 0.22$ \\
\hline
\end{tabular}

Table 6.5: Linear fit between perfusion parameters derived using the indicated inputs. Eight studies were included in the comparison.

\begin{tabular}{|cccc|}
\hline Parameter & Model input & Linear Fit & $\mathrm{R}$ \\
\hline$K^{\text {trans }}$ & $\mathrm{AIF}_{\phi}$ vs $^{\text {VOF }}$ & $\mathrm{y}=(0.77 \pm 0.09) \mathrm{x}+(0.01 \pm 0.009)$ & $\mathrm{R}=0.96$ \\
$K^{\text {trans }}$ & $\mathrm{VOF}_{|S|}$ vs VOF $_{\phi}$ & $\mathrm{y}=(3.5 \pm 0.4) \mathrm{x}+(0.022 \pm 0.008)$ & $\mathrm{R}=0.96$ \\
$v_{e}$ & $\mathrm{AIF}_{\phi}{\text { vs } \mathrm{VOF}_{\phi}}$ & $\mathrm{y}=(1.4 \pm 0.3) \mathrm{x}+(-0.04 \pm 0.04)$ & $\mathrm{R}=0.86$ \\
$v_{e}$ & $\mathrm{VOF}_{|S|}$ vs VOF $_{\phi}$ & $\mathrm{y}=(2.3 \pm 1.0) \mathrm{x}+(0.00 \pm 0.12)$ & $\mathrm{R}=0.67$ \\
$v_{p}$ & $\mathrm{AIF}_{\phi}{\text { vs } \mathrm{VOF}_{\phi}} \mathrm{y}=(1.0 \pm 0.3) \mathrm{x}+(0.000 \pm 0.004)$ & $\mathrm{R}=0.81$ \\
$v_{p}$ & $\mathrm{VOF}_{|S|}$ vs VOF $_{\phi}$ & $\mathrm{y}=(4.5 \pm 0.8) \mathrm{x}+(0.006 \pm 0.008)$ & $\mathrm{R}=0.92$ \\
\hline
\end{tabular}




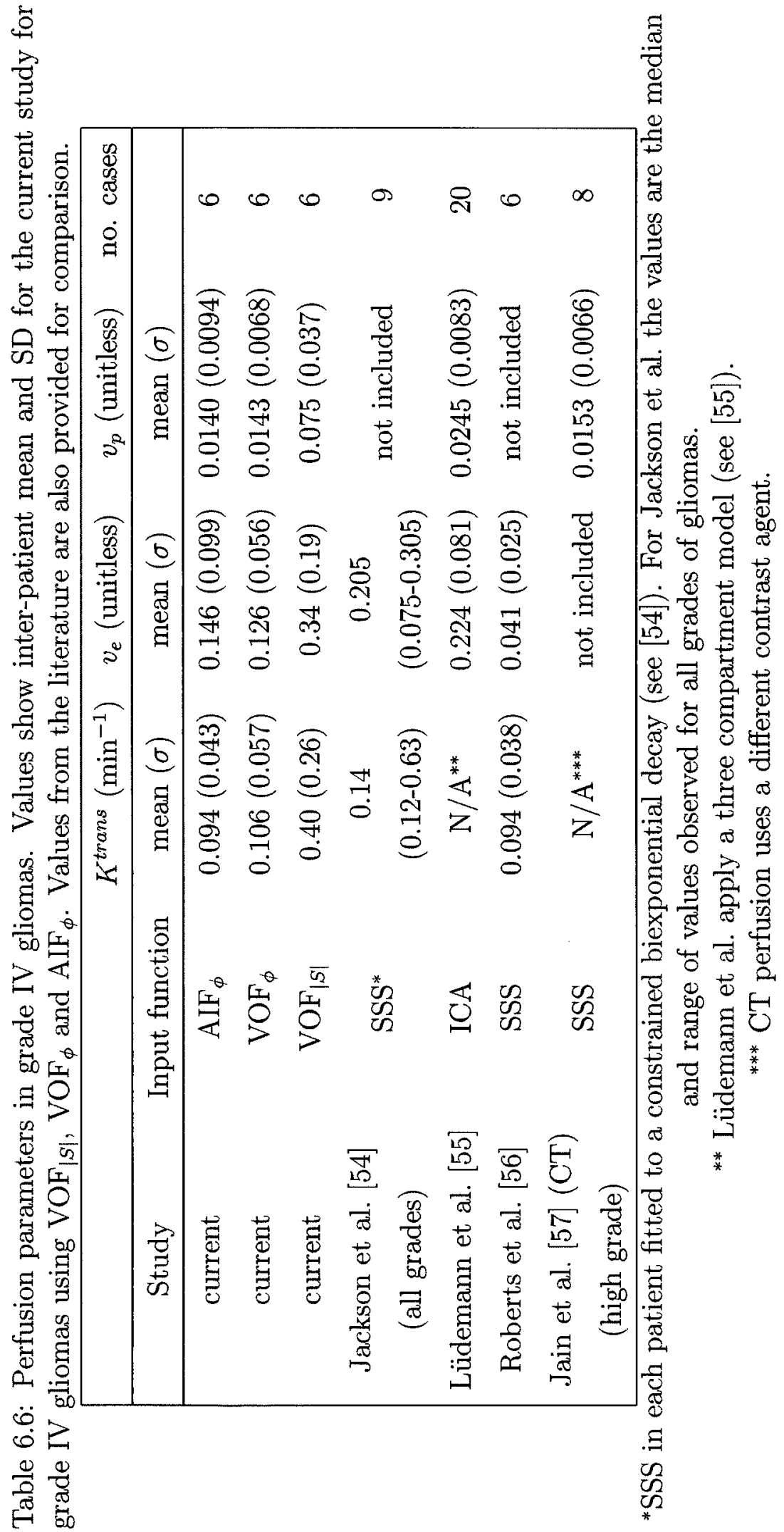




\subsection{Discussion}

DCE-MRI has the potential to become a very important method for determining tissue perfusion parameters and it may have numerous clinical applications, one of the most important being in tumour assessment. However, obtaining accurate perfusion parameters with DCE-MRI has proven difficult because it is hard to obtain a reliable arterial input function; good knowledge of the AIF is vital if accurate perfusion parameter values are to be derived using tracer kinetic modelling of the perfusion data. The main goal of this thesis is to demonstrate that a much more accurate AIF can be obtained from the MR data set if the phase of the MR signal is used rather than the more conventional approach which uses signal magnitude.

In this chapter, two important advancements in this area are demonstrated in a study of brain tumour patients. These advancements are:

1) The $\phi$-derived AIF is shown to be a useful approximation of the true AIF; it is in good agreement with the population AIF given by Parker et al. [38] for the group of patients studies here. In contrast, it was not possible, with the protocol used here, to obtained a $|S|$-derived AIF.

2) $\mathrm{The}_{\mathrm{AIF}}$ and $\mathrm{VOF}_{\phi}$ were found to be very similar in shape for these patients. Furthermore, after compensation for the time lag of the $\mathrm{VOF}_{\phi}$ relative to the $\mathrm{AIF}_{\phi}$, the $\mathrm{VOF}_{\phi}$, which is often much easier to measure, was successfully used in the tracer kinetic modelling as a good approximation to the AIF.

A 2D acquisition protocol was chosen for the brain tumour cohort studied here since it provides better time resolution that the corresponding $3 \mathrm{D}$ protocol. This is important for brain studies because of the low permeability to the CA of the BBB in healthy brain. With this protocol it was possible to set up two separate slice groups. One group (4 slices) was situated at the location of the tumour and the other group (1 slice) was placed at the level of the neck to measure the AIF. This arrangement 
worked well for the measurement of the $\mathrm{AIF}_{\phi}$ but it was not possible to measure $\mathrm{AIF}_{|S|}$ for this protocol. This is primarily because of in-flow effects (described in Chapter 3) related to the fast moving blood in these arteries. The $[\mathrm{CA}]$ was also high enough that the assumption of a one-to-one relationship between the $|S|$ and $[\mathrm{CA}]$ was not reliable in the arteries ( $T_{2}^{*}$ effects). Additionally, the pre-injection $T_{1}$ value of the blood in the arteries cannot be measured, again because of in-flow effects, and thus must be estimated from values reported in the literature. None of these effects is a significant problem when $\phi$ is used instead.

Since it was not possible to measure $\mathrm{AIF}_{|S|}$ with the protocol used it was clearly also not possible to do a direct comparison of $\mathrm{AIF}_{|S|}$ and $\mathrm{AIF}_{\phi}$ beyond stating that it was possible to measure $\mathrm{AIF}_{\phi}$ but not $\mathrm{AIF}_{|S|}$. Instead, the validity of $\mathrm{AIF}_{\phi}$ had to be established by comparing it to $\mathrm{AIF}_{P}$, the population averaged AIF reported in the literature by Parker et al. [38]. $\mathrm{AIF}_{P}$ was generated from measurements in either the descending aorta or iliac arteries. This AIF was measured by MRI and is believed to be a good AIF because they used a large vessel volume and measured the AIF in the center of the volume, they ensured that the $B_{1}$ field was uniform at the location of the AIF measurement and then only used the top 5\% of enhancing pixels. In addition, they used a short echo time, $T_{E}=0.82 \mathrm{~ms}$ to avoid $T_{2}^{*}$ effects. This population AIF provides a useful comparison because they imaged 23 patients 5 times so it provides a good sense of the variablility expected. The $\mathrm{SD}$ for $\operatorname{AIF}_{P}$ at the peak was $\pm 3 \mathrm{mM}$ and the SD in the washout region was approximately $\pm 0.3 \mathrm{mM}$.

As can be seen from Table 6.1 our $\mathrm{AIF}_{\phi}$ compared very well with $\mathrm{AIF}_{P}$. Although the peak value of $\mathrm{AIF}_{\phi}$ was a bit higher than the peak value of $\mathrm{AIF}_{P}$, it was still within one SD. The same is true for the area under the peak. The washout part of the two functions were in very good agreement. When considering the accuracy of the input and output functions, it is important to consider that some patient-to-patient variability is expected and that the standard deviations presented in Table 6.1 are a 
combination of uncertainty and true patient-to-patient variability.

Although we were successful in measuring good $\phi$-derived AIFs in the neck, it would be preferable to measure the AIF from tumour containing images so that 1) the acquisition of the extra slice can be avoided and 2) the input can be determined closer to the tumour. In principle, the AIF can be determined from arteries in these slices but the orientation and size of these arteries often make this difficult.

For brain perfusion studies with healthy subjects, it is known that the AIF and the VOF will be very similar; the VOF will be delayed and slightly broader relative to the AIF but the differences will not be large. This is a consequence of the MRI $\mathrm{CA}$ not being able to pass through a healthy BBB. Thus, all the CA entering the brain, which determines the AIF, will also leave the brain, determining the VOF. For brain tumour patients, some of the CA will leak into the brain tumour where the BBB is compromised but, in most cases this will only be a small fraction of the CA entering the brain and it is expected to have only a minor effect on the VOF. So, even for brain tumour patients, the assumption that the AIF and VOF are very similar was felt to be quite reasonable. This assumption was tested by comparing AIFs and VOFs measured in our small cohort of brain cancer patients.

For all of the images through the brain tumour acquired as part of this study, the SSS was large and easily identified. It was therefore chosen as the location from which to determine both $\mathrm{VOF}_{\phi}$ and $\mathrm{VOF}_{|S|}$. The $\mathrm{VOF}_{|S|}$ s were quite variable, they did not compare well with $\mathrm{AIF}_{P}$ and were in general found to be quite unreliable. On the other hand, the $\mathrm{VOF}_{\phi} \mathrm{s}$ compared very well with both $\mathrm{AIF}_{\phi}$ and $\mathrm{AIF}_{P}$. There was a clear difference in the VOFs obtained using $|S|$ versus $\phi$ for this protocol, which had a significant impact on the perfusion parameters. This behaviour likely reflects the insensitivity of $\phi$ to blood flow compared to $|S|$ (see also Chapter 3). This is particularly true in a $2 \mathrm{D}$ exam as seen in the slice to slice variability of the $\mathrm{VOF}_{|S|}$. 
The small differences and similarities observed in the $\mathrm{AIF}_{\phi}$ and $\mathrm{VOF}_{\phi}$ were consistent with expectations. The $\mathrm{VOF}_{\phi}$ was shifted in time, was more spread out (disperse) and the peak height was reduced as compared to the $\mathrm{AIF}_{\phi}$. These results are consistent with a range of CA transit times which is expected for a complex vasculature (see Section 2.2.2). The $\mathrm{AIF}_{\phi}$ and $\mathrm{VOF}_{\phi}$ had, statistically speaking, the same $\mathrm{AUC}_{\text {peak }}$ and washout height, indicating that there was minimal loss of CA through leakage across the BBB. These results provide self consistency for the $\phi$ method as potential errors due to partial volumes and signal leakage would not likely affect AIF $_{\phi}$ and $\mathrm{VOF}_{\phi}$ in the same way and would likely result in inconsistent results (see Section 2.8.3). The similarity in $\mathrm{AUC}_{\text {peak }}$ and washout height supports the hypothesis that a time shifted $\mathrm{VOF}_{\phi}$ could be used in place of the $\mathrm{AIF}_{\phi}$. This was further supported by the perfusion map analysis which showed excellent correlation between the values obtained using both methods. This suggests that for the tracer kinetic model applied to this study the small differences in peak height and dispersion between the AIF $_{\phi}$ and $\mathrm{VOF}_{\phi}$ do not significantly influence the measured perfusion parameters.

This study also explored some of the concerns raised in the phantom and geometry factor chapters, in an in vivo model. The impact of the orientation and shape of the vessel, the diameter of the vessel relative to the spatial resolution of the image and the echo time of the input and output functions were investigated.

The orientation and shape of the SSS contributed to the success of the phase technique proposed in this thesis. For a patient lying supine in the MRI scanner, the part of the vessel which runs along the back of the head approximates a long, straight tube, parallel with the main magnetic field. Although the vessel does have a small amount of curvature (radius of curvature $\approx 40 \mathrm{~mm}$ ), the phantom experiments and simulations show that for this amount of curvature a straight vessel approximation can be applied (see Chapter 4 and Chapter 5). The arteries in the neck slice were in general, very straight and thus curvature was not an issue. 
The success of the technique is also in part owing to the relatively large width of the SSS and the carotid arteries relative to the spatial resolution, resulting in small signal leakage and partial volume effects, which improves accuracy. The diameter of the SSS in adults is typically $4-7 \mathrm{~mm}$, based upon a review of 28 Computed Tomography (CT) studies performed at our institution (in reasonable agreement with the literature) [58], whereas the in-plane resolution was $1.8 \mathrm{~mm}$. There was however, a significant difference found between the larger and smaller vessels located in the neck slice. This difference gives an estimate of the effect of signal leakage in an in vivo case. Despite this difference the $[\mathrm{CA}]$ determined using the $\phi$ measured in these smaller vessels is still more accurate than the $[\mathrm{CA}]$ derived from $|S|$.

The echo time used in the acquisition of the dynamic series affects the quality of the results since the phase accumulation of the signal increases with $T_{E}$. If the $T_{E}$ is too short then the $\mathrm{SNR}_{\phi}$ will be very small but if $T_{E}$ is too long, phase wrap and low $\mathrm{SNR}_{|S|}$ can cause problems and the time resolution is increased. In this study, the short $T_{E}$ of $2.06 \mathrm{~ms}$ gave very similar results to those obtained with the longer $T_{E}$ of $5.48 \mathrm{~ms}$. There was an increase in the SD in the washout for the short $T_{E}$, although the mean values were the same, as well as the peak height and $\mathrm{AUC}_{\text {peak }}$. This is a better result for the short $T_{E}$ than observed in the phantom study presented in Chapter 4, where the results indicated a loss in accuracy at a shorter $T_{E}$. The similarity in the results reported here for the two $T_{E}$ 's also suggests that there is not a large extravascular contribution of the signal since, if there were, it would likely have a different effect at each $T_{E}$. Furthermore, in vivo the signal phase will normally be insensitive to such contaminating signals since the magnetic susceptibility of the surrounding tissue will usually be similar to that inside the vessel. The successful measurement of the VOF with the shorter $T_{E}$ also shows that this rather short $T_{E}$ could be used if needed. This is the case in the next chapter where a 3D acquisition is used. 
Although, in general, the results were the same for both $T_{E}$ 's, there were cases were the $\mathrm{VOF}_{\phi}$ measured using the shorter $T_{E}$ were rejected for some slices through the SSS because the $[\mathrm{CA}]$ measured were not reasonable or the shape of the VOF was unrealistic. In two studies the $\mathrm{VOF}_{\phi}$ from all the slices were unphysical for the short $T_{E}$ but acceptable for the long $T_{E}$, thus a VOF was obtained for the longer $T_{E}$ but not for the shorter $T_{E}$. One possible way to improve the results obtained with a shorter $T_{E}$ is to apply more sophisticated $\mathrm{VOF}_{\phi}$ selection scheme. Once such input selection scheme is explored for a $2.56 \mathrm{~ms} T_{E}$ DCE-MRI protocol in the proceeding chapter, Chapter 7.

Ultimately, the most important question to consider with this study is whether or not the proposed method will give correct values for the perfusion parameters. Unfortunately, it was not possible in this study to measure the perfusion parameters for each subject using a more established technique such as PET or CT as well as MRI. However, our results $v_{p}$ are consistent (within $1 \mathrm{SD}$ ) with literature values obtained using CT techniques for similar groups of subjects (see Table 6.6). CT perfusion studies use a different contrast agent so comparison of $K^{\text {trans }}$ is not possible for these studies. The $K^{\text {trans }}$ values measured using $\mathrm{AIF}_{\phi}$ and $\mathrm{VOF}_{\phi}$ were consistent with other MRI literature values (within 1 SD) (see Table 6.6). The value for $v_{e}$ measured using $\mathrm{AIF}_{\phi}$ and $\mathrm{VOF}_{\phi}$ fell between reported values. The values measured using $\mathrm{VOF}_{|S|}$ were inconsistent in all cases.

\subsection{Conclusions}

In conclusion, this study demonstrated that perfusion can be successfully measured using signal phase. For the case of $2 \mathrm{D}$ DCE-MRI, the $\mathrm{VOF}_{\phi}$ s were significantly better than the $\mathrm{VOF}_{|S|}$ s. The $\mathrm{AIF}_{\phi}$ and $\mathrm{VOF}_{\phi}$ had a more reasonable height, $\mathrm{AUC}_{\text {peak }}$ and showed less variability. The perfusion parameters derived from $\mathrm{VOF}_{\phi}$ were more in 
line with other published work. The validation of a 2D DCE-MRI protocol allows for improved time resolution as compared to a 3D acquisition. In addition, it was shown that, although there is some difference between the perfusion parameters calculated using the $\mathrm{VOF}_{\phi}$, as opposed to the $\mathrm{AIF}_{\phi}$, the former can likely be used in place of the latter when it is more convenient (i.e. a vein is present in the tumour image block). This study also showed that even a $T_{E}$ of $2.06 \mathrm{~ms}$ can produce accurate VOFs, although the longer $T_{E}$ of $5.48 \mathrm{~ms}$ was more reliable. This suggests a shorter $T_{E}$ can be used under the appropriate conditions. 


\section{Chapter 7}

\section{Prostate Tumour Study}

\subsection{Introduction}

Dynamic contrast-enhanced MRI (DCE-MRI) has shown promise as an important diagnostic tool for extracranial tumours and contrast enhanced imaging is often included in an MRI exam of tumours. Despite its prevalence, a standardized technique for measuring perfusion parameters from DCE-MRI exams has not been agreed upon. Variations in the exam protocol lead to variations in the final perfusion values making it difficult, if not impossible to compare results between institutions or to conduct multi-institutional studies. This lack of consensus is largely due to the difficulty in obtaining an accurate and reproducible arterial input function (AIF). Population averaged AIFs have been proposed for this purpose but it is preferable to measure the individual AIF for each patient, since variation between patients is expected.

In the previous chapter, a method for incorporating AIFs derived from $\phi$, the phase of the MR signal, into MRI perfusion measurements for intra-cranial tumours was successfully developed. This study demonstrated that the $\phi$-derived AIFs, AIF $_{\phi}$, were more consistent with a population AIF from the literature, [38] than the $|S|$ derived AIFs, AIF $|S|$. This study also showed that these differences in AIF resulted in dramatically different perfusion parameter values. This reinforces the need for 
a standardized method of measuring the AIF which does not vary with DCE-MRI protocol. There are several reasons to suggest that $\mathrm{AIF}_{\phi}$ measurements are more reliable that $\mathrm{AIF}_{|S|}$ measurements: 1) $\phi$ does not saturate at high contrast agent concentrations, [CA], 2) $\phi$ is not affected by RF inhomogeneities 3) $\phi$ is not affected by blood flow and 4) the conversion to [CA] from $\phi$ does not require knowledge of the pre-contrast injection $T_{1}, T_{1,0}$.

To expand upon the success of the intra-cranial study presented in Chapter 6, a study of extra-cranial tumours is warranted because there are several additional challenges when imaging extra-cranial tumours. These challenges include a lower signal-to-noise ratio (SNR), since the body coils are used instead of the head coil, and there is potentially more motion, particularly if the tumour location is affected by respiratory motion. Thus, the logical next step is to explore the application of the technique developed in Chapter 6 to extra-cranial tumours. The specific goals of this chapter are to establish a dynamic contrast enhanced-MRI (DCE-MRI) protocol for perfusion studies of prostate tumours that incorporates $\phi$ and to compare the results computed with this protocol to those obtained via more conventional methods. The case of prostate tumours is ideal for the application of the $\phi$ technique because the imaging volume contains a large artery (iliac or femoral) which is relatively straight and parallel to $\mathbf{B}_{\mathbf{0}}$. Additionally, the imaging volume contains muscle which can be used as a reference since it is expected to have less inter-patient variability. Although work presented in this chapter focuses specifically on prostate tumours it should be applicable to other pelvic and abdominal regions as well. The extension of the technique to other tumour locations is also likely feasible provided a suitable AIF can be identified in the imaging volume.

This study was done using a 3D acquisition instead of the $2 \mathrm{D}$ acquisition used in Chapter 6. The 3D acquisition is advantageous for conventional DCE-MRI methods (i.e. using $A I F|S|$ ) because the effect of blood flowing into the volume of interest on 
signal enhancement is reduced [35]. Even so, it is still difficult to choose the correct location for measuring $\mathrm{AIF}_{|S|}$ since the magnitude signal is not uniform throughout the imaging volume. As $\phi$ is not expected to vary throughout the volume this suggests that using $\phi$ to measure the AIF may provide an AIF measurement technique which is more reproducible when different imaging protocols are used.

The application of a $3 \mathrm{D}$ acquisition also allows further exploration the $\phi$ technique at short echo times, $T_{E}$. In order to keep the time resolution reasonable, this larger $3 \mathrm{D}$ volume requires a shorter $T_{E}$ which reduces the phase $\mathrm{SNR}, \mathrm{SNR}_{\phi}$, since $\phi$ evolves over a shorter time period. However, the investigation presented in Chapter 6 showed that this shorter $T_{E}$ still has the potential to provide accurate AIF and perfusion parameter measurements.

The chapter begins by outlining, in Section 7.2, the specific DCE-MRI methods employed for this study. A more general description of the DCE method has already been given in Section 2.8. The results of this ongoing study are presented in Section 7.3. First the $\mathrm{AIF}_{\phi}$ and $\mathrm{AIF}_{|S|}$, as a function of slice position, are compared. In addition to the computation of the prostate perfusion maps, a region of interest (ROI) analysis of the obturator internus muscle (OIM) is performed as an assumed standard to compare the $K^{\text {trans }}$ and $v_{e}$ values with those found in the literature. The results of this study are then discussed in Section 7.4 and conclusions are presented in Section 7.5 .

\subsection{Methods}

\subsubsection{The clinical protocol}

The data presented in this chapter were acquired as part of a study conducted at the General Campus of the Ottawa Hospital, Ottawa, Canada. It involved twenty-seven 
prostate cancer patients undergoing routine clinical scans, which included contrast injection. All imaging was performed on a 1.5 T Siemens Symphony MR scanner using a whole-body transmit coil with flex, and spine receive coils (Siemens Medical Solutions, Erlangen, Germany). DCE-MRI was performed using a 3D spoiled gradient echo (SPGR) sequence with $T_{R}=5.8 \mathrm{~ms}, T_{E}=2.56 \mathrm{~ms}$ and $\alpha=35^{\circ}$. The matrix size was $256 \times 224 \times 20$ with a slice thickness of $5 \mathrm{~mm}$ and field of view (FOV) of $250 \times 220 \mathrm{~mm}^{2}$, leading to an in-plane resolution of $0.98 \times 0.98 \mathrm{~mm}^{2}$. The time resolution was $13.5 \mathrm{~s}$ for a total scan time of $405 \mathrm{~s}$. The Gd-based contrast agent (CA), Gadovist ( $($ Bayer Healthcare Canada) was administered intravenously using a power injector at a flow rate of $4 \mathrm{ml} / \mathrm{s}$, with saline flush. The dose used for this study was $0.2 \mathrm{mmol} \mathrm{Gd} / \mathrm{kg}$ of body weight. Four baseline time points were acquired preinjection. The power injector is controlled remotely so it was not necessary to remove the patient from the magnet for the injection. A schematic showing the approximate slice locations and anatomy of interest is shown in Fig. 7.1.

A pre-contrast $T_{1}$ map of the volume was also taken using the variable flip angle method described in Section 2.8.4. A 3D SPGR sequence with parameters $T_{R}=50$ $\mathrm{ms}, T_{E}=2.56 \mathrm{~ms}$ and $\alpha=10^{\circ}, 20^{\circ}, 40^{\circ}$ was used. The matrix size was $(256 \mathrm{x} 256 \mathrm{x}$ 20) with the same in plane resolution and slice thickness as the dynamic series.

Image analysis was performed offline using custom software written in IDL (BITT Visual Information Solutions, Boulder, CO, USA) to reconstruct the raw data and manipulate images. All statistical analysis was done using Microsoft Office Excel 2003 (®)Microsoft Corp.) or by hand unless otherwise stated.

\subsubsection{The AIF Selection Method}

Four different AIFs were compared in this study: a magnitude-derived input function, $\mathrm{AIF}_{|S|}$, a phase-derived input function, $\mathrm{AIF}_{\phi}$, and two population AIFs from the literature. To measure $\mathrm{AIF}_{|S|}$ and $\mathrm{AIF}_{\phi}$, circular ROIs were drawn in the lumen of 


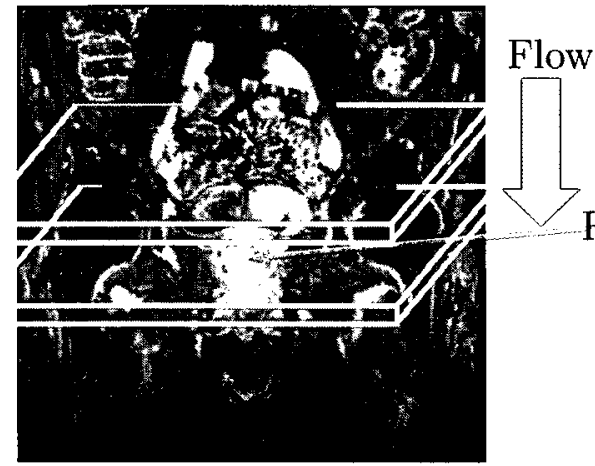

a)

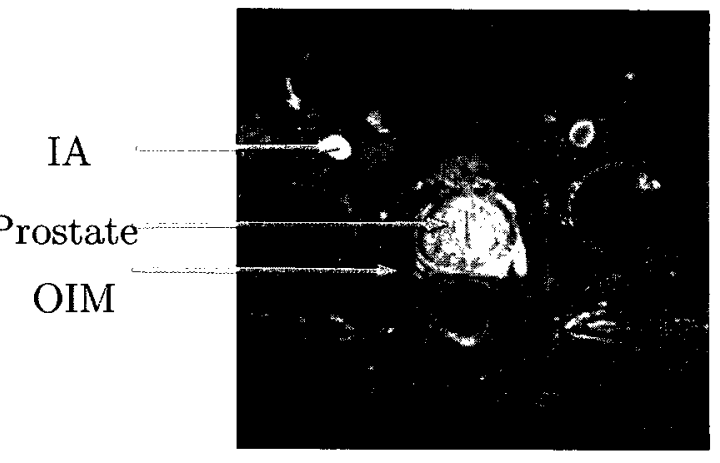

b)

Figure 7.1: Schematic of the 3D DCE-MRI protocol applied to the prostate tumour study. a) A coronal view of the male pelvis shows the approximate slice location in the exam. Note that the blood flow through the artery is in the superior to inferior direction. b) A typical image slice through the prostate, showing the iliac artery (IA) and the obturator internus muscle (OIM).

a major artery (external iliac or femoral) present in each of the twenty slices. The slice-by-slice AIFs were averaged together to form averaged AIFs with higher SNR for blocks of five contiguous slices. The slice blocks were overlapped to form a total of seven slice blocks, see Table 7.1. AIFs for these slice blocks were calculated instead of computing one average over all slices so that the variation of the AIF with slice position could be studied. The AIFs calculated from each slice block will be referred

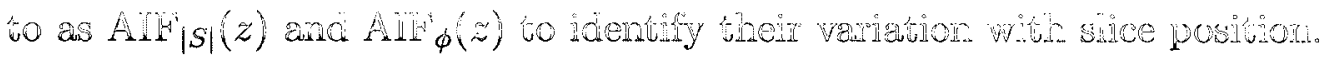

To calculate $\operatorname{AIF}_{|S|}(z)$, all pixel values within the ROI for all slices in the slice block were averaged together and [CA] was calculated from the mean $|S|$ using Eq. 2.126 from Section 2.8.5, assuming a $T_{1,0}$ of blood of $1250 \mathrm{~ms}[35] . \operatorname{AIF}_{\phi}(z)$ was calculated in a similar manner, except that, because of the low $\mathrm{SNR}_{\phi}$, it was necessary to develop a voxel acceptance scheme. Voxels within a given ROI were rejected if the cross-correlation between $|S|(t)$ and $\Delta \phi(t)$, measured as the Pearson's correlation coefficient [44], was less than 0.1. Voxels were also rejected if the $\Delta \phi(t)$ curve exhibited a positive, rather than the expected negative, slope during the last $270 \mathrm{~s}$ of the scan. The threshold of 0.1 was determined empirically by testing a range of cutoffs. On 
Table 7.1: A description of the slice grouping. The slices included in each slice block and the location of the center of the block relative to the inferior edge of the complete $3 \mathrm{D}$ slice block are given.

\begin{tabular}{|ccc|}
\hline Slice Block & Slices Included & Center of Block $(\mathrm{mm})$ \\
\hline 1 & $1-5$ & 12.5 \\
2 & $3-7$ & 22.5 \\
3 & $6-10$ & 37.5 \\
4 & $8-12$ & 47.5 \\
5 & $11-15$ & 62.5 \\
6 & $13-17$ & 72.5 \\
7 & $16-20$ & 87.5 \\
\hline
\end{tabular}

average, $12 \%$ of the voxels were rejected for having a cross-correlation between $|S|(t)$ and $\Delta \phi(t)$ of less than 0.1 , and $24 \%$ were rejected for having a positive slope in $\Delta \phi(t)$ during the last $270 \mathrm{~s}$. Subsequently, $\Delta \phi(t)$ was converted to [CA] using Eq. 2.114. The geometry factor was calculated assuming a straight vessel at an angle, $\theta$, of $15^{\circ}$ with respect to $\mathbf{B}_{\mathbf{0}}$ since for the group of twenty-seven subjects in this study it was determined that $\theta=(15 \pm 6)^{\circ}$.

To facilitate the validation of the perfusion modelling, two population AIFs were also used. This is not an uncommon practice in the case of body tumours, where the AIF can be difficult to measure. The first, $\mathrm{AIF}_{T K}$, is a population AIF developed by Tofts \& Kermode [16]. This AIF is in the form of a bi-exponential decay fitted to blood sampling data and has been used by a large number of researchers. The second, $\mathrm{AIF}_{P}$, is a high temporal resolution, population-averaged AIF, recently developed by Parker et al. [38]. This population AIF was measured in either the descending aorta or iliac arteries using MRI. Both population AIFs were scaled to reflect the correct CA injection dose of $0.2 \mathrm{mmol} / \mathrm{kg}$.

As with the analysis presented in Chapter 6, parameters are defined to facilitate the comparison of different AIFs. The peak height is defined as the maximum [CA] 
observed in the AIF, in mM. The washout is defined as the mean [CA] over $121.5 \mathrm{~s}$ beginning $189 \mathrm{~s}$ after the peak in the [CA] curve. This range was chosen to encompass a portion of the AIF curve where the change in [CA] was minimal and also to include a sufficient number of data points.

\subsubsection{The Obturator Internus Muscle}

It was assumed that the OIM has a macroscopically uniform structure and thus, will have uniform perfusion parameters over its entire structure. In this case, the average magnitude signal (scaled to [CA]) can be used as the tissue response function (TRF), instead of determining the TRF for each pixel separately. This improves the SNR, which reduces error in the perfusion modelling. A large square ROI of $86 \mathrm{~mm}^{2}$ was drawn on the left and right OIM for three contiguous slices identified to contain the largest cross-section of OIM. For each 3D image set, the magnitude signal of the OIM was calculated as the average signal over all six of these ROIs (left and right $\mathrm{x} 3$ slices). The magnitude signal of the OIM as a function of time, $|S|(t)$, was converted to $[\mathrm{CA}]$ in the tissue using Eq. 2.126. $T_{1,0}$ in the muscle was assumed to be $1000 \mathrm{~ms}$ [59]. Tracer kinetic modelling, using all four AIFs, was performed on the [CA]-vs-time data in the OIM to obtain $K^{\text {trans }}$ and $v_{e}$, assuming a negligible plasma compartment, see Eq. 2.31 and [60]. This analysis was also repeated for muscle $T_{1}$ values of $850 \mathrm{~ms}$ and $1150 \mathrm{~ms}$ to check the sensitivity of the computed perfusion parameters to the assumed $T_{1}$ values.

\subsubsection{The Prostate}

A map of $[\mathrm{CA}]$ time courses was calculated pixel-by-pixel with in house software written in IDL using Eq. 2.126. $T_{1,0}$ was assumed to be $1000 \mathrm{~ms}$ [59] as the measured $T_{1,0}$ map appeared to suffer from errors due to $\mathbf{B}_{\mathbf{1}}$ inhomogeneities and was determined 
to be unreliable. Once the pixel-by-pixel tissue [CA] maps and the input and output functions were calculated, they were imported into a perfusion modelling software suite for the calculation of the perfusion parameters.

Pixel-by-pixel perfusion maps of the prostate gland were generated using NordicIce $₫$ (NordicImagingLab, Bergen, Norway) for a subset of eleven studies using $\mathrm{AIF}_{|S|}$ and $\mathrm{AIF}_{\phi}$ which were evaluated as described above. The program uses the model described by Eq. 2.34 and the method of Murase [53] to extract the perfusion parameters $K^{\text {trans }}, v_{p}$ and $v_{e}$ and assumes a default haematocrit ratio of 0.45 .

In the case of highly perfused tissue, such as prostate gland and tumour, the assumption of a negligible vascular compartment may not be valid and thus a model which includes this parameter was chosen. Perfusion studies of the prostate often do not include the vascular compartment in the model, because of the low temporal resolution. Therefore, to facilitate comparison with values reported in the literature, this two parameter model, Eq. 2.31, was also applied. This modelling was performed using software written in house in IDL. In addition to the perfusion parameters the maximum $[\mathrm{CA}]$ of the whole tumour was also calculated for each study, to facilitate comparison with the literature.

Pixel-by-pixel maps of the perfusion parameters were saved and imported into Image $\AA$ (National Institute of Health, USA) for further analysis. ROIs where drawn around the whole prostate gland, including the tumour, for three central slices. The mean and SD of the perfusion parameters for each study measured using $\mathrm{AIF}_{|S|}$ and $\mathrm{AIF}_{\phi}$ were calculated and compared by linear regression using Origin 5.0@ (Microcal Software Inc, Northampton, MA, USA). 


\subsection{Results}

Results from the prostate tumour study are presented in this section. First $\mathrm{AIF}_{|S|}(z)$ and $\operatorname{AIF}_{\phi}(z)$ are compared, as a function of slice position. These AIFs are then used to calculate $K^{\text {trans }}$ and $v_{e}$ in the OIM as a function of slice position. Once the optimal $\mathrm{AIF}_{|S|}$ and $\mathrm{AIF}_{\phi}$ are established, the perfusion values found for the OIM using these optimal AIFs and the population AIFs are compared. Finally, perfusion parameter results in the prostate using the optimal $\mathrm{AIF}_{|S|}$ and $\mathrm{AIF}_{\phi}$ are compared.

\subsubsection{AIF Method Comparison}

In Fig. 7.2, $\operatorname{AIF}_{|S|}(z)$ and $\operatorname{AIF}_{\phi}(z)$, averaged over all studies, are shown as a function of slice position. Note that the full time course is not displayed. It can be seen that the average $\mathrm{AIF}_{|S|}(z)$ varies significantly as a function of slice position, first increasing then decreasing in height from the most inferior (slice block 1) to the most superior (slice block 7) location. Both the peak height and washout vary significantly with slice block position (ANOVA, $p<0.0001$ ). The $\operatorname{AIF}_{\phi}(z)$ do not vary as significantly with slice position: the peak height variation was not statistically significant (ANOVA, $p=0.21$ ) and the washout variation, although statistically significant (ANOVA, $p<0.0001)$, was less than for the $\operatorname{AIF}_{|S|}(z)$. The F-value obtained from an ANOVA of the washout was ten times less for $\operatorname{AIF}_{\phi}(z)$ than for $\operatorname{AIF}_{|S|}(z)(F=5.6$ vs $F=51)$. It can also be noted that the average peak height for $\operatorname{AIF}_{|S|}(z)$ is considerably lower than for $\operatorname{AIF}_{\phi}(z)$.

\subsubsection{Perfusion in the Obturator Internus Muscle}

The OIM, located near the prostate, provides a good test of the perfusion parameters because one single ROI can be used to compare results. By applying the positionspecific AIFs to a single TRF, the effect of the position dependence of $\mathrm{AIF}_{|S|}$ and $\mathrm{AIF}_{\phi}$ 

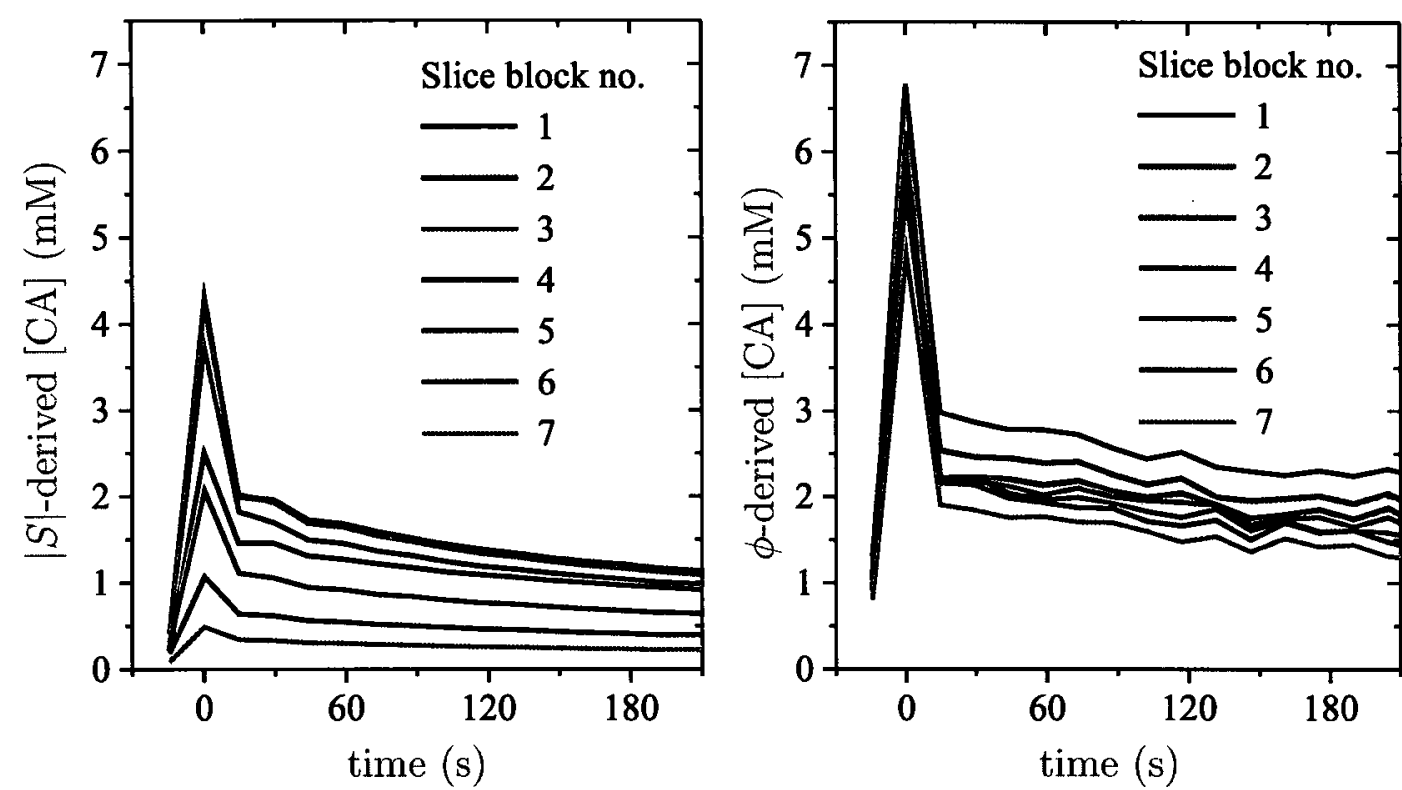

Figure 7.2: $\operatorname{AIF}_{|S|}(z)$ and $\operatorname{AIF}_{\phi}(z)$ for each slice block. Slice block 1 is most inferior and slice block 7 is most superior. Note that the full time course is not shown.

on the perfusion parameters can clearly be discerned. These results are presented in Fig. 7.3. Outliers, defined as values greater than five standard deviations from the mean value, were removed. The perfusion parameters, $K^{\text {trans }}$ and $v_{e}$, measured using $\operatorname{AIF}_{|S|}(z)$ varied significantly with slice block position with a minimum at slice block 2. It is assumed that the slice block which generates the smallest perfusion parameters provided the most accurate AIF measurements (see discussion for more details). For all further analysis using $\mathrm{AIF}_{|S|}$ only the AIF data from block 2 were used.

For the perfusion parameters calculated using $\operatorname{AIF}_{\phi}(z)$, the mean values of $K^{\text {trans }}$ did not vary significantly as a function of position (ANOVA, $p=0.50$ ). The mean value of $v_{e}$ has a small but significant variation with slice block position, however, for slice blocks more superior than slice block 2, the $v_{e}$ values obtained using $\operatorname{AIF}_{\phi}(z)$ had no statistically significant dependence on position (ANOVA, $p=0.15$ ). As a result of this generally small dependence on slice position, all further perfusion analyses using $\mathrm{AIF}_{\phi}$ were performed using an average over all slice positions. 


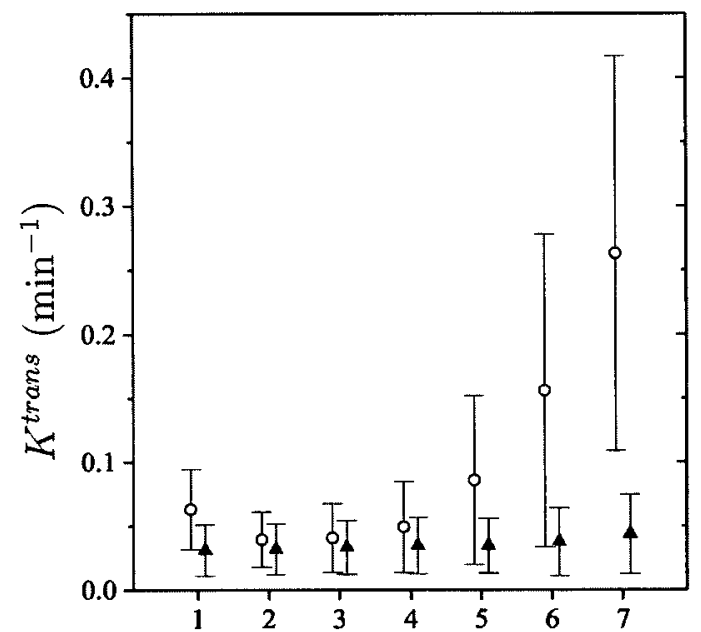

slice block number

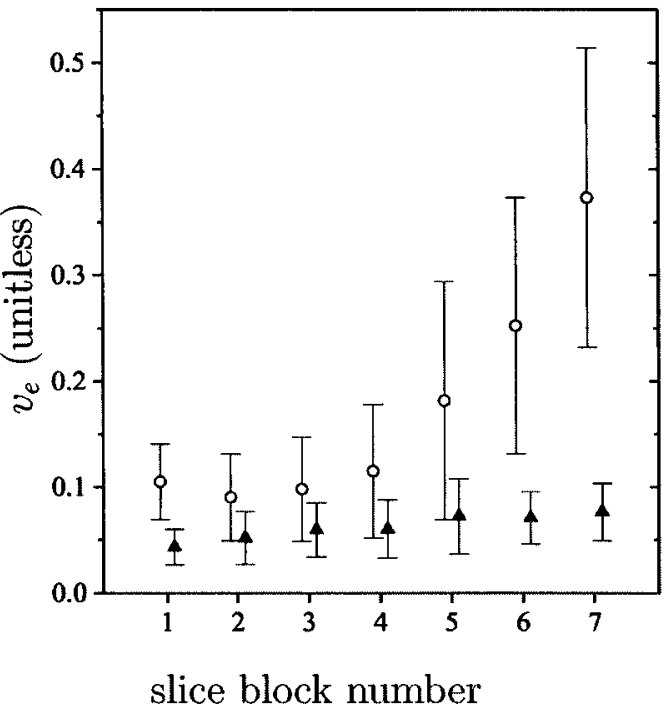

slice block number

Figure 7.3: Perfusion parameters for the OIM resulting from $\operatorname{AIF}_{|S|}(z)$ (open circles) and $\operatorname{AIF}_{\phi}(z)$ (solid triangles) as a function of slice group. Slice block 1 is the most inferior block

The optimal $\mathrm{AIF}_{|S|}$ and optimal $\mathrm{AIF}_{\phi}$ were significantly different from each other. The mean peak height was $4.2 \pm 0.3 \mathrm{mM}$ for $\mathrm{AIF}_{|S|}$ (slice block 2 only) and was $5.7 \pm 0.5$ for $\mathrm{AIF}_{\phi}$ (all blocks) which are statistically significantly different (pairwise t-test, $p=0.0004)$. The mean washout was $1.06 \pm 0.05 \mathrm{mM}$ for $\mathrm{AIF}_{|S|}$ and was $1.48 \pm 0.09 \mathrm{mM}$ for $\mathrm{AIF}_{\phi}$, which are statistically significantly different (pairwise t-test, $p<0.0001)$. Although the peak $[\mathrm{CA}]$ for $\mathrm{AIF}_{\phi}$ is higher and thus closer to the peak value of the population AIF measured by Parker et al. [38] than that of the $\mathrm{AIF}_{|S|}$, both are statistically significantly different from $\mathrm{AIF}_{P}$ peak $[\mathrm{CA}]$ (t-test, $p<0.0001$ ). The washout $[\mathrm{CA}]$ are also both statistically significantly different from the washout $[\mathrm{CA}]$ of $\mathrm{AIF}_{P}$. The optimal AIFs, averaged over all subjects, are presented in Fig. 7.4 along with $\mathrm{AIF}_{P}$ and $\mathrm{AIF}_{T K}$ for comparison.

The OIM perfusion results derived from the optimal phase and magnitude AIFs, determined above, were then compared with perfusion results obtained using the population AIFs. Figure 7.5 and Table 7.2 give a detailed side-by-side comparison 


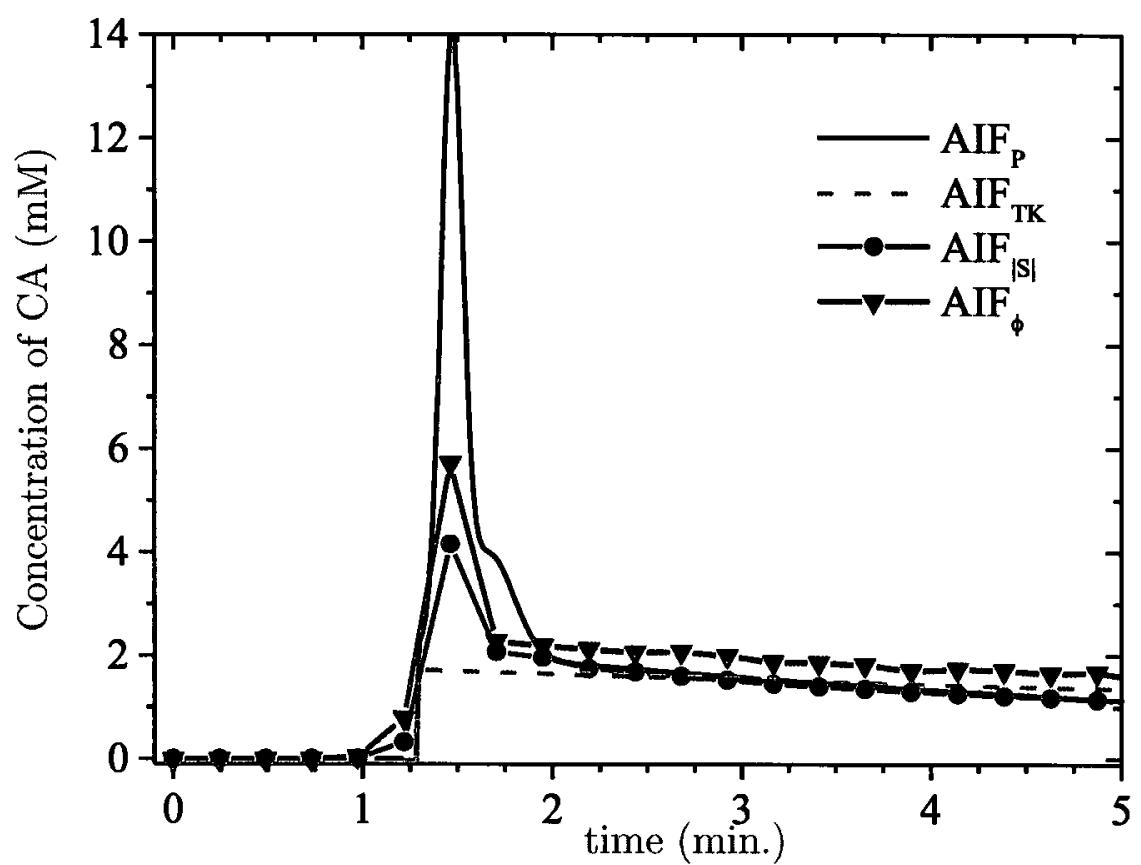

Figure 7.4: Optimal $\mathrm{AIF}_{|S|}$ and Optimal $\mathrm{AIF}_{\phi}$ averaged over 27 cases compared to two population AIF from literature. Note: the entire time course is not shown.

of the results of the perfusion modelling applied to a total ROI from the OIM using the four different $\mathrm{AIFs}\left(\mathrm{AIF}_{T K}, \mathrm{AIF}_{P}, \mathrm{AIF}_{|S|}, \mathrm{AIF}_{\phi}\right)$, as well as results from the literature.

It was determined that the $K^{\text {trans }}$ values from each method were all significantly different (pairwise t-test, $p<0.008$ ) although the distribution of values for $\operatorname{AIF}_{|S|}$, $\mathrm{AIF}_{\phi}$ as seen in Fig. 7.5 do not appear different. The order from highest to lowest was $K_{T K}^{\text {trans }}>K_{|S|}^{\text {trans }}>K_{\phi}^{\text {trans }}>K_{P}^{\text {trans }}$. For $v_{e}, \mathrm{AIF}_{P}$ and $\mathrm{AIF}_{|S|}$ gave consistent results (pairwise t-test, $p=0.77$ ), otherwise they were statistically significantly different (pairwise t-test, $p<0.001$ ). The order from highest to lowest was $v_{e, T K}>v_{e,|S|} \approx$ $v_{e, P}>v_{e, \phi}$.

Varying the $T_{1,0}$ had a small effect on the data, less than $\pm 20 \%$ for a $15 \%$ variation in the data. An overestimation of the $T_{1,0}$ generally lead to a reduction in the measured perfusion parameters and vice versa for an underestimation. 

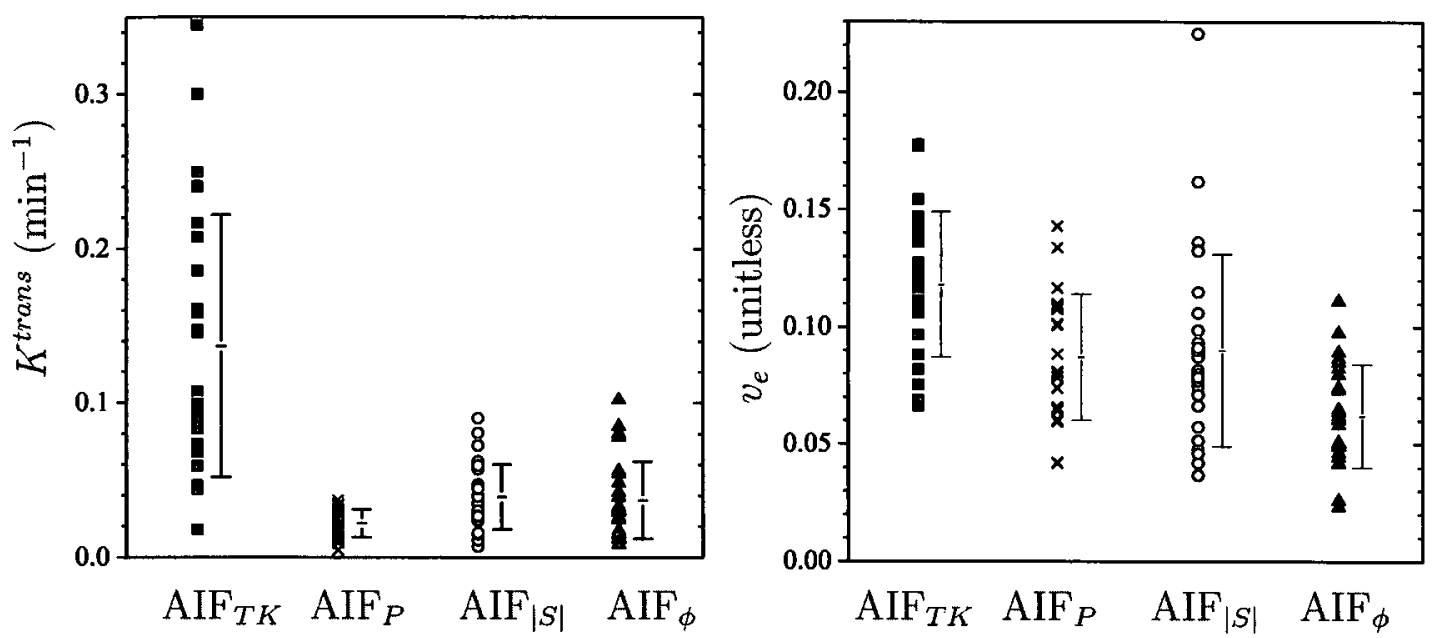

Figure 7.5: Perfusion parameter results for the OIM calculated using $\mathrm{AIF}_{|S|}$ and $\mathrm{AIF}_{\phi}$ as well as two population AIFs from the literature. Data points show values for each patient. To the right of each distribution, the inter-patient mean and SD are shown.

Table 7.2: Comparison of perfusion parameters measured in the OIM using four different AIFs, as well as results from the literature. All data is for human OIM except for the study by Yankeelov et al. which was performed in rats.

\begin{tabular}{|cccc|}
\hline & & $K^{\text {trans }}\left(\mathrm{min}^{-1}\right)$ & $v_{e}$ (unitless) \\
\hline Study & $\mathrm{AIF}$ & mean $(\sigma)$ & mean $(\sigma)$ \\
\hline current & $\mathrm{AIF}_{T K}$ & $0.137(0.085)$ & $0.118(0.031)$ \\
Padhani et al. [60] & $\mathrm{AIF}_{T K}$ & $0.156(0.095)$ & $0.113(0.021)$ \\
current & $\mathrm{AIF}_{P K}$ & $0.022(0.009)$ & $0.087(0.027)$ \\
Yankeelov et al. [61] & blood sampling & $0.021(0.002)$ & $0.067(0.010)$ \\
current & $\operatorname{AIF}_{|S|}$ & $0.039(0.021)$ & $0.090(0.041)$ \\
current & $\operatorname{AIF}_{\phi}$ & $0.037(0.025)$ & $0.062(0.022)$ \\
Buckley et al. [59] & $\mathrm{AIF}_{|S|}$ & $0.045(0.025)$ & $0.130(0.040)$ \\
\hline
\end{tabular}




\subsubsection{Perfusion in Prostate}

Pixel-by-pixel perfusion modelling was performed on eleven cases using the optimal $\mathrm{AIF}_{|S|}$ and $\mathrm{AIF}_{\phi}$ determined in the previous subsection. Typical perfusion maps for the prostate, computed using the optimal $\mathrm{AIF}_{\phi}$ are presented in Fig. 7.6 for $K^{\text {trans }}, v_{e}$ and $v_{p}$. The inter-patient mean and SD for each parameter are given in Table 7.3. No significant differences were found between the $K^{\text {trans }}$ and $v_{e}$ parameter fits calculated using optimal $\mathrm{AIF}_{|S|}$ versus optimal $\mathrm{AIF}_{\phi}$ (pairwise t-test, $p>0.77$ ). In addition, linear fits between $K_{|S|}^{\text {trans }}$ and $K_{\phi}^{\text {trans }}$ showed good correspondence: $K_{\phi}^{\text {trans }}=(0.9 \pm$ $0.2) * K_{|S|}^{\text {trans }} \pm 0.02$ with $R=0.79$ and $p=0.003$. For $v_{e}$, the correlation was not as strong, but the uncertainty in the data was also higher: $v_{e, \phi}=(0.9 \pm 0.4) * v_{e,|S|}+0.03 \pm$ 0.10 with $R=0.63$ and $p=0.04$. For $v_{p}$ there was a significant difference in the means (pairwise t-test, $p=0.004)$. The linear fit was $v_{p, \phi}=(0.52 \pm 0.14) * v_{p,|S|}+0.011 \pm 0.010$ with $R=0.77$ and $p=0.005$. These linear fits are displayed graphically in Fig. 7.7.

Results using a two parameter model which ignores $v_{p}$ were also obtained. The inter-patient mean and SD for each parameter are presented in Table 7.3. A small significant difference was found between the $K^{\text {trans }}$ values calculated using $\operatorname{AIF}_{|S|}$ and $\mathrm{AIF}_{\phi}$ (pairwise t-test, $p=0.018$ ) although no difference was seen in $v_{e}$ (pairwise t-test, $p=0.42$ ). All parameters calculated using the two parameter model were significantly different from the corresponding parameter calculated using the 3 parameter model (pairwise t-test, $p<0.004$ )

The maximum of the whole tumour mean $[\mathrm{CA}]$ for each study was calculated. The interpatient mean and SD are given in Table 7.4 for the current study along with values from the literature. These values are given in $\mathrm{mmol} / \mathrm{kg}$ per $0.1 \mathrm{mmol} / \mathrm{kg}$ initial dose of $\mathrm{CA}$ to facilitate comparison with literature values. 

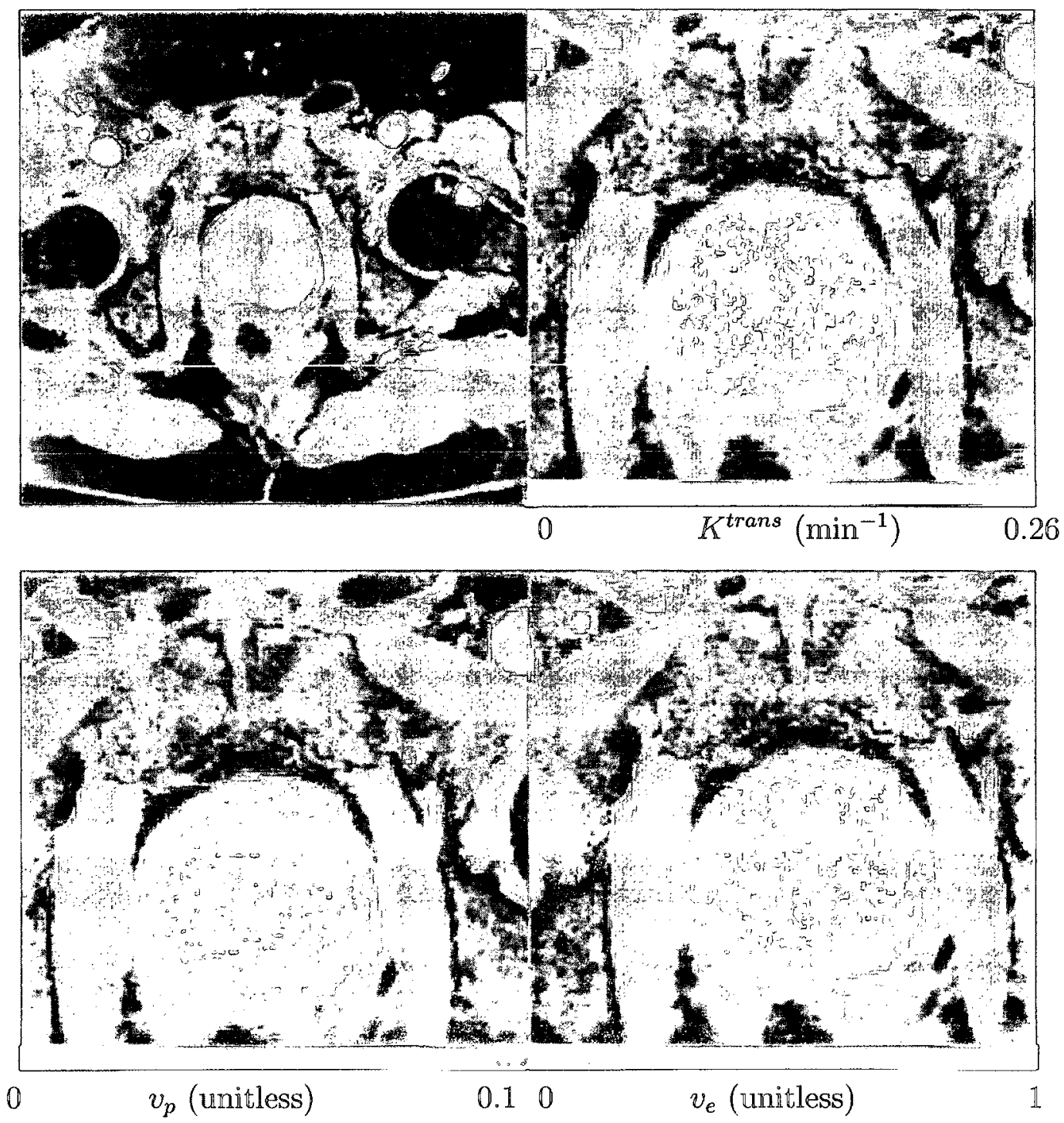

Figure 7.6: $\mathbb{K}^{\text {trans }}, v_{e}$ and $v_{p}$ computed using $\mathrm{AIF}_{\phi}$ for a typical prostate case. 


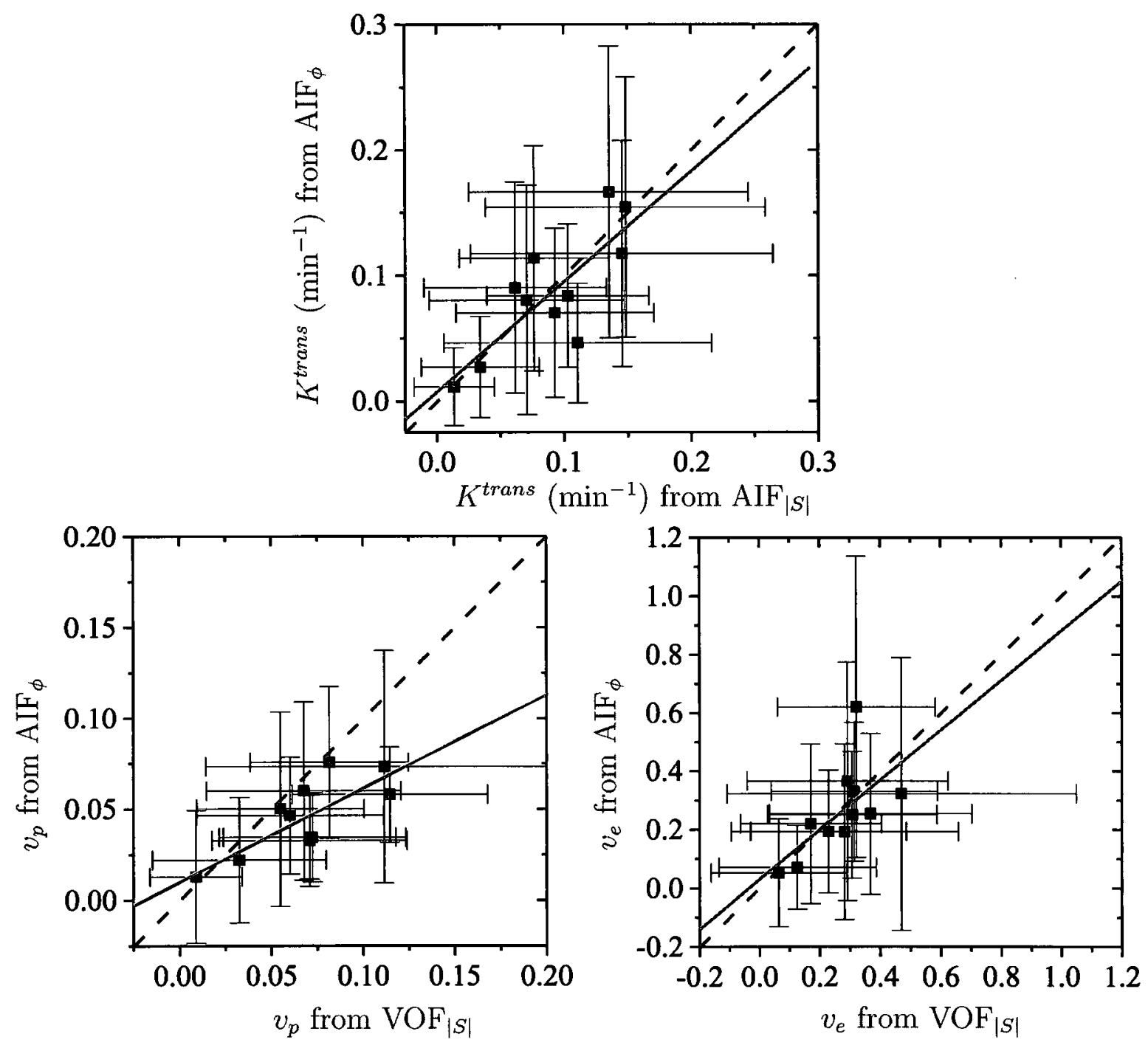

Figure 7.7: $K^{\text {trans }}$ and $v_{p}$ and $v_{e}$ measured using $\mathrm{AIF}_{|S|}$ versus $\mathrm{AIF}_{\phi}$. Error bars on the graph represent one SD in the parameter over the whole ROI for each patient. 


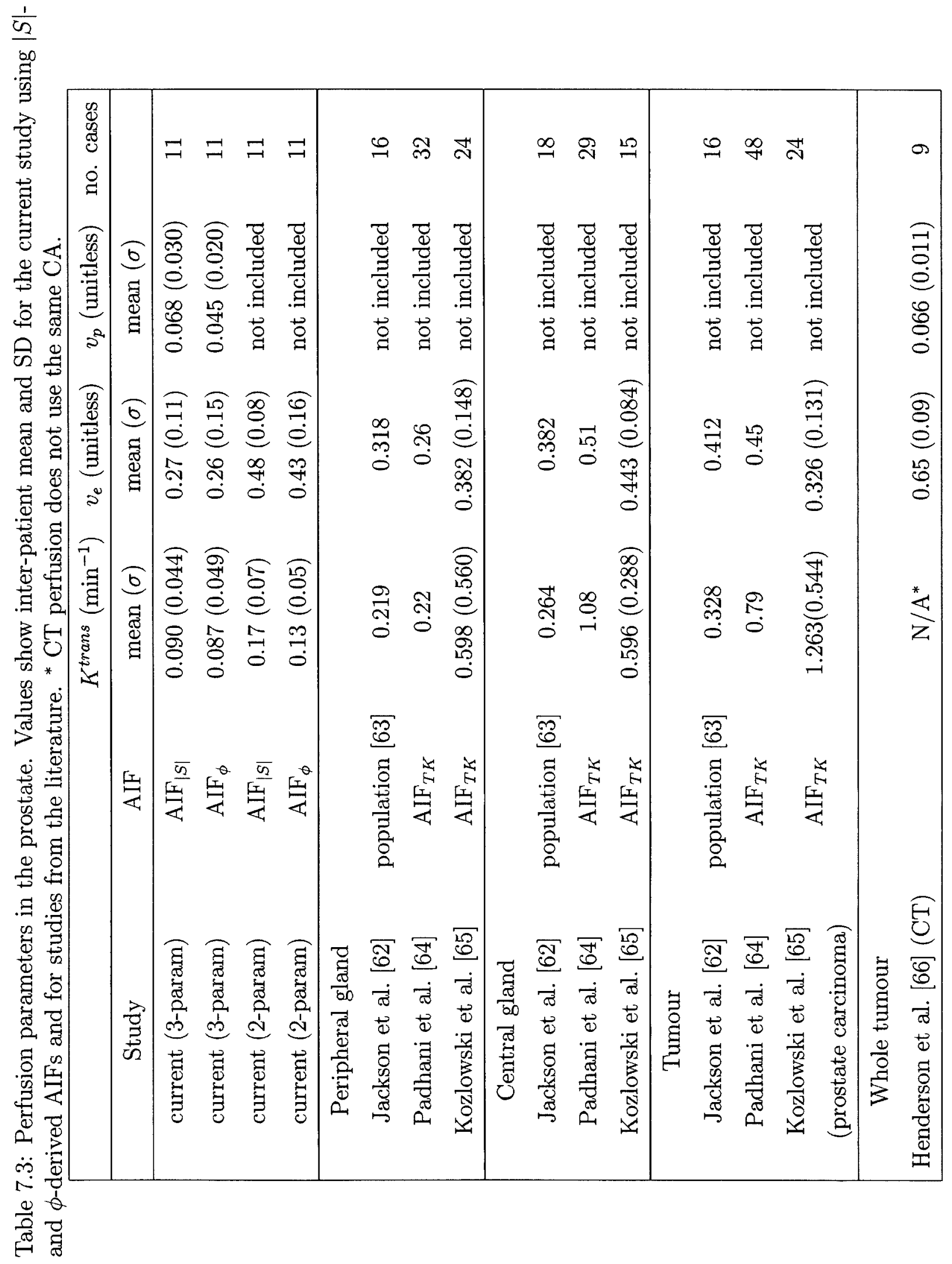


Table 7.4: Maximum of mean tissue $[\mathrm{CA}]$ in $\mathrm{mmol} / \mathrm{kg}$ per $0.1 \mathrm{mmol} / \mathrm{kg}$ injection dose to facilitate comparison with the literature. Values show inter-patient mean and SD for the current study using the $|S|$-derived TRF and for studies from the literature. Note a tissue density of $1 \mathrm{~g} / \mathrm{mL}$ is assumed.

\begin{tabular}{|ccc|}
\hline Study & Location & $\begin{array}{c}{[\mathrm{CA}]_{\max }(\mathrm{mmol} / \mathrm{kg})} \\
\text { mean }(\sigma)\end{array}$ \\
\hline current & whole tumour & $0.42(0.09)$ \\
Padhani et al. [64] & peripheral zone & 0.20 \\
Padhani et al. [64] & central zone & 0.38 \\
Padhani et al. [64] & tumour & 0.33 \\
Kozlowski et al. [65] & peripheral zone & $0.274(0.115)$ \\
Kozlowski et al. [65] & central zone & $0.314(0.069)$ \\
Kozlowski et al. [65] & prostate carcinoma & $0.257(0.099)$ \\
\hline
\end{tabular}

\subsection{Discussion}

DCE-MRI has shown great promise as a key diagnostic tool for pelvic tumours and considerable work has be done on assessing its usefulness. Unfortunately proposed thresholds, which can influence clinical decisions, cannot be applied between institutions if reproducible exam results cannot be achieved. There is a need for a standardized technique which provides perfusion parameters which are as closely linked to the underlying physiology as possible and are reproducible despite a range of imaging protocols and imaging hardware specifications. One component of this challenge is to measure accurate and reproducible AIFs, despite variations in RF homogeneity and image volume size with different imaging protocols. Typical DCE-MRI studies use only $|S|$ in the analysis. The additional $\phi$ information is ignored. There are several reasons to suppose that the phase will provide superior $[\mathrm{CA}]$ information in larger vessels with well known geometries. A goal of this thesis is to show that the AIF obtained from the $\phi$ of the DCE-MRI dataset can provide more accurate AIFs which do not depend on the location of measurement inside the imaging volume. 
In this chapter, two important advancements in this area are demonstrated in a study of prostate tumour patients. These advancements are: 1) $\phi$ was successfully used to measure the AIF in the pelvis, for a DCE-MRI study. This has not been previously reported. 2) The $|S|$-derived AIF varies significantly with the location of measurement within the imaging volume and this has a large effect on the perfusion parameters. The $\phi$-derived AIF does not vary significantly with the location of measurement in the imaging volume and thus provides a more robust method of measuring the AIF.

Overall, this study demonstrated that $\mathrm{AIF}_{\phi}$ can be successfully used to measure perfusion parameters in prostate tumours. The difference between the perfusion results derived using the optimal $\mathrm{AIF}_{\phi}$ versus the optimal $\mathrm{AIF}_{|S|}$ was not as dramatic as seen in the previous, intra-cranial study presented in Chapter 6. This is owing to the different protocol used in this study. The 3D acquisition used here benefits the $|S|$ method, and the shorter $T_{E}$ reduces the $\mathrm{SNR}_{\phi}$. There were however, significant differences in the final AIFs, and some features which favoured the use of phase over magnitude to calculate the AIF.

The perfusion results in the OIM, and the AIFs themselves, show that conventional $\mathrm{AIF}_{|S|}$ techniques are dependent on the location of the ROI used to generate that AIF, whereas phase techniques are independent of the location. The decrease in the calculated $\operatorname{AIF}_{|S|}(z)$ amplitude (both peak and washout) toward superior slices may be attributed to inflow, as has been observed by other investigators [67],[68]. Since blood flows from superior to inferior slices, the more superior slices are expected to be affected the most. Furthermore, the radiofrequency (RF) transmit profile through the 3D imaging slab is not perfect, resulting in reduced flip angles near the superior and inferior edges [34]. These underestimated flip angles lead to underestimated $T_{1}$, with more of an effect at longer $T_{1}$ 's. This also contributes to the decreased measured $\operatorname{AIF}_{|S|}(z)$ amplitude. Any underestimation of the peak height or washout amplitude 
of the AIF will result in an overestimation of the perfusion parameters, as it appears that the extent of the leakage is greater than it is. Thus, for the present study the lowest perfusion parameters are likely to be the most accurate. This "local minimum" can be seen in Fig. 7.3 for the values of $K^{\text {trans }}$ and $v_{e}$ computed using $\operatorname{AIF}_{|S|}(z)$ but it is not observed when $\operatorname{AIF}_{\phi}(z)$ is used to calculate the perfusion parameters.

Using $\phi$ to calculate the AIF can, therefore, offer more flexibility in terms of data acquisition and analysis strategies; allowing for the use of any section of any suitable artery, as long as a reliable artery geometry factor can be determined. For a 3D acquisition, this might allow selection of a vessel closer to the tissue of interest, regardless of the position of that tissue relative to the imaging slab, which would reduce concerns of whether the AIF measured reflected the true input of the tissue.

Even once the $\mathrm{AIF}_{|S|}$ with the highest $[\mathrm{CA}]$ was chosen, the $\mathrm{AIF}_{\phi}$ appears to be more consistent with the literature. The mean peak $[\mathrm{CA}]$ of the optimal $\mathrm{AIF}_{\phi}$ was statistically significantly larger than the mean peak of the optimal $\mathrm{AIF}_{|S|}$ which is in closer agreement with $\mathrm{AIF}_{P}$, suggesting that $\mathrm{AIF}_{\phi}$ is likely an improved AIF compared to $\mathrm{AIF}_{|S|}$. Neither the mean $\mathrm{AIF}_{\phi}$ nor the mean $\mathrm{AIF}_{|S|}$ agreed with $\mathrm{AIF}_{P}$ statistically, both being lower in value. One likely reason for this is the lower time resolution of the current study; $13.5 \mathrm{~s}$ as apposed to $4.97 \mathrm{~s}$ for $\mathrm{AIF}_{P}$ [38]. The lower temporal resolution means that the true maximum [CA] can easily be missed in the examination and thus the peak appears lower. This is an issue irregardless of whether $|S|$ or $\phi$ is used to measure the AIF.

The perfusion parameters, $K^{\text {trans }}$ and $v_{e}$, calculated in the OIM were different when calculated using $\mathrm{AIF}_{\phi}$ versus $\mathrm{AIF}_{|S|}$, with values calculated using $\mathrm{AIF}_{\phi}$ being lower. This lower estimate of the perfusion parameters is consistent with a higher amplitude AIF (i.e. $\mathrm{AIF}_{\phi}>\mathrm{AIF}_{|S|}$ ). The values of $K^{\text {trans }}$ and $v_{e}$ in the OIM for the current study, using the Tofts \& Kermode AIF, were consistent with those found by Padhani et al. who also used the Tofts \& Kermode AIF (t-test, $p>0.49$ ). This 
validates the tissue response and modelling used in this study. The values of $K^{\text {trans }}$ calculated for the current study using the $\mathrm{AIF}_{P}$ were similar to the values of the Yankeelov et al. rat muscle study (t-test, $p>0.18$ ). Whereas the values for $K^{\text {trans }}$ calculated using $\mathrm{AIF}_{\phi}$ and $\mathrm{AIF}_{|S|}$ were similar to the values obtained by Buckley et al. ( $\mathrm{t}$-test, $p>0.50$ ), they differed from the values obtained by Yankeelov et al. (t-test, $p<0.003)$. The values for $v_{e}$ calculated using $\mathrm{AIF}_{\phi}$ and $\mathrm{AIF}_{|S|}$ did not match well with the values from Buckley et al. (t-test, $p=0.06$ and $p=0.007$ respectively), although they were similar to those from Yankeelov et al. (t-test, $p>0.11$ ). Thus, the results are within the range reported by the literature although it is difficult to show whether the perfusion results calculated using $\mathrm{AIF}_{\phi}$ or the optimal $\mathrm{AIF}_{|S|}$ are more correct despite difference in the AIFs themselves.

The study of the prostate perfusion maps did not reveal a significant difference between the measured $K^{t r a n s}$ and $v_{e}$ across the parametric maps, although a difference in $v_{p}$ was observed. The difference in $v_{p}$ may be due to the difference in peak height observed in $\mathrm{AIF}_{\phi}$ or $\mathrm{AIF}_{|S|}$ although the large error bars mean more data is needed to verify the trend. The other parameters, $K^{\text {trans }}$ and $v_{e}$, appear to be unaffected by this difference. This may be due to the larger variability of the pixel by pixel results; additional data may be needed to observe a difference. Also, a low time resolution can contribute to the lack of difference since the peak is not well sampled. Improved time resolution is a constant goal of DCE-MRI and improvements to the hardware which increase time resolution will possibly reveal further differences between the perfusion parameters derived using $\mathrm{AIF}_{\phi}$ and $\mathrm{AIF}_{|S|}$.

There was a significant different in the perfusion parameter measured in the prostate based on whether a two or three parameter model was used. This illustrates the importance of choosing the correct model and the difficulty in comparing results found in the literature if different models are used. This is not a flaw in the proposed technique and exists whether or not $\phi$ is used to measure the AIF. 
The value for $v_{p}$ obtained in this study, using either $\mathrm{AIF}_{\phi}$ or $\mathrm{AIF}_{|S|}$, is within 1 $\mathrm{SD}$ of the value obtained by Henderson et al. using CT [66]; the $v_{e}$ values however do differ. The $K^{\text {trans }}$ values measured in this study are lower than the literature values reported in Table 7.3. These differences can, to a certain extent, be attributed to the different AIFs used. Padhani et al. [64] and Kozlowski et al. [65] both used the population AIF, $\mathrm{AIF}_{T K}$, which is expected to produce higher perfusion values than either $\mathrm{AIF}_{\phi}$ or $\mathrm{AIF}_{|S|}$ as was demonstrated in the study of the OIM, see Table 7.2. Jackson et al. [62] used a population AIF with a higher amplitude [63] and obtained lower values than the other two authors. The range of values reported in the literature clearly demonstrates the importance of establishing a reproducible AIF. Padhani et al. [64] and Kozlowski et al. [65], despite using the same AIF, measured different values of $K^{\text {trans }}$ suggesting that there is either a large patient to patient variability or that there are additional variations in the protocol (i.e. time resolution 10 s versus 22 $\mathrm{s}$, respectively) or analysis technique, which affect the results, or both. Interestingly, the values for $v_{e}$ are much more consistent between the studies, when the same model is applied.

\subsection{Conclusions}

In conclusion, the use of the phase of the MR signal to calculate reliable arterial input functions for dynamic contrast enhanced MRI in extra-cranial tissues is possible. This work has demonstrated the successful development of a protocol which produces similar perfusion parameter values to those achieved using conventional techniques. The $\mathrm{AIF}_{\phi}$ technique has an advantage over $\mathrm{AIF}_{|S|}$ techniques in that it is not dependent on the location of the ROI used to calculate the AIF. Current results suggest that there is a difference in the perfusion parameters calculated using the different techniques, but further validation, perhaps using an additional imaging modality or 
blood sampling, is needed to conclude which is the most accurate. As the addition of the phase component to the DCE-MRI protocol does not require additional scan time, future studies should continue to include MR signal phase; it shows potential benefit and simplifies the AIF selection process. 


\section{Chapter 8}

\section{Conclusion}

\subsection{Overview of Problem}

Tissue perfusion - the delivery of nutrients and removal of waste from a tissue - can provide important diagnostic information in determining the best course of treatment for tumours. Growing tumours recruit new blood vessels to increase their supply of nutrients. These new, rapidly generated blood vessels are often poorly formed, leading to higher vessel permeability in tumours than is seen in healthy tissue. DCE-MRI studies endeavor to assess tissue perfusion by imaging the tissue as an injected contrast agent (CA) passes through the capillaries and diffuses into the surrounding tissue. This can provide useful information about vascular density and vessel permeability. To better understand the underlying physiology, a tracer kinetic model is applied to the data to extract perfusion parameters. To compare these perfusion parameters between patients and institutions, quantitative measurements are required. For quantitative measurement of the perfusion parameters, accurate knowledge of the concentrations of the contrast agent ([CA]) in the tissue (TRF) and a feeding artery (AIF) are required. Current DCE-MRI methods involve using the magnitude of the MR signal, $|S|$, to measure the AIF and TRF, but the high [CA] in the AIF makes an accurate measurement of [CA] using this method difficult. 
Fortunately, the MR signal is a vector and thus has not only a magnitude but also a phase, $\phi$, both of which are affected by the presence of a CA in a quantifiable way. There are several advantages to using $\phi$ for AIF measurements, as were outlined in Chapter 3. The $|S|$ is non-linear with $[\mathrm{CA}]$ and saturates at high [CA]. This means that the peak of the AIF will normally be underestimated when $|S|$ is used to measure the AIF. In contrast, $\phi$ is linear with [CA] and does not saturate at high [CA]. There is some potential for uncertainty in the $\phi$-to- $[\mathrm{CA}]$ conversion if the change in $\phi$ between two data points is more than $2 \pi$. However, this was not a concern in this work, given the relatively short $T_{E}$ 's used in DCE-MRI. Even if the non-linearity of the $|S|$ to [CA] conversion is modelled and the change in $T_{2}^{*}$ is taken into account by using a double echo sequence, difficulties in using the $|S|$ signal remain. The $|S|$ is also sensitive to $B_{1}$ inhomogeneities and blood flow, both of which lead to an underestimation of the AIF. These effects also vary in intensity throughout the image volume, leading to an AIF which is dependent on the location of the ROI used to measure the AIF. For a large 3D imaging volume, these effects can be reduced by careful selection of the AIF measurement location, but this limits the vessels which can be used. It can also be difficult to know if the imaging volume is sufficiently large and where exactly to measure the AIF without first performing careful calibrations. These issues make comparing results between protocols and institutions very difficult when $|S|$ is used to determine the AIF. In contrast, $\phi$ is not affected by $B_{1}$ inhomogeneities or nonturbulent blood flow, and thus $\phi$-derived AIFs can be obtained from anywhere in the imaging volume, or even from a 2D image.

Finally, one must also make a relatively accurate measurement of the pre-contrast $T_{1}$ in the flowing blood when $|S|$ is used to measure the AIF. This requires extra measurements to be performed and can also be very challenging for the reasons stated above. The measurement of $[\mathrm{CA}]$ using $\phi$ however, does not require any pre-contrast information, aside from the pre-bolus baseline dynamic images which are already 
collected for the TRF calculation.

Despite all of these clear advantages for the use of $\phi$ to measure the AIF in DCEMRI, its use is extremely rare and to our knowledge, perfusion parameters from DCEMRI studies using $\phi$-derived $\mathrm{AIFs}, \mathrm{AIF}_{\phi}$, have not been reported in the literature, for human studies. This is possibly because relatively few validation studies have been reported, particularly for short $T_{E}$ 's such as those used in DCE-MRI exams. In addition, a straight-forward method of incorporating the geometry factor has been lacking.

This thesis, motivated by these earlier works, has explored the limits of the $\mathrm{AIF}_{\phi}$ technique in clinically relevant situations and has shown that it is a feasible option which generates more accurate DCE-MRI studies. The work was separated into two components, each of which was presented in two chapters. In Chapters 4 and 5, the implementation of the $\phi$ technique was addressed through phantom studies and simulations based on magnetic susceptibility maps. Chapters 6 and 7 addressed the clinical assessment of the technique in two tumour studies; a brain tumour study and a prostate tumour study.

\subsection{Implementation}

In order to make this technique clinically feasible, some questions about its implementation needed to be addressed. First, the effects of partial volumes and signal leakage were studied using a blood vessel phantom imaged at different resolutions, echo times and geometries. Subsequently, methods to calculate the geometry factor were validated using phantoms and simulations. 


\subsubsection{Phantom Studies}

The initial blood vessel phantom study was conducted to provide validation of the conversion of $\phi$ to [CA]. These experiments involved $\phi$ imaging of plastic tubing which was doped with several $[\mathrm{CA}] \mathrm{s}$, ranging from 0 to $10 \mathrm{mM}$. The straight tubes were imaged at several resolutions, $T_{E}$ 's and angles. For each combination of these three factors, a linear fit of $\Delta \phi$ versus [CA] was calculated and compared to theoretical predictions. The slopes for all resolutions agreed well with theory, with an average percent error of $(3 \pm 5) \%$ for $T_{E}=5.48 \mathrm{~ms}$ and $(-3 \pm 11) \%$ for $T_{E}=2.06 \mathrm{~ms}$. A curved vessel was also investigated under the same range of conditions and the slopes of the linear fits of $\Delta \phi$ versus [CA] were compared to the theoretical value of the slope for an infinite straight cylinder oriented at the same angle as the tangent to the vessel at the point of measurement. The average percent error between measured values and theory, averaged over all FOVs and $|\theta| \leq 25^{\circ}$ for $T_{E}=5.48 \mathrm{~ms}$ was $(-11 \pm 5) \%$ and for $T_{E}=2.06 \mathrm{~ms}$ it was $(-23 \pm 8) \%$. This experiment was probably more susceptible to partial volume and signal leakage effects due to the experimental set up. This agreement suggests that it might be possible to use a "straight vessel approximation" for vessels with a radius of curvature up to that studied here ( $45 \mathrm{~mm}$ for a vessel inner diameter of $9 \mathrm{~mm}$ ).

The effect of geometry (i.e. the orientation of the tubes) on the slopes of these linear fits was very noticeable, and it was concluded that the geometry factor should be accounted for whenever possible. This is not always done with other studies in the literature. When the geometry factor was taken into account, the loss of $\mathrm{SNR}_{\phi}$, as the angle of the vessel with the magnetic field increases was not a factor for the angles studied here $\left(\leq 25^{\circ}\right)$. The effects of partial volumes and signal leakage were observed, although the range of COV values for the results derived at the different FOVs studied was not large, ranging from $(2.0 \pm 0.5) \%$ for the straight vessels at $T_{E}=5.48 \mathrm{~ms}$ to 
$(11 \pm 3) \%$ for the curved tubes at $T_{E}=2.06 \mathrm{~ms}$. These results suggest that, while the effects are definitely seen, they are not large. A straight, thin-walled phantom was also studied; the percent error was greater than for the thick-walled experiment, although the COV was similar. Nonetheless, a larger vessel, where the effects of partial volume averaging are minimal, is preferable and some care should be taken when designing the protocol to ensure that an appropriate vessel is available. The portions of the curved tube at angles greater than $30^{\circ}$ were particularly susceptible to these types of errors.

An expected increase in precision at longer $T_{E}$ 's was also confirmed. While this may be advantageous in some situations, the increase in $T_{E}$ is necessarily accompanied by a decrease in time resolution which is often undesirable. The pros and cons of these two effects must be balanced when designing the protocol.

\subsubsection{Geometry Factor}

The dipole approximation method proposed by Marques and Bowtell [25] was tested to assess whether it could be used as a tool for measuring the geometry factor needed for the $\phi$-to- $[\mathrm{CA}]$ conversion. This method involved generating a 3D map of the vessel from anatomical images and calculating the magnetic field shift caused by the presence of the $\mathrm{CA}$ in that vessel. Accurate results were obtained, provided the background was large relative to the size of the vessel ( $512^{3}$ matrix size was used for most situations), in accordance with the assumptions of the method. This method was also tested using a blood vessel phantom with complex geometry. The $\Delta \phi$ was measured experimentally and compared to the $\Delta \phi$ predicted by the dipole approximation method and was found to be in good agreement; the ratio of the two $\Delta \phi$ had a mean value of 1.00 and a standard deviation of 0.13 . It was observed that portions of the vessel with low $\phi$ contrast-to-noise, as predicted by the dipole approximation method, should be removed from the analysis because they are susceptible to large errors. 
The method was also applied to four clinical cases as a proof of principle. A post-contrast 3D image of the superior sagittal sinus (SSS) was used to generate the susceptibility mask through automatic segmentation. The geometry factor calculated from these segmented vessels was compared to that calculated by applying the "straight vessel approximation" using a $2^{\text {nd }}$ order polynomial fit along the vessel; the results were consistent to at maximum error of $0 \pm 10 \%$. This technique provides a convenient method for measuring the geometry factor which requires minimal user interaction. This method was then used to test the accuracy of the proposed "straight vessel approximation". It was found to be accurate to within $4 \%$ at the centre of a toroidal vessel with a radius of curvature to radius ratio of 8 , for angles ranging from $0^{\circ}-45^{\circ}$. The range of values within the vessel was larger, up to a maximum of $16 \%$ in some cases. In addition, a curved vessel was simulated from the geometry information of the SSS for three patients in the brain tumour study described in Chapter 6. The vessel was constructed by calculating a $2^{\text {nd }}$ order polynomial fit along the SSS and given a circular cross-section with a radius of 16 pixels. The geometry factor was calculated using the dipole approximation method. The results from these simulations agreed with straight vessel approximation values to within $12 \%$.

\subsection{Clinical Implementation}

Once the $\phi$ method had been validated and a method for incorporating the geometry factor had been developed, the method was incorporated into a standard DCE-MRI exam. The motivation to implement this technique clinically is apparent, since $\phi$ has many advantages over $|S|$ in measuring the AIF. In addition, the use of $\phi$ is also desirable because the $\phi$ information can be obtained without lengthening scan times or necessitating extra injections.

Thus, there is no loss of standard protocol data when using the $\phi$ technique; 
the $|S|$ data are also recorded, so standard analysis techniques can still be applied, if desired. This also facilitates the comparison between the two techniques because they are both performed on the same patient at the same time; there are no difficulties or inter-patient variability with image registration or even day-to-day variation. This technique was applied to two studies; a brain study with a $2 \mathrm{D}$ acquisition protocol and a prostate study that used a 3D acquisition.

\subsubsection{Brain Tumour Study}

Signal phase was successfully used to measure the input function needed to calculate perfusion parameters for a 2D DCE-MRI exam. A duel echo spoiled gradient (SPGR) echo sequence was used to reduce $T_{2}^{*}$ effects in the tissue [CA] versus time data (TRF), which is calculated using $|S|$. This sequence also allowed for the comparison of the $\mathrm{VOF}_{\phi} \mathrm{s}$ measured using these two echo times. The study showed that even images taken at a $T_{E}$ of $2.06 \mathrm{~ms}$ can produce accurate $\mathrm{VOF}_{\phi} \mathrm{s}$, although the longer $T_{E}$ of 5.48 ms produced more reliable results.

A 2D sequence was selected to provide the high temporal resolution needed in the brain $(\Delta t=2.16 \mathrm{~s})$. The $2 \mathrm{D}$ acquisition also allowed for the selection of a series of slices through the tumour (and the SSS), as well as an additional slice through the neck. This permitted an AIF from the neck arteries to be measured, meaning that the input and output functions could be compared. It is a common (but not extensively tested) approach to use the VOF measured from the SSS as an input function in $|S|$ based DCE-MRI studies; with the protocol used here it was possible to test this assumption. The $\mathrm{AIF}_{\phi} \mathrm{s}$ and $\mathrm{VOF}_{\phi} \mathrm{s}$ measured in this study were consistent with one another. The perfusion parameters derived from the $\mathrm{AIF}_{\phi}$ and the $\mathrm{VOF}_{\phi}$, which were shifted such that the peaks matched with those of the $\mathrm{AIF}_{\phi}$, produced very similar perfusion parameters. This suggests that a properly time-shifted $\mathrm{VOF}_{\phi}$ can be used in place of $\mathrm{AIF}_{\phi}$ when it is more convenient. This result applies when the 
time resolution is similar or more course than that which was applied here.

An $\operatorname{AIF}_{|S|}$ could not be measured reliably in the neck slice since the $|S|$ from a single 2D slices suffers from dramatic inaccuracies due to blood flow effects. Thus, to compare the $|S|$ and $\phi$ techniques, the $\mathrm{VOF}_{|S|}$ and $\mathrm{VOF}_{\phi}$, measured in the SSS were compared. The $\mathrm{VOF}_{\phi}$ was significantly different from the $\mathrm{VOF}_{|S|}$, and the $\mathrm{VOF}_{\phi} \mathrm{s}$ were more consistent with a population AIF from the literature. The differences in $\mathrm{VOF}_{\phi}$ and $\mathrm{VOF}_{|S|}$ resulted in significantly different perfusion parameters.

\subsubsection{Prostate Tumour Study}

Signal phase was successfully used to measure the AIF needed for perfusion modelling in a 3D DCE-MRI exam. The application of a 3D exam meant that the time resolution was longer than in the previous study $(\Delta t=13.5 \mathrm{~s})$ making it much more difficult to observe differences between the $\phi$ - and $|S|$-derived AIFs due to undersampling of the peak. The high concentration peak is where the biggest difference between $\mathrm{AIF}_{\phi}$ and $\mathrm{AIF}_{|S|}$ is expected and, if the peak is cut off, the difference is harder to observe. In addition, a shorter $T_{E}(2.16 \mathrm{~ms})$ was used to try to improve the time resolution; however, this also reduced the $\mathrm{SNR}_{\phi}$. Despite these additional challenges, the $\mathrm{AIF}_{\phi}$ technique still showed advantages over the $\mathrm{AIF}_{|S|}$ technique. $\mathrm{AIF}_{\phi}$ does not depend on the location used to measure the $\mathrm{AIF}$, whereas $\mathrm{AIF}_{|S|}$ does. These variations in $\mathrm{AIF}_{|S|}$ lead to variations in the corresponding perfusion parameters, whereas those derived from $\mathrm{AIF}_{\phi}$ are relatively constant. This means the ROI used to measure the AIF can be placed anywhere in the imaging volume and it can be much larger when the $\phi$ method is used. Although there are locations in the volume where similar $\mathrm{AIF}_{|S|}$ can be measured, it is difficult to know if the same $\mathrm{AIF}_{|S|}$ would be measured if the imaging protocol was changed (i.e. image size, orientation, location of center of the volume relative to the vessel). This means that it is difficult to compare results between institutions even if the optimal $\mathrm{AIF}_{|S|}$ is chosen in each case. 


\subsection{Summary}

The work presented in this thesis has made important advancements toward the improvement of the accuracy of perfusion parameters derived using MRI. In the first part of the thesis, the implementation and feasibility of the proposed $\phi$ based technique were investigated. The effects of partial volumes and signal leakage were measured for a range of FOVs and $T_{E}$ 's in a blood vessel phantom with straight and curved geometries. The importance of considering the geometry of the vessel was demonstrated and methods for incorporating the geometry factor were validated. These techniques were based on the dipole approximation approach of Marques and Bowtell [25] and an approximate method referred to as the "straight vessel approximation". The validated phase technique was then used in two clinical trials; a 2D brain tumour study and a 3D prostate tumour study. The results of these trials are important because they show that MR signal phase can be used to measure perfusion parameters in a DCE-MRI exam for two very distinct imaging protocols and tumour locations and they revealed several advantages of the $\mathrm{AIF}_{\phi} \mathrm{s}$. The brain study indicated that 1) more reliable input functions can be measured using $\phi$ than with $|S|$ in $2 \mathrm{D}$ acquisitions, 2) $\phi$ can be used to measure the AIF from a single slice, something that is not possible with $|S|$ and 3) $\mathrm{VOF}_{\phi}$ can be used in place of $\mathrm{AIF}_{\phi}$ when this is more convenient. The prostate tumour study showed that the $\mathrm{AIF}_{\phi} \mathrm{s}$ do not vary from slice to slice as is the case with the $\mathrm{AIF}_{|S|} \mathrm{s}$. This simplifies the AIF selection process and allows the AIF to be measured anywhere in the imaging volume. Measuring an accurate AIF using $|S|$ from abdominal or pelvic arteries is very difficult but with the $\phi$ based method a reliable AIF can be obtained.

In conclusion, the use of the MR signal phase for the calculation of the arterial input function in dynamic contrast-enhanced magnetic resonance imaging was shown 
to be feasible and to provide superior results to conventional approaches. The experiments presented in this thesis show clearly that the AIF measured with the signal phase is considerably better than the AIF measured with traditional magnitude based methods. The preliminary clinical studies for brain and prostate tumours indicate that the phase derived AIF will provide more consistent and accurate perfusion parameters. 


\section{List of References}

[1] G. J. Tortora and B. Derrickson, Principles of Anatomy and Physiology. John Wiley \& Sons, Inc., 11 ed., 2006.

[2] R. P. Kennan and H. R. Jäger, Quantitative MRI of the Brain: Measuring Changes Caused by Disease, ch. 11. John Wiley \& Sons Ltd, 2003.

[3] G. J. Parker and A. Padhani, Quantitative MRI of the Brain: Measuring Changes Caused by Disease, ch. 10. John Wiley \& Sons Ltd, 2003.

[4] J. A. Johnson and T. A. Wilson, "A model for capillary exchange," American Journal of Physiology, vol. 210, pp. 1299-1303, 1966.

[5] L. Hermansen and M. Wachtlova, "Capillary density of skeletal muscle in welltrained and untrained men," Journal of Applied Physiology, vol. 30, pp. 860-863, 1971.

[6] P. S. Tofts, G. Brix, D. L. Buckley, J. L. Evelhoch, E. Henderson, M. V. Knopp, H. B. Larsson, T.-Y. Lee, N. A. Mayr, G. J. Parker, R. E. Port, J. Taylor, and R. M. Weisskoff, "Estimating kinetic parameters from dynamic contrastenhanced $T_{1}$-weighted MRI of a diffusable tracer: Standardized quantities and symbols," Journal of Magnetic Resonance Imaging, vol. 10, pp. 223-232, 1999.

[7] M. Lemort, A. C. Canizares-Perez, A. Van der Stappen, and S. Kampouridis, "Progress in magnetic resonance imaging of brain tumours," Current Opinion in Oncology, vol. 19, pp. 616-622, 2007.

[8] S. Cha, "Update on brain tumor imaging: from anatomy to physiology," American Journal of Neuroradiology, vol. 27, pp. 475-487, 2006.

[9] J. Folkman, "Tumor angiogenesis," Cancer, vol. 3, pp. 335-388, 1975.

[10] A. R. Padhani and J. E. Husband, "Dynamic contrast-enhanced MRI studies in oncology with an emphasis on quantification, validation and human studies," Clinical Radiology, vol. 56, pp. 607-620, 2001. 
[11] T. Jeswani and A. R. Padhani, "Imaging tumour angiogenesis," Cancer Imaging, vol. 5, pp. 131-138, 2005.

[12] T. A. M. Egeland, J.-V. Gaustad, I. K. Vestvik, I. C. Benjaminsen, B. Mathiesen, and E. K. Rofstad, "Assessment of fraction of radiobiologically hypoxic cells in human melanoma xenografts by dynamic contrast-enhanced MRI," Magnetic Resonance in Medicine, vol. 55, pp. 874-882, 2006.

[13] A. L. Harris, "Hypoxia-a key regulatory factor in tumour growth," Nature Reviews Cancer, vol. 2, pp. 38-47, 2002.

[14] L. O. Ostergaard, R. M. Weisskoff, D. A. Chesler, C. Gyldensted, and B. R. Rosen, "High resolution measurement of cerebral blood flow using intravascular tracer bolus passages. Part I: Mathematical approach and statistical analysis," Magnetic Resonance in Medicine, vol. 36, pp. 715-725, 1996.

[15] W. W. Orrison, Jr., J. D. Lewine, J. A. Sanders, and M. F. Hartshorne, Functional Brain Imaging. Mosby-Year Book, Inc., 1995.

[16] P. S. Tofts and A. G. Kermode, "Measurement of the blood-brain permeability and leakage space using dynamic MR imaging. 1. fundamental concepts," Magnetic Resonance in Medicine, vol. 17, pp. 357-367, 1991.

[17] C. S. Patlak, R. G. Blasberg, and J. D. Fenstermacher, "Graphical evaluation of blood to brain transfer constants from multiple-time uptake data," Journal of Cerebral Blood Flow and Metabolism, vol. 3, pp. 1-7, 1983.

[18] L. Kershaw and D. L. Buckley, "Precision in measurements of perfusion and microvascular permeability with $T_{1}$-weighted dynamic contrast-enhanced MRI," Magnetic Resonance in Medicine, vol. 56, pp. 86-992, 2006.

[19] K. S. St. Lawrence and T.-Y. Lee, "An adiabatic approximation to the tissue homogeneity model for water exchange in the brain: I. theoretical derivation," $J$ Cerebral Blood Flow and Metabolism, vol. 18, pp. 1365-1377, 1998.

[20] M. E. Haacke, R. W. Brown, M. R. Thompson, and R. Venkatesan, Magnetic resonance imaging: physical principles and sequence design. John Wiley \& Sons, Inc., 1999.

[21] Z.-P. Liang and P. C. Lauterbur, Principles of Magnetic Resonance Imaging. IEEE Press, 2000. 
[22] T. E. Conturo and G. D. Smith, "Signal-to-noise in phase angle reconstruction: Dynamic range extension using phase reference offsets," Magnetic Resonance in Medicine, vol. 15, pp. 420-437, 1990.

[23] A. Cárdenas-Blanco, C. Tejos, P. Irarrazaval, and I. Cameron, "Noise in magnitude magnetic resonance images," Concepts in Magnetic Resonance Part A, vol. 32, pp. 409-416, 2008.

[24] I. S. Gribbestad, K. I. Gjesdal, G. Nilsen, S. Lundgren, M. H. B. Hjelstuen, and A. Jackson, Dynamic Contrast-Enhanced Magnetic Resonance Imaging in Oncology, ch. 1. Springer-Verlag, 2005.

[25] J. Marques and R. Bowtell, "Application of a fourier-based method for rapid calculation of field inhomogeneity due to spatial variation of magnetic susceptibility," Concepts in Magnetic Resonance Part B (Magnetic Resonance Engineering), vol. 25B, pp. 65-78, 2005.

[26] K. Donahue, R. Weisskoff, and D. Burstein, "Water diffusion and exchange as they influence contrast enhancement," Journal of Magnetic Resonance Imaging, vol. 07, pp. 102-110, 1997.

[27] J. M. Wild, J. Woodrow, E. J. R. van Beek, B. Misselwitz, and R. Johnson, "Evaluation of rHA labeled with Gd-DTPA for blood pool imaging and targeted contrast delivery," Contrast Media and Molecular Imaging, vol. 5, pp. 39-43, 2010 .

[28] J. Pintaske, P. Martirosian, H. Graf, G. Erb, K.-P. Lodemann, C. D. Claussen, and F. Schick, "Relaxivity of gadopentetate dimeglumine (Magnevist), gadobutrol (Gadovist), and gadobenate dimeglumine (MultiHance) in human blood plasma at $0.2,1.5$, and 3 Tesla," Investigative Radiology, vol. 41, pp. 213$221,2006$.

[29] L. de Rochefort, T. Nguyen, R. Brown, P. Spincemaille, G. Choi, J. Weinsaft, M. Prince, and Y. Wang, "In vivo quantification of contrast agent concentration using the induced magnetic field for time-resolved arterial input function measurement with MRI," Medical Physics, vol. 35, pp. 5328-5339, 2008.

[30] R. M. Weisskoff and S. Kiihne, "MRI susceptometry: Image-based measurement of absolute susceptibility of MR contrast agents and human blood," Magnetic Resonance in Medicine, vol. 24, pp. 375-383, 1992. 
[31] C. Foottit, G. O. Cron, M. J. Hogan, T. B. Nguyen, and I. Cameron, "Determination of the venous output function from MR signal phase: Feasibility for quantitative DCE-MRI in human brain," Magnetic Resonance in Medicine, vol. 63, pp. $772-781,2010$.

[32] S. C. Deoni, B. K. Rutt, and T. M. Peters, "Rapid combined $T_{1}$ and $T_{2}$ mapping using gradient recalled acquisition in the steady state," Magnetic Resonance in Medicine, vol. 49, pp. 515-526, 2003.

[33] H.-L. M. Cheng and G. A. Wright, "Rapid high-resolution $T_{1}$ mapping by variable flip angles: Accurate and precise measurements in the presence of radiofrequency field inhomogeneity," Magnetic Resonance in Medicine, vol. 55, pp. 566-574, 2006.

[34] G. O. Cron, G. Santyr, and F. Kelcz, "Accurate and rapid quantitative dynamic contrast-enhanced breast MR imaging using spoiled gradient-recalled echos and bookend $T_{1}$ measurements," Magnetic Resonance in Medicine, vol. 42, pp. 746$753,1999$.

[35] H.-L. M. Cheng, " $T_{1}$ measurement of flowing blood and arterial input function determination for quantitative 3D $\mathrm{T}_{1}$-weighted DCE-MRI," Journal of Magnetic Resonance Imaging, vol. 25, pp. 1073-1078, 2007.

[36] H. Weinmann, M. Laniado, and W. Mutzel, "Pharmacokinetics of GdDTPA/Dimeglumine after intravenous injection into healthy volunteers," Physiol. Chem. and Phys. and Medical NMR, vol. 16, 1984.

[37] T. Fritz-Hansen, E. Rostrup, H. B. W. Larsson, L. Sandergaard, P. Ring, and O. Henriksen, "Measurement of the arterial concentration of Gd-DTPA using MRI: A step toward quantitative perfusion imaging," Magnetic Resonance in Medicine, vol. 36, pp. 225-231, 1996.

[38] G. J. Parker, C. Roberts, A. Macdonald, G. A. Buonaccorsi, S. Cheung, D. L. Buckley, A. Jackson, Y. Watson, K. Davies, and G. C. Jayson, "Experimentallyderived functional form for a population-averaged high-temporal-resolution arterial input function for dynamic contrast-enhanced MRI," Magnetic Resonance in Medicine, vol. 56, pp. 993-1000, 2006.

[39] C. de Bazelaire, N. M. Rofsky, G. Duhamel, J. Zhang, M. D. Michaelson, D. George, and D. C. Alsop, "Combined $T_{2}^{*}$ and $T_{1}$ measurements for improved perfusion and permeability studies in high field using dynamic contrast enhancement," European Journal of Radiology, vol. 16, pp. 2083-2091, 2006. 
[40] T. Conturo, E. Akbudak, M. Kotys, M. Chen, S. Chun, R. Hsu, C. Sweeney, and J. Markham, "Arterial input functions for dynamic susceptibility contrast MRI: requirements and signal options," Journal of Magnetic Resonance Imaging, vol. 22 , pp. $697-703,2005$.

[41] G. Cron, J. Wallace, W. Stevens, T. Fortin, B. Pappas, R. Wilkins, F. Kelcz, and G. Santyr, "A comparison of $T_{2}^{*}$-weighted magnitude and phase imaging for measuring the arterial input function in the rat aorta following intravenous injection of gadolinium contrast agent," Magnetic Resonance Imaging, vol. 23, pp. 619-627, 2005.

[42] T. E. Conturo, P. B. Barker, V. P. Mathews, L. H. Monsein, and R. N. Bryan, "MR imaging of cerebral perfusion by phase-angle reconstruction of bolus paramagnetic-induced frequency shifts," Magnetic Resonance in Medicine, vol. 27, pp. 375-390, 1992.

[43] E. Akbudak and T. E. Conturo, "Arterial input functions from MR phase imaging," Magnetic Resonance in Medicine, vol. 36, pp. 809-815, 1996.

[44] M. J. van Osch, E.-J. P. Vonken, C. J. Bakker, and M. A. Viergever, "Correcting partial volume artifacts of the arterial input function in quantitative cerebral perfusion MRI," Magnetic Resonance in Medicine, vol. 45, pp. 477-485, 2001.

[45] M. J. P. van Osch, E. jan P. A. Vonken, M. A. Viergever, J. van der Grond, and C. J. G. Bakker, "Measuring the arterial input function with gradient echo sequences," Magnetic Resonance in Medicine, vol. 49, pp. 1067-1076, 2003.

[46] M. J. van Osch, J. van der Grond, and C. J. Bakker, "Partial volume effects on arterial input functions: shape and amplitude distortions and their correction," Journal of Magnetic Resonance Imaging, vol. 22, pp. 704-709, 2005.

[47] E. Ribot, E. Thiaudire, R. Roulland, P. Brugires, A. Rahmouni, P. Voisin, J. Franconi, and S. Miraux, "Application of MRI phase-difference mapping to assessment of vascular concentrations of BMS agent in mice," Contrast Media Molecular Imaging, vol. 3, pp. 53-60, 2008.

[48] R. Salomir, B. D. de Senneville, and C. T. Moonen, "A fast calculation method for magnetic field inhomogeneity due to an arbitrary distribution of bulk susceptibility," Concepts in Magnetic Resonance Part B (Magnetic Resonance Engineering)), vol. 19, pp. 26-34, 2003. 
[49] E. Akbudak, R. E. Norberg, and T. E. Conturo, "Contrast-agent phase effects: an experimental system for analysis of susceptibility, concentration, and bolus input function kinetics," Magnetic Resonance in Medicine, vol. 38, pp. 990-1002, 1997.

[50] N. Petridou, S. Wharton, A. Lotfipour, P. Gowland, and R. Bowtell, "Investigating the effect of blood susceptibility on phase contrast in the human brain," Neuroimage, vol. 50, pp. 491-498, 2010.

[51] A. R. Padhani, V. S. Khoo, J. Suckling, J. E. Husband, M. O. Leach, and D. P. Dearnaley, "Evaluating the effect of rectal distension and rectal movement on prostate gland position using cine MRI," International journal of radiation oncology, biology, physics, vol. 44, pp. 525-533, 1999.

[52] P. Armitage and G. Berry, Statistical Methods in Medical Research. Blackwell scientific publications, 3 ed., 1994.

[53] K. Murase, "Efficient method for calculating kinetic parameters using t1weighted dynamic contrast-enhanced magnetic resonance imaging," Magnetic Resonance in Medicine, vol. 51, pp. 858-862, 2004.

[54] A. Jackson, G. C. Jayson, K. L. Li, X. P. Zhu, D. R. Checkley, J. J. L. Tessier, and J. C. Waterton, "Reproducibility of quantitative dynamic contrast-enhanced MRI in newly presenting glioma," The British Journal of Radiology, vol. 76, pp. 153-162, 2003.

[55] L. Lüdemann, W. Grieger, R. Wurm, P. Wust, and C. Zimmer, "Quantitative measurement of leakage volume and permeability in gliomas, meningiomas and brain metastases with dynamic contrast-enhanced mri," Magnetic Resonance Imaging, vol. 23, pp. 833-841, 2005.

[56] H. C. Roberts, T. P. L. Roberts, R. C. Brasch, and W. P. Dillon, "Quantitative measurement of microvascular permeability in human brain tumors achieved using dynamic contrast-enhanced $\mathrm{mr}$ imaging: Correlation with histologic grade," AJNR American Journal of Neuroradiology, vol. 21, pp. 891-899, 2000.

[57] R. Jain, S. Ellika, L. Scarpace, L. Schultz, J. Rock, J. Gutierrez, S. Patel, J. Ewing, and T. Mikkelsen, "Quantitative estimation of permeability surfacearea product in astroglial brain tumors using perfusion ct and correlation with histopathologic grade," AJNR Am J Neuroradiol, vol. 29, pp. 694-700, 2008. 
[58] B. Andrews, M. Dujovny, H. Mirchandani, and J. Ausman, "Microsurgical anatomy of the venous drainage into the superior sagittal sinus," Neurosurgery, vol. 24, pp. 514-520, 1989.

[59] D. Buckley, L. Kershaw, and G. Stanisz, "Cellular-interstitial water exchange and its effect on the determination of contrast agent concentration in vivo: dynamic contrast-enhanced MRI of human internal obturator muscle," Magnetic Resonance in Medicine, vol. 60, pp. 1011-1019, 2008.

[60] A. R. Padhani, C. Hayes, S. Landau, and M. O. Leach, "Reproducibility of quantitative dynamic MRI if normal human tissues," NMR in Biomedicine, vol. 15, pp. 143-153, 2002.

[61] T. E. Yankeelov, G. O. Cron, C. L. Addison, J. C. Wallace, R. C. Wilkins, B. A. Pappas, G. E. Santyr, and J. C. Gore, "Comparison of a reference region model with direct measurement of an AIF in the analysis of DCE-MRI data," Magnetic Resonance in Medicine, vol. 57, pp. 353-361, 2007.

[62] A. S. N. Jackson, S. A. Reinsberg, S. A. Sohaib, E. M. Charles-Edwards, S. Jhavar, T. J. Christmas, A. C. Thompson, M. J. Bailey, C. M. Corbishley, C. Fisher, M. O. Leach, and D. P. Dearnaley, "Dynamic contrast-enhanced MRI for prostate cancer localization," The British Journal of Radiology, vol. 82, pp. $148-156,2009$.

[63] S. Walker-Samuel, M. O. Leach, and D. J. Collins, "Evaluation of response to treatment using DCE-MRI: the relationship between initial area under the gadolinium curve (IAUGC) and quantitative pharmacokinetic analysis," Physics in Medicine and Biology, vol. 51, pp. 3593-3602, 2006.

[64] A. R. Padhani, C. J. Gapinski, D. A. Macvicar, G. J. Parker, J. Suckling, P. B. Revell, M. O. Leach, D. P. Dearnaley, and J. E. Husband, "Dynamic contrast enhanced MRI of prostate cancer; correlation with morphology and tumor stage, histological grade and PSA," Clinical Radiology, vol. 55, pp. 99-109, 2000.

[65] P. Kozlowski, S. D. Chang, E. C. Jones, K. W. Berean, H. Chen, and S. L. Goldenberg, "Combined diffusion-weighted and dynamic contrast-enhanced MRI for prostate cancer diagnosiscorrelation with biopsy and histopathology," Journal of Magnetic Resonance Imaging, vol. 24, pp. 108-113, 2006.

[66] E. Henderson, M. F. Milosevic, M. A. Haider, and I. W. T. Yeung, "Functional ct imaging of prostate cancer," Physics in Medicine and Biology, vol. 48, pp. 3085$3100,2003$. 
[67] J. L. Zhang, H. Rusinek, L. Bokacheva, Q. Chen, P. Storey, and V. S. Lee, "Use of cardiac output to improve measurement of input function in quantitative dynamic contrast-enhanced MRI," Journal of Magnetic Resonance Imaging, vol. 30 , pp. $656-665,2009$.

[68] C. Roberts, D. L. Buckley, and G. J. Parker, "To assume or not to assume blood $T_{1}$ for AIF measurement in DCE-MRI?," Proceedings of the 17th meeting of the International Society for Magnetic Resonance in Medicine, p. 2278, 2009.

[69] M. A. Bernstein, M. Grgic, T. J. Brosnan, and N. J. Pelc, "Reconstructions of phase contrast, phased array multicoil data," Magnetic Resonance in Medicine, vol. 32, pp. 330-334, 1994.

[70] P. B. Roemer, W. A. Edelstein, C. Hayes, and O. M. S.P. Souza, "The NMR phased array," Magnetic Resonance in Medicine, vol. 16, pp. 192-225, 1990.

[71] M. A. Bernstein, K. F. King, and X. J. Zhou, Handbook of MRI Pulse Sequences. Elsevier Academic Press, 2004. 


\section{Appendix A}

\section{Multiple Coil Combination}

To provide improved SNR, time resolution and/or spatial resolution multiple receiver coils are often used. These are referred to as phased array coils. The result is multiple sets of images which must be combined it such a way as to achieve the maximum SNR and uniformity. Each coil has a different noise variation determined by the coil construction, the coil loading and the proximity to the patient [69]. Since the coils do not provide equal SNR they are weighted to provide an optimal image. The complex image from each coil is first individually reconstructed and then combined in different ways depending on if the magnitude or phase is desired. Roemer et al. [70] provide a method for combining the magnitude information from a series of $\mathrm{N}$ phased array coils. It involves the sum of squares scaled to account for variations in coil sensitivity,

$$
S=\left\{\sum_{k=0}^{N}\left(\frac{S_{k}}{\sigma_{k}}\right)^{2}\right\}^{1 / 2}
$$

where $S_{k}$ is the magnitude of the signal measured using the $k$ th coil and $\sigma_{k}$ is the noise variation of the $k^{\text {th }}$ coil. Measurements of the noise variation are normally conducted for each individual exam session. To do this, data is collected without gradients or $\mathrm{RF}$ pulses and the complex noise variation is calculated [71, $\mathrm{p}$ 501-502].

In the case where the phase difference is desired the following approach can be 
applied. As discussed in Section 2.6.2 the absolute phase value is not of interest, it is the phase difference which provides useful information in the dynamic series. The phase offset of images measured by different coils may not be the same and for this reason it is preferrable to calculate the phase difference before combining the phases from the different coils. Define the phase difference for the $k^{\text {th }}$ coil as

$$
\Delta \phi_{k}=\phi_{B, k}-\phi_{A, k}
$$

where $\phi_{A, k}$ and $\phi_{B, k}$ are the phase at two different time points for the same voxel location. The derivation presented here follows a derivation presented in [69] except they assume the magnitude remains the same for all measurements (i. e. $S_{A}=S_{B}$ ) and here that assumption is not made. It is shown that the final result is approximately the same as found in [69]. Begin by considering the minimization of the variance of a linear combination of the $\Delta \phi_{k}$ 's by weighting each element of the sum by its variance.

$$
\Delta \phi=\frac{1}{C} \sum_{k=0}^{N} \frac{\Delta \phi_{k}}{\sigma_{\Delta \phi_{k}}^{2}},
$$

where

$$
C=\sum_{k=0}^{N} \frac{1}{\sigma_{\Delta \phi_{k}}^{2}} .
$$

Summing the phase differences directly can lead to errors when the phase difference is close to $\pi$. Consider two phase differences which are close to $\pi$ but are affected by a small amount of noise, $\epsilon$. Let these phase differences be $\Delta \phi_{1}=\pi+\epsilon$ and $\Delta \phi_{2}=-\pi-\epsilon$. In this case if the phase differences are summed directly the total phase difference would be zero. If instead exponentials are used, the correct value of $\pi$ is obtained. For this reason it is preferable to perform the summation in terms of exponentials to avoid problems with discontinuities. Thus, the following expression 
is used instead of Eq. A.4 [69]:

$$
\exp (i \Delta \phi)=\frac{1}{K} \sum_{k=0}^{N} \frac{\exp \left(i \Delta \phi_{k}\right)}{\sigma_{\exp \left(i \Delta \phi_{k}\right)}^{2}}
$$

where

$$
K=\sum_{k=0}^{N} \frac{1}{\sigma_{\exp \left(i \Delta \phi_{k}\right)}^{2}}
$$

$\sigma_{\exp \left(i \Delta \phi_{k}\right)}^{2}$ can be written in terms of $\sigma_{k}^{2}$ by applying propagation of errors:

$$
\sigma_{\exp \left(i \Delta \phi_{k}\right)}^{2}=\frac{\partial \exp (i \Delta \phi)}{\partial \Delta \phi_{k}} \frac{\partial \exp (-i \Delta \phi)}{\partial \Delta \phi_{k}}=\sigma_{\Delta \phi_{k}}^{2}
$$

and

$$
\begin{aligned}
\sigma_{\Delta \phi_{k}}^{2} & =\sigma_{\phi_{A, k}}^{2}+\sigma_{\phi_{B, k}}^{2} \\
& =\frac{\sigma_{A, k}^{2}}{S_{A, k}^{2}}+\frac{\sigma_{B, k}^{2}}{S_{B, k}^{2}}
\end{aligned}
$$

where the second line is found by applying Eq. 2.72. It is reasonable to assume that the noise from a given coil will not change in time. Thus $\sigma_{A, k}^{2}=\sigma_{B, k}^{2}=\sigma_{k}^{2}$.

$$
\sigma_{\Delta \phi_{k}}^{2}=\sigma_{k}^{2}\left(\frac{S_{A, k}^{2}+S_{B, k}^{2}}{S_{A, k}^{2} S_{B, k}^{2}}\right)
$$

Substitution Eq. A.10 into Eq. A.5 gives,

$$
\exp (i \Delta \phi)=\frac{1}{K} \sum_{k=0}^{N} \frac{Z_{B, k} Z_{A, k}^{*}}{\sigma_{k}^{2}}\left(\frac{S_{A, k} S_{B, k}}{S_{A, k}^{2}+S_{B, k}^{2}}\right)
$$

where $Z_{B, k}=S_{B, k} \exp \left(i \phi_{B, k}\right)$, the complex signal from the $k^{\text {th }}$ coil for measurement $B$ and $Z_{A, k}^{*}$ is the complex conjugate of $Z_{A, k}$. The magnitude for the $i^{\text {th }}$ pixel measured using the $\mathrm{k}^{\text {th }}$ coil is equal to the noiseless signal plus noise, $S_{i, k}=A_{i, k}+e_{i, k}$. The 
noiseless signal is equal to the underlying signal plus an amplification term which is different for each coil, $A_{i, k}=G_{k} a_{i}$. If the error term is small compared to $A_{i, k}$ then

$$
\left(\frac{S_{A, k} S_{B, k}}{S_{A, k}^{2}+S_{B, k}^{2}}\right) \approx\left(\frac{a_{A} a_{B}}{a_{A}^{2}+a_{B}^{2}}\right)
$$

Thus, the ratio is approximately the same for each coil and can be factored out as a constant. This will not affect the result once the argument of the expression is calculated so it can be entirely ignored. This leads to the following expression,

$$
\Delta \phi=\arg \left\{\sum_{k=0}^{N} \frac{Z_{B, k} Z_{A, k}^{*}}{\sigma_{k}^{2}}\right\}
$$

where $\arg Z=\arctan \frac{i m(Z)}{r e(Z)}$. 


\section{Appendix B}

\section{Code for Marques and Bowtell method}

This program is based on the method described by Marques and Bowtell [25]. It is written in IDL versions 6.2-7.0 (ITT Visual Information Solutions). Refer to Section 2.7.3 and Section 5.2.1 for futher details on theory and implimentation. Note that in IDL a semicolon (;) indicates a commented line.

\section{B.1 Main Body of the Code}

PRO BowtellSimulation

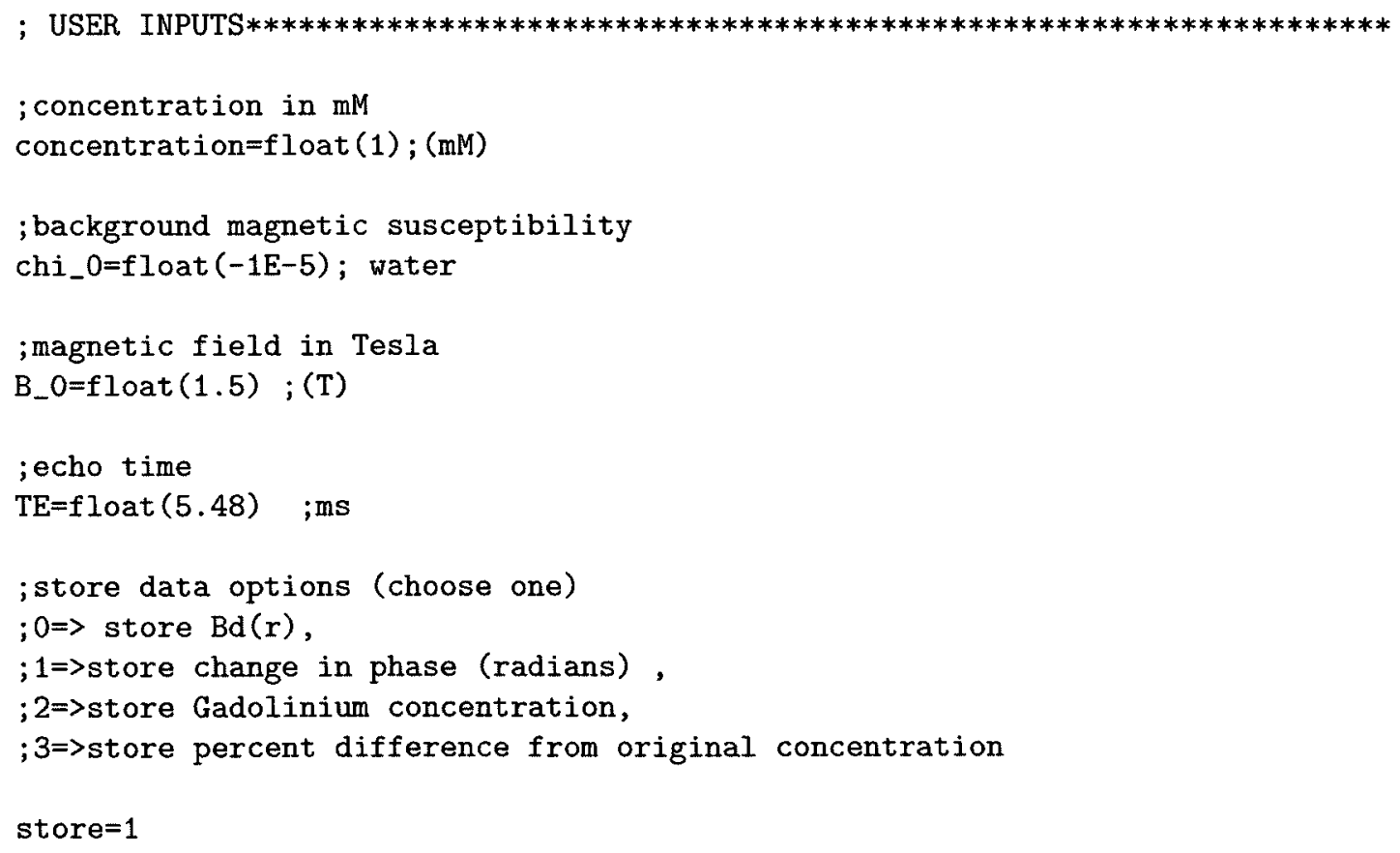




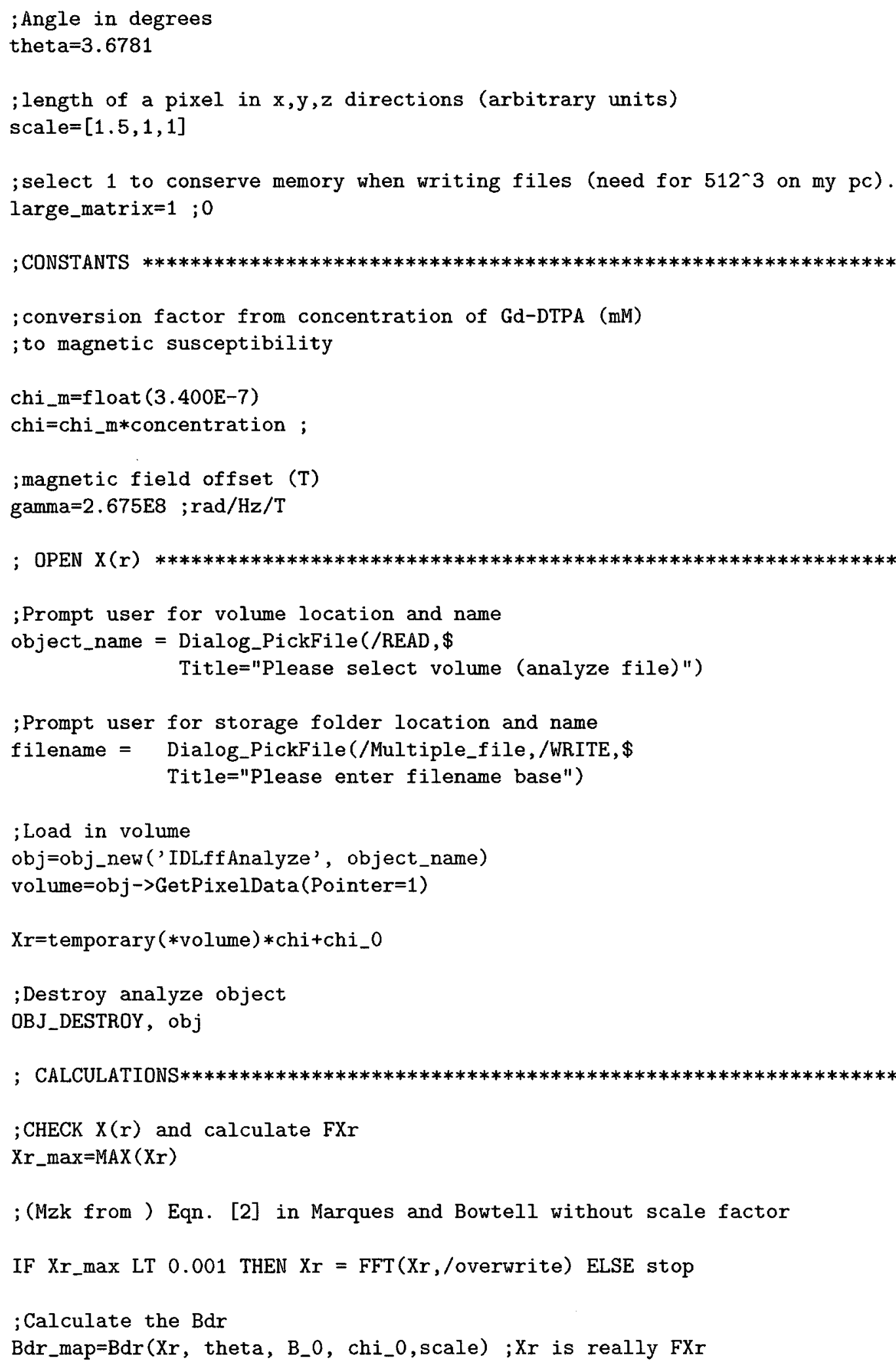




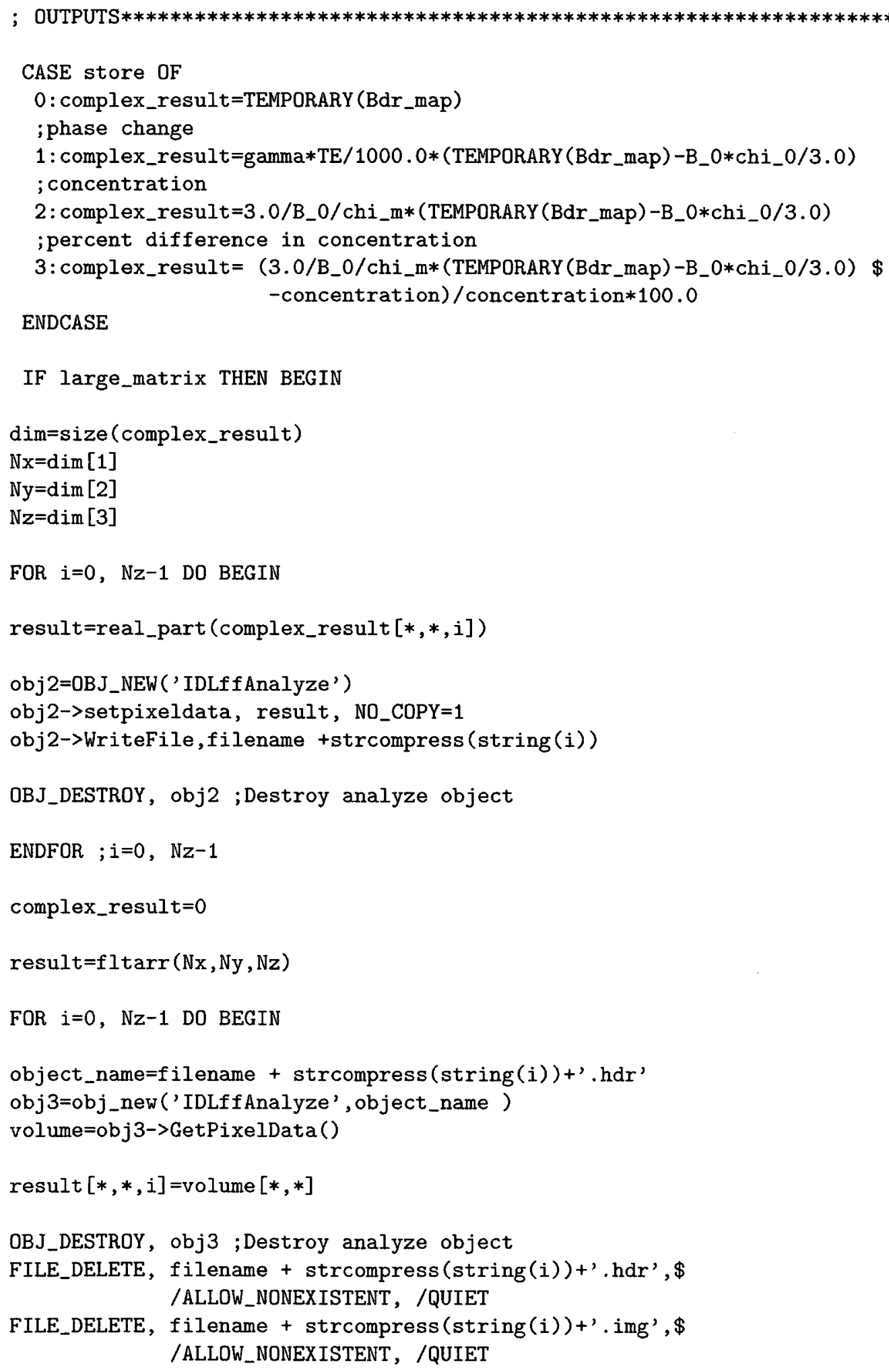




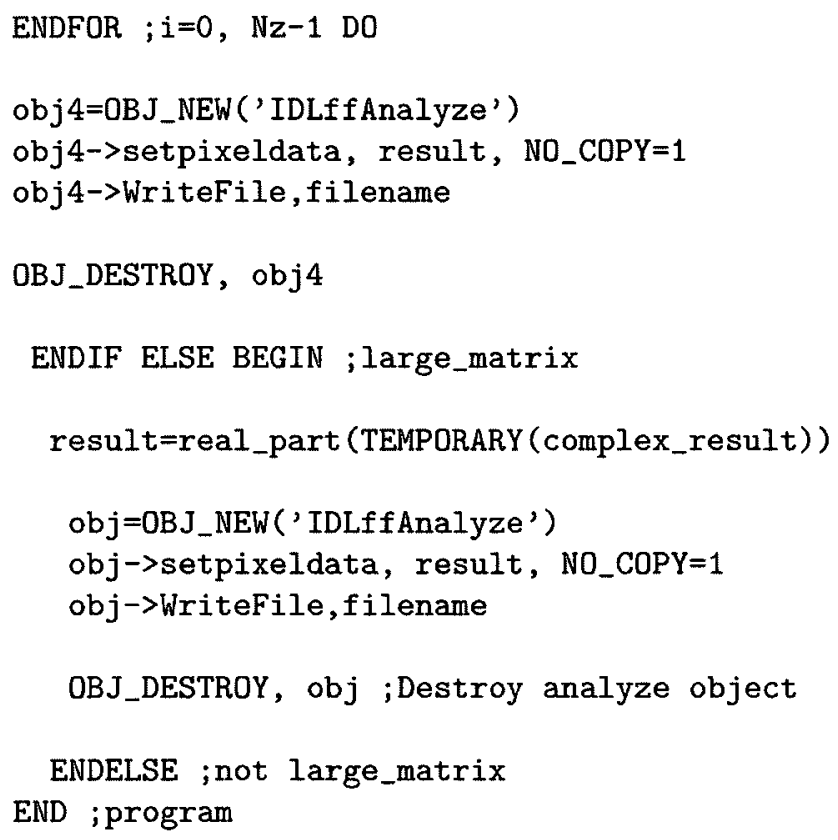

\section{B.2 The Function $B d r$}

This function calculates $\mathbf{B}_{d}(\mathbf{r})$ from a Fourier transform of $\chi(\mathbf{r})$.

FUNCTION Bdr, FXr, theta, BO, Xe,scale

; FXr is the fourier transform of the magnetic susceptibility, $\mathrm{Xr}$ ; theta is the angle in degrees between the $z$ axis and the external ; B field about the $y$ axis.

;BO is the external magnetic field strength in Tesla

; Xe is the magnetic susceptibility of the background material

; Scale is a 3 element array with the scale of the pixel in each dimension.

$\mathrm{PI}=! \mathrm{PI}$

cos_theta $=\operatorname{COS}($ double $($ theta $/ 180.0) * P I)$

sin_theta $=\mathrm{SIN}($ double $($ theta/180.0)*PI)

$\operatorname{dim}=\operatorname{size}(\mathrm{Fxr})$

$\mathrm{Nx}=\operatorname{dim}[1]$

$\mathrm{Ny}=\operatorname{dim}[2]$

$\mathrm{Nz}=\operatorname{dim}[3]$

$\mathrm{NOOx}=\operatorname{ceil}((\mathrm{float}(\mathrm{Nx})-1.0) / 2.0) ;$ NOOx $=$ index of center

$\mathrm{NOOy}=\operatorname{ceil}(($ float $(\mathrm{Ny})-1.0) / 2.0) ; \mathrm{NOOy}=$ index of center

$\mathrm{NOOz}=\operatorname{ceil}((\mathrm{float}(\mathrm{Nz})-1.0) / 2.0) ; \mathrm{NOOz}=$ index of center

$\mathrm{Bdk}=-(\mathrm{BO} / 3.0) *$ TEMPORARY $(\mathrm{FXr})$

for $\mathrm{kx}=0, \mathrm{Nx}-1$ do begin

for $\mathrm{ky}=0, \mathrm{Ny}-1$ do begin

for $\mathrm{kz}=0, \mathrm{Nz}-1$ do begin 


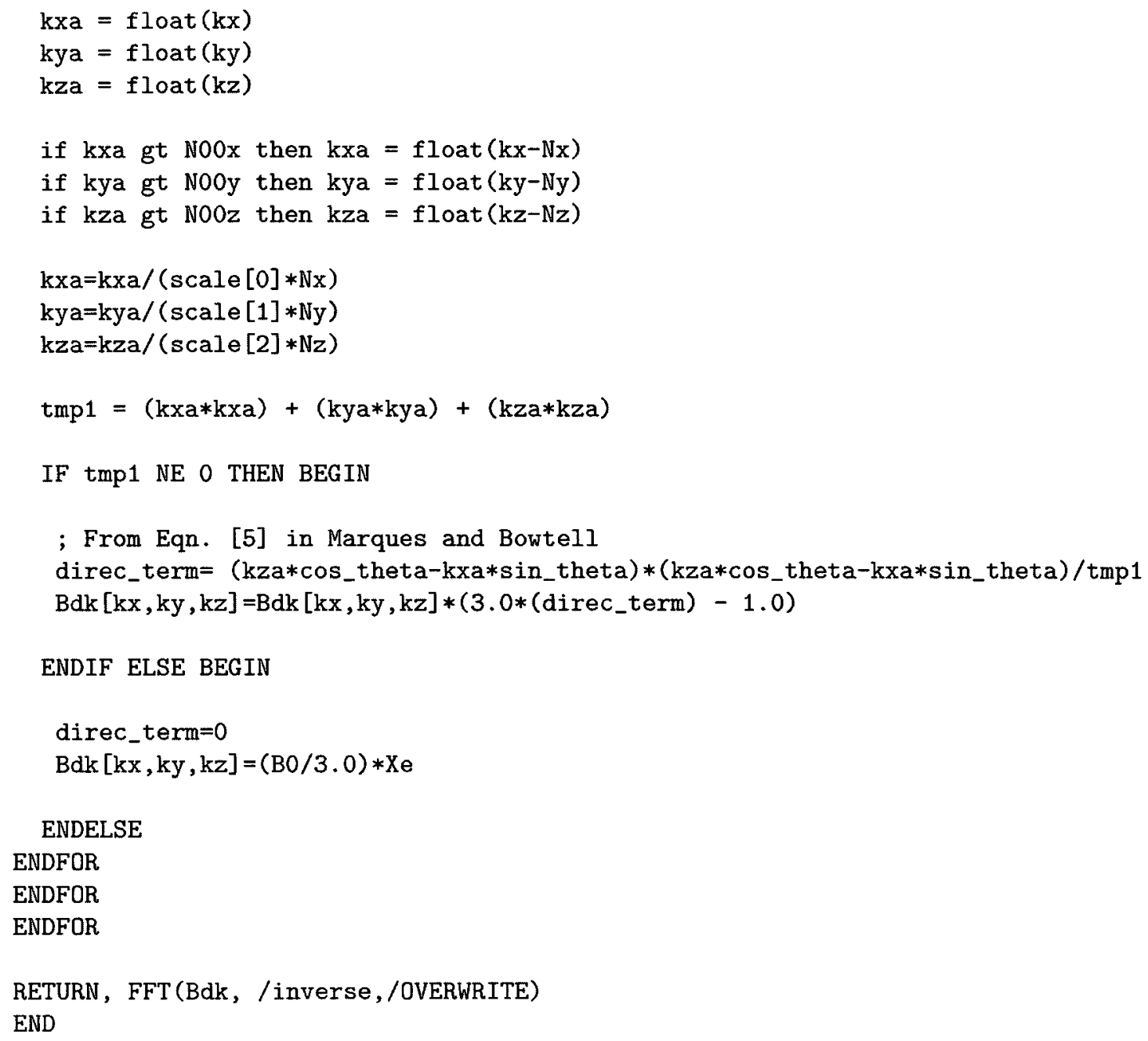

